\title{
Childhood obesity prevention : rationale, implementation and effectiveness of the Lifestyle Triple $\mathbf{P}$ intervention
}

Citation for published version (APA):

Gerards, S. M. P. L. (2014). Childhood obesity prevention : rationale, implementation and effectiveness of the Lifestyle Triple $P$ intervention. [Doctoral Thesis, Maastricht University]. Datawyse / Universitaire Pers Maastricht. https://doi.org/10.26481/dis.20140212sg

Document status and date:

Published: 01/01/2014

DOI:

10.26481/dis.20140212sg

Document Version:

Publisher's PDF, also known as Version of record

\section{Please check the document version of this publication:}

- A submitted manuscript is the version of the article upon submission and before peer-review. There can be important differences between the submitted version and the official published version of record.

People interested in the research are advised to contact the author for the final version of the publication, or visit the DOI to the publisher's website.

- The final author version and the galley proof are versions of the publication after peer review.

- The final published version features the final layout of the paper including the volume, issue and page numbers.

Link to publication

\footnotetext{
General rights rights.

- You may freely distribute the URL identifying the publication in the public portal. please follow below link for the End User Agreement:

www.umlib.nl/taverne-license

Take down policy

If you believe that this document breaches copyright please contact us at:

repository@maastrichtuniversity.nl

providing details and we will investigate your claim.
}

Copyright and moral rights for the publications made accessible in the public portal are retained by the authors and/or other copyright owners and it is a condition of accessing publications that users recognise and abide by the legal requirements associated with these

- Users may download and print one copy of any publication from the public portal for the purpose of private study or research.

- You may not further distribute the material or use it for any profit-making activity or commercial gain

If the publication is distributed under the terms of Article $25 \mathrm{fa}$ of the Dutch Copyright Act, indicated by the "Taverne" license above, 


\section{Childhood obesity prevention}

Rationale, implementation and effectiveness

of the Lifestyle Triple P intervention 


\section{Colophon}

Graphic design: Datawyse / Universitaire Pers Maastricht Production: Datawyse / Universitaire Pers Maastricht

(C) Sanne Gerards, Maastricht, 2014

ISBN 9789461592996

The research presented in this this was performed within the NUTRIM School for Nutrition, Toxicology and Metabolism, Maastricht University. The work was supported by the Netherlands Organization for Health Research and Development (ZonMW), grant number 120520009. 


\title{
Childhood obesity prevention Rationale, implementation and effectiveness of the Lifestyle Triple P intervention
}

\author{
PROEFSCHRIFT \\ ter verkrijging van de graad van doctor aan de Universiteit Maastricht, \\ op gezag van de Rector Magnificus, Prof. dr. L.L.G. Soete, \\ volgens het besluit van het College van Decanen, in het openbaar te verdedigen \\ op woensdag 12 februari 2014 om 14.00 uur \\ door \\ Sandrine Maria Paulien Leonie Gerards
}

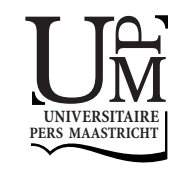




\section{Promotores}

Prof. dr. N.K. De Vries

Prof. dr. P.C. Dagnelie

Prof. dr. S.P.J. Kremers

\section{Beoordelingscommissie}

Prof. dr. G. Kok (voorzitter)

Prof. dr. A. Jansen

Prof. dr. N. Lien (University of Oslo)

Dr. A.C.E. Vreugdenhil

Dr. I.M. de Graaf (Trimbos Instituut) 


\section{Contents}

$\begin{array}{lll}\text { Chapter } 1 & \text { General introduction } & 7\end{array}$

Chapter 2 General parenting, childhood overweight and obesity-inducing behaviors: a review

Chapter 3 Interventions addressing general parenting to prevent or treat childhood obesity

Chapter 4 Parental perception of their child's weight status and its association with longitudinal BMI development in childhood: the KOALA Birth Cohort Study

Chapter 5 Parental self-efficacy in childhood overweight: validation of the Lifestyle Behavior Checklist in the Netherlands

Chapter 6 Barriers to successful recruitment of parents of overweight children for an obesity prevention intervention: a qualitative study among youth health care professionals

Chapter 7 Lifestyle Triple P: a parenting intervention for childhood obesity Study protocol

Chapter 8 The effectiveness of Lifestyle Triple P in the Netherlands: a Randomized Controlled Trial

Chapter 9 General discussion

References 

CHAPTER 1

General introduction

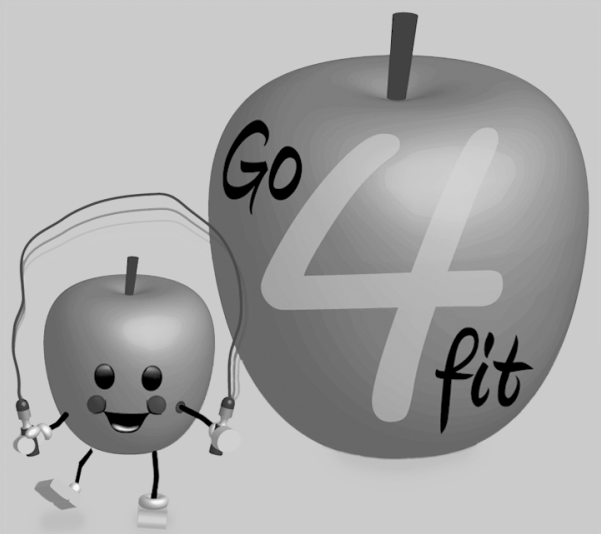


The prevalence of childhood overweight and obesity is increasing dramatically worldwide (De Onis, Blössner, \& Borghi, 2010; WHO, 2013). In the Netherlands, $12.8 \%$ of boys and $14.8 \%$ of girls aged 2 to 21 years were classified as overweight and $1.8 \%$ of the boys and $2.2 \%$ of the girls were identified as obese in 2009. These numbers represent a two- to three-fold increase of overweight prevalence rates compared to 1980 (Schonbeck et al., 2011).

Overweight and obesity are characterized by 'abnormal or excessive fat accumulation that presents a risk for health' (WHO, 2013). Children who are overweight or obese are at increased risk of becoming our obese adult population (Singh, Mulder, Twisk, Van Mechelen, \& Chinapaw, 2008). Furthermore, overweight children are at increased risk of developing a range of short- and long-term negative consequences (Daniels, 2009), for example cardiovascular diseases, type 2 diabetes, sleep disorders, and asthma. In addition to these physical consequences, overweight children often suffer from psychosocial consequences like low self-esteem, negative body image, and depression (Must \& Strauss, 1999).

Overweight and obesity are caused by a chronically disturbed energy balance, in which the energy intake exceeds the energy expenditure. So-called 'energy balancerelated behaviors' (EBRBs) (Kremers, Visscher, Seidell, Van Mechelen, \& Brug, 2005) include a diet high in fat and low in fiber, frequent snacking, frequent consumption of soft drinks, a high level of sedentary behavior and low levels of physical activity (WHO, 2003). Obesity in young children is largely explained by a low total physical activity level, and increased time spent on television viewing (Rennie, Johnson, \& Jebb, 2005; Te Velde et al., 2012). Diet-related risk factors include high-energy diets, high consumption of sugar-sweetened beverages, large portion sizes and eating patterns (Perez-Escamilla et al., 2012; Rennie et al., 2005). These unhealthy dietary and physical activity behaviors are likely to track into adulthood (Kelder, Perry, Klepp, \& Lytle, 1994). Interactions between individual and environmental factors causing these EBRBs complicate the determinants of the development of childhood obesity (Kremers et al., 2006).

It is important to start prevention of childhood obesity and overweight at a young age. Overweight at a young age is a predictor of future weight gain (Singh et al., 2008), making it relatively easy to identify risk groups for excessive weight at this age. Another reason for prevention efforts among children is that children have a relatively short history of unhealthy habits, making it easier to change these behaviors compared to adult populations. Furthermore, as their height increases, children can return to a healthy BMI without actively losing weight.

Childhood obesity interventions have traditionally mainly aimed at changing children's individual physical activity and diet. However, there has recently been a trend towards including environmental factors in the intervention. Interventions for children 
are implemented in different setting: the community, the school, and the family/home environment. It is especially among younger children that the family/home environment is very important in shaping their health-related behaviors, and involving parents had been found to be an essential component of interventions promoting healthy weight and lifestyle behaviors among children (Golley, Hendrie, Slater, \& Corsini, 2011; Skouteris et al., 2011).

\section{Youth Health Care}

The Dutch youth health care ( $\mathrm{YHC}$ ) system provides preventive health care to children aged 0-19 years. All children living in the Netherlands are systematically monitored in terms of their physical, psychological, social, and cognitive health. YHC professionals counsel parents and children in these respects and identify possible health problems such as depression, aggression and overweight. If necessary, the YHC professionals refer children to other health care facilities (Verloove-Vanhorick et al., 2002).

Since there are no evidence-based interventions for the prevention of childhood obesity in the Netherlands (Bulk-Bunschoten, Renders, Leerdam, \& Hirasing, 2004), the YHC has been implementing an overweight prevention protocol ('the Be Active Eat Right study'), which is based on promising existing interventions (Veldhuis et al., 2009b). The protocol has four aims: reducing the consumption of sugar-sweetened beverages, increasing physical activity levels and outdoor playing, reducing TV viewing and computer games, and having a healthy breakfast. An evaluation study towards the effects of the bridging protocol showed no overall difference between the intervention and control condition with regard to BMI and waist circumference (Van Grieken et al., 2013). Solely mildly overweight children (BMI 17.25 - 17.50) showed a smaller increase in $\mathrm{BMI}$ at 2 years compared to the control condition at follow-up.

In view of the current lack of evidence-based interventions, it is important that such interventions are developed and implemented. More and more research has underlined the importance of involving parents in the prevention of childhood obesity (Golley et al., 2011; Niemeier, Hektner, \& Enger, 2012). The present thesis focuses on the evaluation of a childhood obesity intervention aimed at parents, called Lifestyle Triple P. Lifestyle Triple P is based on the Triple P - Positive Parenting Program. The general Triple P program, and Lifestyle Triple P in particular, are described below.

\section{Triple P - Positive Parenting Program}

The Triple P-Positive Parenting Program (Triple P) is a multi-level, preventive parenting and family support strategy developed at the University of Queensland in Brisbane, 
Australia, and has evolved through more than 30 years of research (Sanders, MarkieDadds, \& Turner, 2003). Triple P aims to enhance parental knowledge, skills, and confidence in order to prevent behavioral, emotional, and developmental problems in their children (Sanders et al., 2003).

\section{Aims of Triple $\mathrm{P}$}

1. Enhancing the knowledge, skills, confidence, self-sufficiency, and resourcefulness of parents

2. Promoting a nurturing, safe, engaging, non-violent, and low-conflict environment

3. Promoting children's social, emotional, language, intellectual, and behavioral competencies through positive parenting practices

Source: Sanders et al., 2003

The program content of Triple P draws on various theoretical concepts (Sanders et al., 2003). First of all, Triple $P$ is a behavioral family intervention based on social-learning models of parent-child interaction (Patterson, 1982). These models highlight the reciprocal and bi-directional nature of parent-child interaction, so the program guides parents in developing positive child management skills. Secondly, research into child and family behavior therapy and applied behavior change strategies has identified many useful behavior change techniques (Sanders, 1992, 1996). Triple P is also based on developmental research into parenting in everyday contexts. Children are at reduced risk of developing behavioral and emotional problems when their parents use naturally occurring everyday interactions in an emotionally supportive context to teach their children language skills, social skills, developmental competencies and problem-solving skills (Hart \& Risley, 1995). Thirdly, the intervention is based on social information processing models which emphasize the importance of parental cognitions influencing their self-efficacy, decision making and behavioral intentions (Bandura, 1977, 1995). Fourthly, Triple $P$ also integrates findings from the field of psychopathology that have linked specific risk and protective factors with adverse developmental outcomes in children (Emery, 1982; Grych \& Fincham, 1990; Rutter, 1985). These risk factors include coercive parent-child interaction, parental depression, and marital conflict. The fifth and final theoretical underpinning on which Triple $P$ is based is a population health perspective on family interventions, which recognizes the role of the broader ecological context in human development (Biglan, 1995).

The hypothesis behind the program is that effective parenting reduces the risk of children developing serious mental health problems. When parents are competent and 
have a high degree of self-efficacy toward raising their children, and use adaptive ways of responding to dysfunctional behavior, children are less likely to develop behavioral and emotional disorders. Furthermore, children will be at reduced risk of developing significant problems when parents communicate well and use positive parenting strategies. However, parenting does not occur in isolation, but is part of a wider social context. Positive parenting is thus more easily accomplished in a supportive community which acknowledges the importance of positive parenting methods.

Triple $\mathrm{P}$ adopts a public health model to parenting support, which emphasizes the need to target parents at a population level rather than aim at individual improvement (Prinz \& Sanders, 2007). Another key component of the Triple P intervention is the principle of self-regulation. Self-regulation is a process in which individuals learn skills they need in order to change their own behavior and become independent problemsolvers (Sanders \& Mazzucchelli, 2013). Triple P aims to increase parental selfregulation, in order to eventually improve their children's self-regulatory capacity. Five key elements are important in the operationalization of self-regulation: selfmanagement tools (to change their own parenting practices), parental self-efficacy (their sense of confidence about solving personal problems), personal agency (attributing change to their own or their child's efforts), self-sufficiency (considering parenting decisions as their own responsibility) and problem-solving (becoming troubleshooters and active problem-solvers).

Triple P consists of five levels of interventions on a continuum of increasing intensity for parents of children and adolescents (Sanders, 2012). The rationale of this multilevel strategy is that children have different levels of dysfunctional and problem behaviors and that parents have different needs and preferences regarding the delivery mode and intensity of the intervention they require. Together with the multilevel strategy, minimal sufficiency serves as a guiding principle to serve the needs of parents.

Level 1, universal Triple $\mathrm{P}$, comprises the implementation of media and informational strategies relating to positive parenting (Sanders, 2012). These strategies are implemented in order to de-stigmatize parenting and family support, to make effective parenting strategies accessible to all parents and to facilitate help-seeking by parents.

Selected Triple P (level 2) is implemented to normalize parenting interventions, and can be delivered in two forms: a brief consultation with individual parents, or parenting seminars with a large group of parents. The brief consultation intervention consists of one or two 20-minute consultations with parents in order to discuss relatively minor problem behaviors. Parenting seminars include three 90-minute sessions for a large group of parents addressing three topics: the power of positive parenting, raising confident and competent children, and raising resilient children. 
Primary care Triple P (level 3) comprises the delivery of advice and information to parents, supplemented with active skills training. It consists of four brief consultations with parents, in which uncomplicated child problem behaviors are discussed.

Standard Triple P (level 4) is suitable for parents who are struggling with parenting challenges and children who have detectable problems but do not yet meet diagnostic criteria. Two delivery settings can be used: an individual or a group setting. The level 4 intervention consists of providing information, active skills training and support, and teaching parents to apply skills to a broad range of behaviors.

Finally, enhanced Triple $P$ (level 5) is appropriate for families with additional risk factors that may need to be addressed through the intervention. These additional issues include aspects like partner communication, mood management, and childhood obesity.

The evidence base for Triple $\mathrm{P}$ has been summarized in seven meta-analyses conducted by different researchers (De Graaf, Speetjens, Smit, De Wolff, \& Tavecchio, 2008a; De Graaf, Speetjens, Smit, De Wolff, \& Tavecchio, 2008b; Fletcher, Freeman, \& Matthey, 2011; Nowak \& Heinrichs, 2008; Tellegen \& Sanders, 2013; Thomas \& Zimmer-Gembeck, 2007; Wilson et al., 2012). In all of these meta-analyses, the authors concluded that Triple P produces positive effects on children's behavior and parenting practices, with effect sizes ranging from small to large positive effects, with a wide range.

\section{Lifestyle Triple $\mathbf{P}$}

Lifestyle Triple $P$ is a 14-week intervention in which parents are introduced to a range of nutrition, physical activity, and positive parenting strategies. A detailed description of the content of the intervention is provided in chapter 6 of this thesis. A randomized controlled trial on the efficacy of the intervention, conducted by the University of Queensland in Australia, showed that after the intervention, the children had a significantly decreased body size and less weight-related problem behavior, while the parents had increased their confidence as regards managing weight-related problem behavior, and had reduced their ineffective parenting (West, Sanders, Cleghorn, \& Davies, 2010b). So far, however, the evidence base regarding Lifestyle Triple P is limited to this one single study by the University of Queensland (West et al., 2010b). We translated and adapted the intervention to the Dutch context to investigate whether Lifestyle Triple P would be effective in the Netherlands as well. This was the first study outside Australia to test the effectiveness of the Lifestyle Triple P intervention. 


\section{Outline of the thesis}

The present thesis describes the rationale, implementation and evaluation of Lifestyle Triple $P$ in the Netherlands. The second chapter describes the findings of a systematic review on the influence of general parenting on children's weight status and related dietary and activity behaviors. Chapter 3 reports on a systematic review of intervention studies addressing general parenting to prevent or treat childhood obesity. The studies presented in chapters 4 and 5 took place among parents of both overweight and normal weight children. In the study described in chapter 4, we investigated whether parents are able to accurately perceive their child's weight status, and whether accurate parental perception of their children's weight status predicts future weight development. Chapter 5 reports on a validation study of the Lifestyle Behavior Checklist (LBC), which is composed of two scales: the problem Scale (parental perceptions of the extent of their child's behavioral problems relating to childhood overweight) and the confidence scale (parental self-efficacy in managing the problem behaviors). In chapter 6 , the results of qualitative interviews are presented, which were conducted in order to identify barriers that Dutch youth health care professionals perceive when referring children to the obesity prevention intervention. Chapter 7 describes the design of the randomized controlled trial used to establish the effectiveness of the Lifestyle Triple $P$ intervention. Chapter 8 outlines both short- (4 months) and long-term (12 months) results of the trial. Finally, chapter 9 discusses the most important findings from the preceding chapters, and the theoretical and practical implications of the studies. 



\section{CHAPTER 2}

\section{General parenting, childhood overweight and obesity-inducing behaviors: a review}

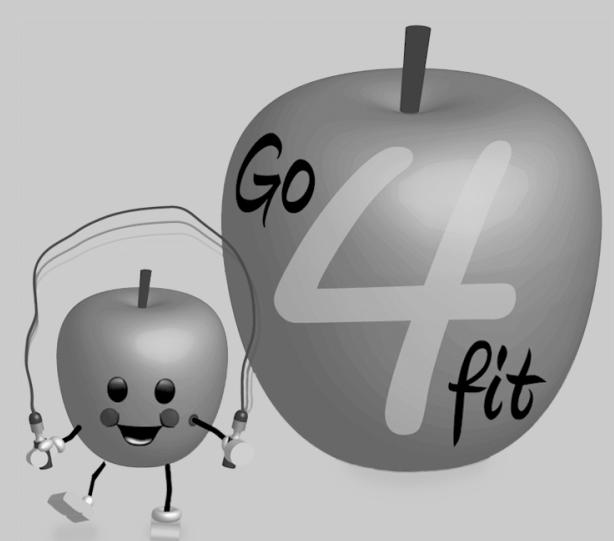

Published as: Sleddens, E.F.C., Gerards, S.M.P.L., Thijs, C., De Vries, N.K. and Kremers, S.P.J. (2011). General parenting, childhood overweight and obesity-inducing behaviors, a review. International Journal of Pediatric obesity, 6: e12-227 


\section{Abstract}

Despite emerging efforts to investigate the influence parents have on their children's weight status and related dietary and activity behaviors, reviews regarding the role of general parenting are lacking. We performed a systematic review regarding the relationship between general parenting and these weight-related outcomes to guide determinant research. In total, 36 studies were included in the review. Discrepancies across studies were found. Overall, however, results indicate that children raised in authoritative homes ate more healthily, were more physically active and had lower BMI levels, compared to children who were raised with other styles (authoritarian, permissive/indulgent, uninvolved/neglectful). Furthermore, findings of some moderation studies indicate that general parenting has a differential impact on children's weight status and related health behaviors, depending on child and parental characteristics. These findings underline the importance of acknowledging interactions between general parenting and both child and parent characteristics, as well as behaviorspecific parenting practices. 


\section{Introduction}

There has been a dramatic increase in prevalence of childhood overweight and obesity over the last few decades (Wang \& Lobstein, 2006). So-called energy balance-related behaviors (Kremers et al., 2005) contributing to excessive weight gain include the consumption of energy-dense foods, sugar-sweetened beverages (e.g., Du \& Feskens, 2010; Jebb, 2005) as well as low levels of physical activity and sedentary behaviors (e.g., Janssen et al., 2005; Malina \& Little, 2008). An area of emerging research focuses on the role of parents in the development of obesity-inducing health behaviors of their children. Many of these studies address the influence of parental feeding styles and specific parenting practices regarding food and/or activity (e.g., Brown, Ogden, Vogele, \& Gibson, 2008; Davison, Cutting, \& Birch, 2003; Hughes et al., 2007; Hughes, Power, Orlet Fisher, Mueller, \& Nicklas, 2005; Hughes, Shewchuk, Baskin, Nicklas, \& Qu, 2008; Joyce \& Zimmer-Gembeck, 2009; Matheson, Robinson, Varady, \& Killen, 2006; Mitchell, Brennan, Hayes, \& Miles, 2009; Musher-Eizenman, De Lauzon-Guillain, Holub, Leporc, \& Charles, 2009; Patrick, Nicklas, Hughes, \& Morales, 2005). Specific parenting practices include, for example, house-rules regarding breakfast consumption, parental control of child snacking and television viewing time. Existing reviews mainly concentrate on these specific types of parental influences affecting children's weight-related health outcomes, (e.g., Faith, Scanlon, Birch, Francis, \& Sherry, 2004; Wardle \& Carnell, 2007). Numerous efforts to unravel the influence of general parenting on children's weight-related behaviors suggest that the causal pathways are likely to be complex (Enten \& Golan, 2008; Golan \& Crow, 2004; Kitzmann, Dalton, \& Buscemi, 2008; Rhee, 2008; Ventura \& Birch, 2008). For instance, the contextual influence of general parenting is assumed to moderate the association between parenting practices and children's health outcomes (Darling \& Steinberg, 1993). The concept of general parenting has been defined as a constellation of attitudes and beliefs that create an emotional climate and determines behavioral expression between parent and child (Darling \& Steinberg, 1993). General parenting in this paper is also referred to as parenting style or dimensions (of parental behavior). In addition to having a potential moderating influence, general parenting may also impact on children's weight status through its influence on various parenting practices with regard to diet and physical (in)activity. Figure 2.1 depicts the possible mediating and moderating pathways of the influence of parenting on child weight (general parenting - child weight relationship).

The main objective of this review is to synthesize evidence regarding the influence of general parenting on children's diet and activity behaviors, and weight status. To our knowledge, this is the first review to focus exclusively on the influence of general parenting. 


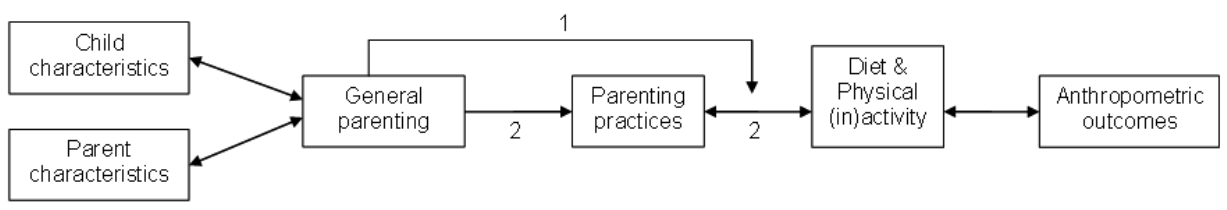

Figure 2.1: Conceptual model for the relationships between parenting and children's anthropometric-related outcomes

Note: Pathway 1 (moderation): interaction between general parenting and more specific parenting practices in predicting child diet \& physical (in)activity

Pathway 2 (mediation): influence of general parenting on child diet \& physical (in)activity mediated by effects of general parenting on more specific parenting practices

Child and parent characteristics may have an influence on all variables in the model.

\section{Background of parenting typologies}

The commonly used typological approach in parenting research is based on the work of Maccoby and Martin (1983), who described parenting style as a function of two dimensions of parental behavior: the extent to which parents are (1) responsive to their children's needs (responsiveness) and (2) controlling of their children's behaviors (demandingness). These two dimensions of parenting consistently emerge from factor analytic approaches. 'Responsiveness' has also been referred to as parental warmth (Baumrind, 1967; Becker, 1964; Schaefer, 1959), involvement (Grolnick \& Slowiaczek, 1994), nurturance (Block, 1981), child-centeredness (Pulkkinen, 1982), acceptance (Baumrind, 1967; Rohner, 1986), and caring/empathy (Parker, Tupling, \& Brown, 1979). 'Demandingness', on the other hand, is often related to aspects of control such as behavioral control (Schaefer, 1959) and firm control (Baumrind, 1965), restrictiveness (Becker, 1964), and democracy (Baldwin, 1948). By crossing the dimensions of responsiveness and demandingness, four prototypes of parenting are created (see Table 2.1) (Maccoby \& Martin, 1983): authoritative (parents who are both responsive and demanding), authoritarian (parents who are less responsive but highly demanding), indulgent or permissive (parents who provide a high level of responsiveness but are less demanding), and neglectful or uninvolved (parents who show relatively low levels of both dimensions).

Table 2.1: Fourfold typology of parenting based on the two-dimensional classification of Maccoby \& Martin (1983)

\begin{tabular}{lll}
\hline Demandingness & \multicolumn{2}{l}{ Responsiveness } \\
\cline { 2 - 3 } & High & Low \\
\hline High & Authoritative & Authoritarian \\
Low & Indulgent & Neglectful \\
\hline
\end{tabular}




\section{Methods}

\section{Search strategy and eligibility criteria}

Comprehensive literature searches were conducted between September 2009 and February 2010 utilizing a range of electronic databases (PubMed, PsycINFO, Scopus) together with lateral searching techniques (reference tracking and author searching). We included studies reporting general parenting and at least one of the following child outcomes: weight status, dietary intake (behaviors), physical (in)activity. To specify, literature searches were performed using at least one of the following parentingrelated keywords: parenting (style), (child) rearing, authoritative, authoritarian, permissive, indulgent, or neglectful; weight-related keywords: physical (in)activity, sedentary behavior, sport(s), television, computer, eating, diet, fruit, vegetable, breakfast, snack(ing), (sugar-sweetened) beverages, (over)weight, obesity, or Body Mass Index (BMI); and age-related keywords: infant, preschool, child, or adolescent. Other inclusion criteria were as follows: all studies should be written in English and published in a scientific peer-reviewed journal or as a dissertation; and the study sample should consist of infants, preschoolers, children or adolescents (here defined as children with an age below 18 years at baseline). No selection criteria with regard to study methodology were formulated. Studies of children with eating disorders were excluded, as well as studies assessing the relationship between general parenting and child eating styles without dietary intake outcomes. We included every eligible study published until February 2010, including e-publications. Using the selected keywords, 2244 papers were identified in Pubmed, PsychInfo and Scopus. Thereafter, all papers were screened on title, leading to 546 eligible hits. Of these, 434 were eliminated based on abstract evaluation. Full-text manuscripts were retrieved for the remaining 112 papers. This resulted in 33 studies which were considered eligible for inclusion. Furthermore, we applied reference tracking leading to 3 additional references. In total, 36 studies were considered eligible for the current review. Manuscripts were mainly excluded because they did not assess general parenting. Furthermore, prevention and intervention studies with regard to childhood overweight were excluded.

Two authors (E.S. and S.G.) independently screened all titles and abstracts of the manuscripts identified by the literature search for inclusion in this review. Full text versions of all potential relevant studies were obtained for further evaluation to determine inclusion, with any disagreement being resolved by discussion. In case of doubt, a third author (S.K.) was consulted. All studies selected for inclusion were scanned for additional references. Following this procedure, 36 publications were included in the review (Agras, Hammer, McNicholas, \& Kraemer, 2004; Berge, Wall, Bauer, \& Neumark-Sztainer, 2010a; Berge, Wall, Loth, \& Neumark-Sztainer, 2010b; Blissett \& Haycraft, 2008; Brann \& Skinner, 2005; Chen \& Kennedy, 2004; Chen \& 
Kennedy, 2005; Chen, Kennedy, Yeh, \& Kools, 2005; Chen, Unnithan, Kennedy, \& Yeh, 2008; De Bourdeaudhuij et al., 2009; Gable \& Lutz, 2000; Gibson et al., 2007; Hejazi, 2007; Hennessy, Hughes, Goldberg, Hyatt, \& Economos, 2010; Humenikova \& Gates, 2008; Kim, 2006; Kim et al., 2008; Kremers, Brug, De Vries, \& Engels, 2003; Lohaus, Vierhaus, \& Ball, 2009; Ludrosky, 2005; Lytle et al., 2003; Mendelson, White, \& Schliecker, 1995; Moens, Braet, \& Soetens, 2007; Musher-Eizenman \& Holub, 2006; Mustillo et al., 2003; Olvera \& Power, 2010; Pearson, Atkin, Biddle, Gorely, \& Edwardson, 2010; Reineke, 2008; Rhee, Lumeng, Appugliese, Kaciroti, \& Bradley, 2006; Schmitz et al., 2002; Topham et al., 2010; Van der Horst et al., 2007; Vereecken, Legiest, De Bourdeaudhuij, \& Maes, 2009; Wake, Nicholson, Hardy, \& Smith, 2007; West, 2007; Zeller, Boles, \& Reiter-Purtill, 2008). Figure 2.2 depicts the number of all studies published up till 2010 regarding the general parenting - child weight relationship. This figure shows that the number of studies examining this relationship has increased in recent years; from two studies before 2002 to about 9 studies published in 2008 and 2009 together.

\section{Data extraction}

Data regarding sample characteristics (sample size, child age, gender, ethnicity, location and setting), measurements (overview of instruments assessing general parenting, child dietary and physical (in)activity behavior, child weight status), and study results were abstracted by the first author (E.S.) and checked by the second author (S.G.). Instruments measuring independent variables other than parenting styles, such as parenting practices, were only described when interaction was tested with general parenting in predicting children's weight-related outcomes. Studies assessing interaction could be valuable in understanding the complex mechanisms behind the general parenting - child weight relationship (see Figure 2.1). We report on results of studies with a-priori hypotheses about possible interaction and on results of post-hoc analyses (i.e. interaction patterns that were not specified at the beginning of the study). The results of the reviewed studies are presented in chronological order in the Supplementary Table. For all studies, both statistically significant results (depicted with closed spheres) and non-significant results (depicted with open spheres) are reported to give a complete overview of the associations between all study variables. 


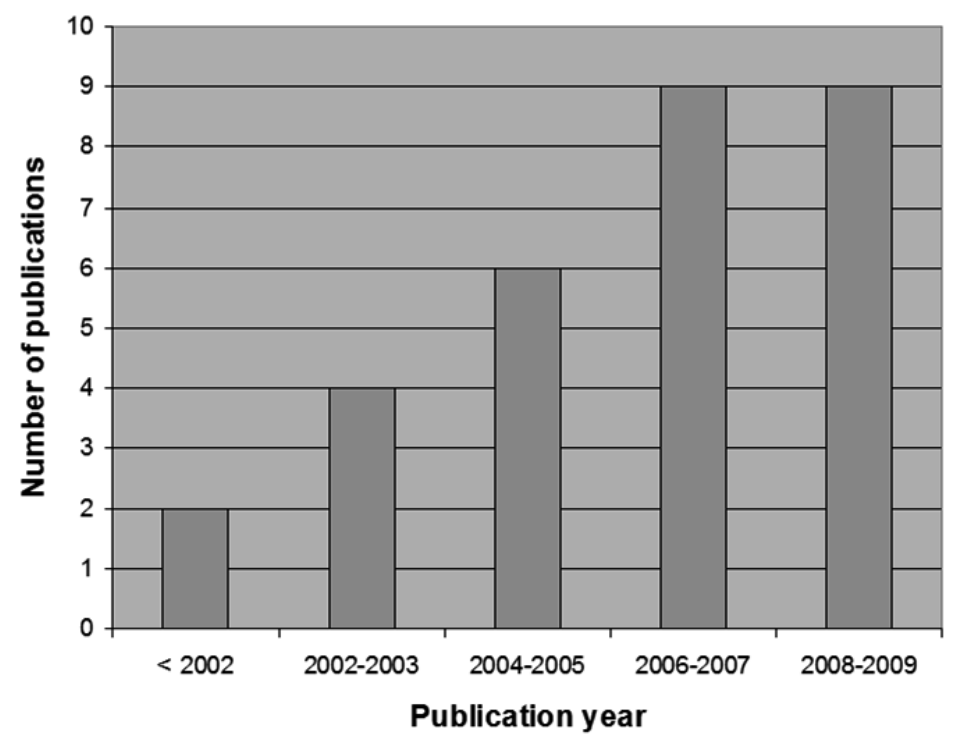

Figure 2.2: Number of publications examining the general parenting - child weight relationship by year $(\mathrm{N}=30)$

Note: All studies regarding the general parenting - child weight relationship published in a scientific journal issue (no e-publications) before 2010 are included in this figure.

\section{Results}

\section{Study characteristics of the included studies}

The sample size of the included studies ranged from 45 to over 4000, representing the absolute number of caregivers and/or children who participated in the study. Different study characteristics in terms of sample size, age, gender, ethnicity and location/setting of the study are depicted in Table 2.2. Most study populations consisted of North-Americans ( $n=23)$, followed by Western Europeans $(n=9)$, Australians $(n=3)$, Asians $(n=3)$, Southern Europeans $(n=2)$ and Eastern Europeans $(n=1)$. Samples from the United States consisted of ethnically diverse populations, including participants with Hispanic, African and/or Asian backgrounds. 


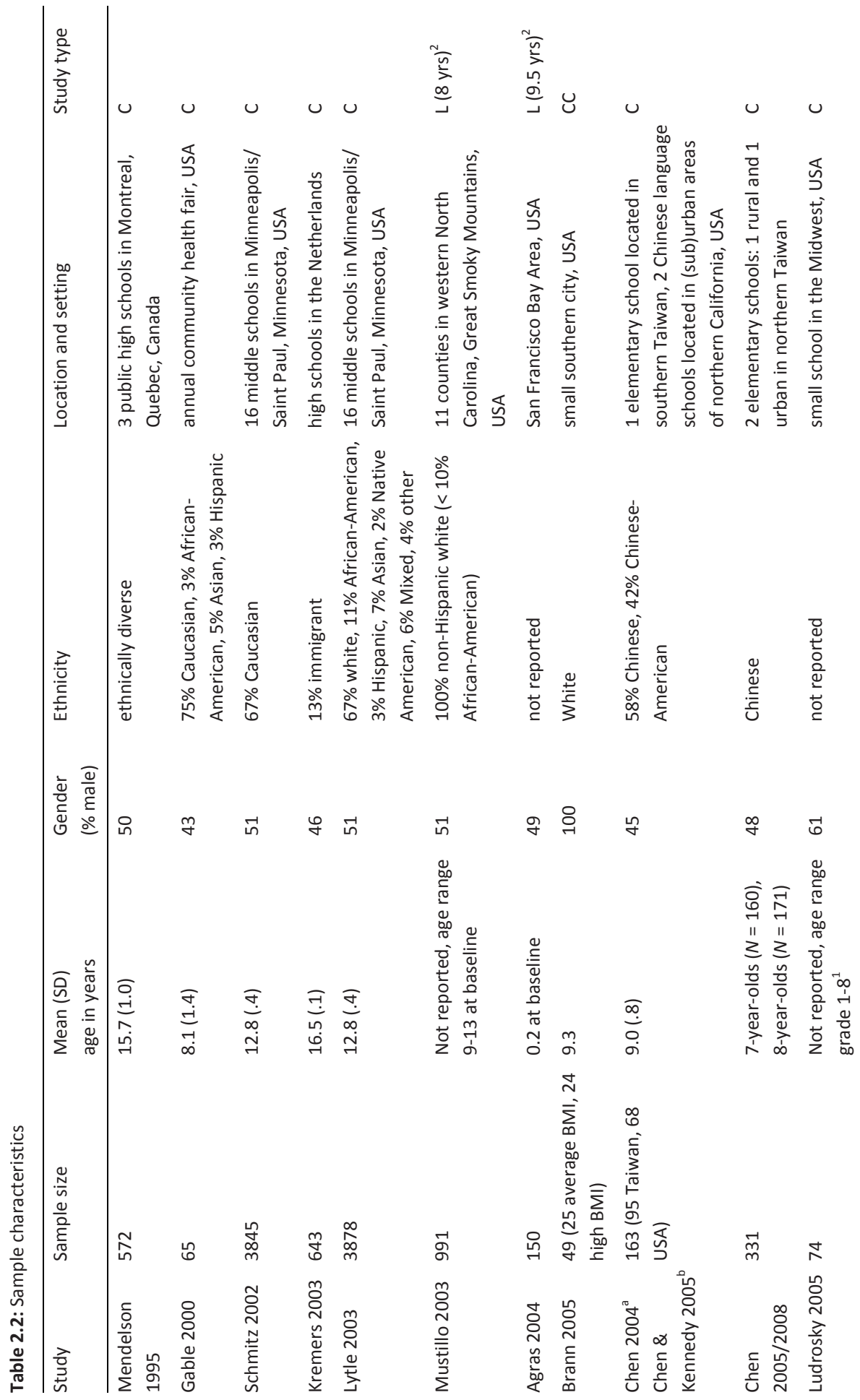


RELATIONSHIP BETWEEN PARENTING AND CHILD WEIGHT I 23

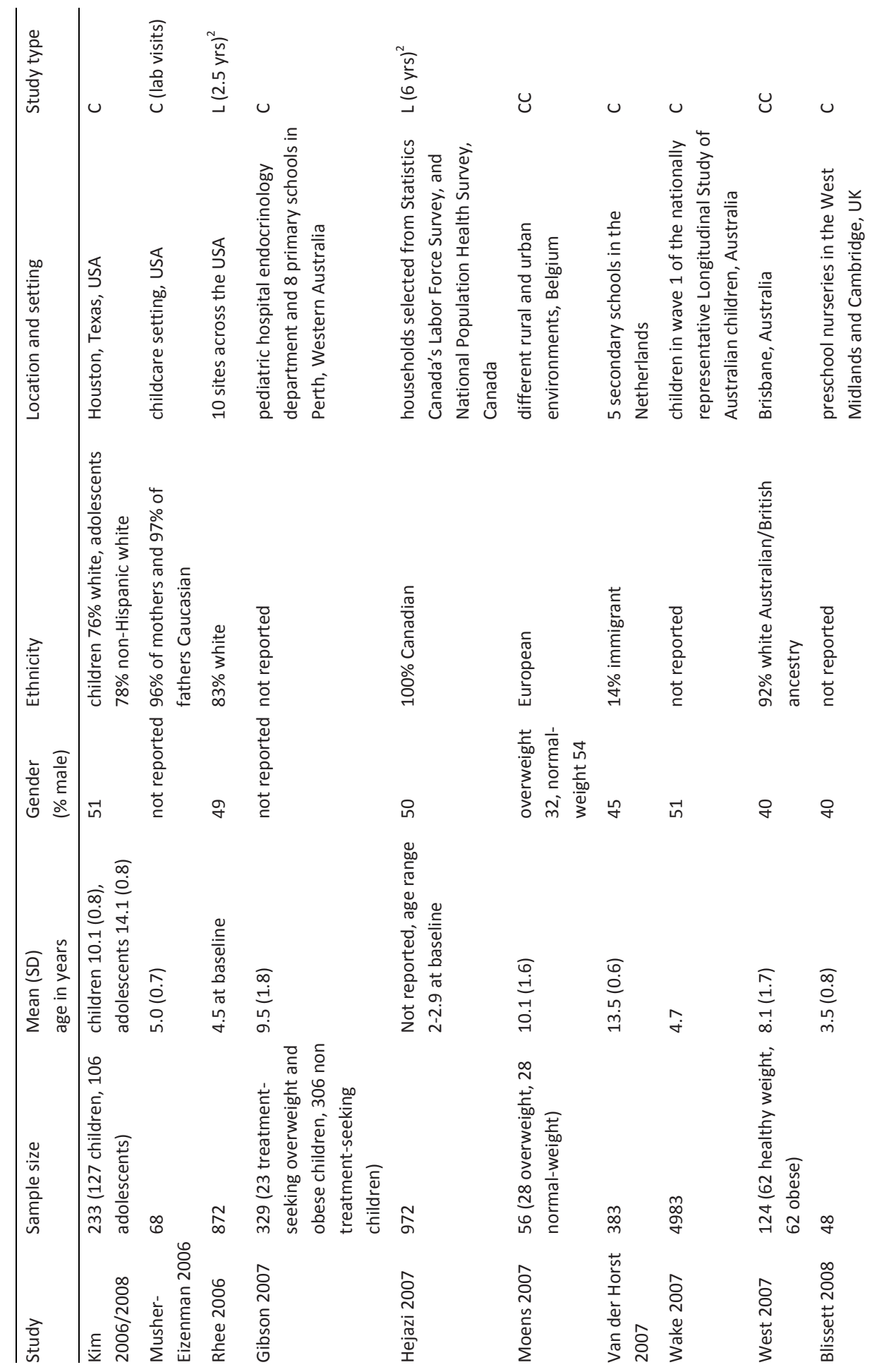




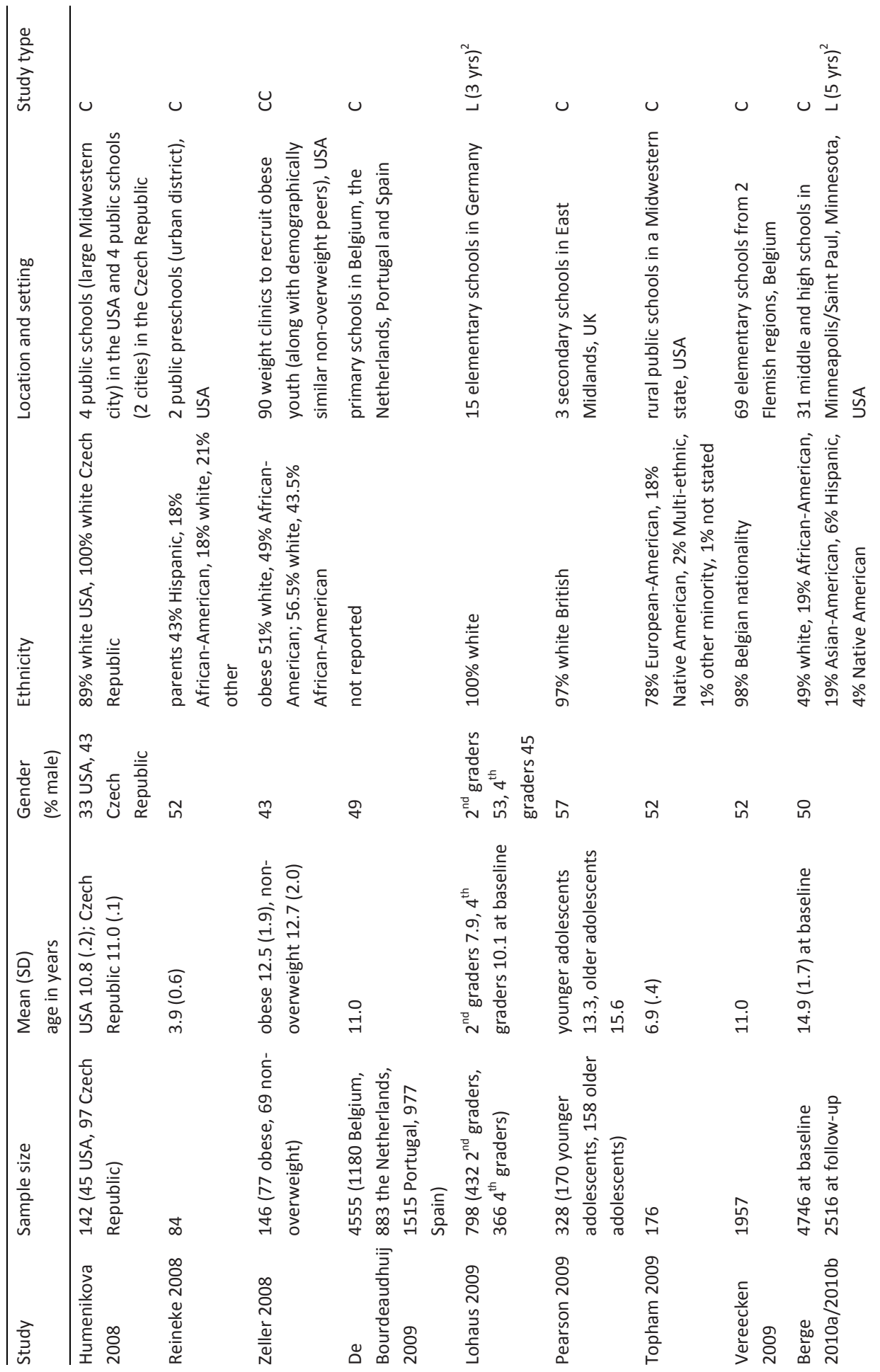


RELATIONSHIP BETWEEN PARENTING AND CHILD WEIGHT I 25

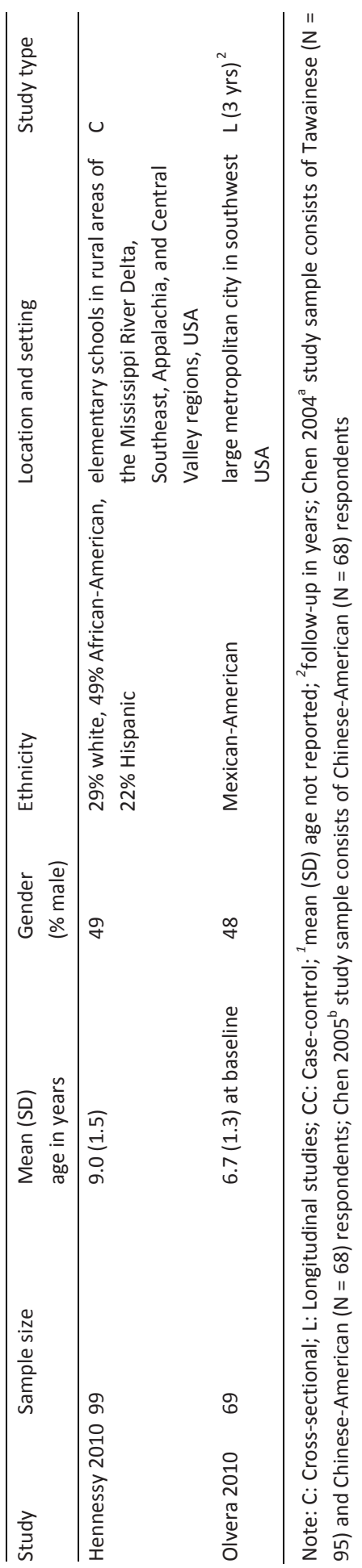




\section{Parenting measures}

In total, 21 different instruments were used to measure parenting dimensions or styles (see online supplement). All of these instruments have proven to be valid and reliable. The parenting tools which are used most often are the 'Child Rearing Practices Report' (Block, 1981) applied in four studies (Chen et al., 2005; Chen et al., 2008; Gable \& Lutz, 2000; Ludrosky, 2005), and the 'Parenting Style Instrument' (Den Exter Blokland, Engels, \& Finkenauer, 2001; Lamborn, Mounts, Steinberg, \& Dornbusch, 1991; Steinberg, Elmen, \& Mounts, 1989) also used in four studies (De Bourdeaudhuij et al., 2009; Kremers et al., 2003; Pearson et al., 2010; Van der Horst et al., 2007). The 'Parenting Practices Questionnaire' (Robinson, Mandleco, Olsen, \& Hart, 1995) or its short form, the 'Parenting Styles and Dimensions Questionnaire' (Robinson, Mandleco, Olsen, \& Hart, 2001) were administered to participants in three studies (Blissett \& Haycraft, 2008; Brann \& Skinner, 2005; Topham et al., 2010). Several parenting instruments were applied in two studies, i.e., the 'Authoritative Parenting Index' (Jackson, Bee Gates, \& Henriksen, 1994) used by Schmitz et al. (2002) and Lytle et al. (2003); the 'Parental Authority Questionnaire' (Buri, 1991) used by Agras et al. (2004) or its revised version (Reitman, Rhode, Hupp, \& Altobello, 2002) used by Musher-Eizenman and Holub (2006); the 'Parenting Dimension Inventory' (Power, 1993) used by Olvera and Power (2010) and Hennessy et al. (2010); the 'Attitudes Toward Child-Rearing Scales' (Croake \& Hinkle, 1991) used by Chen and Kennedy (2004, 2005); and the 'Parenting Scale' (Arnold, O'Leary, Wolff, \& Acker, 1993) used by Gibson et al. (2007) and West (2007). For an overview regarding reporting of general parenting and for a brief description of all parenting instruments used in the included studies, we refer to Table 2.3 and the Supplement on pages 45-61, respectively.

\section{Findings per outcome variable}

The included studies were clustered by outcome variable: dietary behavior $(n=14)$, physical (in)activity $(n=10)$, and weight status $(n=29)$. Below, we give an overview of the key findings. Further study details are presented in Tables 2.3, 2.4 and the supplement on pages 45-61.

\section{Dietary behavior}

Eleven cross-sectional studies (Chen \& Kennedy, 2005; De Bourdeaudhuij et al., 2009; Kim, 2006; Kim et al., 2008; Kremers et al., 2003; Ludrosky, 2005; Lytle et al., 2003; Musher-Eizenman \& Holub, 2006; Pearson et al., 2010; Van der Horst et al., 2007; Vereecken et al., 2009) and three longitudinal studies (Agras et al., 2004; Berge et al., 2010b; Lohaus et al., 2009) measured the relationship between parenting and children's weight-related dietary behaviors (see Table 2.4a). 
RELATIONSHIP BETWEEN PARENTING AND CHILD WEIGHT I 27

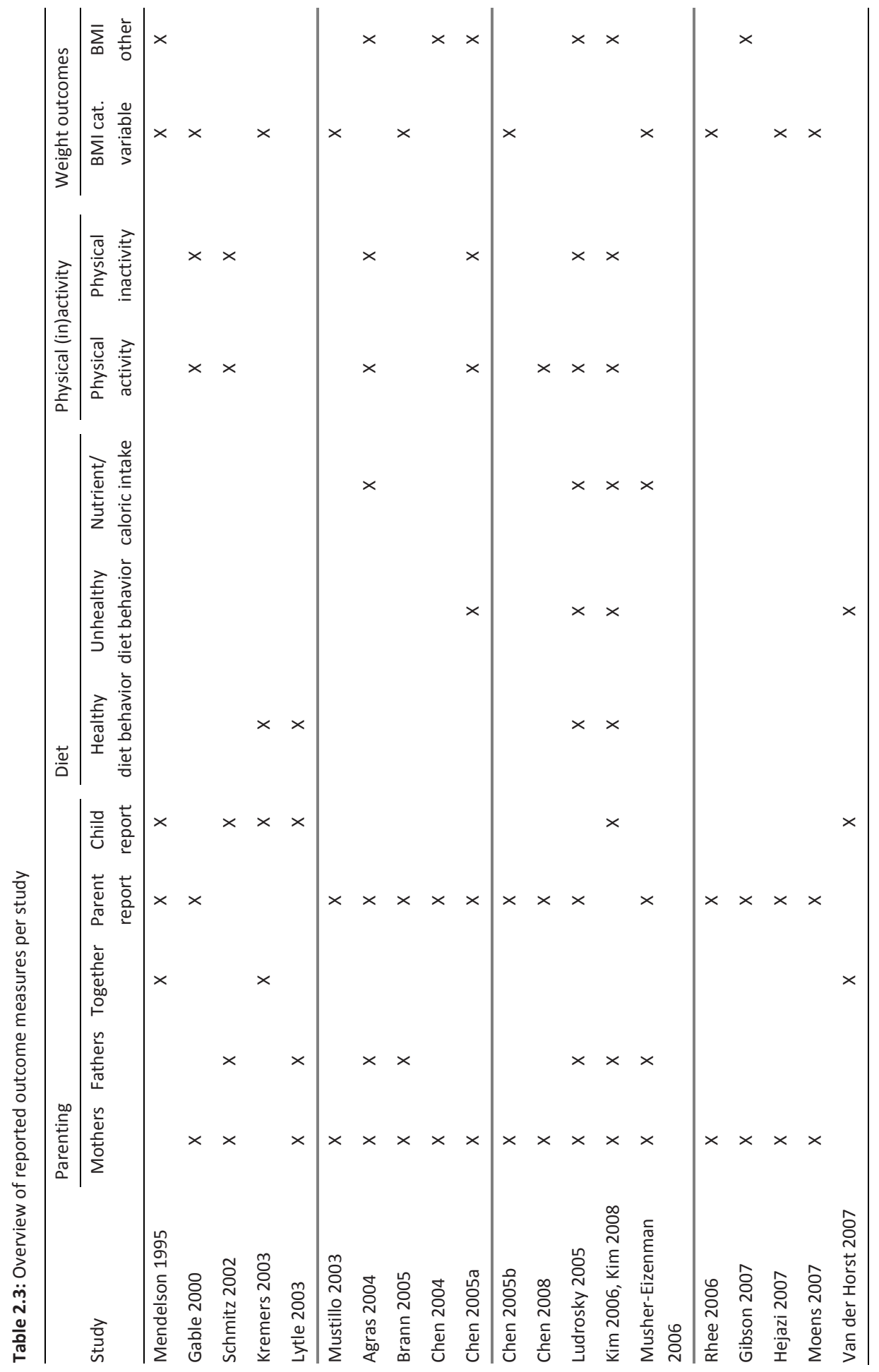


28 CHAPTER 2

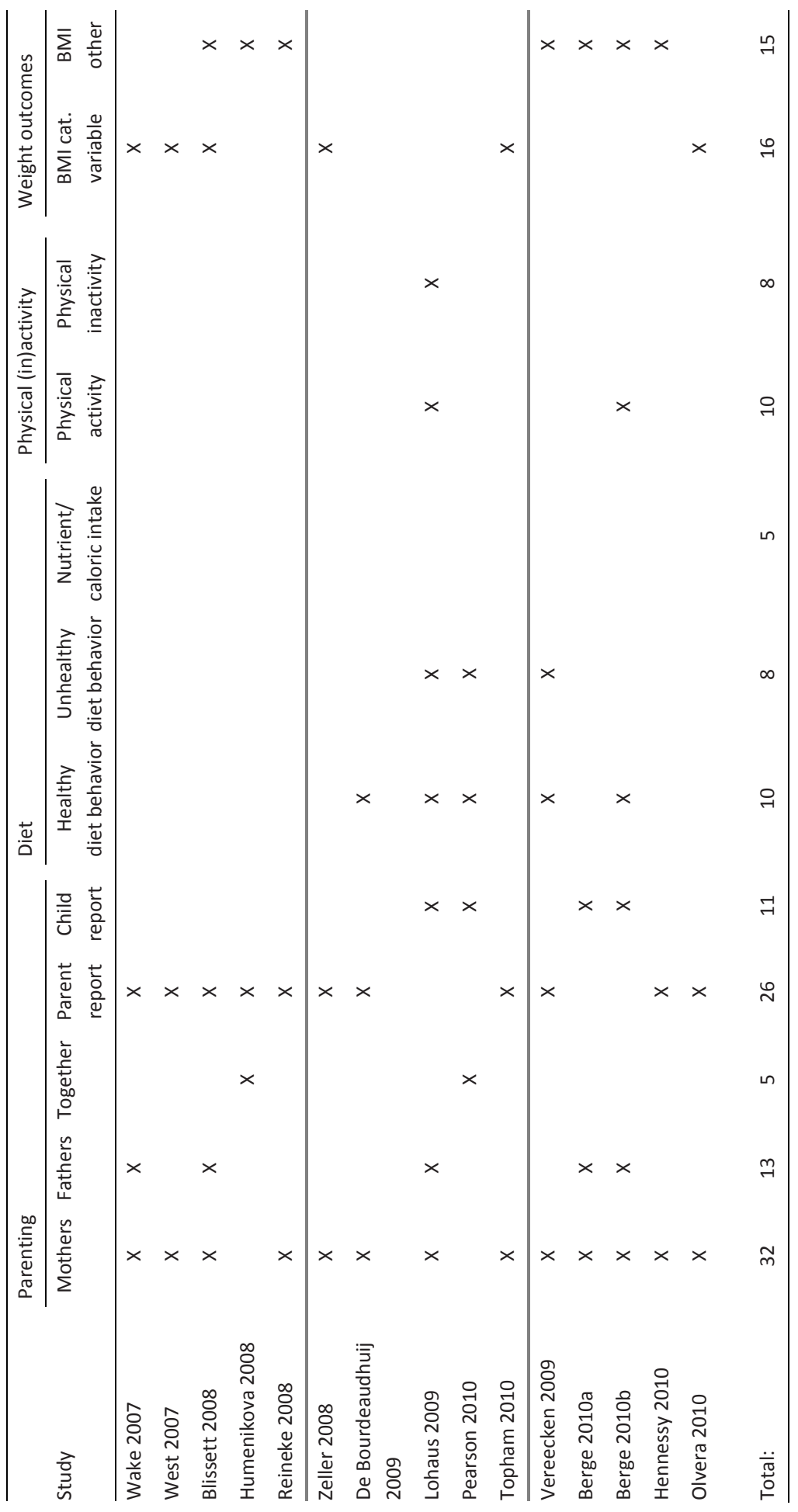




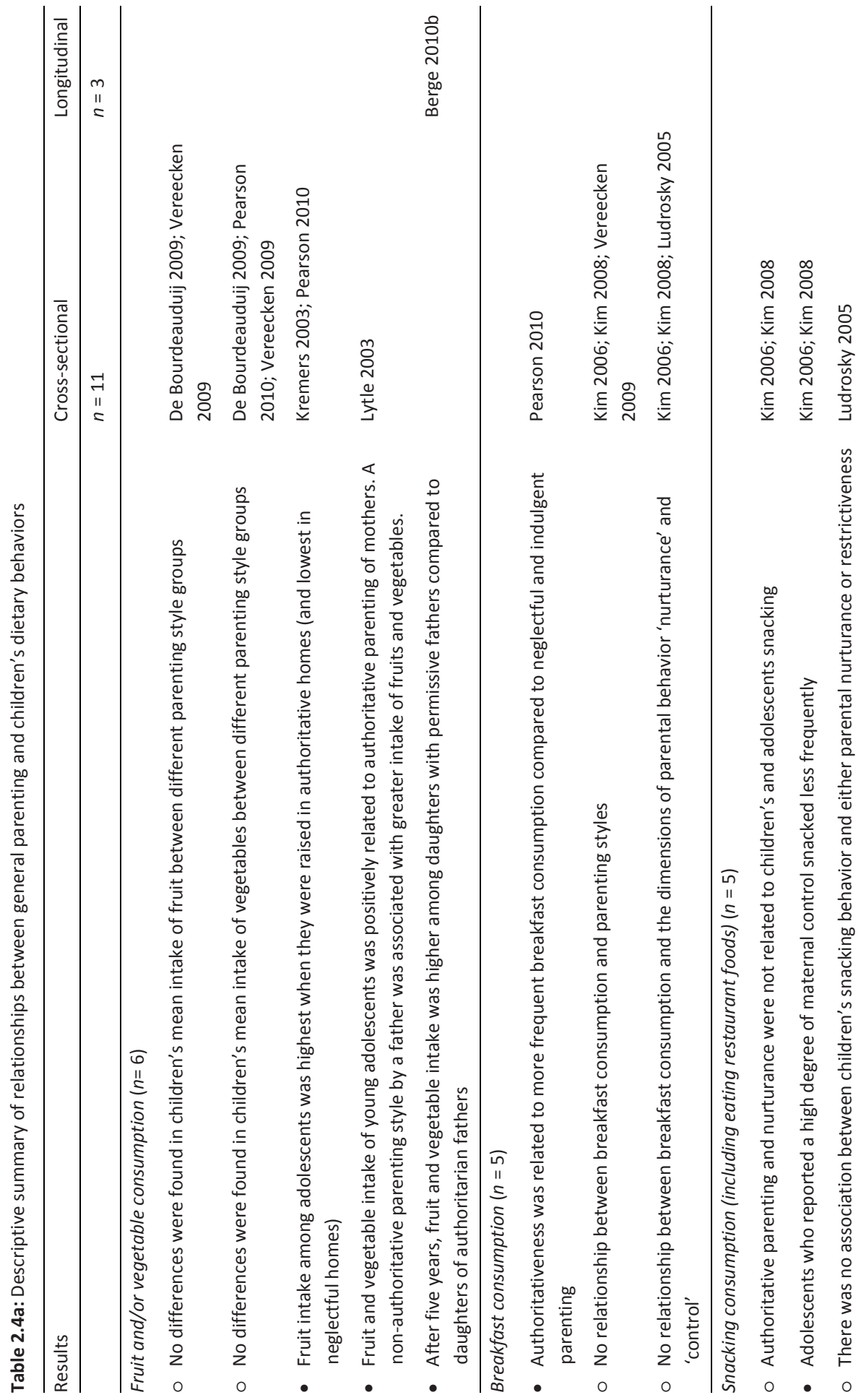




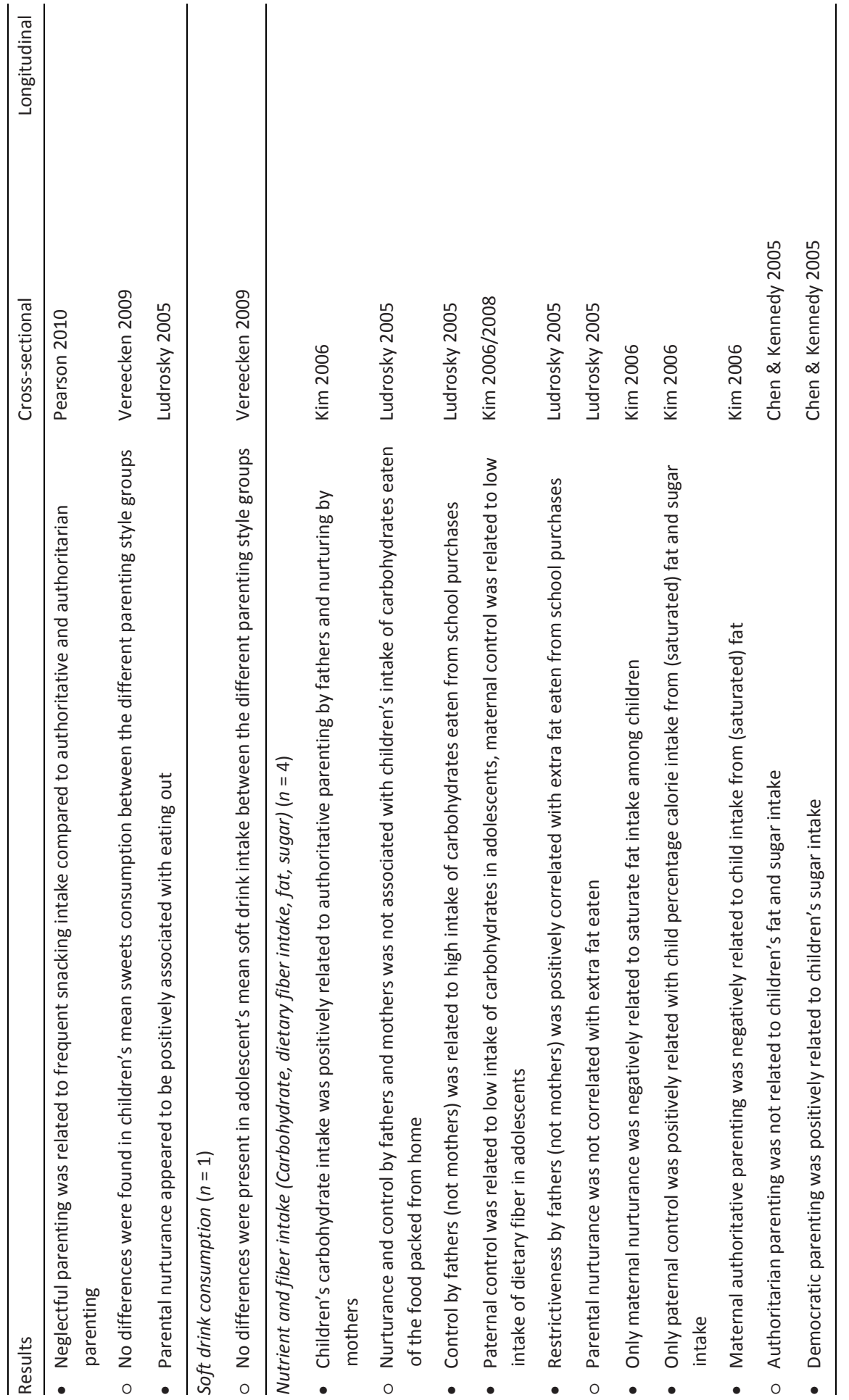


RELATIONSHIP BETWEEN PARENTING AND CHILD WEIGHT I 31

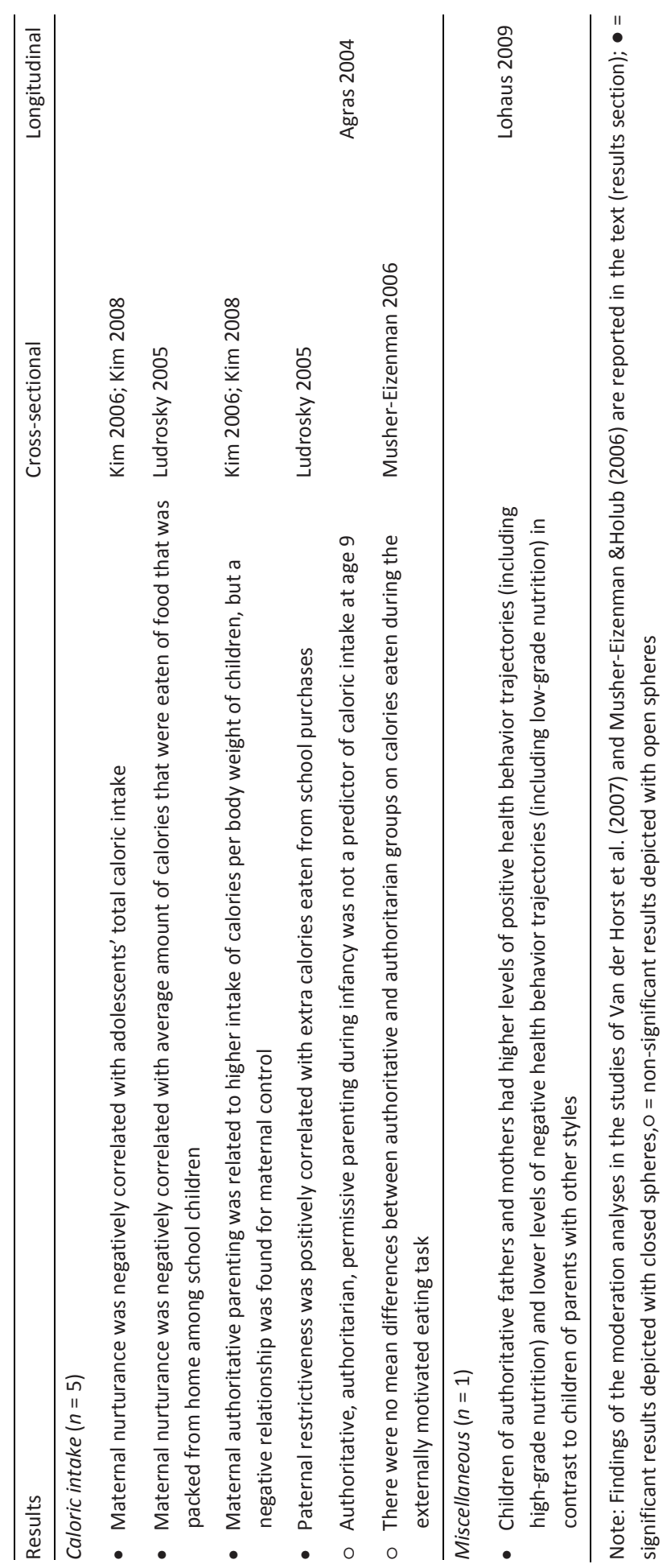


Fruit and/or vegetable consumption. In two large scale cross-sectional studies (De Bourdeaudhuij et al., 2009; Vereecken et al., 2009), one including multiple countries (De Bourdeaudhuij et al., 2009), no relationships were found with parenting styles. Other studies found favorable effects of authoritative parenting on fruit intake (Kremers et al., 2003; Lytle et al., 2003; Pearson et al., 2010); in the study of Lytle et al. (2003) this was only true for mothers, and this positive relationship was also present for vegetable intake. Berge et al. (2010b), the only study using a longitudinal design, found different results; daughters of permissive fathers having higher intakes of fruit and vegetables five years later than those of authoritarian fathers.

Breakfast consumption. For the relationship between general parenting and breakfast consumption inconsistent results are reported. Pearson et al. (2010) indicated that authoritative parenting was related to more frequent breakfast consumption compared to neglectful and indulgent parenting. Contrary, other studies found no relationship of breakfast consumption with parenting styles (Kim, 2006; Kim et al., 2008; Vereecken et al., 2009) or the dimensions of parental behavior 'nurturance' and 'control' (Kim, 2006; Kim et al., 2008; Ludrosky, 2005).

Snacking and soft drink consumption. Snacking was uncorrelated to most parenting styles and dimensions (Kim, 2006; Kim et al., 2008; Ludrosky, 2005). Only adolescents who reported a high degree of maternal control snacked less frequently (Kim, 2006; Kim et al., 2008). Neglectful parenting was related to frequent snacking compared to authoritative and authoritarian parenting (Pearson et al., 2010). Vereecken et al. (2009), who besides sweets consumption also assessed soft drink consumption, reported that no associations were present between these overweight inducing behaviors and parenting styles as defined using the four-fold typology.

Van der Horst et al. (2007) executed moderation analyses, examining whether restrictive feeding practices have a different effect on adolescents' sugar-sweetened beverage consumption depending on the parenting style of their caregivers. Results indicated that the parenting dimensions of 'involvement' and 'strictness' modified the associations between restrictive feeding and sugar-sweetened beverage consumption, in a sense that controlling parenting practices had the strongest association with a decreased consumption of these drinks when parents were moderately controlling and highly involved.

Nutrient and fiber intake. Kim and colleagues (2006) found that children's carbohydrate intake was positively related with authoritative parenting by fathers and nurturance by mothers. Inconsistent findings were found for controlling parenting; this was related either to high (Ludrosky, 2005) or low (Kim, 2006; Kim et al., 2008) intake of 
carbohydrates or fiber. For fat intake, there was a negative relationship with nurturing and authoritative parenting by mothers, whereas a positive relationship was found with fathers' controlling parenting (Kim, 2006; Kim et al., 2008; Ludrosky, 2005). In a study of Chinese-American children, Chen et al. (2005) indicated that a positive association was found between democratic parenting and sugar intake.

Caloric intake. In general, caloric intake was negatively correlated with maternal nurturance (Chen et al., 2008; Kim, 2006; Ludrosky, 2005), but positively with parental restrictiveness (Chen et al., 2008). In the longitudinal study of Agras et al. (2004) authoritative, authoritarian or permissive parenting during infancy was not significantly related to caloric intake of children at 9.5 years.

Musher-Eizenman and Holub (2006) conducted moderation analyses to find out whether parenting style would moderate the effects of restrictive feeding practices on children's caloric intake through externally motivated eating. The authors hypothesized that authoritarian parenting is related to high levels of caloric intake among children, whereas authoritative parenting is expected to attenuate the negative effects of restrictive feeding. In this small sample study, an external eating task was performed to assess eating in the absence of hunger and ultimately caloric intake. The results of this study showed that fathers with an authoritative parenting style who applied restrictive feeding practices had a protective effect on their child's caloric intake (i.e. associated with low caloric intake), whereas mothers with an authoritarian parenting style who applied these restrictive feeding practices had a counterproductive effect on caloric intake (i.e. associated with high caloric intake).

Positive and negative health behaviors. Results of a longitudinal study revealed that authoritative fathers and mothers had children with higher levels of positive health behavior trajectories (including high-grade nutrition such as fruit and vegetable consumption) and lower levels of negative health behavior trajectories (including lowgrade nutrition) over a three-year period in contrast to children of parents with other styles (Lohaus et al., 2009). However, it should be noted that the positive healthrelated behavior measure used in this study also incorporated questions regarding physical activity, use of health care and personal hygiene. The negative health-related behavior measure also included statements regarding television viewing, nicotine and alcohol consumption, and risk behavior.

Physical (in)activity

Seven cross-sectional studies (Chen \& Kennedy, 2005; Chen et al., 2008; Gable \& Lutz, 2000; Kim, 2006; Kim et al., 2008; Ludrosky, 2005; Schmitz et al., 2002) and three lon- 
gitudinal studies (Agras et al., 2004; Berge et al., 2010b; Lohaus et al., 2009) examined associations between general parenting and children's physical (in)activity levels (see Table 2.4b). The cross-sectional studies reported inconsistent results regarding the parenting - physical activity relationship. In the studies assessing parental control no associations were revealed with children's and/or adolescent's physical (in)activity levels (Kim, 2006; Kim et al., 2008; Ludrosky, 2005). In some studies no associations were found between authoritarian parenting (Chen \& Kennedy, 2005; Gable \& Lutz, 2000), non-authoritative parenting (Kim, 2006; Kim et al., 2008), authoritative parenting (Gable \& Lutz, 2000), democratic parenting (Chen \& Kennedy, 2005) and child physical (in)activity. The more positive parenting variables (e.g., nurturance and authoritative parenting) were more often positively associated with activity levels (Kim, 2006; Kim et al., 2008; Ludrosky, 2005).

Two cross-sectional studies found that the relationship between general parenting and child activity was influenced by gender (Chen et al., 2008; Schmitz et al., 2002). However, mixed results were found. Chen et al. (2008) who conducted a study in Taiwan found that physical activity was positively associated with authoritative parenting in 7- and 8-year-old boys, but with authoritarian parenting in girls of the same age. Schmitz and colleagues (2002) found different results among a large group of young adolescents. Only for female adolescents, maternal authoritativeness was a significant positive predictor of physical activity and a negative predictor of sedentary leisure habits.

Findings of the longitudinal studies indicated that authoritative parenting was a positive predictor of physical activity (Berge et al., 2010b; Lohaus et al., 2009), a negative predictor of sedentary leisure-time activities (leisure-time behaviors which require very little energy, including television viewing) (Lohaus et al., 2009) or a non-significant predictor (Agras et al., 2004) of physical (in)activity (including television viewing and assessment of physical activity via accelerometry) at follow-up. Berge et al. (2010b) showed that only for adolescent sons, authoritative parenting by fathers predicted frequent physical activity at five-year follow-up in comparison with sons of neglectful fathers. 
RELATIONSHIP BETWEEN PARENTING AND CHILD WEIGHT I 35

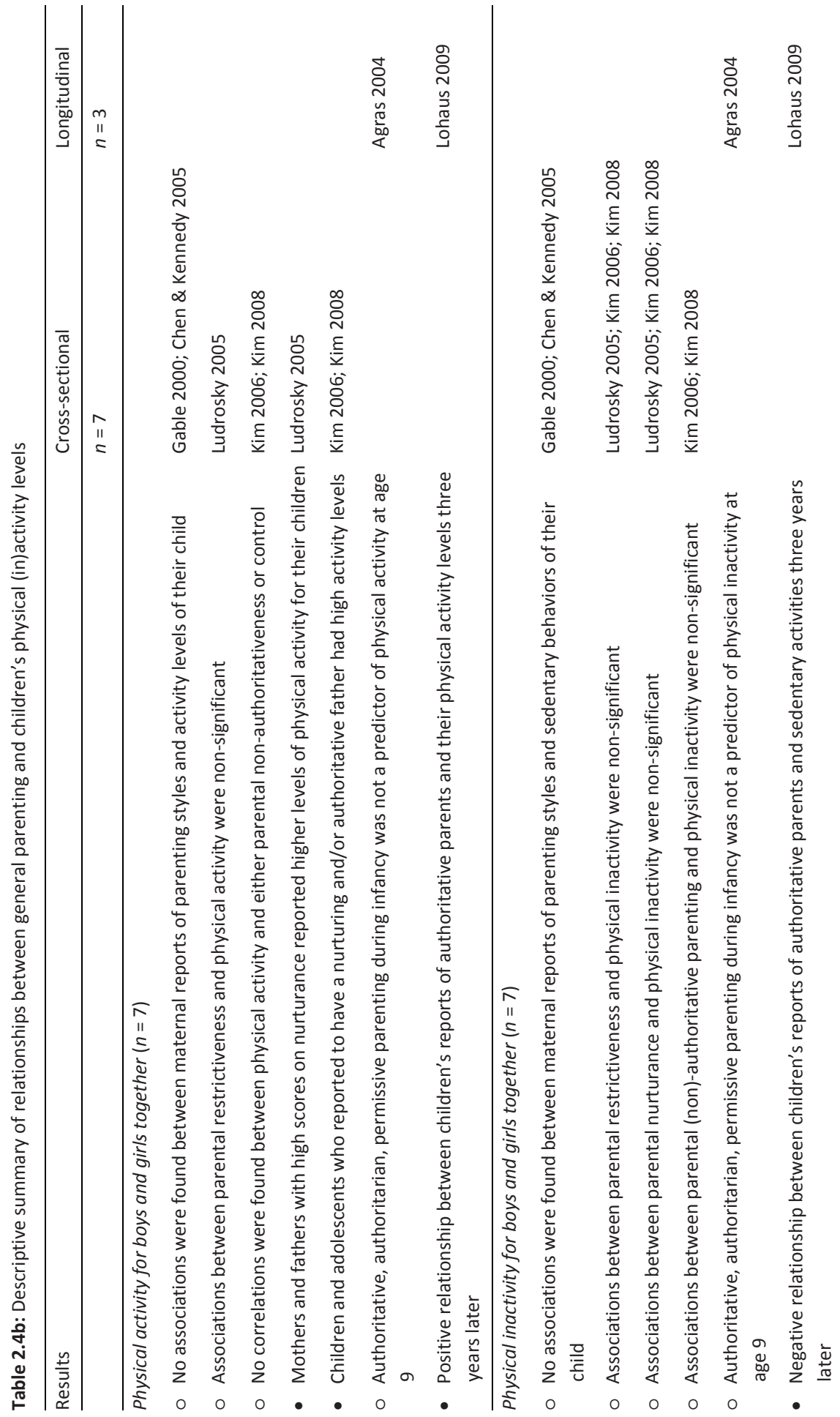


36 | CHAPTER 2

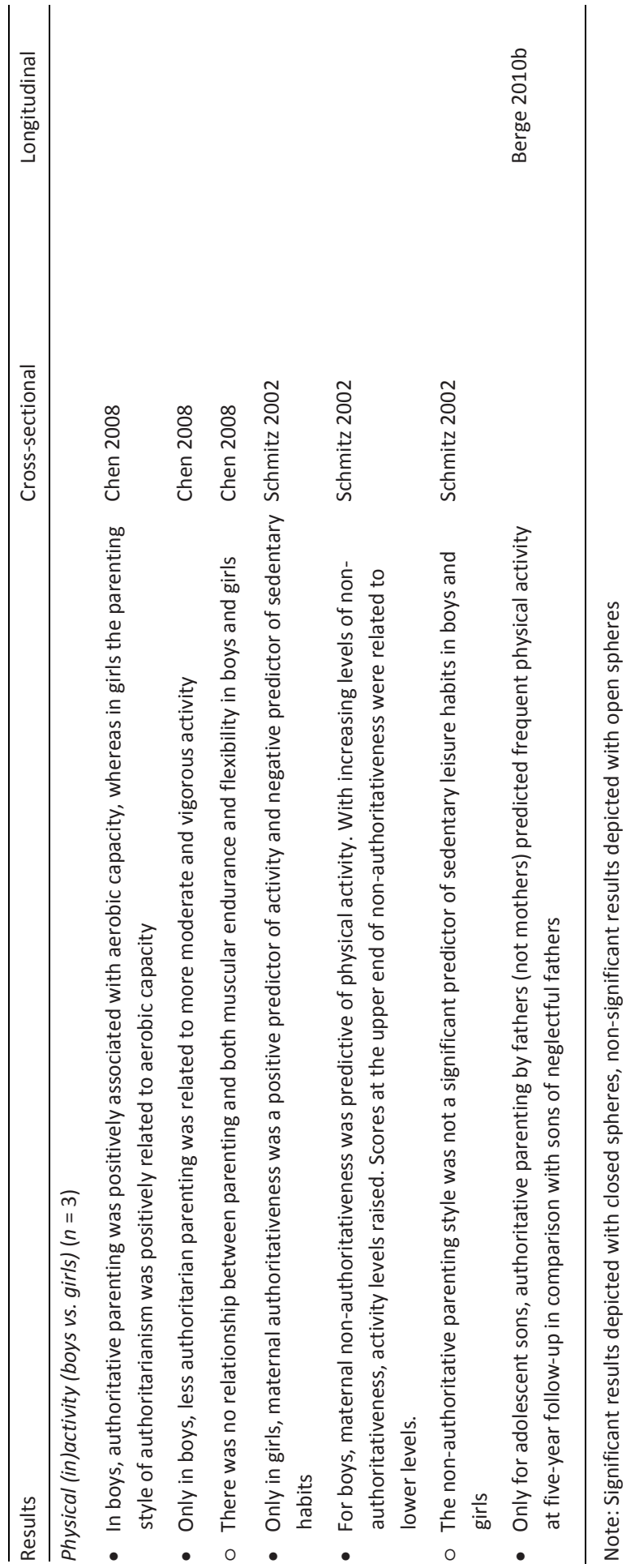




\section{Weight status}

In total, 29 studies were identified which examined relationships between general parenting and a weight-related outcome variable (see Table $2.4 \mathrm{c}$ ). The majority of these studies used cross-sectional $(n=19)$ (Berge et al., 2010a; Blissett \& Haycraft, 2008; Chen \& Kennedy, 2004; Chen \& Kennedy, 2005; Chen et al., 2005; Gable \& Lutz, 2000; Gibson et al., 2007; Hennessy et al., 2010; Humenikova \& Gates, 2008; Kim, 2006; Kim et al., 2008; Kremers et al., 2003; Ludrosky, 2005; Mendelson et al., 1995; Musher-Eizenman \& Holub, 2006; Reineke, 2008; Topham et al., 2010; Vereecken et al., 2009; Wake et al., 2007) or case-control ( $n=4)$ (Brann \& Skinner, 2005; Moens et al., 2007; West, 2007; Zeller et al., 2008) rather than a longitudinal design $(n=6)$ (Agras et al., 2004; Berge et al., 2010b; Hejazi, 2007; Mustillo et al., 2003; Olvera \& Power, 2010; Rhee et al., 2006).

Six cross-sectional studies found no significant effects of child weight status group (Blissett \& Haycraft, 2008; Brann \& Skinner, 2005; Chen et al., 2005; Gable \& Lutz, 2000; Mendelson et al., 1995; Moens et al., 2007) on general parenting variables. Some other cross-sectional studies found no mean differences in child BMI between various parenting style groups (Kremers et al., 2003; Musher-Eizenman \& Holub, 2006). However, two studies comparing parenting styles of mothers with obese and normal-weight children did report significant results (West, 2007; Zeller et al., 2008), indicating mothers of obese children scoring higher on 'laxness' and 'overreactivity' (West, 2007) and lower on 'behavioral control' (Zeller et al., 2008).

Many cross-sectional studies reported some non-significant findings regarding associations between particular parenting dimensions or styles and children's BMI (Berge et al., 2010a; Blissett \& Haycraft, 2008; Chen \& Kennedy, 2004; Chen \& Kennedy, 2005; Gibson et al., 2007; Hennessy et al., 2010; Humenikova \& Gates, 2008; Kim, 2006; Kim et al., 2008; Ludrosky, 2005; Reineke, 2008; Vereecken et al., 2009; Wake et al., 2007). Across studies which found significant relationships, inconsistent findings were reported. Some studies found that authoritative parenting was associated with lower BMI values (Kim, 2006; Ludrosky, 2005; Van der Horst et al., 2007; Zeller et al., 2008). In contrast, Humenikova and Gates (2008) found that less authoritative parenting was related with lower BMI z-scores in Czech children. Permissive parenting in US children (Humenikova \& Gates, 2008) and democratic parenting in both Taiwanese and Chinese-American children (Chen \& Kennedy, 2004; Chen \& Kennedy, 2005) were positively related to children's BMI z-scores. Other studies found that parenting control of mothers (Kim, 2006; Kim et al., 2008) and fathers (Ludrosky, 2005) (e.g., including forms of authoritarian and psychological control) was positively related to BMI z-score. However, Wake et al. (2007) reported an inverse relationship between paternal control (some aspects of behavioral control) and child BMI. 
It seems that relationships which were found depended on characteristics of the outcome variable. In general, no association between general parenting and weight status was found when a categorical variable was used; more often, significant relationships were found when BMI was used as a continuous outcome variable.

Six longitudinal studies were identified assessing whether general parenting predicts weight status at follow-up or weight status development among children (Agras et al., 2004; Berge et al., 2010b; Hejazi, 2007; Mustillo et al., 2003; Olvera \& Power, 2010; Rhee et al., 2006). Three of these studies found positive effects of authoritative parenting on children's weight status (i.e. authoritative parenting was related with lower weight at follow-up) (Berge et al., 2010b; Olvera \& Power, 2010; Rhee et al., 2006). One study relating parenting styles to child weight status, failed to detect significant effects (Agras et al., 2004). In two studies, children's weight and height were repeatedly measured to define various developmental BMI trajectories (Hejazi, 2007; Mustillo et al., 2003). The aim of these studies was to examine whether there were any differences in parenting dimensions between these defined groups. Only one study reported significant differences (Hejazi, 2007).

A minority of existing (cross-sectional) studies focused on assessing interaction between variables (i.e., parenting styles, parent or child characteristics) in predicting children's weight status (Berge et al., 2010a; Topham et al., 2010; Zeller et al., 2008). Zeller et al. (2008) tested whether parenting interacted with child temperament in predicting child weight status. Findings showed that interaction was present between low maternal warmth and difficult child temperament, indicating that $69 \%$ of obese youth were classified as being high on difficult temperament and low on maternal warmth as compared to $31 \%$ of non-overweight youth (Zeller et al., 2008). Topham and colleagues (2010) assessed the role of other potential moderating factors (i.e., Socio-Economic Status (SES) and maternal depression) on the relationship between general parenting style (authoritative, authoritarian, permissive) and child obesity. They hypothesized that maternal depression and high SES would aggravate the impact of authoritarian parenting as well as permissive parenting on child obesity. Findings indicated that only for permissive parenting there was an interaction with maternal depression and SES. Both depressed mothers and high SES mothers had children who are more likely to be obese when they had permissive parenting styles. Finally, Berge et al. (2010a) found in a large ethnically diverse group of US teens, who reported about the parenting styles of their parents, that the combination of maternal authoritarian parenting and paternal neglectful parenting was related with a high BMI in sons, but not in daughters. 
RELATIONSHIP BETWEEN PARENTING AND CHILD WEIGHT I 39

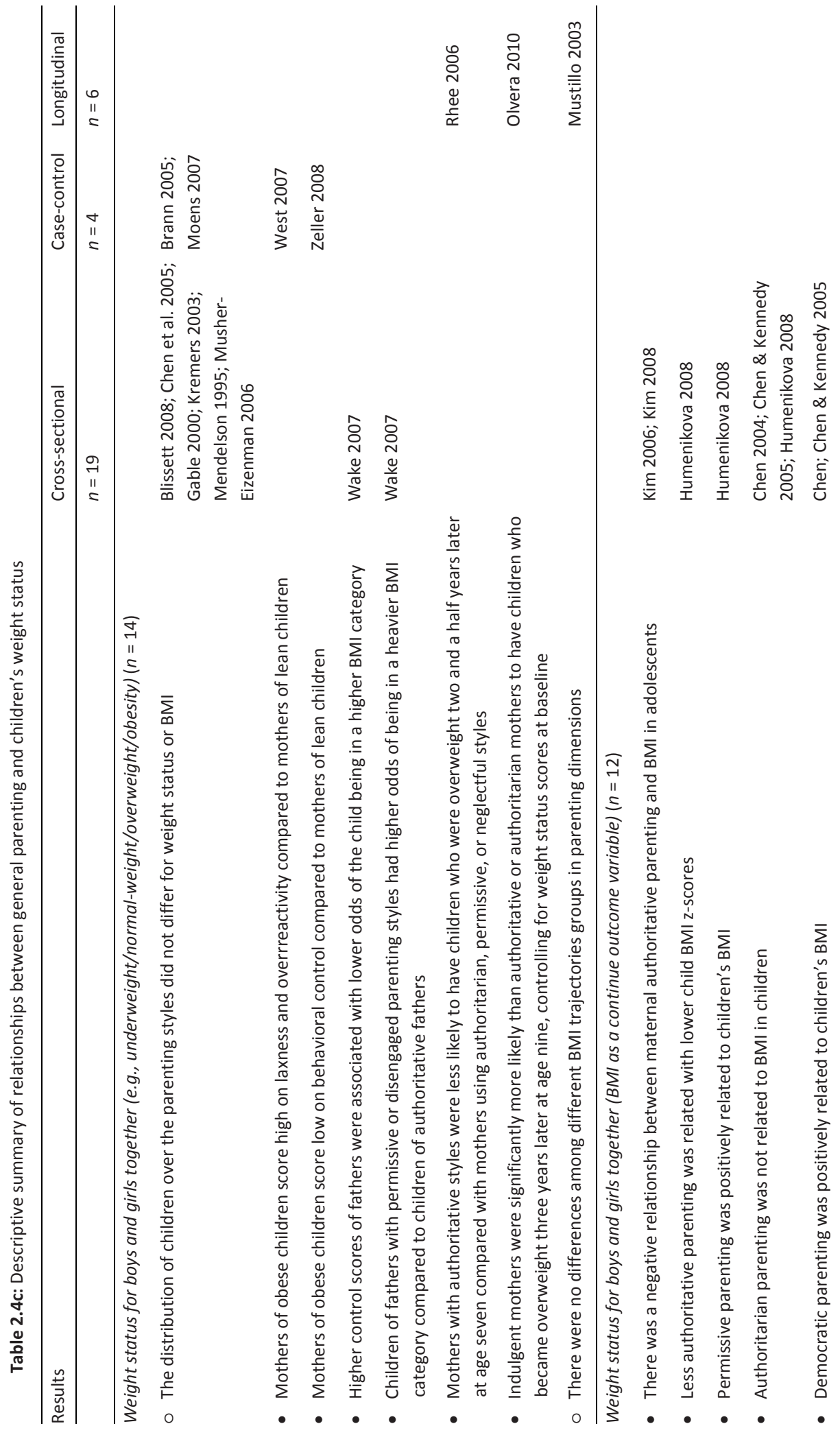


40 | CHAPTER 2

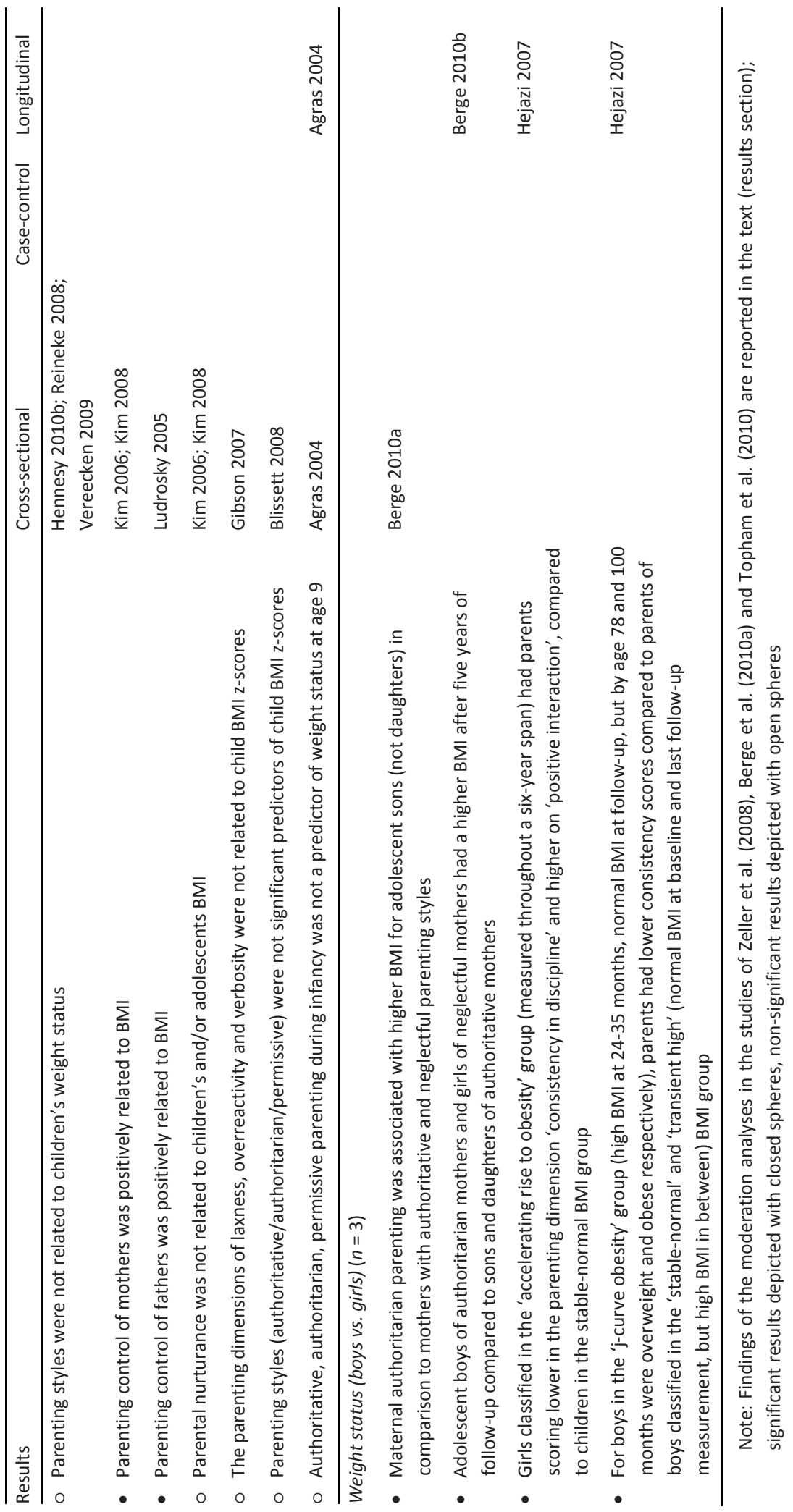




\section{Discussion}

The objective of the present review was to evaluate the existing literature examining the relationship between general parenting and children's weight-related outcomes. Some results were inconsistent. Generally, in many studies where a significant association with general parenting was found, children raised in authoritative homes were found to ate more healthily, to be more physically active, and to have lower BMI scores compared to children who were raised with a different style. An authoritative style is characterized by a family context of expressing warmth and emotional support, together with using clear, bidirectional communication (Maccoby \& Martin, 1983). These results are strengthened by prospective findings which inform us about the direction of causality. Five of the seven longitudinal studies show that general parenting at an early age has an impact on weight-related outcomes at a later date (Berge et al., 2010b; Hejazi, 2007; Lohaus et al., 2009; Olvera \& Power, 2010; Rhee et al., 2006). Furthermore, the findings of some moderation studies indicate that general parenting can have differential impact on children's weight status and related health behaviors, depending on characteristics of the child and the parents.

\section{Discrepancies in study results}

The parenting dimension 'nurturance' was typically positively related to overweight preventing behaviors of the child (e.g., high levels of physical activity) and negatively related to the child's obesity inducing behaviors (e.g., fat and caloric intake). For the dimension 'control' inconsistent findings were reported. Probably this is caused by different conceptualizations of controlling parenting. This dimension has been referred to as psychological control versus psychological autonomy, but also as lax control versus behavioral/firm control. Psychological control (opposite of psychological autonomy) was assessed in few of the included studies (Kim, 2006; Kim et al., 2008; Lohaus et al., 2009; Zeller et al., 2008). It is defined as 'parental behaviors (such as guiltinduction, love withdrawal or contingent love, instilling anxiety, and invalidation of the child's perspective) that are intrusive and manipulative of children's thoughts, feelings, and attachments to parents' (Barber \& Harmon, 2002). This construct was related to negative behavioral outcomes (e.g., high intake of calories and fat) (Kim, 2006; Kim et al., 2008). Although this type of control by mothers was related to BMI z-scores of the children in the study by Kim (2006; 2008), there was no difference between obese and non-overweight youth in reported psychological control in the study of Zeller and others (2008). Using another operationalization, Lohaus et al. (2009) created the four prototypes of parenting based on three dimensions of parental behavior; besides 'warmth' and 'behavioral control' incorporating 'psychological pressure' in the classification of parenting typologies. Authoritative parenting was characterized by high lev- 
els of behavioral control and low levels of psychological pressure and related to the most positive health outcomes (Topham et al., 2010). Very closely related to the construct of psychological control is the restrictiveness dimension assessed in the Child Rearing Practices Report (Block, 1981). Ludrosky (2005) found that paternal restrictiveness was positively related to children's BMI and caloric intake. Lax control, defined as inconsistent discipline (chaos) has been assessed in some studies (Gibson et al., 2007; Hejazi, 2007; Mustillo et al., 2003; West, 2007; Zeller et al., 2008), usually indicating higher scores on inconsistent discipline of parents in the obese group compared to parents of non-overweight children (West, 2007; Zeller et al., 2008) and for parents of children with rapid excessive weight gain (Hejazi, 2007).

Besides the repeated findings for the negative influence of psychological and lax control on children's health behaviors, positive effects were reported for behavioral control (e.g., Lohaus et al., 2009; Wake et al., 2007; Zeller et al., 2008). However, controversies exist regarding the optimal level of control. For instance, Van der Horst et al. (2007) found evidence that general parenting modified the relationship between restrictive feeding practices and adolescents sugar-sweetened beverage consumption: restrictive feeding was associated with lower intake of sugar-sweetened beverages when parents were highly involved, but moderately controlling (Van der Horst et al., 2007). Such results could indicate that both low control and very strict, overcontrolling types of parenting are counterproductive, indicating a U-shaped relationship between parental control and child weight. Despite the availability of a large number of parenting instruments, measurement tools assessing the apparent broad range of controlling dimensions are currently lacking. Therefore, one should be very cautious in comparing and interpreting the study results of the included studies.

Differences in conceptualization of parenting constructs may also explain other inconsistent findings regarding the relationship between general parenting and children's weight-related outcomes. In some studies instruments were used that can assess parenting style without crossing scores on separate parenting dimensions (Agras et al., 2004; Blissett \& Haycraft, 2008; Brann \& Skinner, 2005; Chen \& Kennedy, 2004; Chen \& Kennedy, 2005; Chen et al., 2005; Chen et al., 2008; Gable \& Lutz, 2000; Humenikova \& Gates, 2008; Lytle et al., 2003; Mendelson et al., 1995; MusherEizenman \& Holub, 2006; Reineke, 2008; Schmitz et al., 2002; Topham et al., 2010). In other studies parenting styles were constructed based on the scores on separate parenting dimensions (Berge et al., 2010a; Berge et al., 2010b; De Bourdeaudhuij et al., 2009; Hennessy et al., 2010; Kim, 2006; Kim et al., 2008; Kremers et al., 2003; Lohaus et al., 2009; Olvera \& Power, 2010; Pearson et al., 2010; Rhee et al., 2006; Vereecken et al., 2009; Wake et al., 2007). Typically, parenting styles were categorized into four prototypes (authoritative, authoritarian, indulgent/permissive, and neglectful/uninvolved) based on splits of two parenting dimensions (e.g., nurturance/warmth and 
amount of control, involvement and strictness, sensitive to child's need and expectations for self-control, responsiveness and demandingness). In six of these studies (De Bourdeaudhuij et al., 2009; Hennessy et al., 2010; Olvera \& Power, 2010; Pearson et al., 2010; Rhee et al., 2006; Vereecken et al., 2009) this categorization was based on median splits of both dimensions, a method which has to be applied carefully. For instance, if all parents of a study sample score very low on authoritarian control, using median splits, it is possible that parents scoring in the higher end on this dimension are classified as being authoritarian, whereas these parents would not be classified as being authoritarian in a different sample. Furthermore, when using median splits to define parenting styles, it is not possible to compare the study results with other studies, since scores on various parenting dimensions may differ across samples'.

Only in one study the categorization into prototypes of parenting was based on three dimensions (warmth, control, psychological pressure) (Lohaus et al., 2009). Of 13 studies that assessed separate parenting dimensions, ten studies only reported on relationships between parenting styles and children's weight-related outcomes (Berge et al., 2010a; Berge et al., 2010b; De Bourdeaudhuij et al., 2009; Hennessy et al., 2010; Kremers et al., 2003; Lohaus et al., 2009; Olvera \& Power, 2010; Pearson et al., 2010; Rhee et al., 2006; Vereecken et al., 2009), rather than also assessing relationships with separate parenting dimensions. In doing so, important information could be lost. For instance, parents scoring moderately on both dimensions could be falsely categorized into a parenting style. Additionally, some studies (e.g., Gibson et al., 2007; West, 2007; Zeller et al., 2008) assessed parenting constructs as being bipolar (i.e., parents scoring high on a parenting construct are expected to score low on its opposite). Recently, however, Skinner et al. (1953) provided empirical support for the multidimensionality of parenting constructs rather than treating those constructs as being bipolar; parents scoring high on one parenting dimension (e.g., acceptance) do not necessarily score low on its conceptual opposite (i.e., rejection). Future researchers should take into account this multidimensionality.

Study results could differ according to the person completing the parenting instruments. The current review revealed that this could be done by both parents separately, one of the parents, or the child. Especially parental self-reporting could be a limitation of some of the studies, which may be biased because of social desirability. This may also decrease comparability with other studies which measure general parenting constructs via children or adolescents, although these constructs are also measured via questionnaires and thus subjective measures. Differences in the relationship between general parenting and children's weight-related outcomes were found for mothers and fathers (e.g., Kim, 2006; Kim et al., 2008; Lohaus et al., 2009; Ludrosky, 2005; Lytle et al., 2003; Schmitz et al., 2002). It also seems that differences among the samples (e.g., sample size, child gender, age and ethnicity) may explain 
some of the inconsistencies. Study findings should be interpreted with caution as the variables (i.e., general parenting and children's weight-related outcomes) are all measured in different ways. However, most outcome variables were measured with validated instruments. It was not possible to calculate power and effect sizes of each study because of the heterogeneity of measurements across the studies and lacking information (e.g., regarding distribution of independent and outcome variables) in many studies.

Furthermore, several variables (e.g., socio-economic status, maternal depression, child temperament) have been found to interact with certain parenting styles or dimensions in predicting children's weight status. Two studies examined the role of general parenting as a contextual factor that can influence the effectiveness of foodrelated parenting practices in predicting children's dietary intake behaviors (MusherEizenman \& Holub, 2006; Van der Horst et al., 2007). In line with this, we came across studies assessing parent-child interactions in relation to children's weight status in a broader context, e.g., quality of a child's environment (Beck \& Terry, 1985; Lissau \& Sorensen, 1994; Strauss \& Knight, 1999; Turner, Rose, \& Cooper, 2005; Zeller et al., 2007), parental stress (Stenhammar et al., 2010), attachment (Stenhammar et al., 2010; Trombini et al., 2003). Since we were specifically interested in parenting style (dimensions), these studies were not included in the present review.

\section{Recommendations for future research}

Additional research is needed to further study the influence of mediating and moderating factors influencing the general parenting - child weight relationship, preferably employing a longitudinal design with more extended follow-up periods to establish causation. The proposed conceptual model (see Figure 2.1) could be used in order to guide determinant research, thereby enabling better understanding of the general parenting - child weight relationship. There is a need to conduct determinant studies using diverse ethnic samples and age groups. Moreover, larger samples of fathers should be included to allow for comparisons between mothers and fathers.

Increasingly, intervention studies address general parenting in the prevention and treatment of childhood overweight and obesity (Gerards, Sleddens, Dagnelie, De Vries, \& Kremers, 2011). We recommend intervention developers to increase their attention to the family context as it is an important factor influencing outcomes of overweight and obesity interventions for children. The primary goal of this type of interventions should be to create authoritative environments characterized by parental encouragement of instrumental competence in children by helping them in balancing otheroriented, rule-following tendencies with individualistic, autonomous active thinking (Baumrind, 1978; Newman, Harrison, Dashiff, \& Davies, 2008; Spera, 2005). 


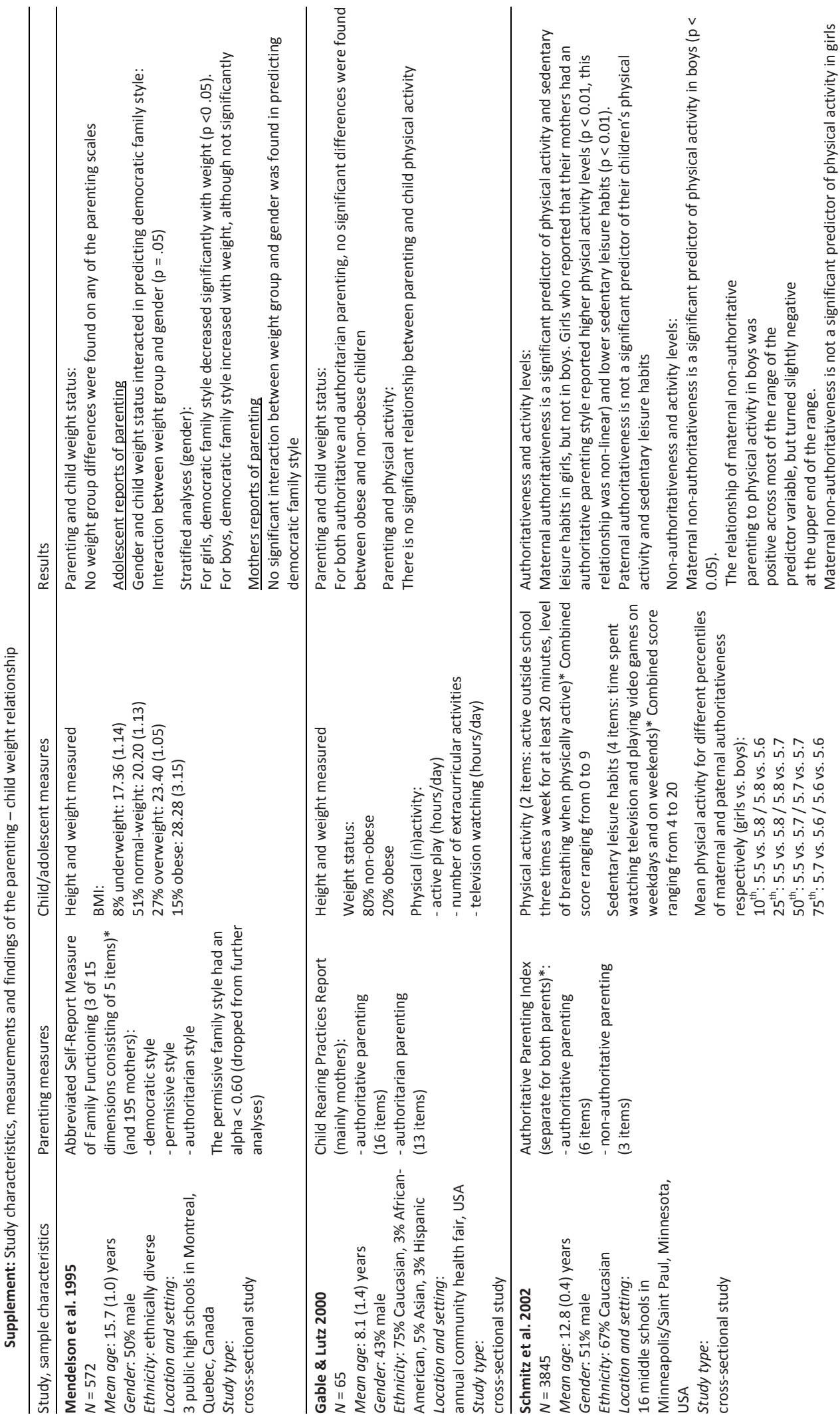



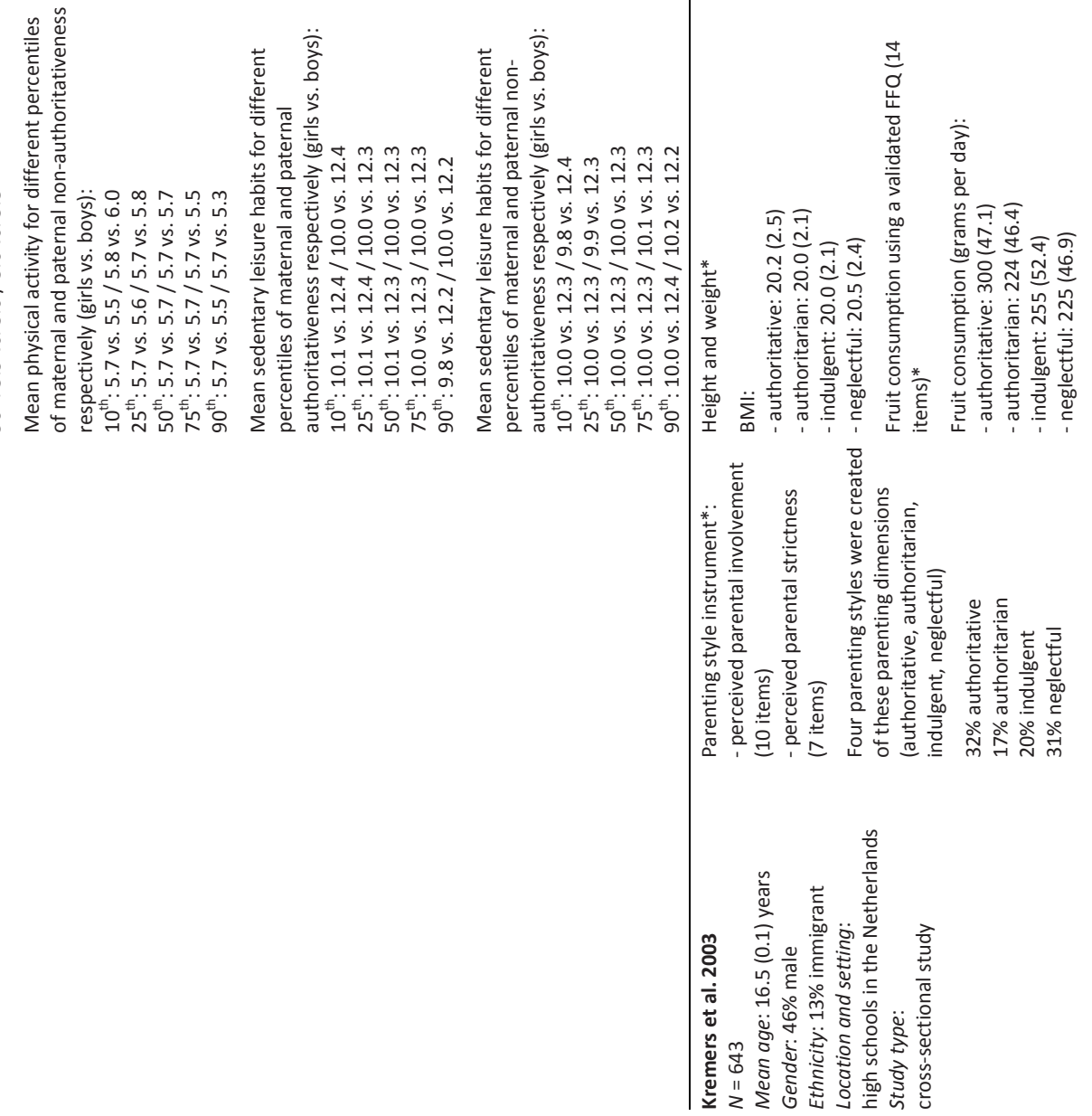


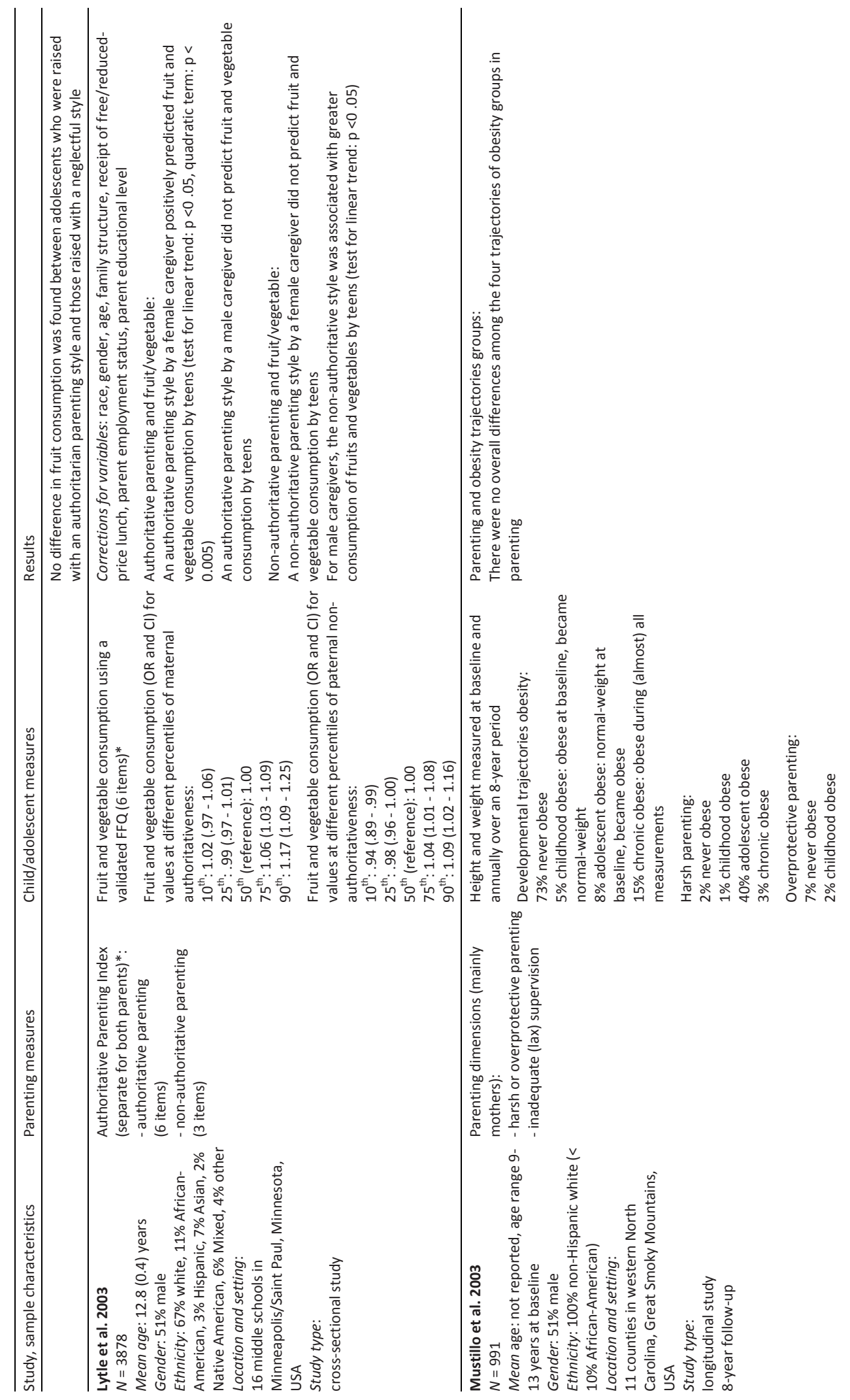




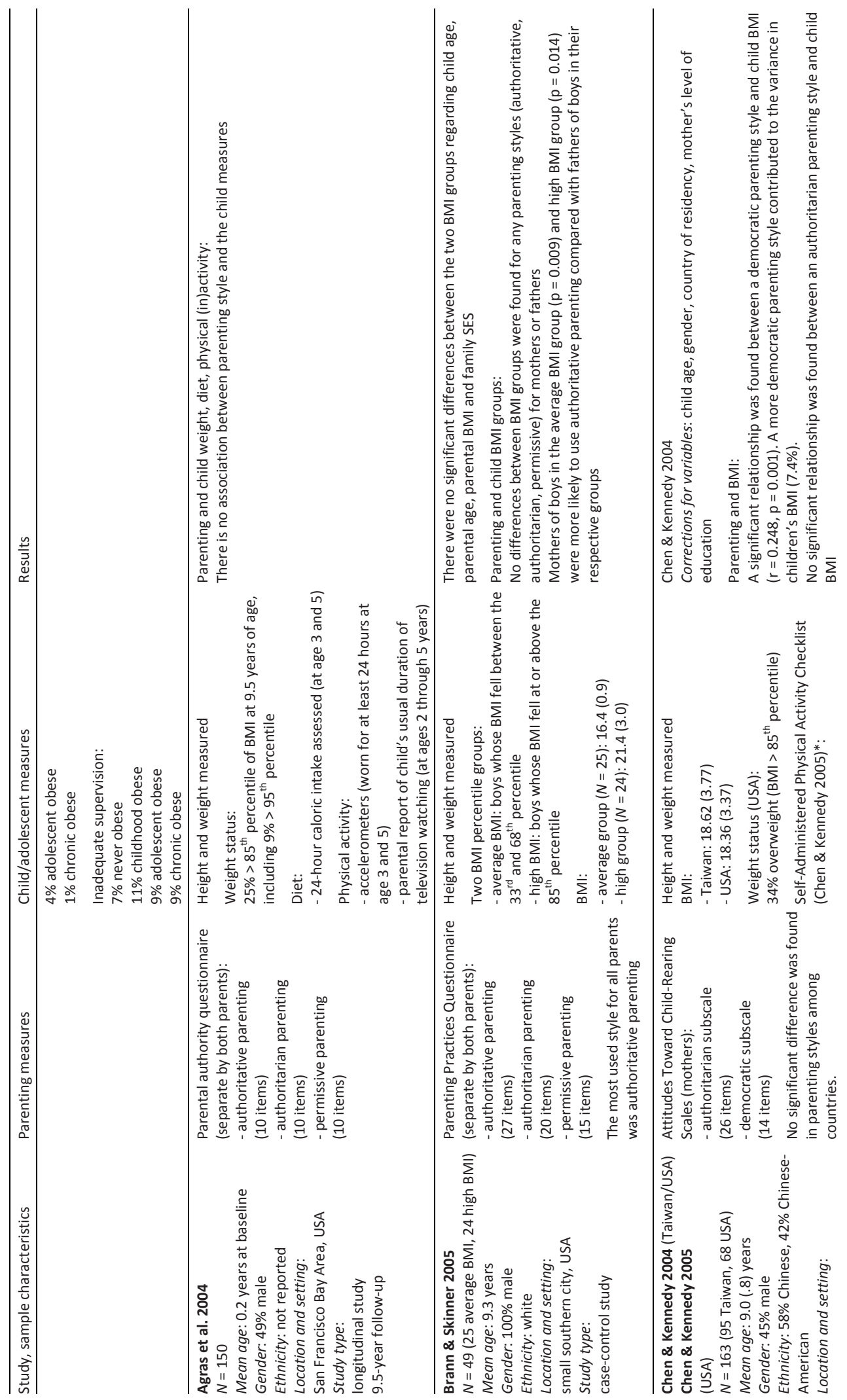




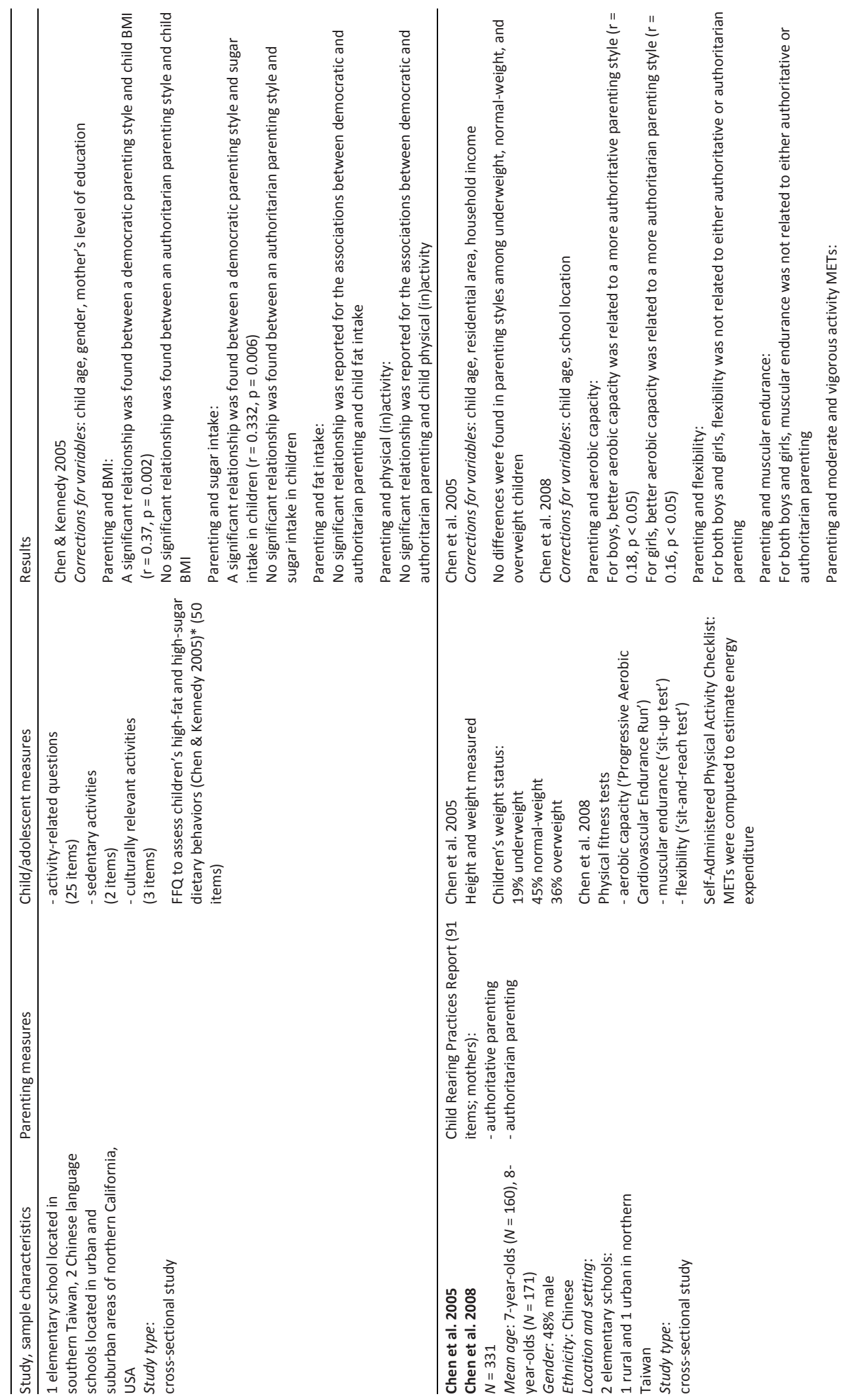




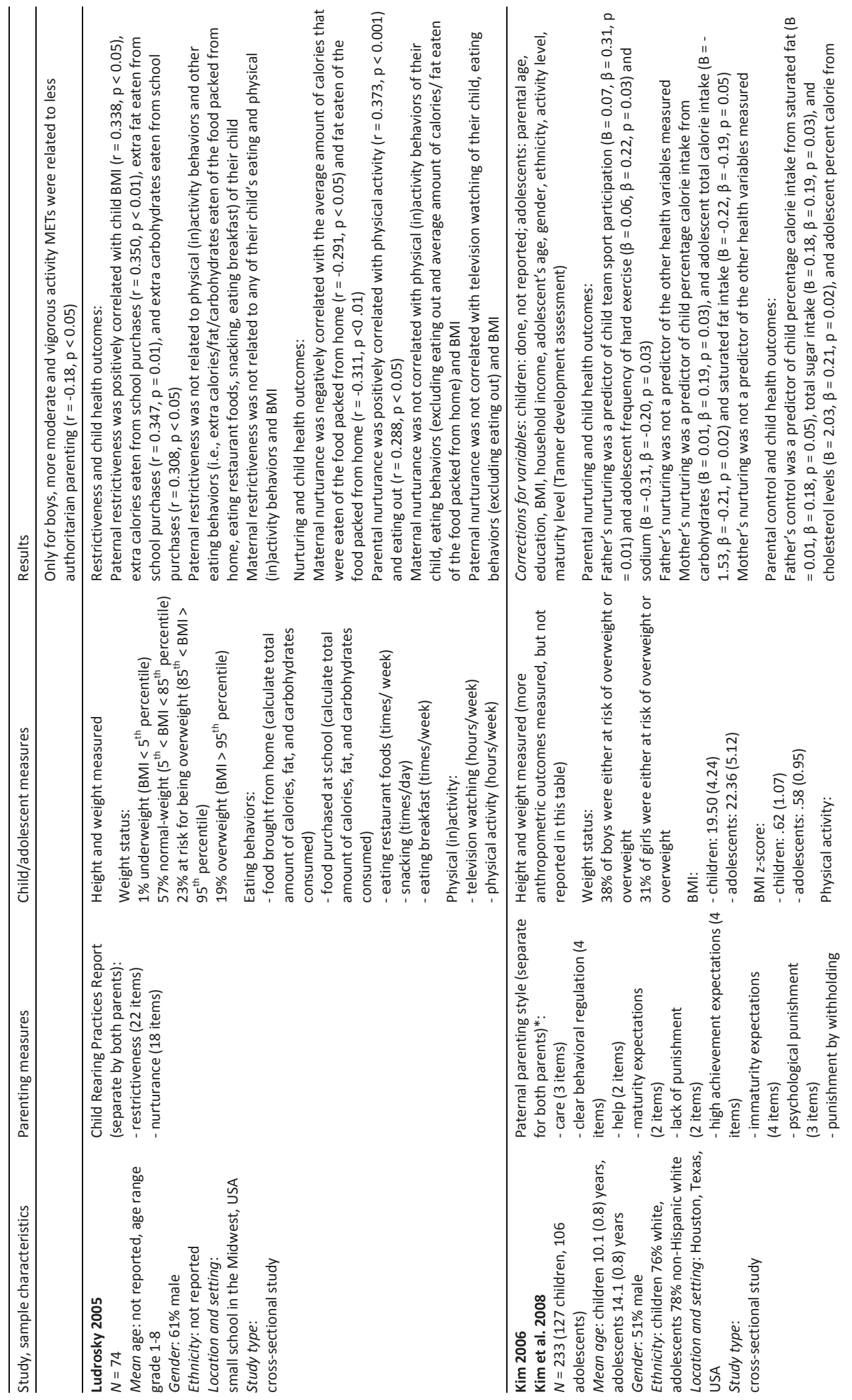




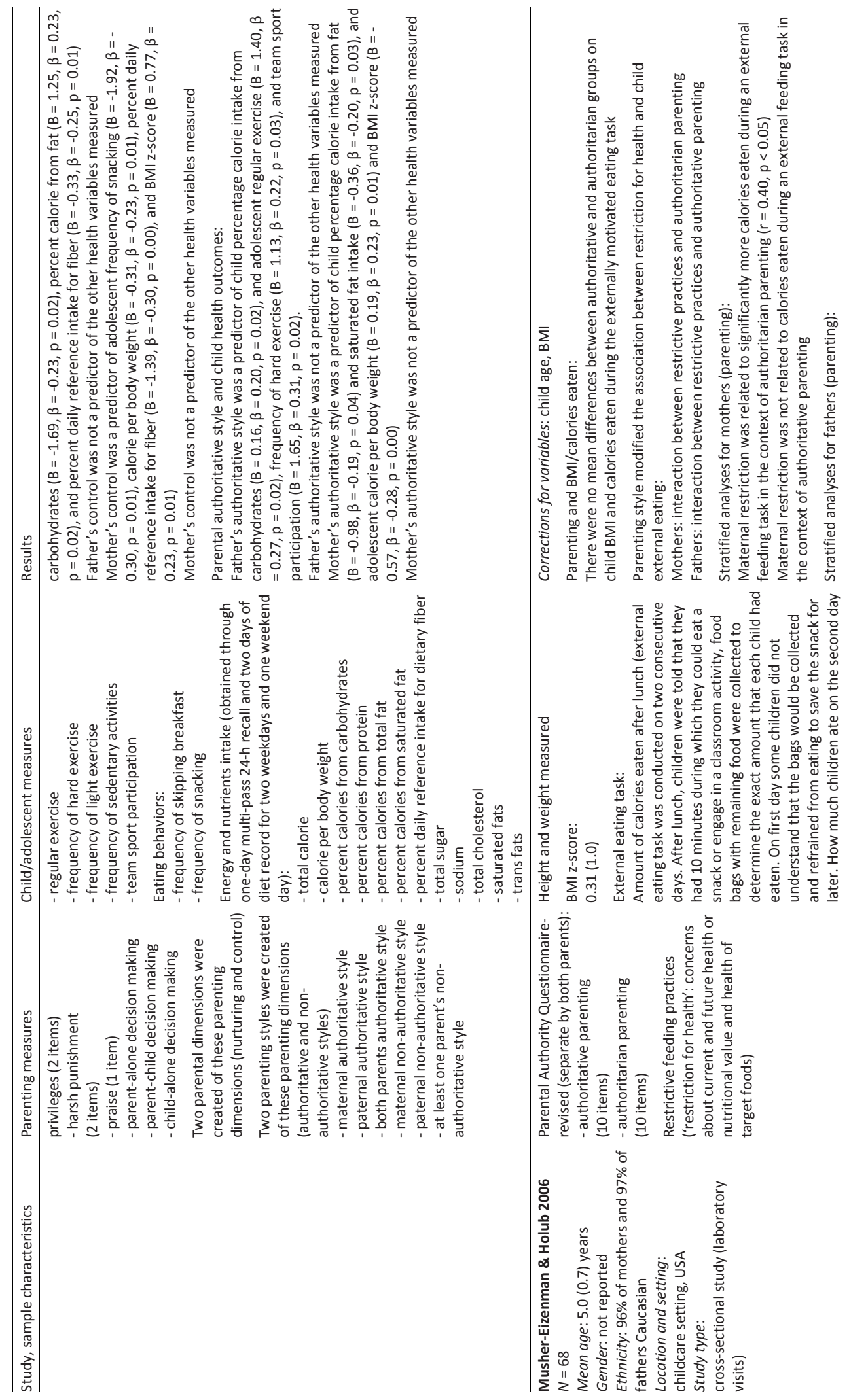




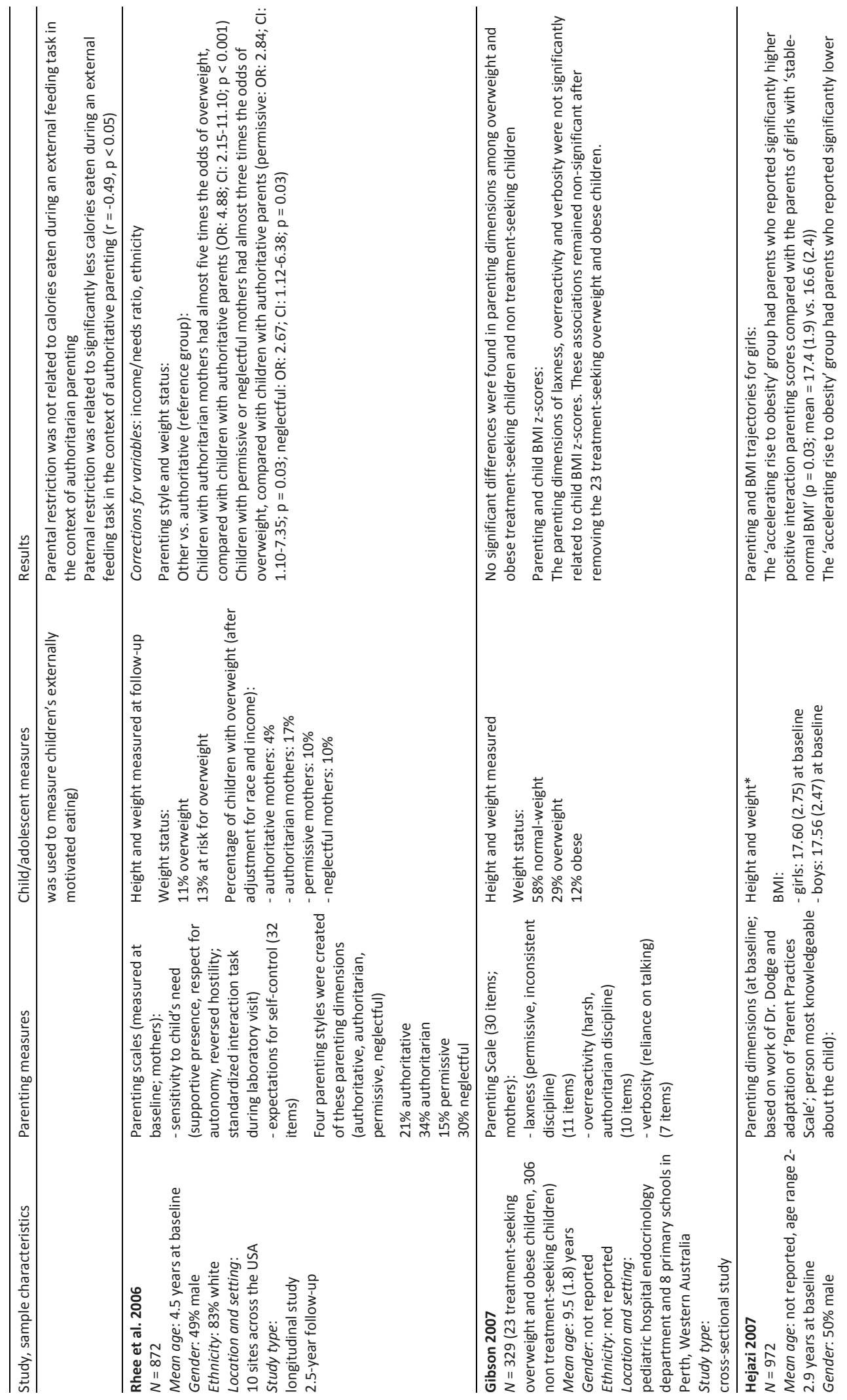




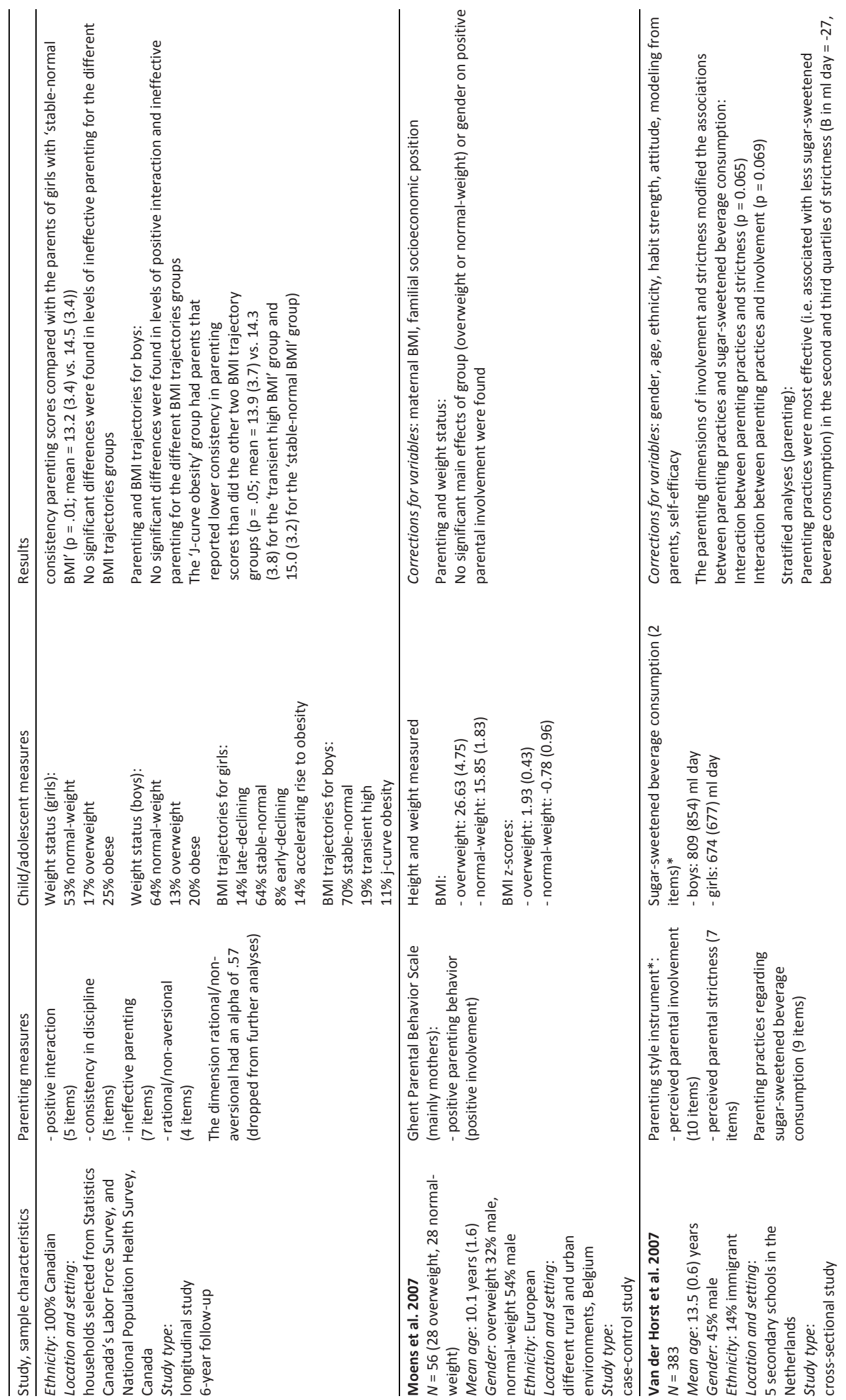


54 | CHAPTER 2

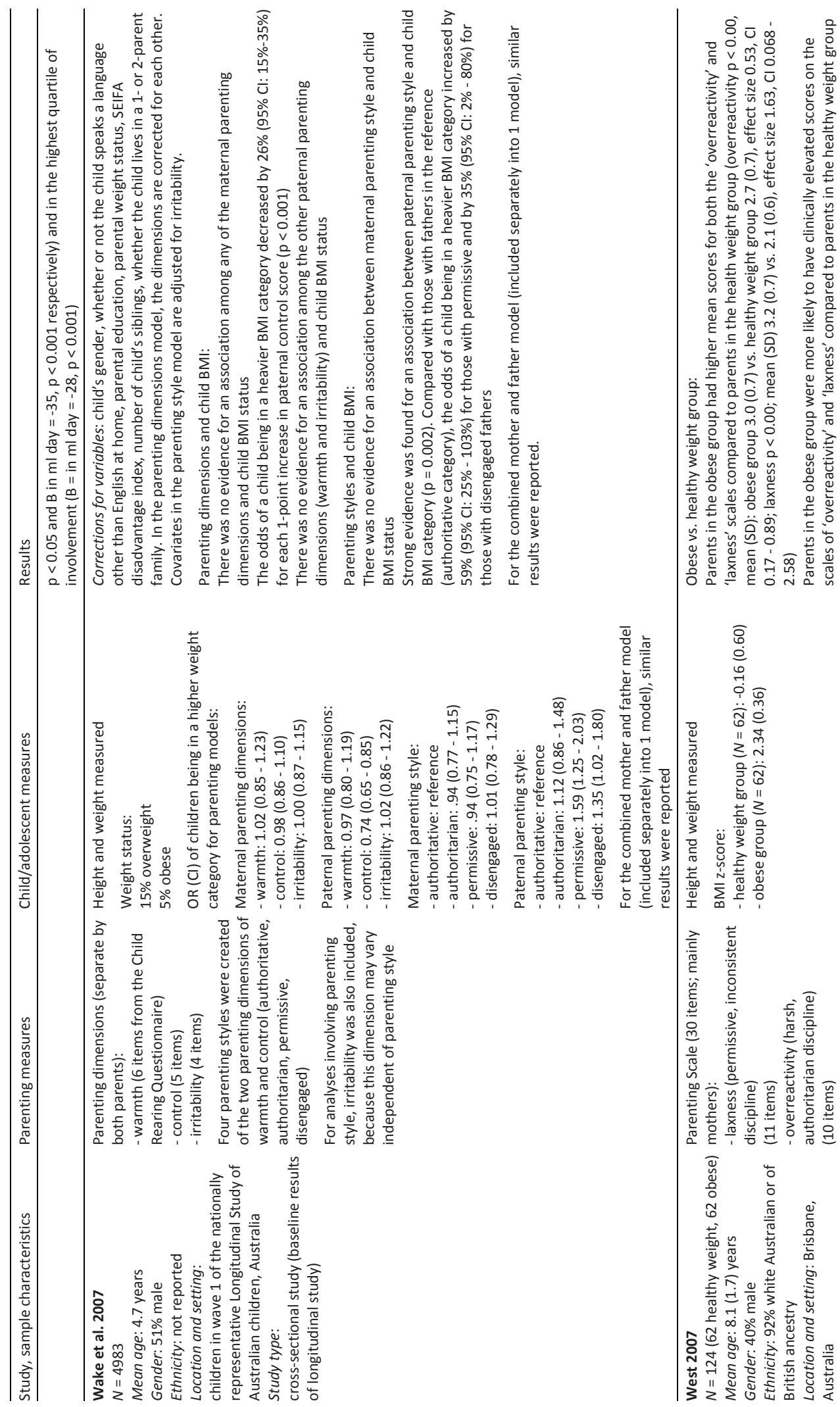




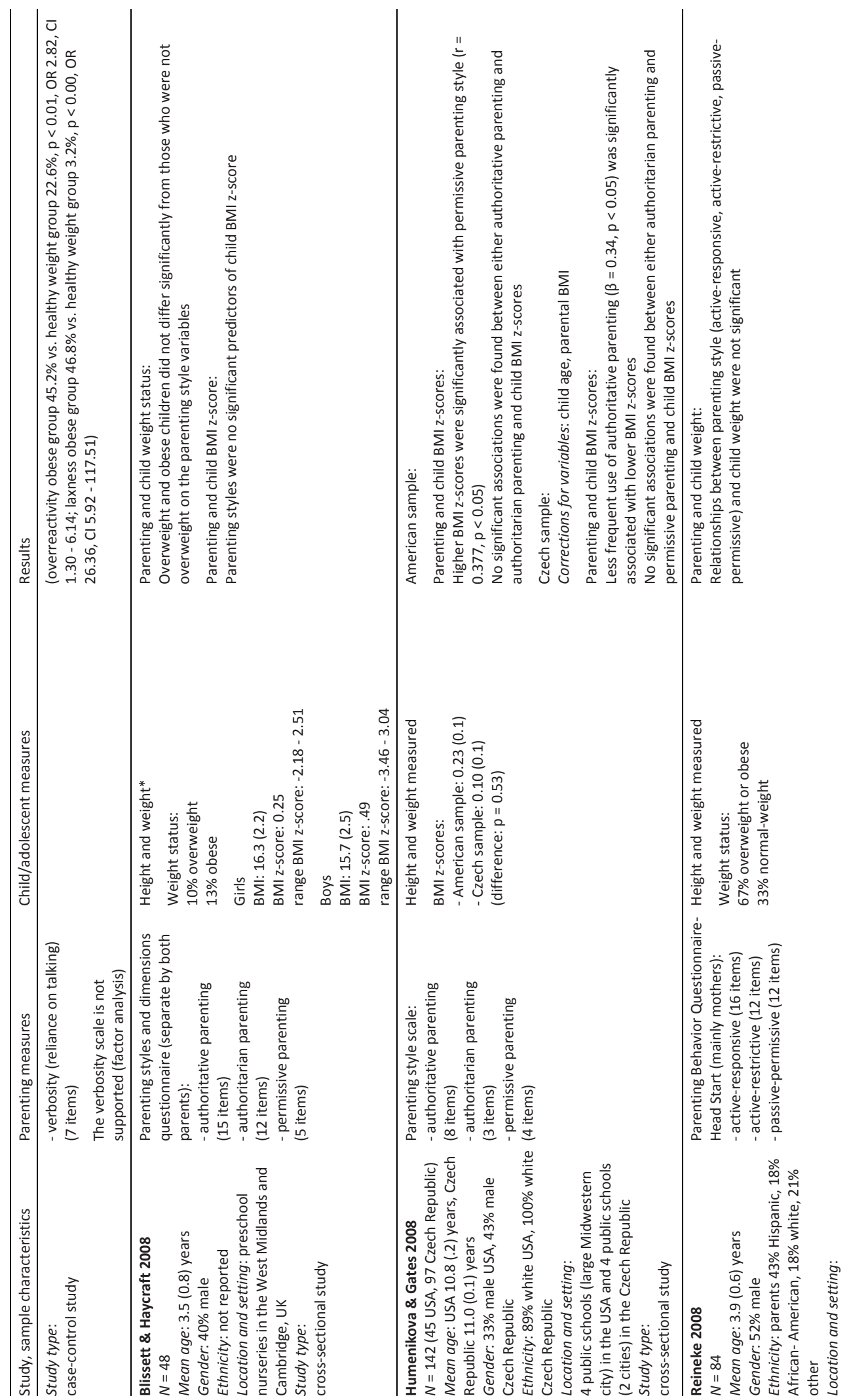




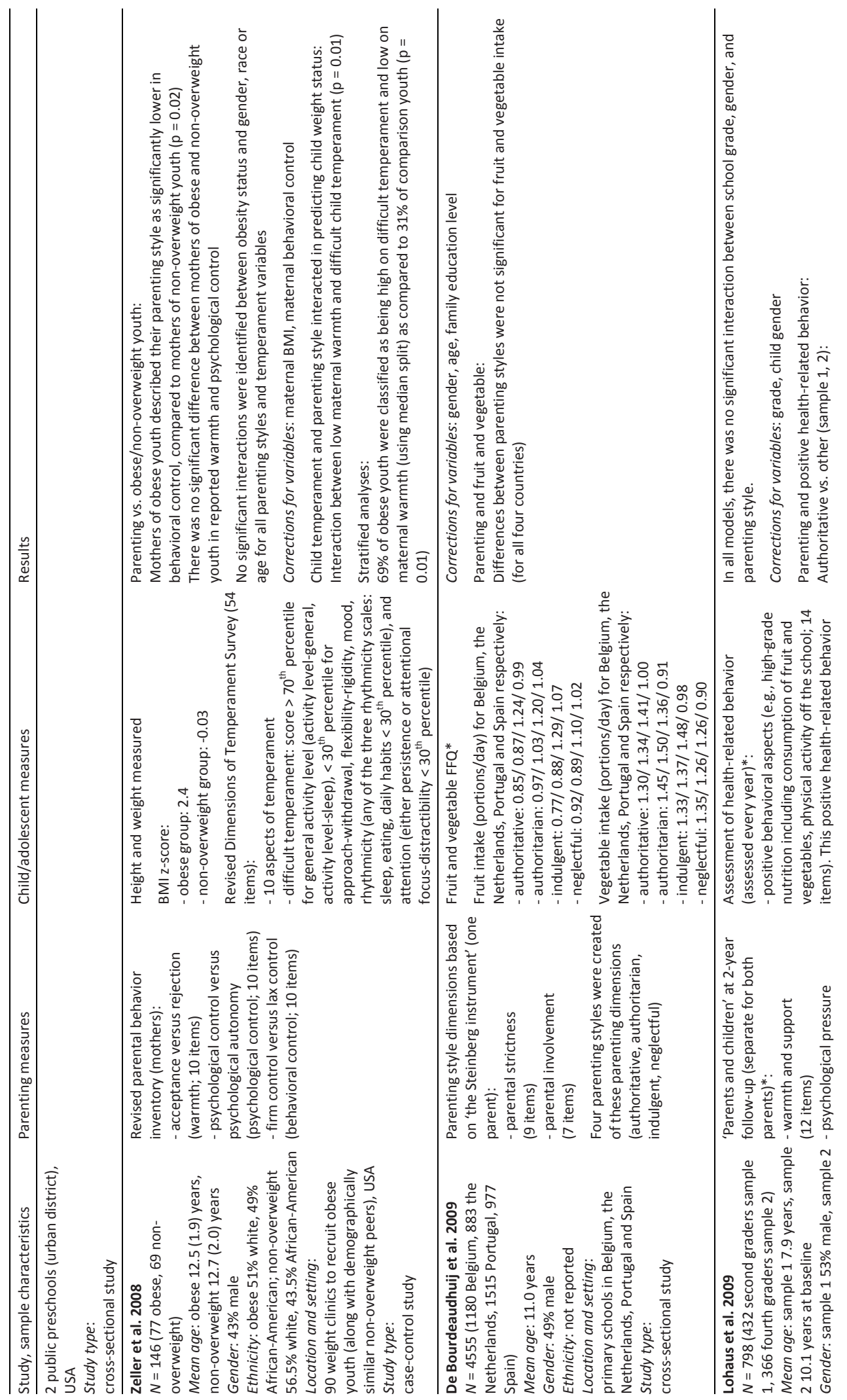




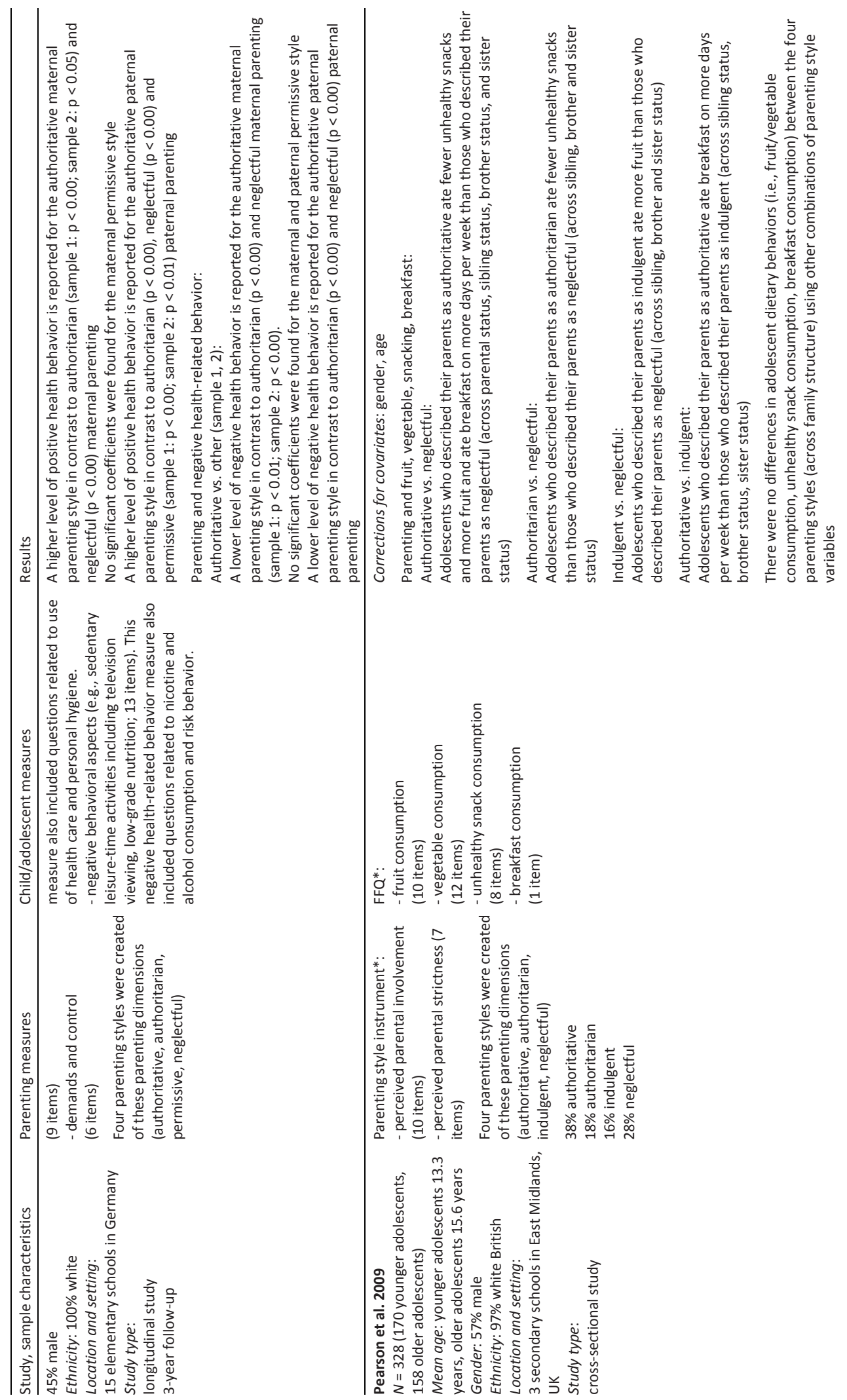


58 | CHAPTER 2

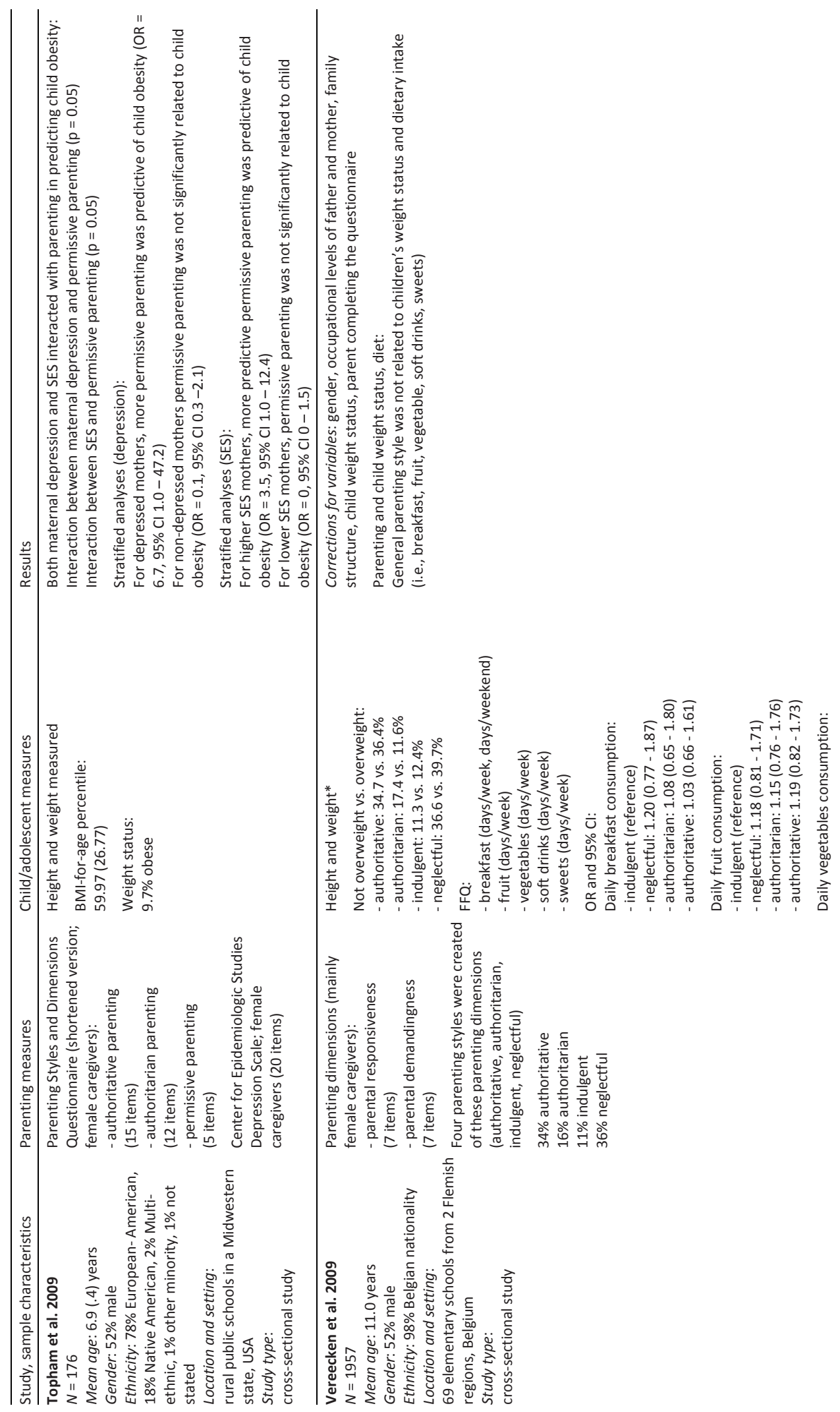




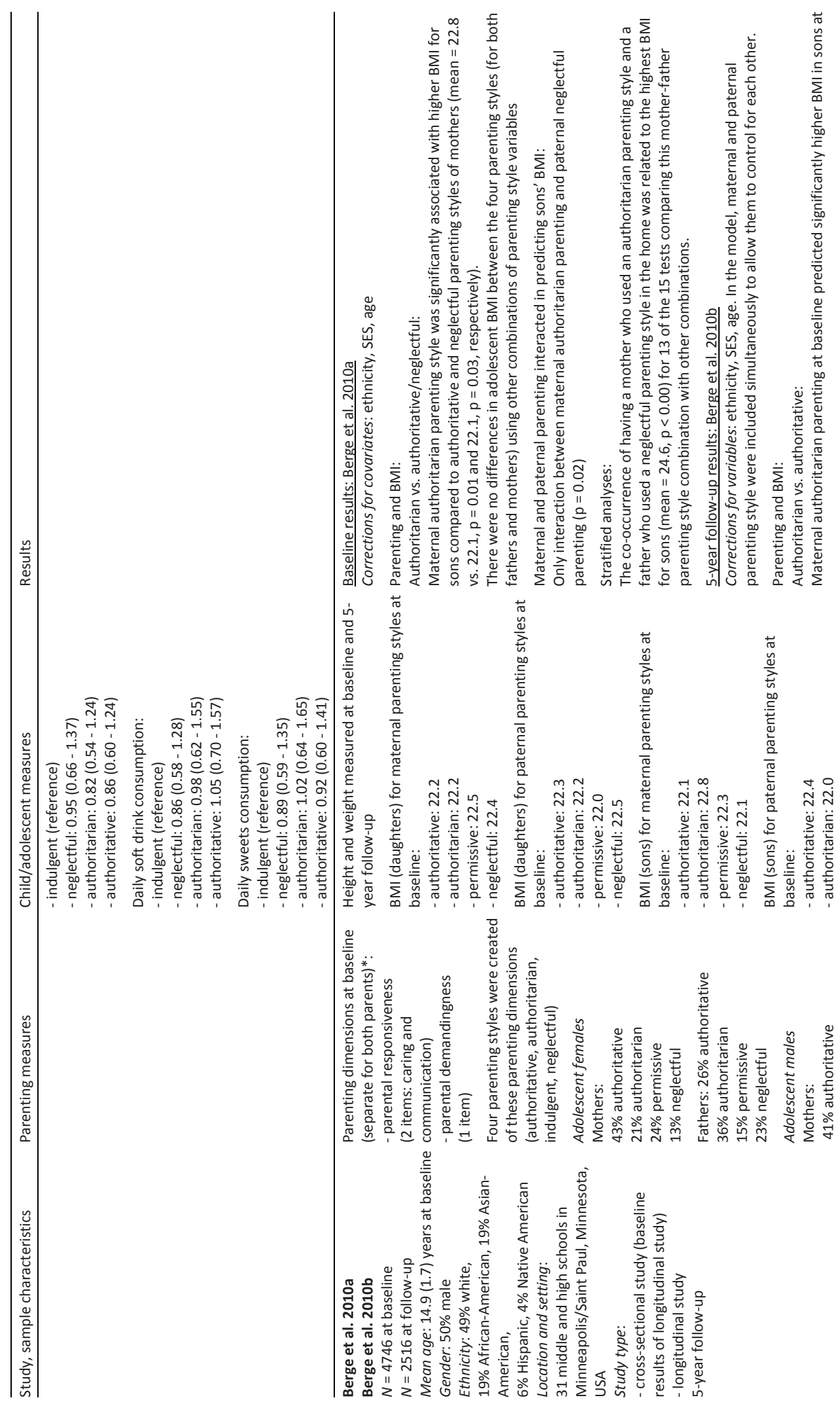




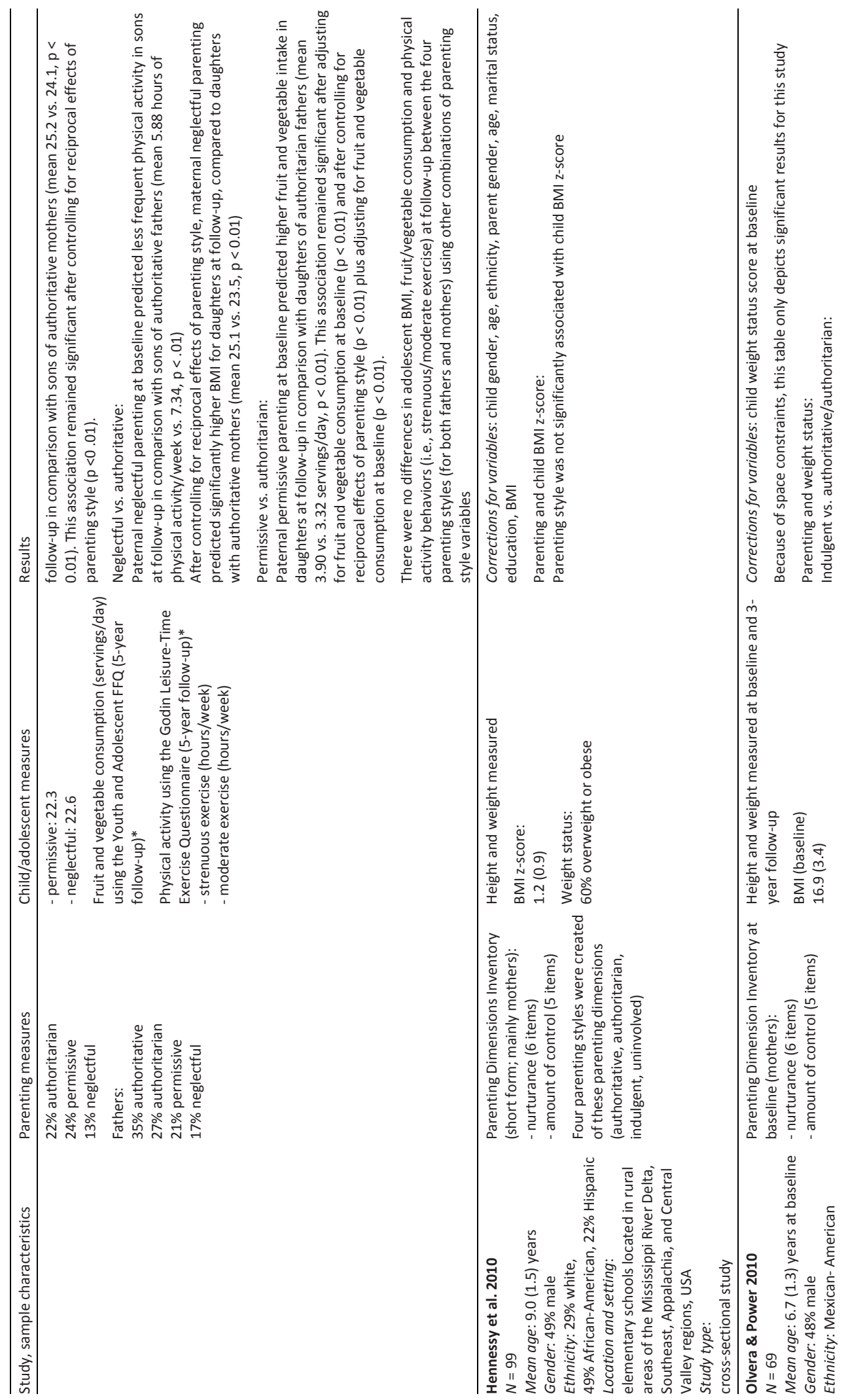




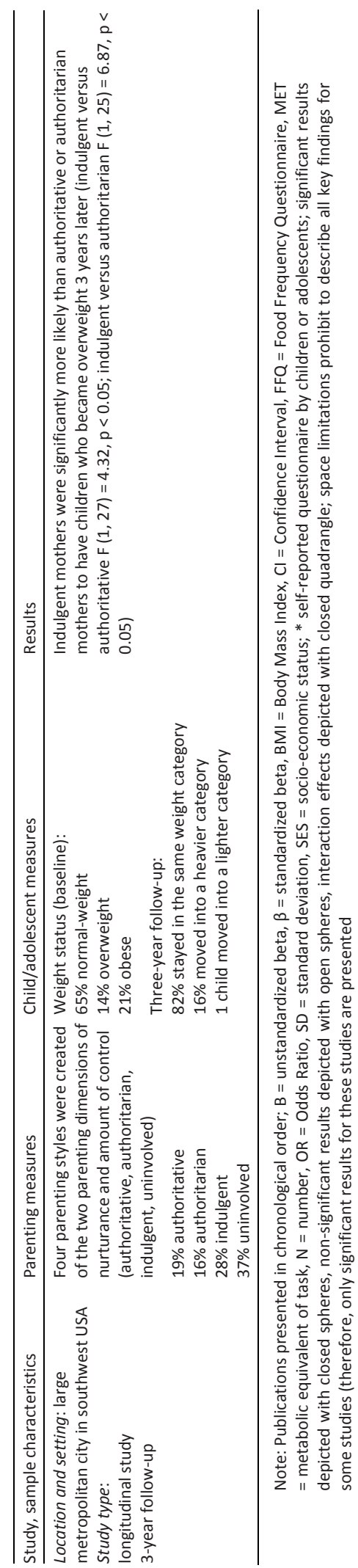





\section{CHAPTER 3}

\section{Interventions addressing general parenting to prevent or treat childhood obesity}

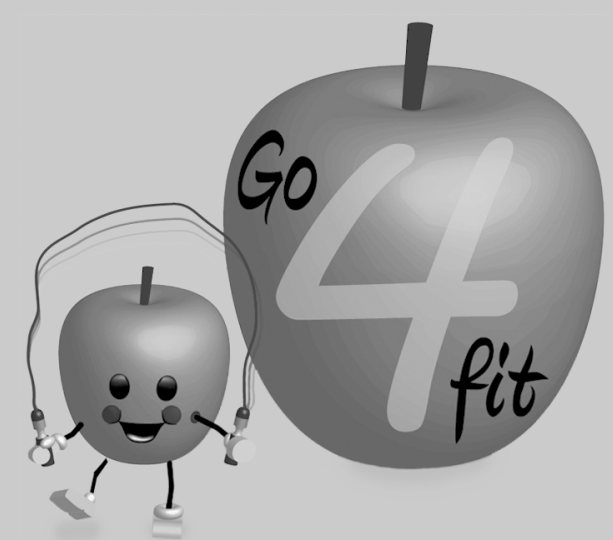

Published as: Gerards, S.M.P.L., Sleddens, E.F.C, Dagnelie, P.C., De Vries, N.K. and Kremers, S.P.J. (2011). Interventions addressing general parenting to prevent or treat childhood obesity. International Journal of Pediatric Obesity, 6: e28-e45 


\begin{abstract}
Observational studies increasingly emphasize the impact of general parenting on the development of childhood overweight and obesity. The aim of the current literature review was to provide an overview of interventions addressing general parenting in order to prevent or treat childhood obesity. Electronic literature databases were systematically searched for relevant studies. Seven studies were eligible for inclusion. The studies described four different general parenting programs, which were supplemented with lifestyle components (i.e., physical activity and nutrition). All studies showed significant small to moderate intervention effects on at least one weight-related outcome measure. The current review shows that despite the emerging observational evidence for the role of parenting in children's weight-related outcomes, few interventions have been developed that address general parenting in the prevention of childhood obesity. These interventions provide evidence that the promotion of authoritative parenting is an effective strategy for the prevention and management of childhood obesity.
\end{abstract}




\section{Introduction}

Overweight and obesity in children is a significant public health problem (Wang \& Lobstein, 2006; WHO, 2006). The current prevalence of overweight and obesity varies considerably across parts of the world, with North America, Europe and parts of the Western Pacific having the highest prevalence of overweight among children (approximately 20-30\%) (Wang \& Lobstein, 2006). Increasingly, children become overweight at a relatively young age. Being overweight as a child increases the risk of becoming an overweight adult, compared to normal-weight children (Freedman et al., 2005; Magarey, Daniels, Boulton, \& Cockington, 2003; Singh et al., 2008). Obesity in childhood is also associated with health consequences like cardiovascular diseases and type 2 diabetes, and social consequences such as teasing and discrimination (Must \& Strauss, 1999; Strauss, 2000).

A large number of interventions to prevent or treat overweight in childhood have been developed, which have been extensively described in various systematic reviews (Bluford, Sherry, \& Scanlon, 2007; Campbell \& Hesketh, 2007; Doak, Visscher, Renders, \& Seidell, 2006; Oude Luttikhuis et al., 2009; Saunders, 2007; Summerbell et al., 2005). These reviews show that the majority of the interventions focus on changing so-called energy balance-related behaviors, that is, improving children's dietary intake and increasing their levels of physical activity (Kremers et al., 2005). However, there is still debate on the best way to design an intervention to achieve maximal and sustainable effects on child outcomes (Summerbell et al., 2005).

Intervention research has increasingly highlighted the importance of parents and family involvement in child obesity treatment and prevention (Kitzmann \& Beech, 2006; Nowicka \& Flodmark, 2008; Young, Northern, Lister, Drummond, \& O'Brien, 2007). Parents determine their child's lifestyle to a large extent, especially in the early years of life, and several intervention studies have demonstrated that involving the family in the treatment of childhood overweight is an effective approach. For example, Epstein and colleagues showed that including parents as active participants in habit change and weight loss was effective in terms of weight control among children at fiveyear follow-up (Epstein, McCurley, Wing, \& Valoski, 1990); these effects were maintained over extended periods from childhood through adolescence and adulthood (Epstein, Valoski, Wing, \& McCurley, 1990, 1994b). Golan et al. (2006) showed that targeting parents as exclusive moderators resulted in greater reduction in children's percentage overweight than targeting both parents and children or targeting children alone. Similarly, prevention studies have shown that parents can be supported in achieving effective behavioral changes that seem likely to reduce the degree of overweight in their children (Campbell \& Hesketh, 2007). 
A distinction between specific and general family-based intervention goals has been made in the existing literature (Kitzmann \& Beech, 2006). A specific intervention focus involves parents in changing specific child behaviors related to eating and exercise, i.e. changing specific parenting practices. A general intervention, aims at changing the broader family context or family functioning (Kitzmann \& Beech, 2006). One important dimension of the family context concerns 'general parenting' or 'parenting styles', which is the focus of the current paper. It describes parent-child interactions across a wide range of situations, and can be regarded as the context of behavior-specific parenting (Darling \& Steinberg, 1993). Parenting styles are often rated using two dimensions: an index of parental responsiveness (warmth, acceptance or involvement) and an index of parental demandingness (control or strictness) (Maccoby \& Martin, 1983). The combination of these dimensions results in a fourfold classification of parenting: authoritative, authoritarian, indulgent (similar to permissive), and neglectful (similar to uninvolved).

While originally (and successfully) applied in the prediction of childhood problem behaviors, to date multiple observational studies have also provided evidence for the impact of general parenting on children's weight status and related health behaviors (Sleddens, Gerards, Thijs, De Vries, \& Kremers, 2011). Such observational studies have indicated the potential of general interventions addressing these general parenting skills in attempts to prevent or treat childhood obesity. Although some researchers already underlined the importance of targeting the broader family context in the prevention or treatment of childhood obesity (Kitzmann \& Beech, 2006; Kitzmann et al., 2008), to date no reviews have focused exclusively on interventions that incorporated general parenting. The aim of the current review was therefore to provide an overview of interventions which address general parenting in order to prevent or treat obesity in youth (0-18 years).

\section{Methods}

Studies that were eligible for the present systematic review were searched for in the computerized databases Pubmed, PsycINFO and Scopus, using combinations of the following keywords: parents, parenting, child, infant, overweight, obesity, weight gain, intervention, and prevention. We included dissertations and studies published in peerreviewed journals until February 2010.

Studies were first assessed on their title and then on their abstract, by two reviewers (S.G. and E.S.) independently of each other. To be included in the present study, intervention studies had to meet the following inclusion criteria:

1. The intervention study had to focus on children or adolescents (aged 0-18). 
2. The primary aim of the intervention had to be the prevention or treatment of overweight or obesity.

3. The study had to describe intervention components addressing general parenting.

4. The study had to have used anthropometric measurements at baseline and follow-up to describe the effectiveness of the intervention.

5. The article had to be in English.

Studies which aimed to change to the children's broader environment, e.g., the emotional climate of the family were excluded from the present review.

In total, 1057 papers were initially identified in Pubmed, Psychlnfo and Scopus using the selected keywords. These were all screened on title, leading to 118 eligible hits. Of these, 55 were eliminated based on abstract evaluation. The remaining 63 papers were retrieved for reading the full-text. This resulted in four studies which were considered eligible for inclusion. Major reasons for studies not being included in the current review were: no intervention studies $(n=757)$, interventions not aimed at treatment or prevention of childhood overweight $(n=133)$, interventions not targeting children or not including parents $(n=69)$, no intervention components on general parenting ( $n=69$ ), no intervention effects reported $(n=14)$, and paper not in English available $(n=15)$. After the electronic literature search, we applied reference tracking, which led to three additional references, leading to seven eligible studies.

\section{Data extraction and analysis}

The data extraction process of the included studies consisted of three steps. First, we described the general characteristics of the studies: location, inclusion and exclusion criteria of the study participants, design, treatment groups, follow-up, drop-out rates, and characteristics of the study sample. Second, the intervention used in the included studies was outlined by describing the duration, the target group, the components of the intervention targeting general parenting, and the components of the intervention addressing physical (in)activity and nutrition. The final part of the qualitative data extraction process regarded the results of the interventions, including the anthropometric measures of overweight. If available, measures of physical activity (or inactivity) and nutrition and parenting measures were also recorded. These data were abstracted by the first author (S.G.) and checked by the second author (E.S.).

In addition, effect sizes (Cohen's d) were calculated for tests of differential change in weight measures across the intervention and control conditions. Cohen's $d$ is calculated by dividing weight changes by the pooled standard deviation of the baseline weight of the study population (Cohen, 1992). In the case of multiple intervention groups, the effect sizes of all interventions were assessed. In studies without an appropriate control group, Cohen's d was calculated by dividing the mean weight change by the standard deviation of the baseline weight. Effect sizes (ES) were interpreted 
using the classification defined by Lipsey (1990): small effect (ES $0.00-0.32$ ), moderate effect (ES 0.33 - 0.55), or large effect (ES 0.56 and more).

\section{Results}

\section{General study characteristics}

Seven intervention studies, described in nine papers, were identified as meeting the inclusion criteria (Aragona, Cassady, \& Drabman, 1975; Golley, 2005; Golley, Magarey, Baur, Steinbeck, \& Daniels, 2007a; Golley, Perry, Magarey, \& Daniels, 2007b; HarveyBerino \& Rourke, 2003; Israel, Guile, Baker, \& Silverman, 1994; Israel, Stolmaker, \& Andrian, 1985; Robertson et al., 2008; West, 2007). General study characteristics are described in Table 3.1. The studies were published between 1975 and 2008. Three studies had been conducted in the USA (Aragona et al., 1975; Israel et al., 1994; Israel et al., 1985), one in the USA and Canada (Harvey-Berino \& Rourke, 2003) two in Australia (Golley et al., 2007a; West, 2007) and one in the UK (Robertson et al., 2008). All authors reported inclusion and/or exclusion criteria to determine who could participate in the intervention. An age limit was reported in each study as an inclusion criterion and five studies exclusively included overweight children (Golley et al., 2007a; Israel et al., 1994; Israel et al., 1985; Robertson et al., 2008; West, 2007). Six studies were based on a Randomized Controlled Trial (RCT), with participants randomly allocated to either two or three different experimental groups. One study (Robertson et al., 2008) made use of a pretest-posttest design in which all participants received the same treatment. Participants in all studies were assessed at baseline and immediately at the end of the intervention. With the exception of the study of Harvey-Berino \& Rourke (2003), study participants were also assessed after a period of no further intervention, to indicate maintenance of the intervention effects. These follow-up periods ranged from twenty weeks to three years. The drop-out rate of participants in the studies was reported per group at each measurement, ranging from 0\% (Aragona et al., 1975; Harvey-Berino \& Rourke, 2003) to 60\% (Aragona et al., 1975). The drop-out rate did not differ significantly between the experimental and control groups, except for in Aragona et al. (1975) and Harvey-Berino and Rourke (2003).

Five studies included less than 50 participants, whereas the two remaining studies included more than 100 children (mean = 52 participants). Only the study of Golley (2005) reported a sample size calculation. The unweighted mean age of the participants was 8.3 years; one study included preschool children, five studies included children aged between 5 and 11 years and one study included children older than 11 years. Most studies primarily included girls (Aragona et al., 1975; Golley et al., 2007a; Israel et al., 1985; Robertson et al., 2008; West, 2007). In six studies, all participants 
were overweight or obese, while one study (Harvey-Berino \& Rourke, 2003) also included normal-weight children. The ethnicity of the participants was reported in four studies: three included mainly Caucasians (Golley et al., 2007a; Robertson et al., 2008; West, 2007), and one study included only Native Americans (Harvey-Berino \& Rourke, 2003). 


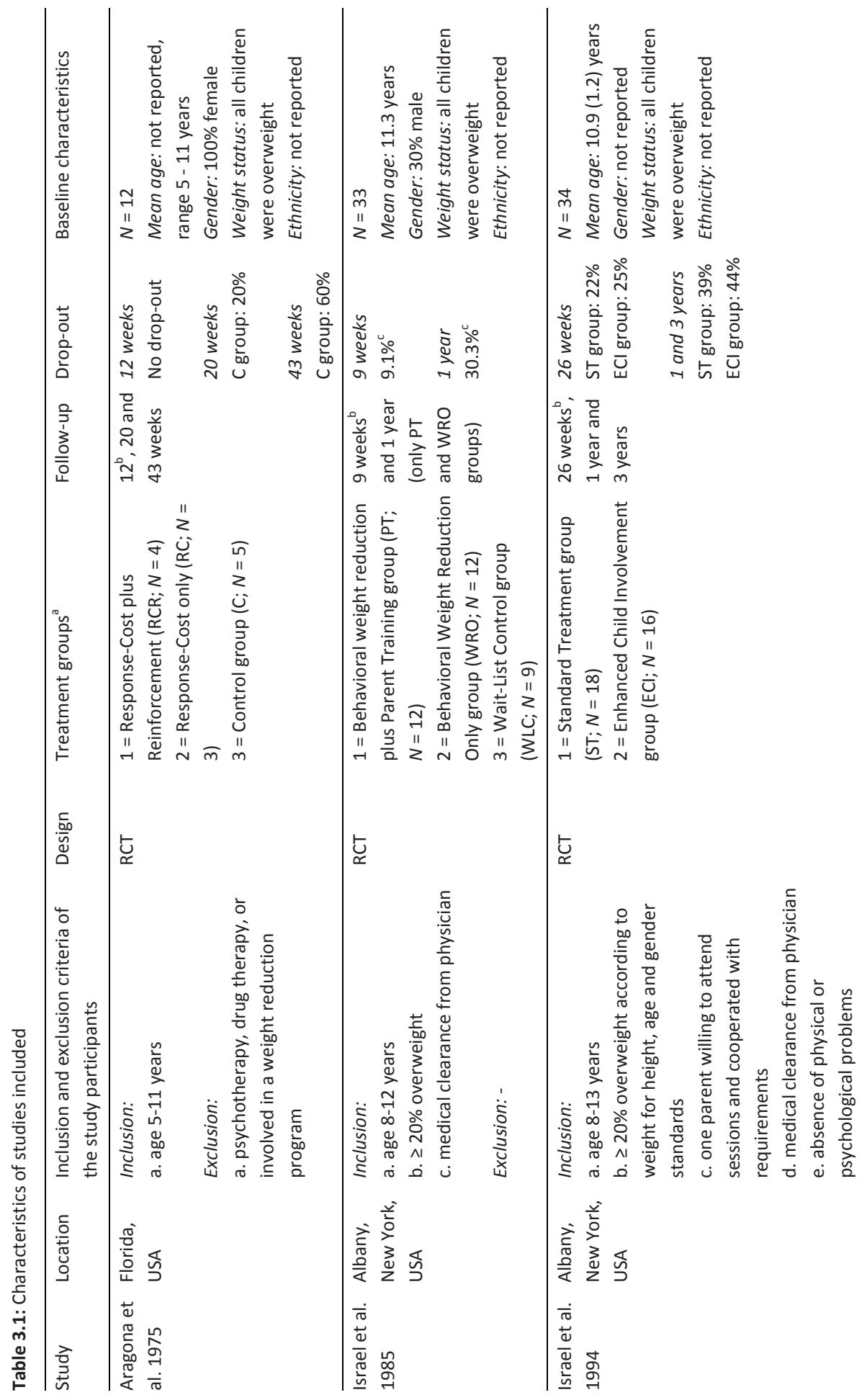




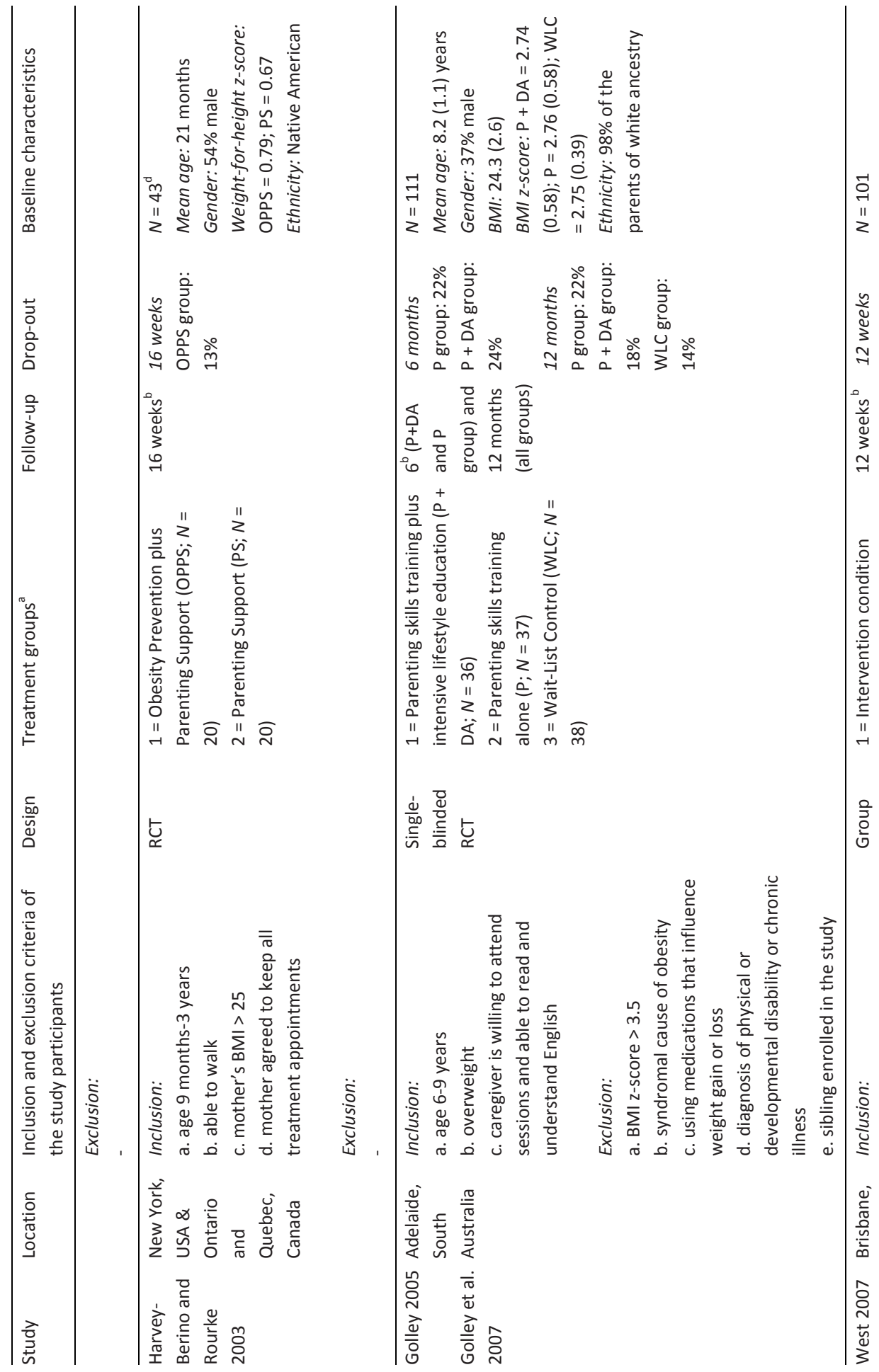




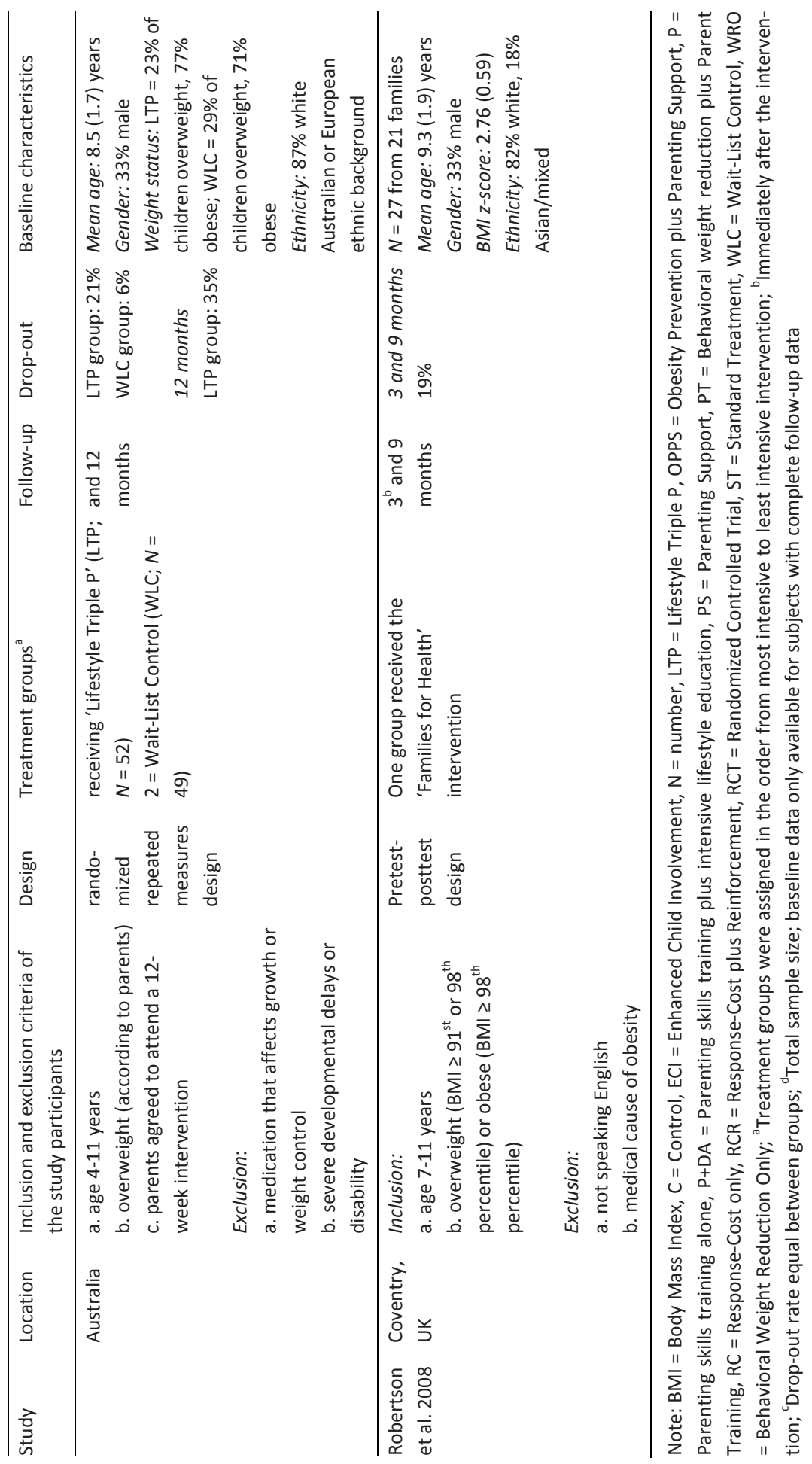




\section{Intervention description}

The interventions are described in Table 3.2. The duration of the interventions ranged from nine weeks to six months (mean = approximately 16 weeks). Four studies had separate intervention components for both parents and children: three of them offered children a multi-component program with group sessions as well as exercise components (Israel et al., 1994; Israel et al., 1985; Robertson et al., 2008); and in one study children were provided with an intensive exercise program (Maccoby \& Martin, 1983). Two studies mainly focused on the parents but did include the children (active game sessions) (Golley et al., 2007a; West, 2007), and one study did not include the children at all (Harvey-Berino \& Rourke, 2003). The interventions were delivered by different methods, but they all incorporated group sessions for parents. In four studies, the intervention was delivered by intensively trained facilitators, who were supervised during the training (Golley, 2005; Harvey-Berino \& Rourke, 2003; Robertson et al., 2008; West, 2007). In every study, the content of the intervention consisted of components relating to general parenting and specific parenting practices related to physical (in)activity and/or nutrition. The included studies used four different standardized general parenting programs, which are described below.

The first program identified was based on the book 'Living with Children' by Patterson (Patterson, 1976; Patterson \& Gullion, 1968). Based on Social Learning concepts from Skinner (1953) and Bandura (1971), the book gives parents brief, very specific instructions on how to change behaviors which almost every parent encounters. It offers programmed instructions, in which the main ideas in the book are broken down into small items on which parents have to respond by writing down an answer.

Three different studies described an intervention that provided parents with 'Living with Children' to help them acquire child management skills. Aragona et al. (1975) used the book as a guidance for the content of the group sessions with parents, while the control group did not receive the book. Israel et al. $(1994,1985)$ performed two studies in which they based the lectures in their intervention on the content of the book, while the parents were simultaneously required to read the book. In their 1985 study, only the intervention group attended sessions and read the book, whereas the control group did not receive any information about general parenting. In contrast, their 1994 study involved both the experimental and control groups attending the parent training about general child management and reading the book. The difference between the groups was that parents in the standard treatment condition were made responsible for their child's motivation and compliance with the program, whereas in the enhanced child involvement condition, the children were encouraged to manage their own weight loss efforts. 
The second general parenting program used in the intervention studies was the Parenting Positive Program (Triple P) (Golley et al., 2007a; West, 2007). Triple P is a standardized general parenting program (Sanders et al., 2003), based on social learning principles (Patterson, 1982), which aims to promote the parents' competence to manage their child's behavior. Self-management is fostered through self-evaluation and problem solving. Triple $P$ tries to enhance the knowledge, skills and confidence of parents in order to prevent behavioral, emotional, and developmental problems in their children. The basis of Triple $P$ is formed by five core parenting principles: (a) ensuring a safe and engaging environment, (b) creating a positive learning environment, (c) using assertive discipline, (d) having realistic expectations, and (e) taking care of oneself as a parent. These principles are translated into a range of positive parenting strategies: e.g., spend quality time with your child; provide engaging activities; set developmentally appropriate goals; set a good example; establish clear ground rules; give clear and calm instructions; back up instructions with logical consequences, quiet time, or timeout (Sanders et al., 2003).

Golley and colleagues (2005, 2007a, 2007b) were the first to evaluate the effects of a Triple $\mathrm{P}$ intervention on childhood obesity, in which parents took part in group sessions. Two experimental groups in their study received Triple P; one of these received additional intensive lifestyle support group sessions which focused on lifestyle knowledge and skills, and their children attended structured supervised activity sessions, while the other group only received a healthy lifestyle pamphlet on top of Triple $P$. The waiting list control group received only the healthy lifestyle pamphlet. West et al. (2007) developed a new version of this Triple P intervention, called Lifestyle Triple $P$, with a specific focus on increasing physical activity and promoting healthy eating in children. The intervention group, which was provided with Lifestyle Triple $P$, was compared with a waiting list control group who did not receive any intervention.

The third general parenting program applied in childhood overweight interventions is the Active Parenting curriculum (Mullis, 1999), which emphasizes the child's psychological and behavioral goals, logical and natural consequences, mutual respect, and encouragement techniques. Harvey-Berino \& Rourke (2003) based their intervention on this curriculum. Two groups received the parenting program: a 'parenting support' group, which involved limited discussions on eating and exercise behaviors, and an 'obesity prevention plus parenting support' group, in which the lessons focused exclusively on improvement of parenting to facilitate exercise and healthy eating behaviors.

Finally, one study used the Family Links Nurturing Program, which was originally developed and evaluated for the treatment and prevention of child abuse and neglect (Hunt, 2003). The program consists of two parts: a parenting program offered to parents and a school-based intervention. The parenting program is based on four princi- 
ples: (a) helping parents to develop appropriate expectations, (b) self-awareness and self-esteem, (c) a positive approach to discipline and (d) empathy (Barlow \& StewartBrown, 2006). Robertson et al. (2008) used elements of the parenting part of the Family Links Nurturing Program and of family lifestyle programs. 


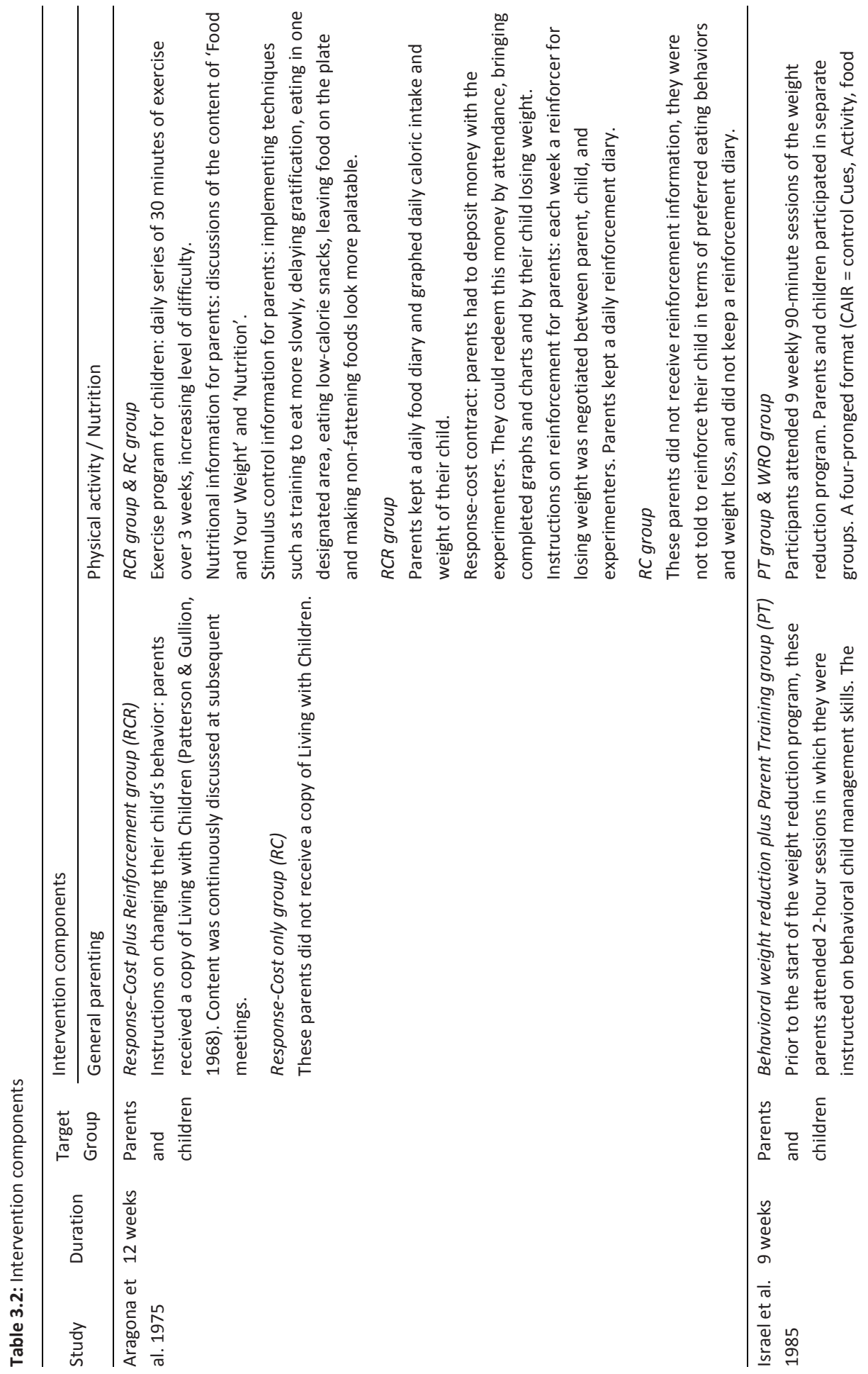




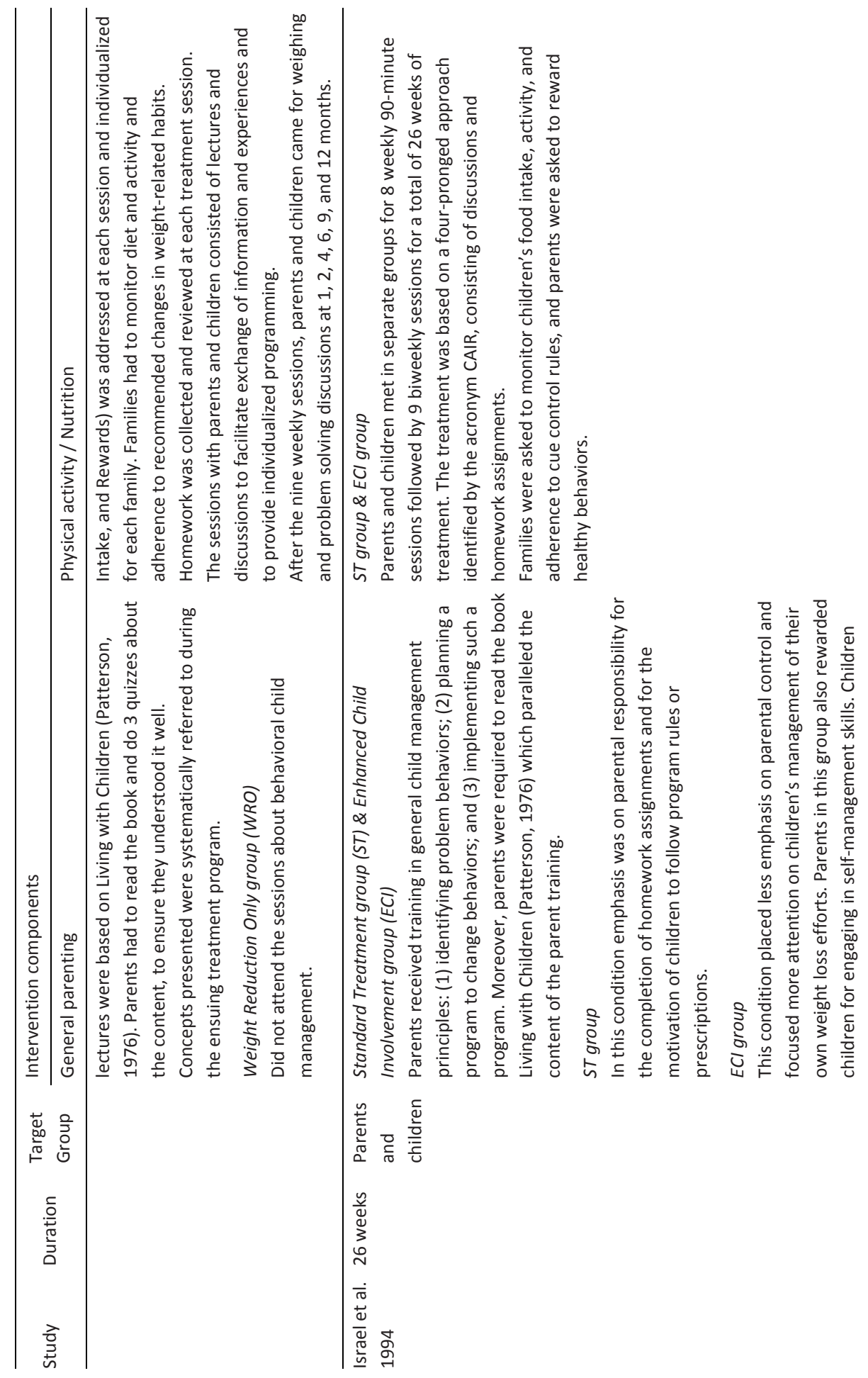




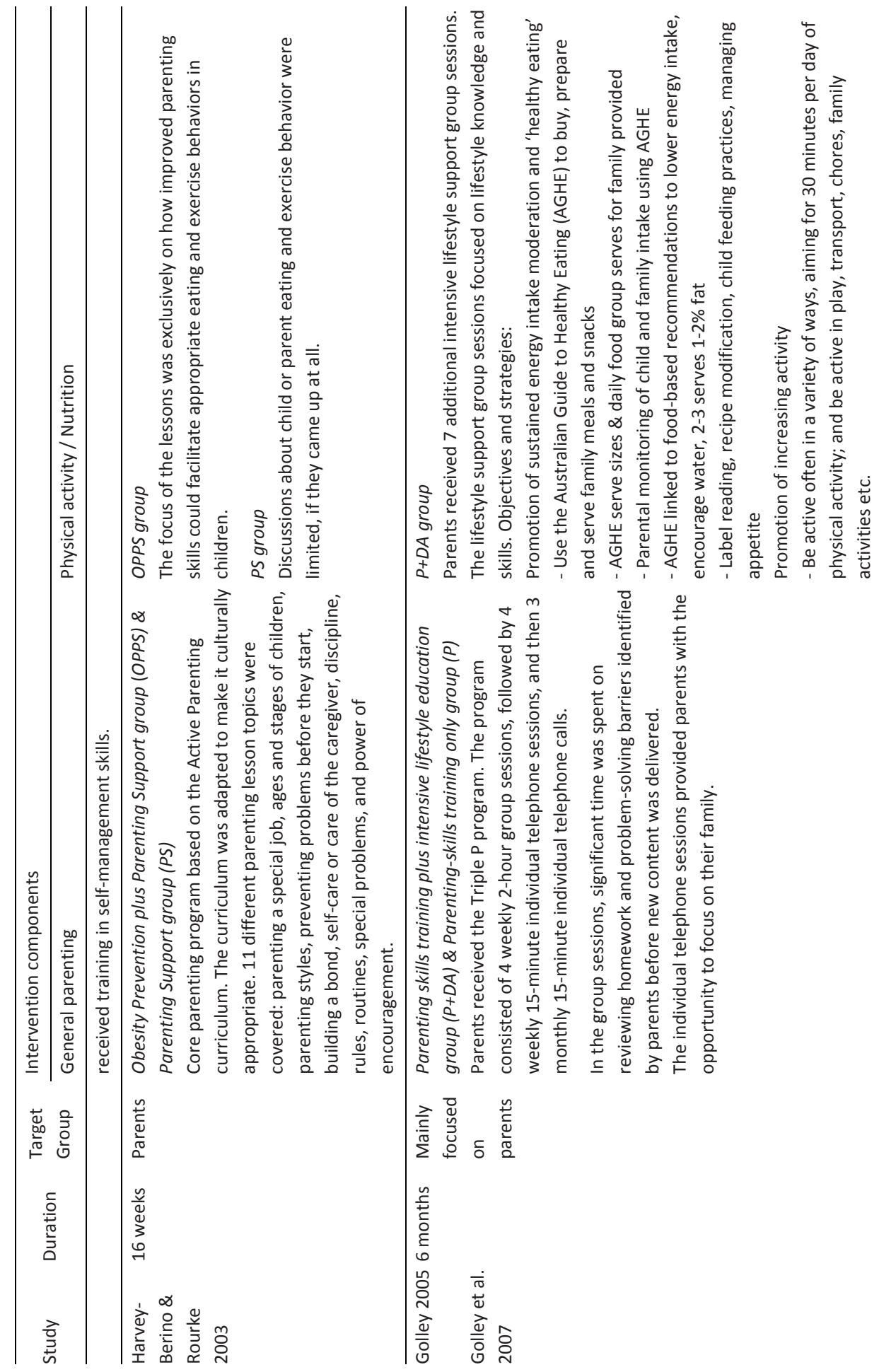




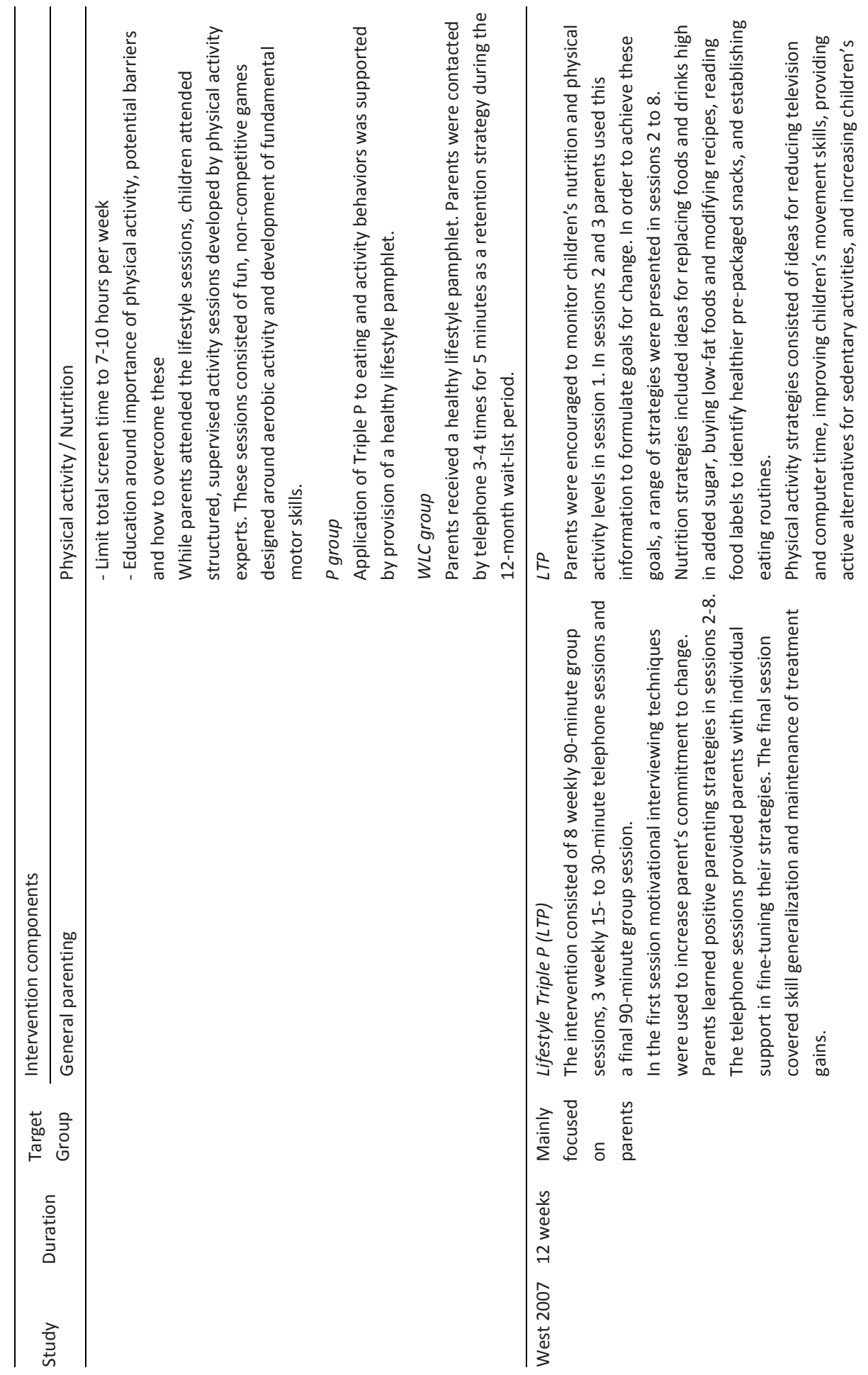




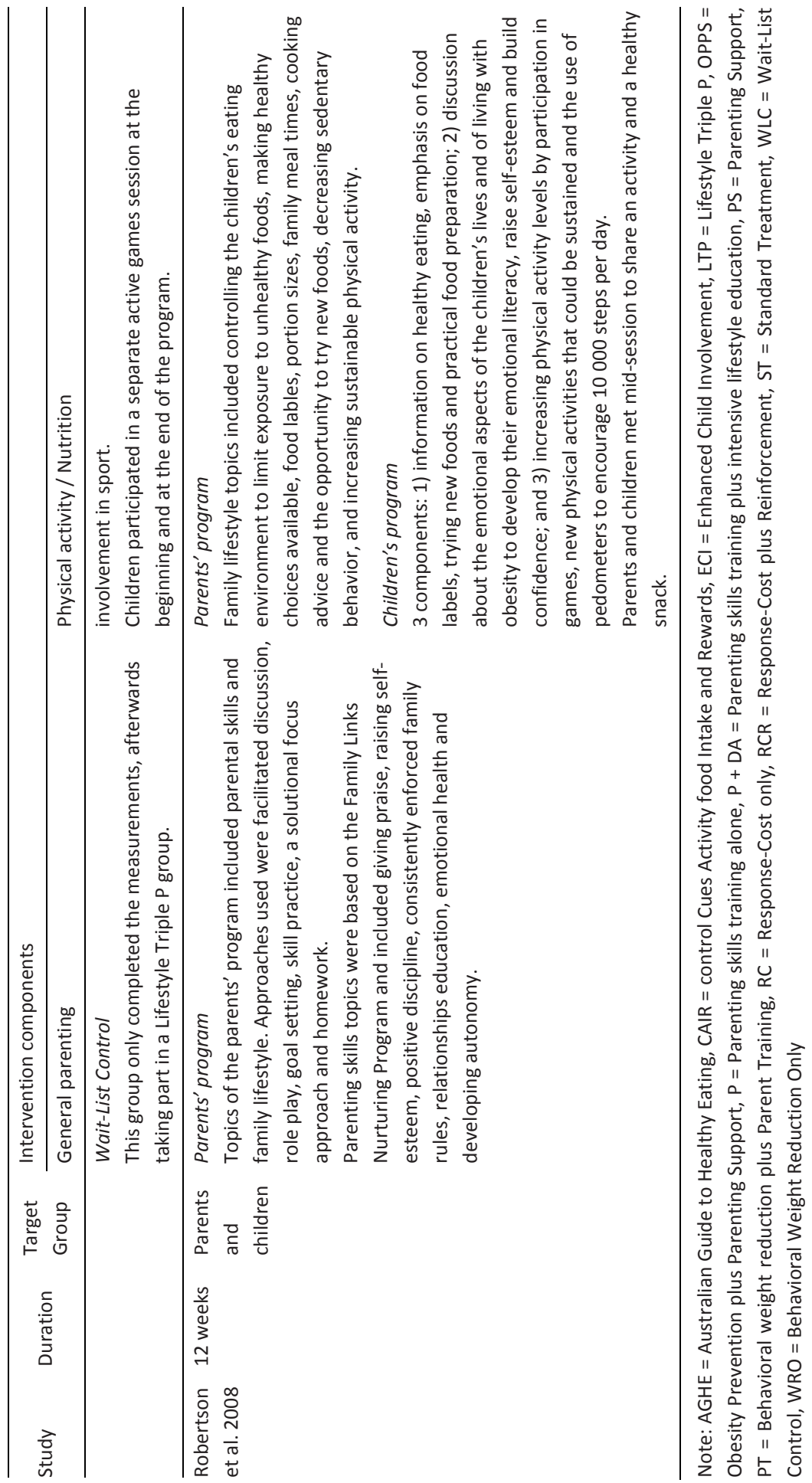




\section{Results of the interventions}

The results of the interventions are summarized in Tables 3.3 ( $a$ and b). All studies found statistically significant intervention effects on one or more anthropometric outcome measures. For five studies, the effect sizes were calculated in terms of differences in weight change between groups, based on the information provided in the published papers. The magnitude of the effect of these studies was on average small to moderate, ranging from $-0.20-0.60$. For the remaining two studies, which lacked an appropriate control group, the effect sizes were calculated in terms of change over time. The magnitude of the weight-related outcomes in these studies was on average moderate; with effect sizes ranging from $0.28-1.22$.

Four studies assessed intervention effects on energy balance-related lifestyle behaviors (physical activity, sedentary behavior and nutrition) (Golley, 2005; HarveyBerino \& Rourke, 2003; Robertson et al., 2008; West, 2007); all of these studies found significant positive effects on at least one of the behaviors measured. These positive effects were reported on energy intake, intake of extra food (high energy, fat, sugar, and/or salt food), time spent in small screen activities, time spent in active play, activity/inactivity balance, use of active transport to and from school, weekend-day sedentary activity, and number of steps counted. Eating and/or physical activity styles were measured in two studies (Israel et al., 1994, 1985). First, Israel et al. (1985) used the Eating Habit Checklist, a parental report of the degree to which the child engages in the type of eating behavior recommended by a behavioral weight reduction program, and reported a significant improvement due to the intervention. The second study (Israel et al., 1994) was one in which children's self-control regarding eating and activity-related behaviors was measured, and reported an increase in self-control in both experimental groups due to the intervention. Parenting practice measures were reported in four studies (Golley, 2005; Harvey-Berino \& Rourke, 2003; Israel et al., 1994; Robertson et al., 2008): parental control regarding weight-related behaviors was increased in both conditions in the study of Israel et al. (1994), a significant decrease of restrictive child feeding practices was measured in one intervention condition (obesity prevention plus parenting support group) by Harvey-Berino \& Rourke (2003), children's access to television after school and on weekend days was stable over time in all conditions in the study of Golley et al. (2005), and Robertson et al. (2008) reported a significant reduction of exposure to unhealthy foods in the home in their study group. Finally, the four studies that assessed general parenting (Golley, 2005; Israel et al., 1985; Robertson et al., 2008; West, 2007) all described positive effects of the intervention on this intermediary outcome. 


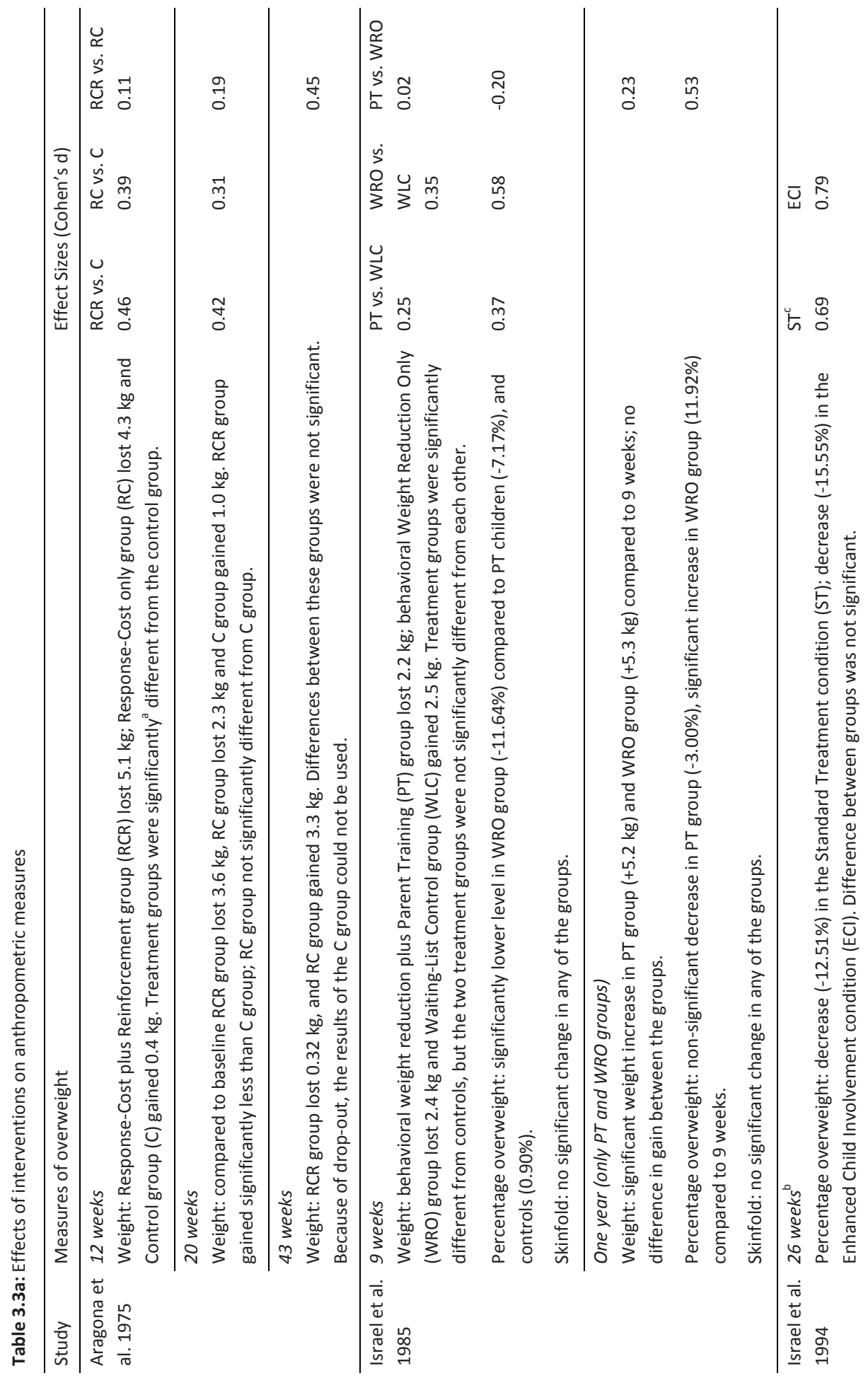




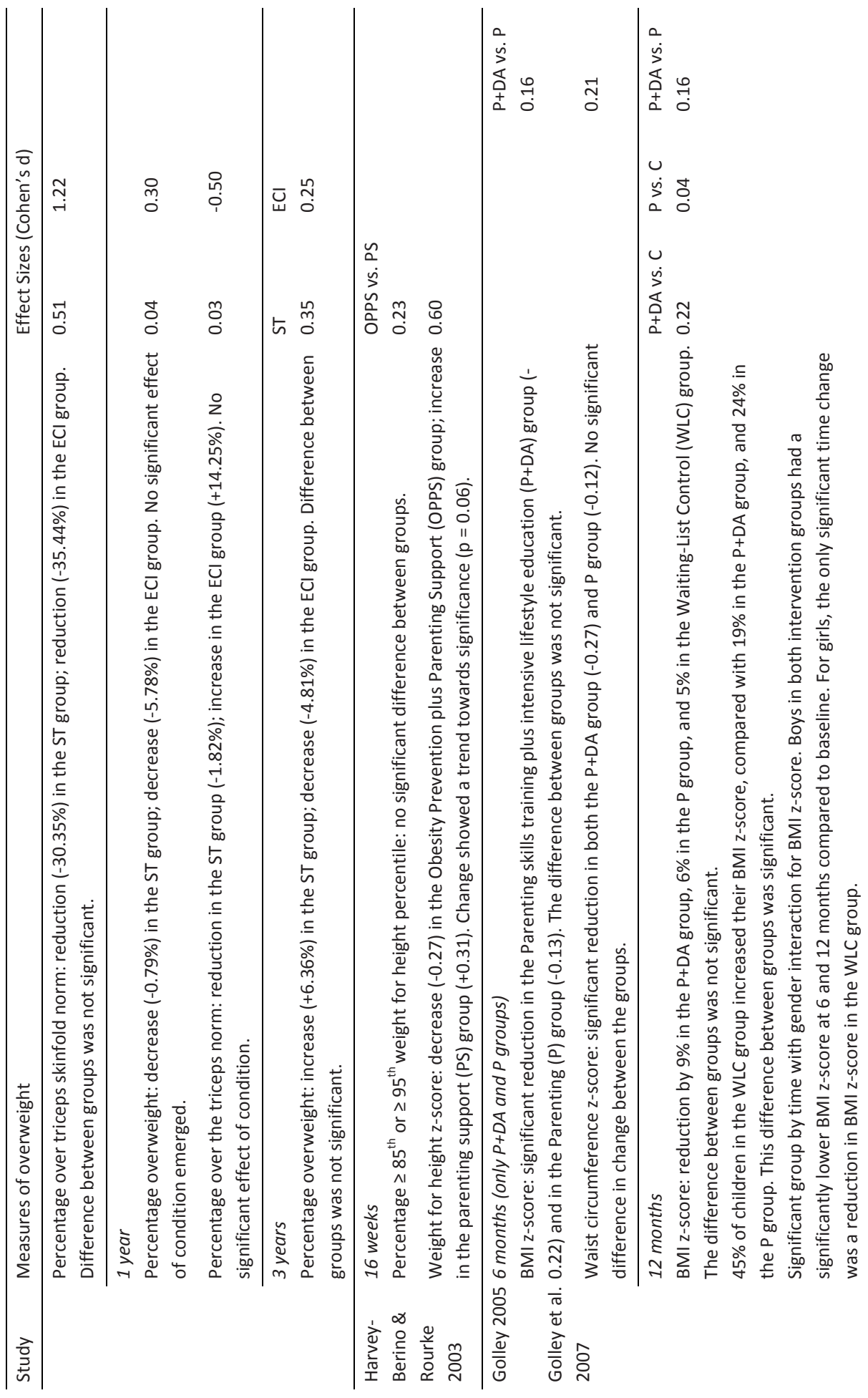


84 | CHAPTER 3

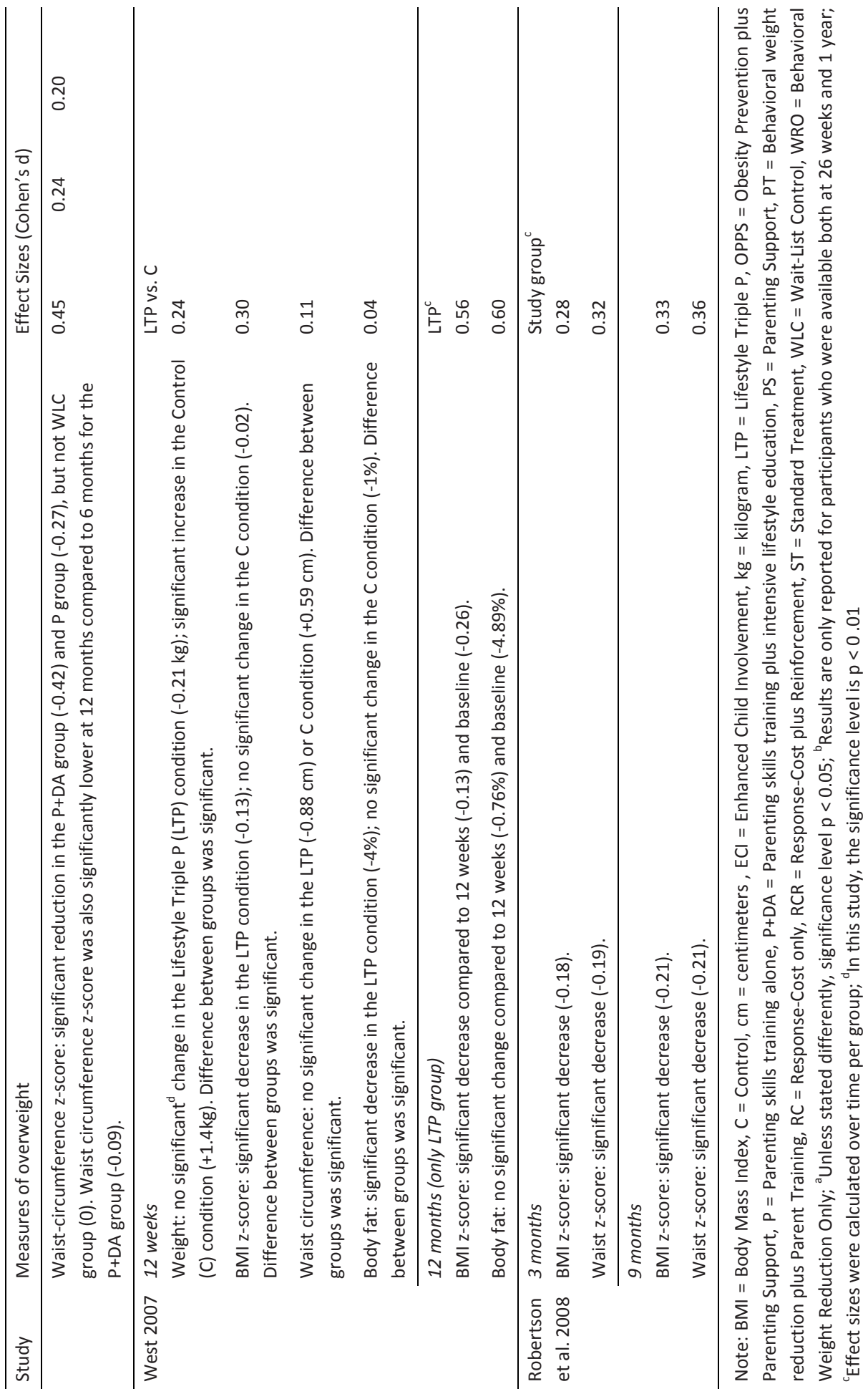




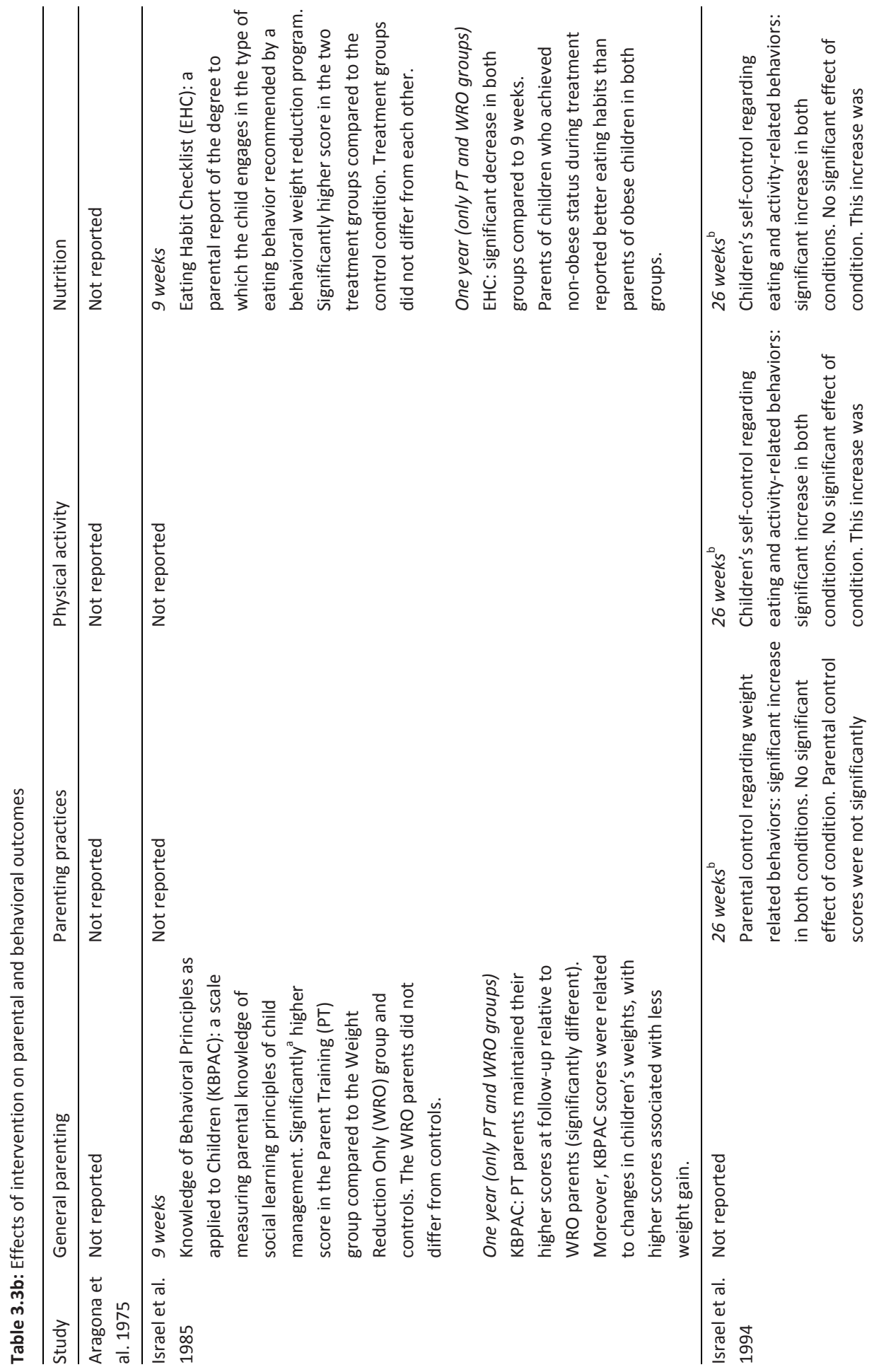


86 | CHAPTER 3

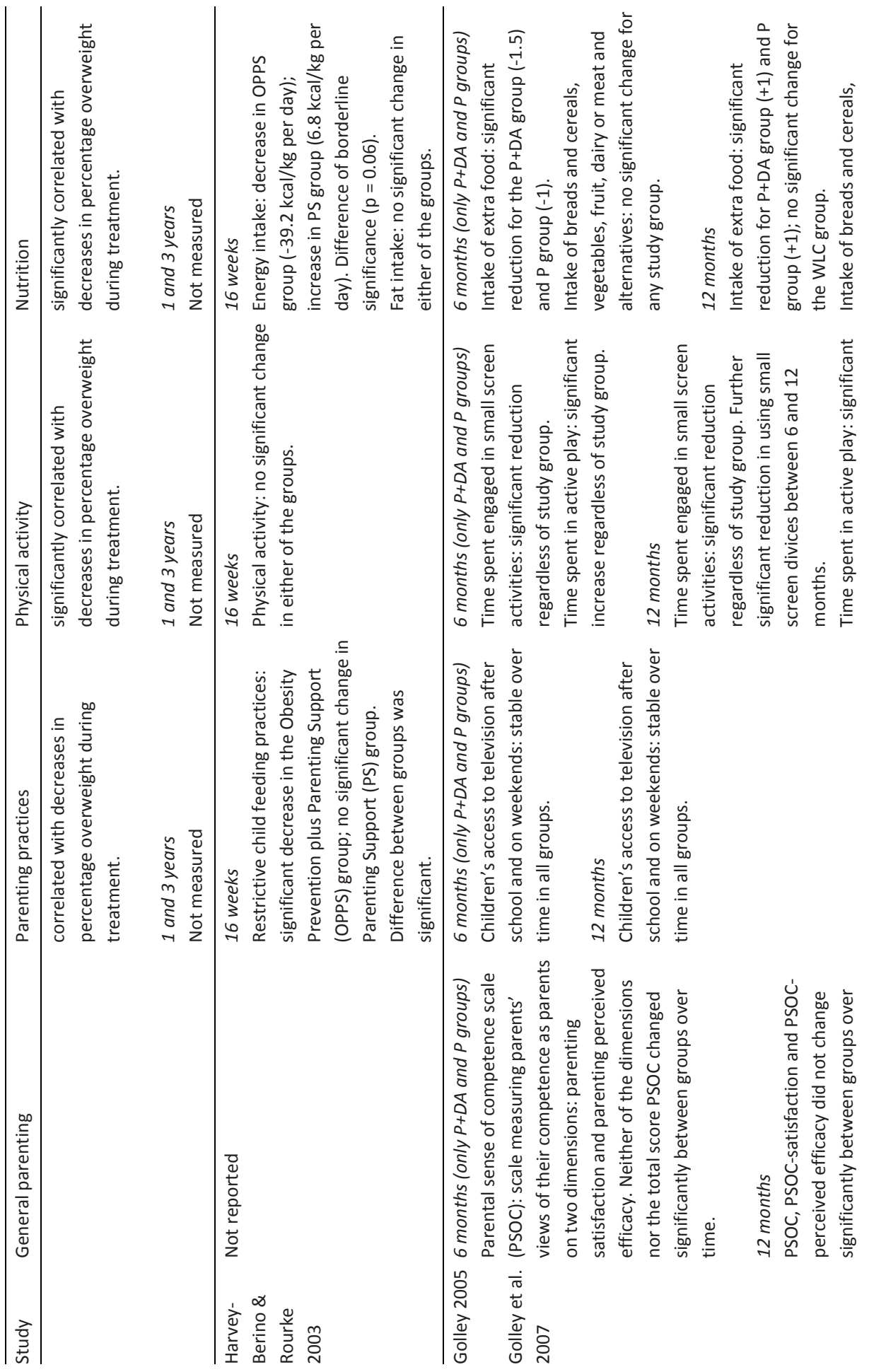




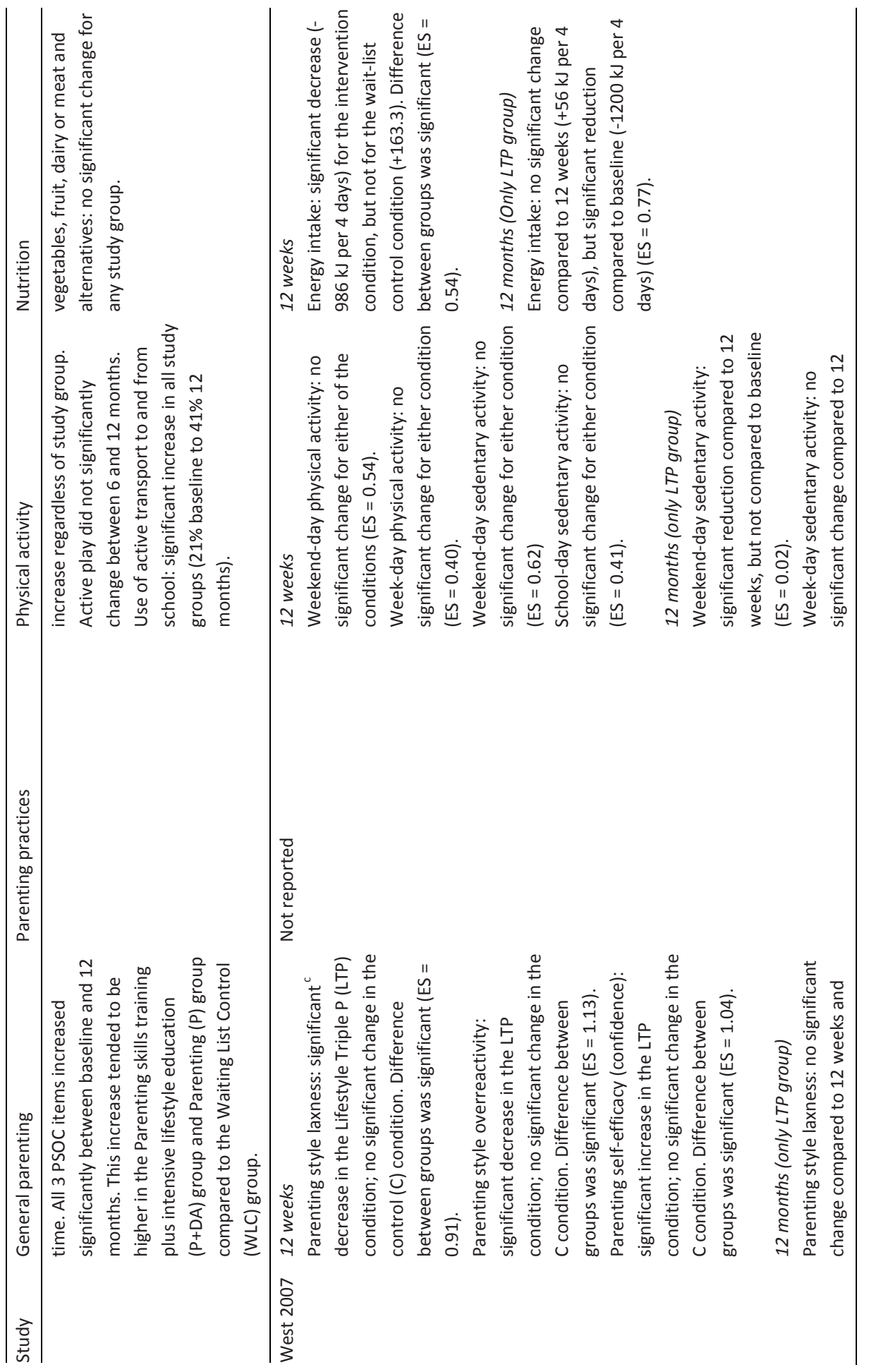




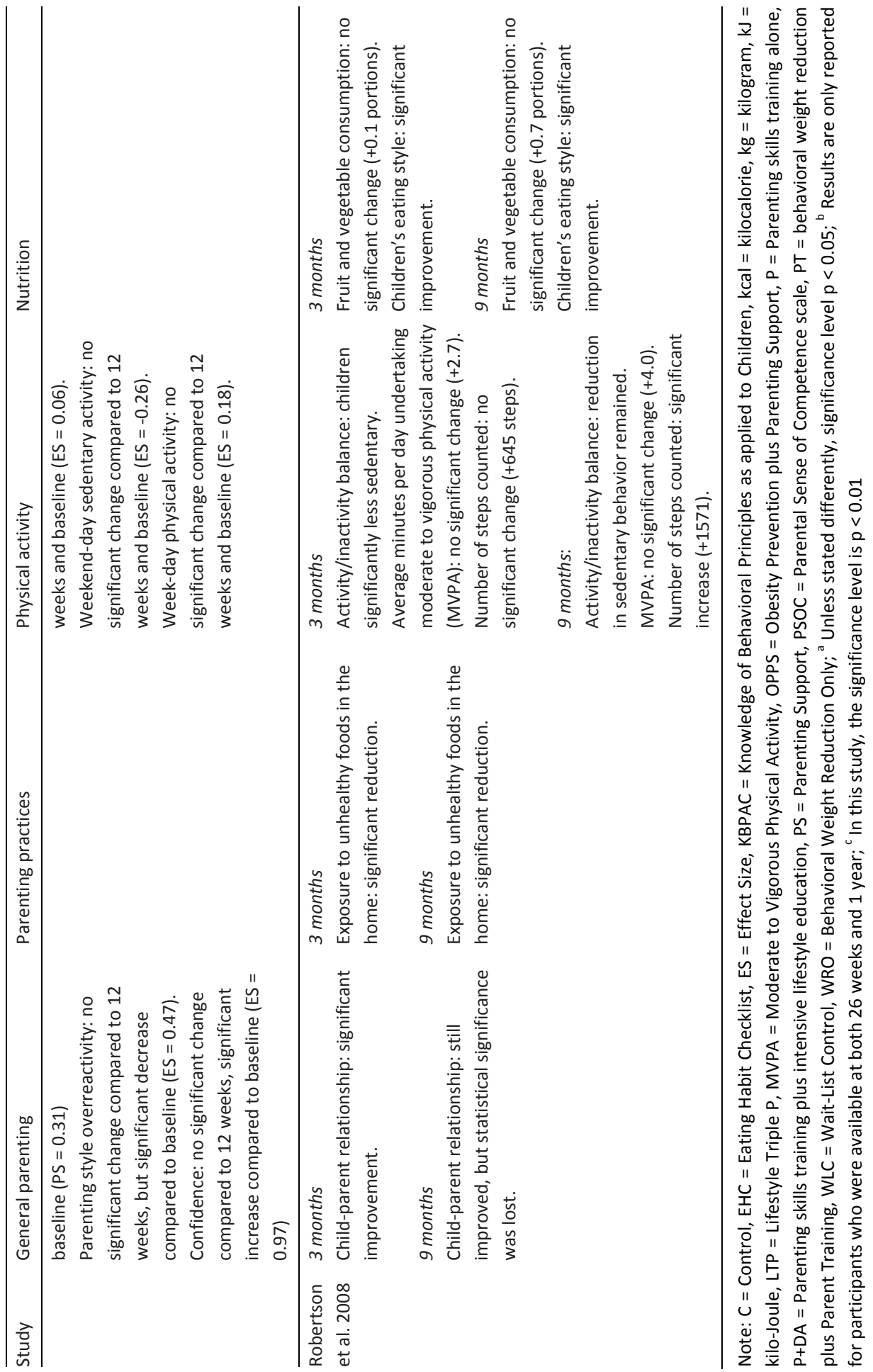




\section{Discussion}

Parenting has an important influence on the development of children's health in general (Waylen, Stallard, \& Stewart-Brown, 2008), and children's weight patterns in particular (Kitzmann et al., 2008). The current review aimed to provide an overview of interventions that address general parenting in order to prevent or treat obesity in youth. All studies showed significant small to moderate intervention effects on at least one weight-related outcome measure.

Only seven studies met the inclusion criteria for this review. However, observational studies in the field of pediatric obesity provide increasing evidence for the important role of general parenting in the development of energy balance-related behaviors and children's weight (Sleddens et al., 2011). A reason for this apparent discrepancy may be that observational research addressing general parenting in the area of pediatric obesity has a very short history: the first study investigating parenting style as a determinant of child weight status was published in 2000 (Gable \& Lutz, 2000), and the majority of studies were published between 2007 and 2010 (Epstein, McKenzie, Valoski, Klein, \& Wing, 1994a). Papers on the evaluation of systematically designed interventions in this novel field would typically (and logically) follow these studies with a time-lag of several years.

As regards the publication year of the intervention studies included in our review, it is remarkable that three studies were published more than 15 years ago $(1975,1985$ and 1994) (Aragona et al., 1975; Israel et al., 1994; Israel et al., 1985), whereas the other four studies were conducted recently, between 2003 and 2008 (Golley, 2005; Harvey-Berino \& Rourke, 2003; Robertson et al., 2008; West, 2007). The older studies all related to obesity treatment, whereas some of the more recent studies also focused on obesity prevention. This may reflect the early awareness of the importance of parenting among professionals working in the treatment of childhood obesity (tertiary prevention), whereas it took some years before a similar awareness arose in the area of primary and secondary prevention. Indeed, we identified several descriptive papers on ongoing preventive intervention studies (Daniels et al., 2009; Wolman, Skelly, Kolotourou, Lawson, \& Sacher, 2008 and A Vaughn, unpubl. data, 2010), as well as studies registered in the International Standard Randomized Controlled Trial Number Register that report incorporating general parenting in their interventions. Furthermore, the current acknowledgement of the importance of general parenting is indicated by an increasing number of childhood prevention studies assessing parenting style as an outcome measure or as a potential moderator of the intervention effect (Golan, Kaufman, \& Shahar, 2006; Shelton et al., 2007; Stark et al., 2011; Stein, Epstein, Raynor, Kilanowski, \& Paluch, 2005). In addition, even though they did not explicitly address general parenting, we have come across studies that addressed the wider 
context in which parenting practices take place (for example emotional climate), e.g., (Epstein et al., 2001a; Epstein et al., 1994a; Epstein, Paluch, \& Raynor, 2001b; Epstein, Wing, Koeske, \& Valoski, 1985; Flodmark, Ohlsson, Rydén, \& Sveger, 1993; Golan, 2006; Golan, Fainaru, \& Weizman, 1998; Golan et al., 2006; Golan, Weizman, Apter, \& Fainaru, 1998; Janicke et al., 2008; Kalarchian et al., 2009; Nowicka \& Flodmark, 2008; Nowicka, Pietrobelli, \& Flodmark, 2007).

Another reason for the observed lack of childhood obesity programs targeting general parenting may be that intervention developers are unaware or not convinced of the modifiability of general parenting. However, the intervention studies in the current review that measured parenting found large effects for this outcome measure (Golley, 2005; Israel et al., 1985; Robertson et al., 2008; West, 2007), indicating that general parenting is indeed modifiable. Research in other fields also provides evidence for the modifiability of general parenting (Spoth, Redmond, \& Shin, 1998).

We found effect sizes indicating a moderate effect on one or more outcome measures in all studies; which are considered clinically meaningful. However, in a number of these studies the changes were not statistically significant. This may also reflect issues of power and measurement precision. The results highlight that further work is needed in this area before firm conclusions can be drawn. The interventions seemed to report a relatively larger effect on general parenting. Note that general parenting is a general concept, which determines the context of behavior-specific parenting. A positive change in this variable can affect the impact on a broad range of specific parenting practices, regarding multiple child outcomes (Kitzmann \& Beech, 2006). Changes in general parenting may therefore indicate a potentially large public health effect (Spoth et al., 1998). However, it is expected that general parenting interventions are especially effective in younger children (Stice, Shaw, \& Marti, 2006). Parental influence will decrease with advancing age of their children, which makes it more difficult to intervene with these types of interventions on older adolescents. Furthermore, it is expected that parenting behaviors of parents of older children are more difficult to change because they are more likely to perform routine behaviors.

Although the content of the intervention studies included in our review varied, they did show various similarities. They all aimed to promote a parenting style that encourages instrumental competence in children by helping them balance otheroriented, rule-following tendencies with individualistic, autonomous active thinking (Baumrind, 1978). This parenting style is typically referred to as authoritative parenting (Baumrind, 1978). Second, all interventions reviewed used group sessions for parents, meaning that they are all high-intensity interventions in which parents were seen multiple times and parental interaction played a key role. Also, all intervention studies combined components of parenting styles with lifestyle education. Two studies compared an intervention primarily focusing on general parenting with an intervention 
aimed at general parenting as well as physical activity and nutrition components (Golley, 2005; Harvey-Berino \& Rourke, 2003). These studies showed that interventions combining general parenting components with lifestyle components may lead to better results than interventions focusing exclusively on general parenting. Note that several studies (Harvey-Berino \& Rourke, 2003; Israel et al., 1994; Robertson et al., 2008) did not include a control condition that excluded the general parenting program, which makes the reported results difficult to interpret in terms of the 'true' impact of adding general parenting to the intervention content.

Some limitations of the studies included in the current review should be acknowledged. Most studies used self-reported outcome measures which may evoke social desirability bias. Another limitation of the included studies was that some tools to assess energy balance-related behaviors were not validated. Furthermore, we expected that the studies which focused on general parenting also would measure general parenting as an outcome measure. This appeared not to be the case in three studies, however. We consider this apparent lack of focus on mediating mechanisms of intervention effects as undesirable. Also, the methodological quality of the included studies varied. For example, some studies had relatively small sample sizes and a relatively short follow-up period. Some studies did not apply an RCT design, while some RCTs did not include a non-intervention control group, indicating that the study quality was heterogeneous which makes it relatively difficult to compare the studies to each other. Future studies should adopt a control condition excluding the general parenting component, and include long-term follow-up. Evaluation models should include mediating concepts such as general parenting.

The current review indicates that the promotion of authoritative parenting is a valuable addition to childhood obesity. Given the lack of current intervention studies addressing general parenting, further development and testing of theory- and practicebased interventions is strongly recommended. 



\section{CHAPTER 4}

\section{Parental perception of child's weight status and subsequent BMI development:} the KOALA Birth Cohort Study

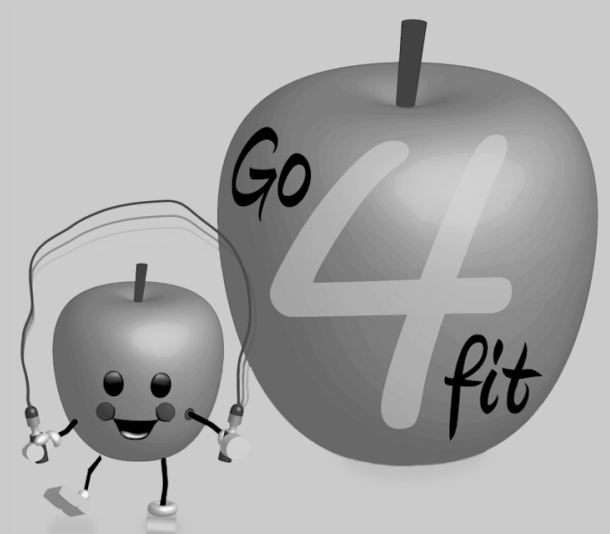

Submitted as: Gerards, S.M.P.L., Gubbels, J.S., Dagnelie, P.C., Kremers, S.P.J., Stafleu, A., De Vries, N.K., Thijs, C. Parental perception of child's weight status and subsequent BMI development: the KOALA Birth Cohort Study. 


\section{Abstract}

Introduction: Parents often fail to correctly perceive their children's weight status, but no studies have examined the association between parental weight status perception and longitudinal BMI development at various ages. We investigated whether parents are able to accurately perceive their child's weight status, at age 5 . We also investigated predictors of accurate weight status perception. Finally, we investigated the predictive value of accurate weight status perception in explaining children's longitudinal weight development up to the age of 9.

Methods: We used longitudinal data from the KOALA Birth Cohort Study. At the child's age of 5 years, parents filled out a questionnaire regarding child and parent characteristics and their perception of their child's weight status. We calculated the children's actual weight status from parental reports of weight and height at ages 2, 5, $6,7,8$, and 9 years. Regression analyses were used to identify factors predicting which parents accurately perceived their child's weight status. Finally, regression analyses were used to predict subsequent longitudinal BMI development in overweight children.

Results: Eighty-five percent of the parents of overweight children underestimated their child's weight status at age 5 . The child's BMI z-scores at age 2 and 5 were significant positive predictors of accurate weight status perception (vs. underestimation) in normal weight and overweight children. Accurate weight status perception was a predictor of higher future BMI in overweight children, corrected for actual BMI at baseline.

Conclusions: Children of parents who accurately perceived their child's weight status had a higher BMI over time, making it easier for parents to correctly perceive their child's overweight. Parental awareness of the child's overweight as such may not be sufficient for subsequent weight management by the parents, implying that parents who recognize their child's overweight may not be able to adequately manage the overweight. 


\section{Introduction}

Childhood overweight and obesity are rapidly increasing public health problems (WHO, 2011). Preventive interventions are increasingly being aimed at parents of overweight children (Golley et al., 2011), who are responsible for a substantial part of their children's nutrition and physical activity behaviors. However, parents often fail to recognize their child's overweight (Eckstein et al., 2006; Oude Luttikhuis, Stolk, \& Sauer, 2010): a systematic review on parents' perceptions of their children's weight status showed that more than half of the parents of overweight children underestimate their child's overweight, perceiving their child as normal weight (Parry, Netuveli, Parry, \& Saxena, 2008).

Several studies have examined cross-sectional factors related to parental underestimation of their child's weight status: lower child birth weight (Chaparro, Langellier, Kim, \& Whaley, 2011), a higher physical activity level of the child (Manios, Kondaki, Kourlaba, Vasilopoulou, \& Grammatikaki, 2009), having a female child (De La, 2009; Manios et al., 2009), higher age of the child (Maynard, Galuska, Blanck, \& Serdula, 2003), higher parental educational level (Baughcum, Chamberlin, Deeks, Powers, \& Whitaker, 2000; De Hoog, Stronks, Van Eijsden, Gemke, \& Vrijkotte, 2012; Manios et al., 2009), higher maternal health literacy level (Garrett-Wright, 2011), parents being a first-generation immigrant (vs. second-generation) (De Hoog et al., 2012) and higher maternal age (De Hoog et al., 2012), have been found to be associated with accurate parental perception of their child's weight status. Findings regarding the relationship between parental weight perception and children's actual BMI at that moment have been inconsistent: some studies found a positive association between children's BMI zscores and accurate weight status perception in overweight children (Manios et al., 2009; Maynard et al., 2003), whereas one study found that children's BMI z-scores were positively associated with underestimation of the children's overweight status (Yao \& Hillemeier, 2012). The relationship between the mother's weight status and perception of their children's weight is also still unclear. Some studies showed that higher maternal BMI was associated with underestimation of their children's weight status (Chaparro et al., 2011; Mamun, McDermott, O'Callaghan, Najman, \& Williams, 2008; Warschburger \& Kroller, 2009), while another study found that mothers with a lower BMI were more likely to underestimate their children's overweight (Yao \& Hillemeier, 2012). Discrepant findings may be due to different populations, sampling methods and/or definitions of overweight (Manios et al., 2009; Maynard et al., 2003).

It is also important to explore the association between parental weight status perception and subsequent BMI development of the child, as this provides an indication of whether parents are able to accurately manage their child's overweight. Kroke et al. (2006) have performed such a longitudinal investigation into the relation be- 
tween mothers' perception of the weight status of their children at different ages and the children's actual weight at age 7. They compared the BMI change in children whose mothers correctly perceived their child's weight status with that in children whose mothers did not. They showed that BMI development was only significantly different between the two groups of mothers for the 6-months-old children: maternal misperception was associated with unfavourable subsequent weight development. However, the sample sizes of the different subgroups in this study were relatively small, hampering inferences from this study.

No earlier studies have taken account of the child's prior BMI in predicting parental weight status perception. The aims of the current study were to explore whether parents are able to indicate their child's weight status (around age 5); to examine predictors of accurate parental weight status perception, including children's prior BMI at 2 years as a predictor; and to investigate children's longitudinal subsequent BMI development up to age 9 years in relation to parental perceptions or misperceptions of their child's weight status.

\section{Methods}

\section{Respondents and procedure}

The KOALA Birth Cohort Study is a prospective cohort study in the Netherlands which started in 2000. KOALA is a Dutch acronym for Child, Parent, and health: Lifestyle and Genetic constitution. Pregnant women were recruited from an existing cohort from a study of pregnancy-related pelvic girdle pain. Additional participants were recruited among 'alternative lifestyle' circles (e.g., organic food shops, anthroposophist midwives) (Kummeling et al., 2005). This latter group of women could have an alternative lifestyle as regards aspects like child rearing, dietary habits, vaccination schemes or antibiotics use.

The Medical Ethics Committee of the University Hospital and Maastricht University approved the study. All participants signed an informed consent form. A total of 2834 mothers participated and completed questionnaires during pregnancy as well as at regular intervals after birth.

\section{Questionnaire}

In the current study, we analyzed child and parental characteristics by means of a questionnaire which parents completed when the children were approximately 5 years old. The total number of questionnaires returned was 2066 (73\%). In addition, we analyzed parental reports of the weight and height of their children at the ages of around $2(\mathrm{~N}=1735 ; 84 \%$ of the respondents at age 5$), 5(\mathrm{~N}=1915 ; 93 \%), 6(\mathrm{~N}=1475$; 
$71 \%), 7(\mathrm{~N}=1362 ; 66 \%), 8(\mathrm{~N}=1288 ; 62 \%)$ and $9(\mathrm{~N}=1394 ; 67 \%)$. The weight and height of a substantial proportion of the children $(\mathrm{N}=372)$ at the age of 5 were also measured by trained research assistants (Eijkemans et al., 2008).

\section{Weight status perception}

Parental perception of their child's weight status was assessed around age 5 by the following questions: 'How would you describe your child's weight currently'. Answer categories were: clearly underweight, underweight, normal weight, overweight and clearly overweight. In view of the very small proportions of children classified in the two extreme categories, we recoded the answers into three categories: underweight (including both clearly underweight and underweight), normal weight and overweight (including both overweight and clearly overweight).

\section{Parental characteristics}

At the child's age of 5, both parents were asked to report their own weight and height, which were used to calculate their body mass index (BMl; weight $(\mathrm{kg}) /(\text { height }(\mathrm{m}))^{2}$ ). A BMI below $18.5 \mathrm{~kg} / \mathrm{m}^{2}$ was regarded as underweight, and a BMI above $25 \mathrm{~kg} / \mathrm{m}^{2}$ as overweight. Additionally, parents were asked to indicate the number of hours they worked per week, their country of birth and their highest completed level of education. Country of birth was recoded into Netherlands vs. other. Education was categorized into three levels: low, medium and high, based on international classification systems (Eurostat., 2007) (low: primary school, lower vocational education and lower general secondary education; medium: intermediate vocational education, higher general secondary education and university preparatory education; high: higher vocational education and university). From previous questionnaires, we derived the mother's age at the birth of the child and the recruitment channel (alternate vs. regular).

\section{Child characteristics}

The child's date of birth and gender were derived from previous questionnaires. Furthermore, parents were asked to report their child's weight and height in questionnaires they completed around the child's ages of 2, 5, 6, 7, 8 and 9, which we used to calculate the child's BMI. BMI was recoded into BMI z-scores standardized for age and gender, compared to the national reference population (i.e., the Fourth Dutch National Growth Study) (Fredriks, Van Buuren, Wit, \& Verloove Vanhorick, 2000). Weight status was recoded into three categories, based on BMI z-scores: underweight $\left(<5^{\text {th }}\right.$ percentile, $\mathrm{BMI} z=1.04)$, normal weight $\left(5^{\text {th }}-84^{\text {th }}\right.$ percentile, BMI $\left.1.05-1.64\right)$ and overweight $\left(\geq 85^{\text {th }}\right.$ percentile, $\left.\mathrm{BMI} z=1.65\right)$ (Barlow, 2007). 


\section{Data analyses}

All statistical analyses were conducted using SPSS 19.0. P-values $<0.05$ were considered statistically significant. We performed descriptive analyses (means and frequencies) to summarize child and parent background characteristics. Crosstabs were used to compare the distribution of parents who accurately perceived their child's weight and those who did not at age 5 years, for children of different weight categories. Children who were currently underweight were excluded from further analyses, because they were not our target population.

\section{Predictors of accurate weight status perception}

Multiple backward binary logistic regressions were conducted to predict which parents correctly estimated their child's weight status cross-sectionally at age 5 ( 1 = accurate perception of child's weight status, $0=$ underestimation of child's weight status), using child characteristics (BMI z-score at age 2, BMI z-score at age 5, gender) as well as parental characteristics (BMI of both parents, age of mother at birth of child, educational level of both parents, country of birth of both parents, employment status of both parents (hours per week) and recruitment channel) as predictors. This was done separately for children with normal weight and overweight at the age of 5 . The predictors were backward-deleted from the model based on their significance level, starting with the predictor with the highest P-value, until only statistically significant predictors were left. We performed separate analyses including an interaction term between measurement method (weight and height by parental report vs. measured by a trained research assistant) and BMI z-score in the models, to check whether the results were influenced by the measurement method used for weight and height. In none of the models was a significant interaction effect found between measurement method (selfreport vs. measured) and BMI z-scores (P-values all > 0.05).

\section{Longitudinal BMI development}

Multivariate backward linear regression analyses were performed for the children who were overweight at age 5 , to examine the relationship between accurate parental classification of their child's weight status at age 5 and subsequent changes in the child's BMI z-scores over time (from age 5 to $6,7,8$ and 9 years). The following covariates were included in the basic model: gender of the child, BMI of both parents, educational level of both parents, country of birth of both parents, employment status of both parents (hours per week) and recruitment channel. Again, the predictors were backward-deleted from the model until only statistically significant predictors were left. We included an interaction term between measurement method (self-report vs. measured) and accurate weight status perception in the analysis to investigate whether we should conduct separate analyses for children whose weight and height were 
measured and children whose weight and height were self-reported by their parents. In none of the models was a significant interaction effect found between measurement method and accurate weight status perception (P-values all $>0.05$ ).

Finally, we entered an interaction term between weight status perception and BMI z-scores at age 5 into these regression models, to explore whether BMI development was significantly different for children whose parents accurately estimated their child's weight status as compared to children whose parents underestimated their weight status.

\section{Results}

\section{Study population}

Table 4.1 lists the general characteristics of the children and parents for whom both questionnaire data and BMI z-scores were available at age 5 . At that age, $83 \%$ of the children had a normal weight and $9 \%$ were overweight (or obese). Almost all parents had been born in Netherlands, and $54 \%$ had a high educational level. Thirty-two percent of the mothers and $46 \%$ of the fathers were overweight or obese. Attrition at 6,7 , 8 and 9 years was non-selective with regard to BMI z-score and parental perception of their child's weight status at 5 years.

\section{Weight status perception}

Table 4.2 presents the distribution of parental perceptions of their child's weight status and the child's actual weight status at the age 5 . A large majority (93\%) of the parents perceived their child's weight status at 5 years to be normal. Even parents of overweight and underweight children mainly perceived their child as having a normal weight. A substantial proportion (78\%) of the parents of underweight children overestimated their child's weight status, whereas $85 \%$ of the parents of overweight children underestimated the weight status of their child.

\section{Predictors of accurate weight status perception}

Table 4.3 presents predictors of accurate parental perception of their child's weight status at age 5 . BMI z-scores at ages 2 and 5 in both the overweight and normal weight sample were significant predictors of accurate weight status perception. Compared to children with lower BMI z-scores at 5 years, parents of children with higher BMI zscores were more likely to correctly perceive their child as normal weight or overweight. In addition, parents of children whose BMI z-score was higher at 2 years were more likely to accurately perceive their child's weight status at 5 years than parents 
whose child had a lower BMI z-score at the age of 2. In normal weight children, a high paternal educational level was a significant predictor of accurate perception of the child's weight status. None of the other demographic variables showed a significant association with the accuracy of the weight status perception.

Table 4.1: Demographics and weight-related characteristics of children and parents of the KOALA cohort around age $5(\mathrm{~N}=1915)$

\begin{tabular}{|c|c|c|c|}
\hline & & Mean $\pm S D$ & Prevalence \\
\hline \multirow[t]{7}{*}{ Child } & Gender & & Male: $51 \%$ \\
\hline & & & Female: $49 \%$ \\
\hline & Age & $5.01 \pm 0.53$ & \\
\hline & BMI z-score & $-0.27 \pm 0.99$ & \\
\hline & Weight status & & Underweight: $9 \%$ \\
\hline & & & Normal weight: $83 \%$ \\
\hline & & & Overweight + Obesity: 9\% \\
\hline \multirow[t]{12}{*}{ Mother } & Age at birth of child & $32.21 \pm 3.78$ & \\
\hline & Country of birth & & Netherlands: $97 \%$ \\
\hline & & & Other: $3 \%$ \\
\hline & Educational level & & High: 54\% \\
\hline & & & Medium: $38 \%$ \\
\hline & & & Low: $8 \%$ \\
\hline & Employment (hours per week) & $17.87 \pm 10.93$ & \\
\hline & Alternative lifestyle & & $18 \%$ \\
\hline & BMI & $23.94 \pm 3.79$ & \\
\hline & Weight status & & Underweight: $2 \%$ \\
\hline & & & Normal weight: $67 \%$ \\
\hline & & & Overweight + Obesity: $32 \%$ \\
\hline \multirow[t]{10}{*}{ Father } & Country of birth & & Netherlands: $96 \%$ \\
\hline & & & Other: $4 \%$ \\
\hline & Educational level & & High: $53 \%$ \\
\hline & & & Medium: $34 \%$ \\
\hline & & & Low: $13 \%$ \\
\hline & Employment (hours per week) & $37.89 \pm 9.94$ & \\
\hline & BMI & $25.07 \pm 3.09$ & \\
\hline & Weight status & & Underweight: 1\% \\
\hline & & & Normal weight: $53 \%$ \\
\hline & & & Overweight + Obesity: $46 \%$ \\
\hline
\end{tabular}


Table 4.2: Parental cross-sectional perception of child's weight status at age 5 years by the child's actual weight status at age 5 years

\begin{tabular}{lllll}
\hline \multirow{2}{*}{$\begin{array}{l}\text { Parental perception of child's } \\
\text { weight status }\end{array}$} & \multicolumn{2}{l}{ Actual weight status of child } & \multirow{2}{*}{ Total } \\
\cline { 2 - 3 } & Underweight & Normal weight & Overweight & \\
\hline Underweight & $\mathbf{3 6 ( 2 2 \% )}$ & $67(4 \%)$ & $2(1 \%)$ & $105(6 \%)$ \\
Normal weight & $125(78 \%)$ & $1504(96 \%)$ & $141(83 \%)$ & $1770(93 \%)$ \\
Overweight & 0 & $3(0 \%)$ & $\mathbf{2 6 ( 1 5 \% )}$ & $29(2 \%)$ \\
Total & $161(100 \%)$ & $1574(100 \%)$ & $169(100 \%)$ & $1904^{a}$ \\
\hline
\end{tabular}

Note: Accurate weight perception in total sample $=82 \%$ (see bold numbers in the table); ${ }^{a} \mathrm{~N}$ deviates from total sample because of missing values

Table 4.3: Odds ratios for predictors of accurate parental perception of child's weight status at 5 years

\begin{tabular}{|c|c|c|}
\hline & \multicolumn{2}{|c|}{ Accurate parental weight status perception ${ }^{a}$} \\
\hline & \multicolumn{2}{|l|}{ Odds ratio $(95 \% \mathrm{Cl})$} \\
\hline & $\begin{array}{l}\text { Normal weight children } \\
N=1369\end{array}$ & $\begin{array}{l}\text { Overweight children } \\
N=146\end{array}$ \\
\hline BMI z-score at 2 years & $2.39 * * *(1.72 ; 3.32)$ & $2.88^{* * *}(1.58 ; 5.26)$ \\
\hline BMI z-score at 5 years & $2.92 * * *(1.72 ; 4.95)$ & $13.86 * * *(3.77 ; 50.93)$ \\
\hline \multicolumn{3}{|c|}{ Educational level of father } \\
\hline high vs medium & $1.83 *(1.00 ; 3.35)$ & $-b$ \\
\hline low vs medium & $1.53(0.62 ; 3.74)$ & $-b$ \\
\hline
\end{tabular}

Note: ${ }^{*} \mathrm{p} \leq 0.05 ;{ }^{* *} \mathrm{p} \leq 0.01 ; * * \mathrm{p} \leq 0.001$; Results of multivariate backwards binary logistic regression analyses. Non-significant $(P>0.05)$ predictors were backward-deleted. Variables that were excluded from all models due to non-significance were child's gender, BMI of both parents, recruitment channel, educational level of the mother, country of birth of both parents, employment status of both parents and age of mother at birth of child

${ }^{\mathrm{a}} 1$ = accurate perception of child's weight status, 0 = underestimation of child's weight status

${ }^{b}$ Variable not included in the final backwards regression model (n.s.).

\section{Longitudinal BMI development of 5-year-old overweight children}

We predicted the changes in BMI z-scores from age 5 to ages $6,7,8$ and 9 in overweight children (see Table 4.4). Significant predictors of changes in BMI z-scores in all four models were accurate weight status perception at age 5 and BMI z-score at age 5 . Accurate weight status perception at 5 years was associated with a greater increase in BMI z-score from 5 years to 6, 7, 8 and 9 years, compared to underestimation of the child's weight status. In addition, BMI z-score at 5 years was negatively associated with changes in BMI z-score from age 5 to ages $6,7,8$ and 9 years. 
Table 4.4: Predictors of change in child's BMI z-scores from age 5 up to age 9 for children who were actually overweight at age 5

\begin{tabular}{lllll}
\hline & \multicolumn{2}{l}{ Unstandardized regression coefficients $\mathrm{B}(95 \% \mathrm{Cl})$} \\
& 6 years & 7 years & 8 years & 9 years \\
& $N=129$ & $N=110$ & $N=103$ & $N=102$ \\
\hline Accurate perception of child's weight status & $0.70 * * *$ & $0.96 * * *$ & $0.89 * * *$ & $0.99 * * *$ \\
(O = underestimation, 1 = accurate) & $(0.31 ; 1.08)$ & $(0.56 ; 1.36)$ & $(0.41 ; 1.37)$ & $(0.48 ; 1.49)$ \\
Child BMI z-score at 5 years & $-0.57 * *$ & $-0.97 * * *$ & $-0.81 * * *$ & $-0.84 * * *$ \\
& $(-0.94 ;-0.21)$ & $(-1.34 ;-0.60)$ & $(-1.27 ;-0.37)$ & $(-1.33 ;-0.35)$ \\
\hline Total variance explained by the model $\left(\mathrm{R}^{2}\right)$ & 0.18 & 0.29 & 0.22 & 0.24 \\
\hline
\end{tabular}

Note: ${ }^{*} \mathrm{P} \leq 0.05 ;{ }^{* *} \mathrm{P} \leq 0.01 ;{ }^{* * *} \mathrm{P} \leq 0.001$; Results of multivariate backwards linear regression analyses. Nonsignificant $(P>0.05)$ predictors were backward-deleted. Variables that were excluded from all models due to non-significance were gender of the child, recruitment channel, country of birth of both parents, employment status of both parents, mother's BMI and age of mother at the birth of the child. Analyses were controlled for significant background characteristics: educational level of father, educational level of mother and BMI of father

We found a significant interaction between BMI z-score at age 5 and the perception of a child's weight status (accurate vs. inaccurate) in terms of predicting the child's change in weight from age 5 to ages 6 and $7(\beta=1.116 ; P=0.030$ and $\beta=1.608 ; P=$ 0.004 , respectively). Figure 4.1 shows the longitudinal change in BMI z-scores (based on means) of the overweight children whose parents underestimated their child's weight status and of the children whose parents correctly perceived their child's weight status at age 5 (see Table 4.5 for means and standard deviations). Children whose parents accurately perceived their weight status had a higher BMI z-score at 5 years and were more likely to maintain this higher weight, whereas children whose parents underestimated their weight had only one peak in their weight, at age 5 years.

Table 4.5: means and standard deviations for Figure 4.1

\begin{tabular}{|c|c|c|c|c|}
\hline & \multicolumn{2}{|c|}{$\begin{array}{l}\text { Underestimation of } \\
\text { weight status at } 5 \text { years }\end{array}$} & \multicolumn{2}{|c|}{$\begin{array}{l}\text { Accurate weight status } \\
\text { perception }\end{array}$} \\
\hline & Mean & SD & Mean & SD \\
\hline BMI z-score, 2 years & 0.79 & 1.01 & 1.88 & 0.87 \\
\hline BMI z-score, 5 years & 1.40 & 0.33 & 1.90 & 0.53 \\
\hline BMI z-score, 6 years & 0.76 & 0.77 & 1.66 & 0.74 \\
\hline BMI z-score, 7 years & 0.56 & 0.75 & 1.56 & 0.70 \\
\hline BMI z-score, 8 years & 0.61 & 0.86 & 1.54 & 0.66 \\
\hline BMI z-score, 9 years & 0.59 & 0.88 & 1.58 & 0.70 \\
\hline
\end{tabular}




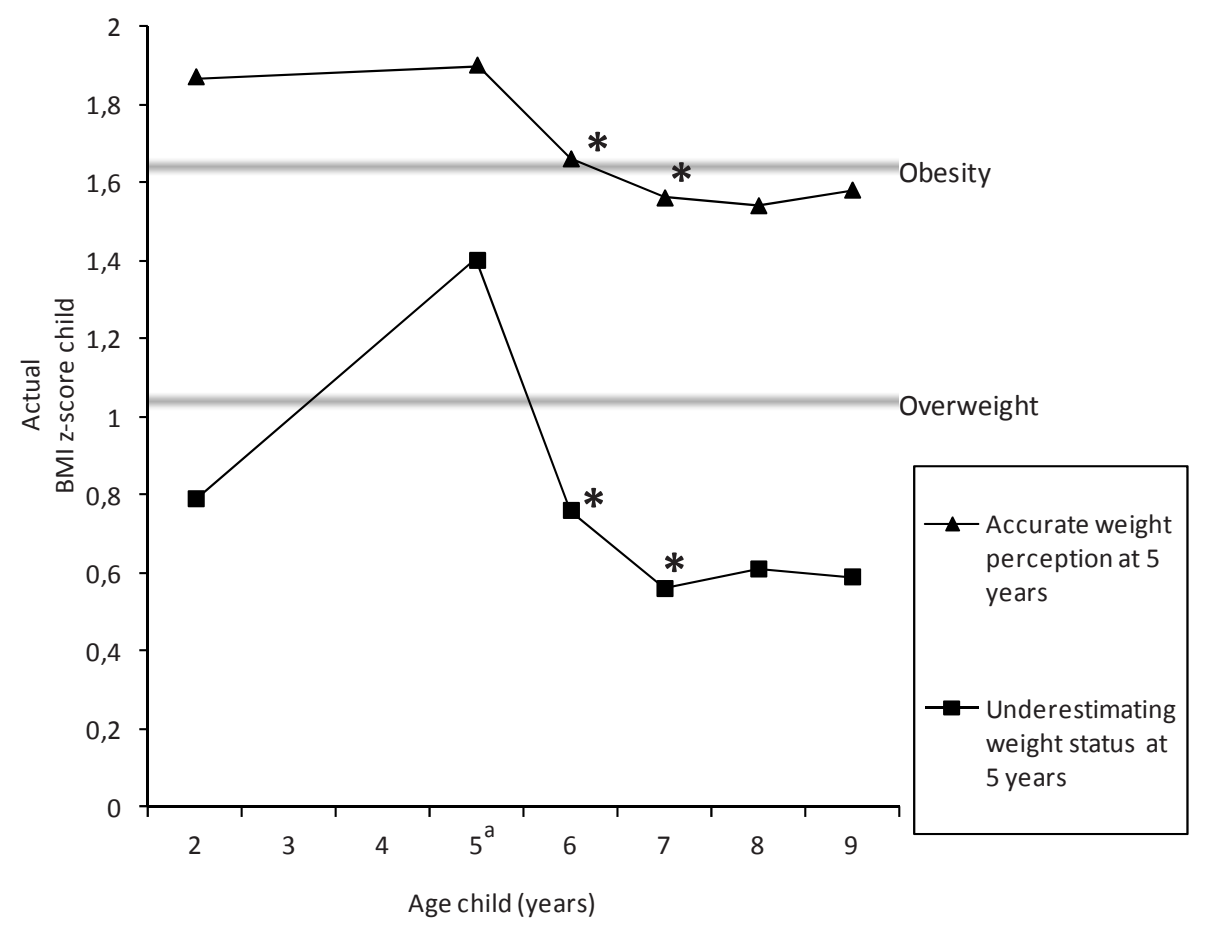

Figure 4.1: BMI development of overweight children whose parents accurately perceived or underestimated their child's weight status at the age of 5.

Note: ${ }^{a}$ Weight status perception was assessed at the child's age of 5; cut-off points for overweight and obesity were $\geq 85$ th percentile (BMI z-score $=1.04$ ) and $\geq 95$ th percentile (BMI z-score $=1.65$ ), respectively. *Significantly different patterns between the two groups in BMI change from age 5 to ages 6 and 7

\section{Discussion}

This study has yielded information about the longitudinal development of children's $\mathrm{BMI}$ in relation to parental perception of their child's weight status. We found that accurate weight status perception at age 5 was significantly associated with higher BMI in overweight children until the age of 9 (4 years follow-up), corrected for actual BMI zscores at age 5 years. However, note that children of parents who accurately perceived their child's weight status had a higher actual BMI at age 5 years. Furthermore, children of parents who correctly perceived their child as being overweight showed a consistently higher BMI at different ages, compared to children of parents who underestimated their child's overweight. This probably made it easier for parents to correctly perceive their child's overweight. Nevertheless, even after correction for actual BMI z-scores, the association between accurate weight status perception and higher BMI at 
follow-up remained statistically significant. This would imply that parental awareness of the child's overweight as such is not protective against subsequent overweight development. This contradicts the findings of Kroke and colleagues (2006) who reported that maternal misperception about overweight children aged 6 months had an unfavourable effect on the child's weight development, compared to mothers who perceived their child's weight status correctly. However, as the researchers themselves acknowledged (Kroke et al., 2006), determination of actual weight status using BMI in children below the age of 2 years may be an inappropriate measure (Whitaker, Wright, Pepe, Seidel, \& Dietz, 1997), and the feeding mode (breastfeeding or bottle-feeding) had a differential effect on early body mass development (Gubbels, Thijs, Stafleu, Van Buuren, \& Kremers, 2011b). Also, their study included relatively small subsamples, impeding external validity.

Eighty-five percent of the parents of overweight children in our study underestimated their child's weight status. Similar percentages were found in previous studies, with $72-90 \%$ of the parents underestimating the weight status of their overweight child (Baughcum et al., 2000; De Hoog et al., 2012; De La, 2009; Oude Luttikhuis et al., 2010; Yao \& Hillemeier, 2012).

Several potential causes may underlie inaccurate parental weight status perception (Maynard et al., 2003). First, parents may not recognize their child as overweight; they just do not see it or do not have the skills to see it. Another cause may be that parents do not understand what overweight means. This is suggested by the findings of Jain et al. (2001) who performed qualitative interviews with low-income mothers to understand their perceptions of when a child is overweight. The authors found that these mothers did not accept the classifications used by health professionals to determine a child's overweight. Finally, parents may feel reluctant to admit that their child is overweight (Gerards, Dagnelie, Jansen, De Vries, \& Kremers, 2012a). Other studies reported that parental perception of their child's weight status was related to the mother's own overweight status, which may influence their norm regarding what is a normal weight (Chaparro et al., 2011; Mamun et al., 2008). Although a relatively large proportion of the parents in the current study were overweight or obese, we could not find such an association between parental BMI and the parent's perception of their child's weight status or the child's BMI change.

Predictors of accurate weight status perception, in both overweight and normal weight children aged 5 years, were the children's actual BMI z-score at 5 years and the children's BMI z-score at 2 years (both predictors being positively associated with accurate weight status perception). Other studies have also shown a positive association between actual cross-sectional BMI (z-scores) and weight status perception (Manios et al., 2009; Maynard et al., 2003), but we are the first to investigate the influence of prior $\mathrm{BMI}$ on the parental perception of previous body weight status. In normal weight 
children, high educational level of the father was an additional significant predictor of accurate perception. In line with this, previous studies (Baughcum et al., 2000; De Hoog et al., 2012; Manios et al., 2009) found that mothers with a low educational level were more likely to underestimate their child's weigh status. We did not find any evidence that other demographic variables are significant predictors of accurate weight perception, which appears to contradict what other studies have reported (Baughcum et al., 2000; De Hoog et al., 2012; De La, 2009; Manios et al., 2009; Maynard et al., 2003; Yao \& Hillemeier, 2012). An explanation for the discrepancies between studies may be the use of different instruments for measuring weight status perception. Some studies (including the current study) have used written questionnaires to assess parental perception of their child's weight status (Baughcum et al., 2000; De Hoog et al., 2012; Garrett Wright, 2011; Mamun et al., 2008; Maynard et al., 2003; Yao \& Hillemeier, 2012), whereas others have used an interview technique (Chaparro et al., 2011; Kroke et al., 2006; Manios et al., 2009), which may make it even more emotionally challenging for parents to admit that their child is overweight compared to an anonymous questionnaire (Polit \& Beck, 2008). Another possible cause of discrepancies between study results may be differences in general characteristics of the study populations between earlier studies and ours. The KOALA cohort is relatively healthy; e.g., $9 \%$ of the 5 -year-old children were overweight or obese, compared to $13 \%$ of the Dutch boys and $18 \%$ of the Dutch girls at the age of 5 (Schonbeck et al., 2011). The cohort also included relatively highly educated parents: $53 \%$ of the parents had a high education level, compared to $26-31 \%$ of the general Dutch population (Leufkens \& Souren, 2011). These specific general characteristics of the KOALA cohort may mean that the influence of several predictors was not detected, because of power issues.

In the present study, 5-year-old children whose parents underestimated their child's overweight status were generally only overweight at that particular measurement, whereas the children whose parents accurately perceived their child's overweight consistently had a higher average BMI over time. This indicates that children whose parents accurately perceive their weight differ in weight status from the children whose parents underestimate their weight status. We found a similar pattern for parents of normal weight children: children of parents, who correctly perceived their child as normal weight, had a consistently higher BMI compared to children of parents who underestimated their child as underweight. This implies that one should be very careful when examining parental perceptions of the child's weight status in only one cross-sectional weight measurement. It seems that parents can accurately take into account their child's weight history in assessing his or her weight status at one particular point in time. Alternatively, this phenomenon may be a statistical artefact, caused by measurement errors in either the child's body weight or parental weight status perception, or regression to the mean. 
Accurate weight status perception may be an important prerequisite for involving parents in childhood obesity interventions (Gerards et al., 2012a). In general, parents who are not aware that their child is overweight, will not feel the need to become involved in such an intervention. In one adolescent family-based intervention, for example, parental weight status perception proved an important predictor of treatment initiation (Dhingra, Brennan, \& Walkley, 2011). Nevertheless, accurate weight status perception alone is not sufficient. Parents may not have sufficient motivation, skills or parenting practices to manage their child's overweight, or they may even use counterproductive strategies, such as extreme restriction or overcontrolling of unhealthy energy balance-related behaviors, to address their child's overweight (Gubbels et al., 2011a). This may result in further BMI increases in the long run. Parents therefore need to be enabled to develop adequate skills to manage their child's overweight in family-based interventions (Gerards et al., 2012b; West et al., 2010b).

Some limitations should be taken into account when interpreting our results. Almost all data were self-reported by parents, which may have led to bias. However, the weight and height data at age 5 were partly measured by trained research assistants. We tested whether the self-report and measured data led to different results, using interaction terms in the models, and found no difference between the two measurement methods. This is line with the findings by Scholtens et al. (2007) who reported that parental reports of their child's actual weight and height are relatively valid. However, parents of overweight children tend to underreport their child's weight (Scholtens et al., 2007; Timmermans et al., 2013), indicating that even more parents underestimate their child's weight than we reported in the current study. Unfortunately, we were not able to conduct separate analyses of parental weight status perception for the group of obese children, as the small number of children in this subsample $(n=32)$ mean there was insufficient statistical power for such analyses. The final limitation regards the representativeness of the KOALA cohort, as already noted.

To our knowledge, this is the first study to examine parents' perceptions of their child's weight status in relation to longitudinal weight status development, both prior and subsequent to the assessment of parental perception. It would be valuable to further investigate the effect of underestimation on children's weight status, specifically with regard to causality and underlying mechanisms explaining the associations found in the current study. Future studies with a larger sample of overweight children should investigate what differences there are, within the group of 'consistently' overweight children, between parents who accurately estimate their child's weight status and those who underestimate it. 


\section{Conclusions}

Children of parents who accurately perceived their child's weight status had a higher BMI over time, making it easier for parents to correctly perceive their child's overweight. Mere awareness of the child's weight status as such might thus be not beneficial for subsequent BMI development. It would therefore appear appropriate to develop and test new interventions in which parents are taught skills for managing their children's weight-related behaviors. 



\section{CHAPTER 5}

\section{Parental self-efficacy in childhood overweight: validation of the Lifestyle Behavior Checklist in the Netherlands}

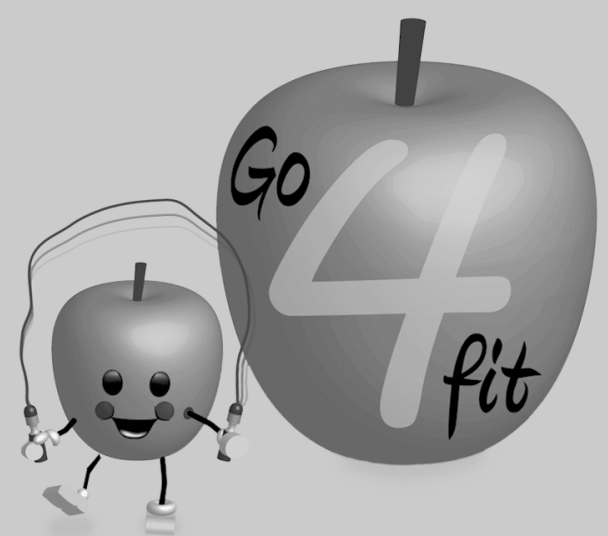

Published as: Gerards, S.M.P.L., Hummel, K., Dagnelie, P.C., De Vries, N.K. and Kremers, S.P.J. (2013). Parental self-efficacy in childhood overweight: validation of the Lifestyle Behavior Checklist in the Netherlands. International Journal of Behavioral Nutrition and Physical Activity, 10: 7 


\section{Abstract}

Background: Evaluating whether parental challenges and self-efficacy toward managing children's lifestyle behaviors are successfully addressed by interventions requires valid instruments. The Lifestyle Behavior Checklist (LBC) has recently been developed in the Australian context. It consists of two subscales: the Problem scale, which measures parental perceptions of children's behavioral problems related to overweight and obesity, and the Confidence scale, measuring parental self-efficacy in dealing with these problems. The aim of the current study was to systematically translate the questionnaire into Dutch and to evaluate its internal consistency, construct validity and test-retest reliability.

Methods: The LBC was systematically translated by four experts at Maastricht University. In total, 392 parents of 3- to 13-year-old children were invited to fill out two successive online questionnaires with a two-week interval. Of these, 273 parents responded to the first questionnaire (test, response rate $=69.6 \%$ ), and of the 202 who could be invited for the second questionnaire (retest), 100 responded (response rate = 49.5\%). We assessed the questionnaire's internal consistency (Cronbach's $\alpha$ ), construct validity (Spearman's Rho correlation tests, using the criterion measures: restrictiveness, nurturance, and psychological control), and test-retest reliability (Spearman's Rho correlation tests).

Results: Both scales had high internal consistency (Cronbach's $\alpha \geq 0.90)$. Spearman correlation coefficients indicated acceptable test-retest reliability for both the Problem scale $\left(r_{s}=0.74\right)$ and the Confidence scale $\left(r_{s}=0.70\right)$.

The LBC Problem scale was significantly correlated to all criterion scales (nurturance, restrictiveness, psychological control) in the hypothesized direction, and the LBC Confidence scale was significantly correlated with nurturance and psychological control in the hypothesized direction, but not with restrictiveness.

Conclusions: The Dutch translation of the LBC was found to be a reliable and reasonably valid questionnaire to measure parental perceptions of children's weightrelated problem behavior and the extent to which parents feel confident to manage these problems. 


\section{Background}

The prevalence of childhood overweight and obesity is steadily increasing worldwide (WHO, 2013). In the Netherlands, $13-15 \%$ of the Dutch children were overweight in 2009, a two- to three-fold increase relative to 1980 figures (Schonbeck et al., 2011). In addition, two percent of the children were obese, which is four to six times the prevalence in 1980. In response to this increase, an increasing number of interventions have been developed with the aim of preventing or treating overweight and obesity in children. A substantial number of these interventions are aimed at parents (Gerards et al., 2011; Golley et al., 2011; Kitzmann et al., 2010), who are important contributors to children's energy balance-related behaviors (i.e. food intake and physical activity behaviors that are primary determinants of weight gain (Kremers et al., 2005)) and weight status. Evaluating the effects of parenting interventions on relevant intermediate outcome measures is necessary to get insight in working mechanisms of interventions. However, for this purpose, validated instruments are required.

Parenting can be a challenging job. Parents generally receive little preparation apart from having been parented themselves, so most parents learn by trial and error (Sanders et al., 2003). In identifying parenting-related behaviors, two levels are often distinguished (Sleddens et al., 2011): specific parenting practices and general parenting styles. Specific parenting practices are behaviors that relate to a specific domain (e.g. nutrition or physical activity). Examples of specific parenting practices are rules about having breakfast or controlling the availability of fruit at home. General parenting styles reflect the emotional climate in which behavior-specific parenting takes place, which determines the context in which parent-child interactions occur (Darling \& Steinberg, 1993). Examples of parenting styles include responsiveness (extend to which parents are aware of their children's feelings, problems and difficulties and the way they respond in a supportive way), or demandingness (controlling children's behaviors). In the nutrition and parenting literature, both levels of parenting have been shown to be of importance in explaining and predicting children's energy balancerelated behaviors (Sleddens et al., 2011). In addition, parenting behaviors that may contribute to a child's positive energy balance seem to be positively associated with parental weight (or BMI) (Gubbels et al., 2011a; Montgomery, Jackson, Kelly, \& Reilly, 2006).

In contrast to parenting practices and styles, the construct of weight-related parenting self-efficacy has been largely neglected in the research on food and activity parenting (Nelson \& Davis, 2013; Smith et al., 2010). According to Bandura (1995), selfefficacy is 'a person's belief in his capabilities to organize and execute the course of action required to manage prospective situations'. In the general parenting literature, self-efficacy is recognized as an important determinant of parenting behaviors 
(Coleman \& Karraker, 2000; Jones \& Prinz, 2005). As acknowledged by the few prior studies in this area (Nelson \& Davis, 2013; Smith et al., 2010; West \& Sanders, 2009), low parental self-efficacy may be a barrier for parents trying to change their children's nutrition and physical activity behaviors. Compared to parents with healthy children, parents of overweight and obese children may face additional challenges in the upbringing of their children, including being worried about stigmatization of their child, wanting to protect their child from stigmatization, feeling ambivalent about setting limits for their child, uncertainty about being a good parent, and uncertainty about, on the one hand, accepting the child as he/she is and, on the other hand, feeling responsible for their child's health (Haugstvedt, Graff Iversen, Bechensteen, \& Hallberg, 2011). Mothers of overweight and obese children have reported frustration as a result of their children's unwillingness to eat a healthy diet and be physically active (Jackson, Wilkes, \& McDonald, 2007). This overweight-specific parental self-efficacy differs in nature from behavioral practices and styles, but it seems to be an essential additional component for parents to succeed in raising their children to become healthy adults.

As a first start towards developing a parenting intervention, it is valuable to identify initial parental challenges in managing children's lifestyle behavior and to assess parental self-efficacy. It is also useful to investigate whether parental confidence and skills regarding weight-related challenges are addressed by interventions. In view of the lack of a specific instrument to measure weight-related parental self-efficacy, West \& Sanders (2005) developed the Lifestyle Behavior Checklist (LBC), a tool to measure parental perceptions of their children's behavioral problems with overweight and obesity, and parents' self-efficacy in dealing with these behaviors.

West and colleagues performed two studies in Australia to test the validity of the LBC (West, Morawska, \& Joughin, 2010a; West \& Sanders, 2009). They tested its content validity by determining whether the LBC could be used to distinguish between families with and families without obese children. Parents of children with a healthy weight reported lower levels of lifestyle behavior problems and higher levels of parental self-efficacy. Furthermore, the construct validity of the LBC questionnaire was assessed by using general parenting measures as criterion measures; both scales were significantly correlated with the measures of general parenting, indicating that general parenting skills are to some extent reflected in parental self-efficacy in childhood overweight. In addition, evidence suggests that the LBC scales are responsive to change following a parenting intervention (West et al., 2010b).

The aim of the current study was to test psychometric properties of the LBC questionnaire in the Dutch context. We translated the LBC questionnaire from English into Dutch. We also tested the construct validity of the Dutch version of the LBC using general parenting style measures as criterion variables. Finally, we determined the test- 
retest reliability of the Dutch version of the questionnaire, by sending participants the questionnaire twice with a two-week interval.

\section{Methods}

\section{Overview of procedures and participants}

Potential participants were invited to participate via an online survey panel (Thesistools, The Hague). This panel consists of participants who receive an invitation to participate in a survey once a month. They do not receive a reward for participation. Respondents were included if they were parents of children aged 3-13 years, and living in the Netherlands. Two weeks after they filled out the first questionnaire, a second questionnaire (retest; LBC only) was sent to respondents who had provided their email addresses. Of the 392 participants who were exposed to the questionnaire as intended, 273 were included in the analyses. Reasons for exclusion were: not being a parent of a 3- to 13 -year-old child ( $N=33)$, more than $10 \%$ of the answers missing $(N=45)$, or being a parent of an underweight child $\left(<5^{\text {th }}\right.$ percentile) $(N=41)$. Of the 273 participants included in the analysis, $202(74.0 \%)$ gave their permission to be invited for the second questionnaire by email. Of these, 100 responded to the second questionnaire (response rate for second questionnaire $=49.5 \%$ ). These numbers are depicted in the flowchart in Figure 5.1.

\section{Measures}

The Lifestyle Behavior Checklist

The LBC is a 25 -item questionnaire which assesses parental perceptions of the extent of behavior problems of overweight and obese children and parental confidence about managing these problems (West \& Sanders, 2009). The questionnaire assesses a list of 25 child problem behaviors related to eating (e.g., eats too much, argues about food), activity (e.g., watches too much television, refuses to do physical activity), and overweight (e.g., complains about being overweight, complains about not fitting into clothes). The questionnaire consists of a Problem scale and a Confidence scale. The Problem scale measures the extent to which parents perceive each of the 25 behaviors as a problem for them with their child, on a 7-point scale from 1 (not at all) to 7 (very much). The Confidence scale measures the extent to which parents feel confident about managing each of the behaviors, on a 10-point scale from 1 (certain I cannot do it) to 10 (certain I can do it). The scores on the Problem scale and the Confidence scale are combined into two sum scores, ranging from 26 to 182 and from 26 to 260, respectively. 


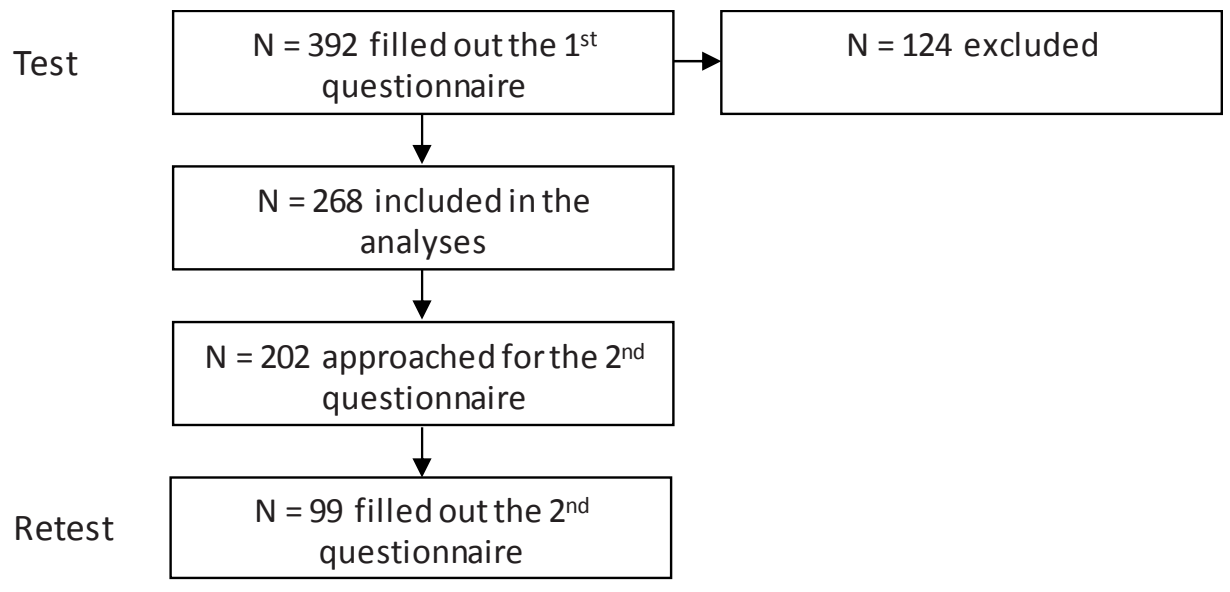

Figure 5.1: Flow diagram of participants

\section{Translation procedure}

The LBC was translated into Dutch by four experts at Maastricht University (the Netherlands) who are also authors of this manuscript (SMPLG, KH, PCD, SPJK). The translation procedure was as follows. First, all translators independently translated the questionnaire. Inconsistencies were then discussed in a plenary session until consensus was reached and a provisional version of the questionnaire was developed. This provisional questionnaire was pretested among 2 students and 3 parents who were part of the target population. The pretest was based on cognitive interviewing, i.e. using verbal probing techniques (Willis, 1999). Subsequently, another meeting between the experts took place to discuss the results of the pretest. In case of uncertainties, we contacted the developer of the questionnaire (F. West). All translators approved the final translation.

\section{Demographics}

Participants were asked to specify their relation to the child (biological mother, biological father, stepmother, stepfather, or other), their educational level, and the educational level of their partner, their employment status, and the employment status of their partner. Educational level was categorized into three different levels, i.e. low (primary school or lower secondary vocational education), medium (junior general secondary education, senior secondary vocational education, senior general secondary education or pre-university education), and high (higher professional education or university education). Employment status was divided into two categories, viz. employed or not. 
Additional questions concerned the date of birth, height and weight of both parents. Items regarding the child included date of birth (to determine age), height and weight, gender and number of siblings. Height and weight of the parents and children were used to calculate BMI (weight $(\mathrm{kg}) /$ height $(\mathrm{m}))^{2}$. Children's BMI was recoded into BMI z-scores compared to the 1997 national reference population (Fourth Dutch National Growth Study) (Fredriks et al., 2000). Weight status was classified into healthy weight $\left(5^{\text {th }}-84^{\text {th }}\right.$ percentile $)$, overweight $\left(85^{\text {th }}-94^{\text {th }}\right.$ percentile $)$ or obesity ( $\geq 95^{\text {th }}$ percentile) (Barlow, 2007).

\section{General parenting}

Items from the Child Rearing Practices Report scale as well as items from the Psychological Control scale, both validated Dutch versions, were included in the questionnaire as criterion validation scales to assess three theory-based general parenting dimensions: restrictiveness, nurturance and psychological control (Barber, 1996; Skinner, Johnson, \& Snyder, 2005). The Child Rearing Practices Report (CRPR) in its original form consists of 91 items to assess parents' child-rearing attitudes, values, behaviors and goals (Block, 1965). The CRPR has been shown to be a valid instrument for assessing child-rearing dimensions in the Dutch population (Dekovic, 1989). In the current study, we included 35 CRPR items, based on validation studies of shorter versions of the questionnaire (Dekovic, 1989; Dekovic, Janssens, \& Gerris, 1991). The CRPR items can be divided into two scales: a restrictiveness scale and a nurturance scale. Restrictiveness items ( $n=18$, Cronbach's $\alpha=0.80$ ) are characterized by a high degree of control, narrow limit setting, and endorsing of strict rules, requirements and restrictions (e.g. 'I try to keep my children away from children or families whose ideas or values are different from our own'). The items on the nurturance scale ( $n=17$, Cronbach's $\alpha=0.78$ ) reflect parents' willingness to listen to their children and share feelings and experiences with them, parents' responsiveness to their children's needs, and the extent to which parents show affection and acceptance (e.g. 'I respect my child's opinion and encourage him to express it').

In addition, eight psychological control items (Cronbach's $\alpha=0.72$ ) from the Psychological Control Scale (Barber, 1996) were added in the questionnaire to include a relevant third parenting dimension (Barber, 1996). Psychological control is defined as 'parental behaviors (such as guilt induction, love withdrawal or contingent love, instilling anxiety, and invalidation of the child's perspective) that are intrusive and manipulative to children's thoughts, feelings, and attachments to parents' (Barber, 1996). An example item is 'I am less friendly with my child when he/she does not see things my way'. 


\section{Data analysis}

SPSS 17.0 was used for the analyses. Descriptive statistics were used to calculate means and standard deviations of the quantitative continuous variables, and to calculate percentages of the categorical data. To determine the internal consistency of the scales, we calculated Cronbach's $\alpha$. Spearman's Rho correlation tests were used to determine test-retest reliability.

Group means (healthy weight vs. overweight children) on the individual LBC items and the LBC scale scores were compared using one-way ANOVA. We applied ANOVA tests for the LBC scales scores with corrections for parenting constructs and demographic variables (educational status of both parents, employment status of both parents, maternal and paternal age, age of the child, BMI of both parents and BMI z-score of the child).

Construct validity between the LBC scales and the parenting styles scales was assessed using bivariate correlations (Spearman's Rho correlation tests) and partial correlations, corrected for the demographics. We used the magnitude of the relationship ('effect size') ( $r$ and partial $r$ ) as a source of information. Interpretation of the strength of the effect size was based on Cohen's descriptive guidelines (Cohen, 1988). A correlation higher than or equal to 0.50 was regarded as a large effect size, correlations between 0.30 and 0.50 as medium effect size, and a correlation higher than or equal to 0.10 as a small effect size. With regard to the partial $r$, a small effect size was defined as one larger than or equal to 0.02 , a medium effect as larger than 0.15 , and a large effect size as larger than or equal to 0.35 .

\section{Results}

\section{Characteristics of the participants}

Characteristics of the test and retest population are summarized in Table 5.1. In most cases, it was the biological mother of the child who filled out the questionnaire. Most parents had a high educational level ( $64.8 \%$ of mothers and $59.7 \%$ of fathers) and were employed (79.1\% of mothers and $90.5 \%$ of fathers). About one third of the mothers were overweight, and about $11.0 \%$ were obese. Half of the fathers were overweight or obese. Regarding the weight status of the children, $11.4 \%$ of the test sample was overweight or obese, compared to $13.0 \%$ of the retest sample. We tested whether we could predict drop-out with respect to the test-retest samples using demographics. No statistically significant predictors were found, indicating non-selective drop-out. 
Table 5.1: Characteristics of the samples

\begin{tabular}{|c|c|c|c|c|}
\hline Variable & \multicolumn{2}{|c|}{ Test sample $(N=273)$} & \multicolumn{2}{|c|}{ Retest sample $(N=100)$} \\
\hline Continuous & \multicolumn{2}{|c|}{ Mean (SD) } & \multicolumn{2}{|c|}{ Mean (SD) } \\
\hline \multicolumn{5}{|l|}{ Age (years) } \\
\hline Mother & \multicolumn{2}{|c|}{40.35 (7.01) } & \multicolumn{2}{|c|}{$41.70(6.75)$} \\
\hline Father & \multicolumn{2}{|c|}{$42.84(7.33)$} & \multicolumn{2}{|c|}{$44.20(7.25)$} \\
\hline Child & \multicolumn{2}{|c|}{$7.88(2.73)$} & \multicolumn{2}{|c|}{$8.08(2.78)$} \\
\hline Number of siblings & \multicolumn{2}{|c|}{$2.30(1.04)$} & \multicolumn{2}{|c|}{$2.25(1.03)$} \\
\hline \multicolumn{5}{|l|}{ BMI } \\
\hline Mother & \multicolumn{2}{|c|}{$24.93(4.18)$} & \multicolumn{2}{|c|}{$25.12(4.34)$} \\
\hline Father & \multicolumn{2}{|c|}{$25.33(3.34)$} & \multicolumn{2}{|c|}{$25.25(3.43)$} \\
\hline Child & \multicolumn{2}{|c|}{$16.31(2.24)$} & \multicolumn{2}{|c|}{$16.51(2.18)$} \\
\hline BMI z-score child & \multicolumn{2}{|c|}{$-0.08(1.00)$} & \multicolumn{2}{|c|}{$-0.03(0.94)$} \\
\hline Categorical & $\mathrm{N}$ & $\%$ & $\mathrm{~N}$ & $\%$ \\
\hline \multicolumn{5}{|l|}{ Child sex } \\
\hline Male & 140 & 51.3 & 50 & 50.0 \\
\hline Female & 133 & 48.7 & 50 & 50.0 \\
\hline \multicolumn{5}{|l|}{ Relation to child } \\
\hline Biological mother & 209 & 76.6 & 78 & 78.0 \\
\hline Biological father & 56 & 20.5 & 19 & 19.0 \\
\hline Stepmother & 2 & 0.7 & 1 & 1.0 \\
\hline Stepfather & 0 & 0 & 0 & 0 \\
\hline Other & 6 & 2.2 & 2 & 2.0 \\
\hline Mother's education & & & & \\
\hline Low & 4 & 1.5 & 1 & 1.0 \\
\hline Medium & 86 & 31.5 & 27 & 27.0 \\
\hline High & 177 & 64.8 & 70 & 70.0 \\
\hline Missing & 6 & 2.2 & 2 & 2.0 \\
\hline Father's education & & & & \\
\hline Low & 16 & 5.9 & 5 & 5.0 \\
\hline Medium & 88 & 32.2 & 27 & 27.0 \\
\hline High & 163 & 59.7 & 66 & 66.0 \\
\hline Missing & 6 & 2.2 & 2 & 2.0 \\
\hline Mother employed & & & & \\
\hline No & 51 & 18.7 & 18 & 18.0 \\
\hline Yes & 216 & 79.1 & 80 & 80.0 \\
\hline Missing & 6 & 2.2 & 2 & 2.0 \\
\hline Father employed & & & & \\
\hline No & 20 & 7.3 & 9 & 9.0 \\
\hline Yes & 247 & 90.5 & 89 & 89.0 \\
\hline Missing & 6 & 2.2 & 2 & 2.0 \\
\hline Mother's weight cate & & & & \\
\hline Underweight & 3 & 1.1 & 1 & 1.0 \\
\hline Healthy weight & 150 & 54.9 & 57 & 57.0 \\
\hline Overweight & 90 & 33.0 & 31 & 31.0 \\
\hline Obesity & 30 & 11.0 & 11 & 11.0 \\
\hline
\end{tabular}




\begin{tabular}{|c|c|c|c|c|}
\hline \multirow{2}{*}{$\begin{array}{l}\text { Variable } \\
\text { Categorical }\end{array}$} & \multicolumn{2}{|c|}{ Test sample $(N=273)$} & \multicolumn{2}{|c|}{ Retest sample $(N=100)$} \\
\hline & $\mathrm{N}$ & $\%$ & $\mathrm{~N}$ & $\%$ \\
\hline \multicolumn{5}{|c|}{ Father's weight category } \\
\hline Underweight & 0 & 0 & 0 & 0 \\
\hline Healthy weight & 139 & 50.9 & 47 & 47.0 \\
\hline Overweight & 110 & 40.3 & 47 & 47.0 \\
\hline Obesity & 24 & 8.8 & 6 & 6.0 \\
\hline \multicolumn{5}{|c|}{ Child's weight category } \\
\hline Healthy weight & 242 & 88.6 & 87 & 87.0 \\
\hline Overweight & 16 & 5.9 & 6 & 6.0 \\
\hline Obesity & 15 & 5.5 & 7 & 7.0 \\
\hline
\end{tabular}

\section{Reliability}

Means and standard deviations of the Problem and Confidence scales are listed in Table 5.2. Both the Problem scale (Cronbach's $\alpha$ test $=0.92$, retest $=0.91$ ) and the Confidence scale (Cronbach's $\alpha$ test $=0.98$, retest $=0.90$ ) had high internal consistency. Spearman correlation coefficients, to determine test-retest reliability, were acceptable for the Problem scale $\left(r_{s}=0.74, p<0.001\right)$, as well as the Confidence scale $\left(r_{s}=0.70, p\right.$ $<0.001)$.

Table 5.2: Mean scores of study sample on parenting scales

\begin{tabular}{lllll}
\hline & Range & $\begin{array}{l}\text { Test sample }(N=273) \\
\text { Mean }(S D)\end{array}$ & $\begin{array}{l}\text { Retest sample }(N=100) \\
\text { Mean }(S D)\end{array}$ \\
\cline { 3 - 5 } & & & Test scores & Retest scores \\
\hline LBC Problem scale & $26-182$ & $39.12(14.00)$ & $38.15(10.27)$ & $39.18(12.63)$ \\
LBC Confidence scale & $26-260$ & $208.14(32.85)$ & $210.37(28.63)$ & $205.57(28.78)$ \\
Nurturance & $1-5$ & $4.51(0.33)$ & $4.53(0.28)$ & - \\
Restrictiveness & $1-5$ & $2.48(0.47)$ & $2.42(0.47)$ & - \\
Psychological control & $1-5$ & $1.79(0.53)$ & $1.72(0.50)$ & - \\
\hline
\end{tabular}




\section{Group differences}

The scores on individual items of the Problem scale and the Confidence scale were compared between parents of healthy weight and overweight (including obese) children (see Table 5.3). With regard to the Problem scale items, parents of overweight children scored significantly higher on 14 of the 25 items compared to parents of healthy weight children. On four confidence items, parents of overweight children scored significantly lower than parents of normal weight children. Other Confidence scale items did not significantly differ between the parents of non-overweight and those of overweight children.

The ANOVA analyses of the LBC scale scores revealed a significant difference in scores onto the Problem scale between children with and without overweight ( $F(1$, $246)=16.94, p<0.001)$. Parents in the healthy weight group scored significantly lower on the Problem scale $(M=37.83, S D=13.27)$, compared to those in the overweight group ( $M=49.21, S D=15.57$ ). The group effect on the Problem scale remained significant after correcting for the covariates (parenting constructs and demographic variables), $(F(1,237)=11.48, p=0.001)$. There was no group effect for the Confidence scale $(F(1,246)=1.47, p=0.227)$ : the parents of healthy weight children $(M=209.06$, $\mathrm{SD}=33.01$ ) did not score significantly higher on the Confidence scale than those of the overweight children ( $M=200.66, S D=31.07)$. The effect of the group on the Confidence scale, corrected for covariates (parenting constructs and demographic variables), was somewhat higher than the uncorrected effect (viz. $F(1,237)=1.49, p=$ 0.224), but still not statistically significant. 


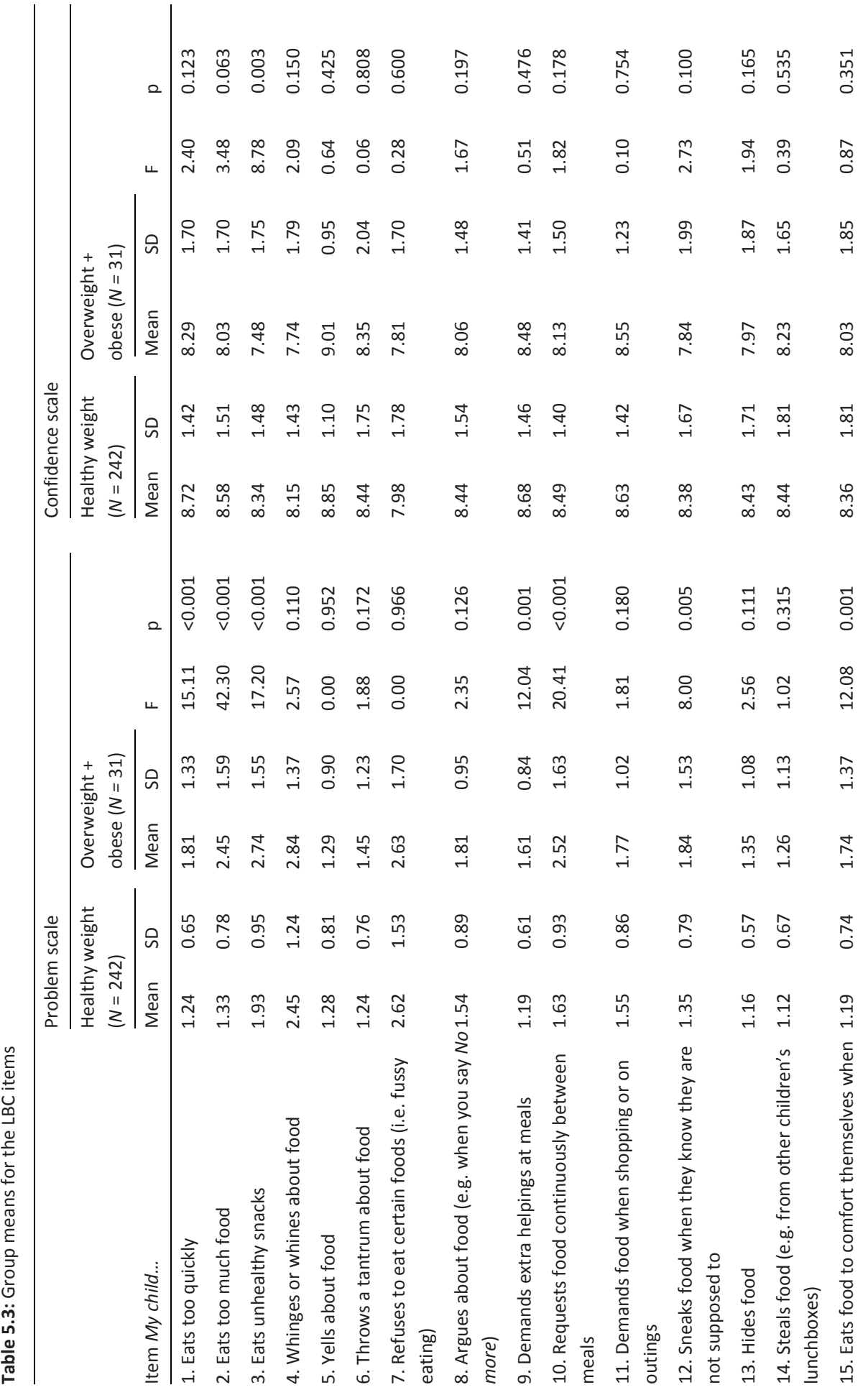


PARENTAL SELF-EFFICACY IN CHILDHOOD OVERWEIGHT I 121

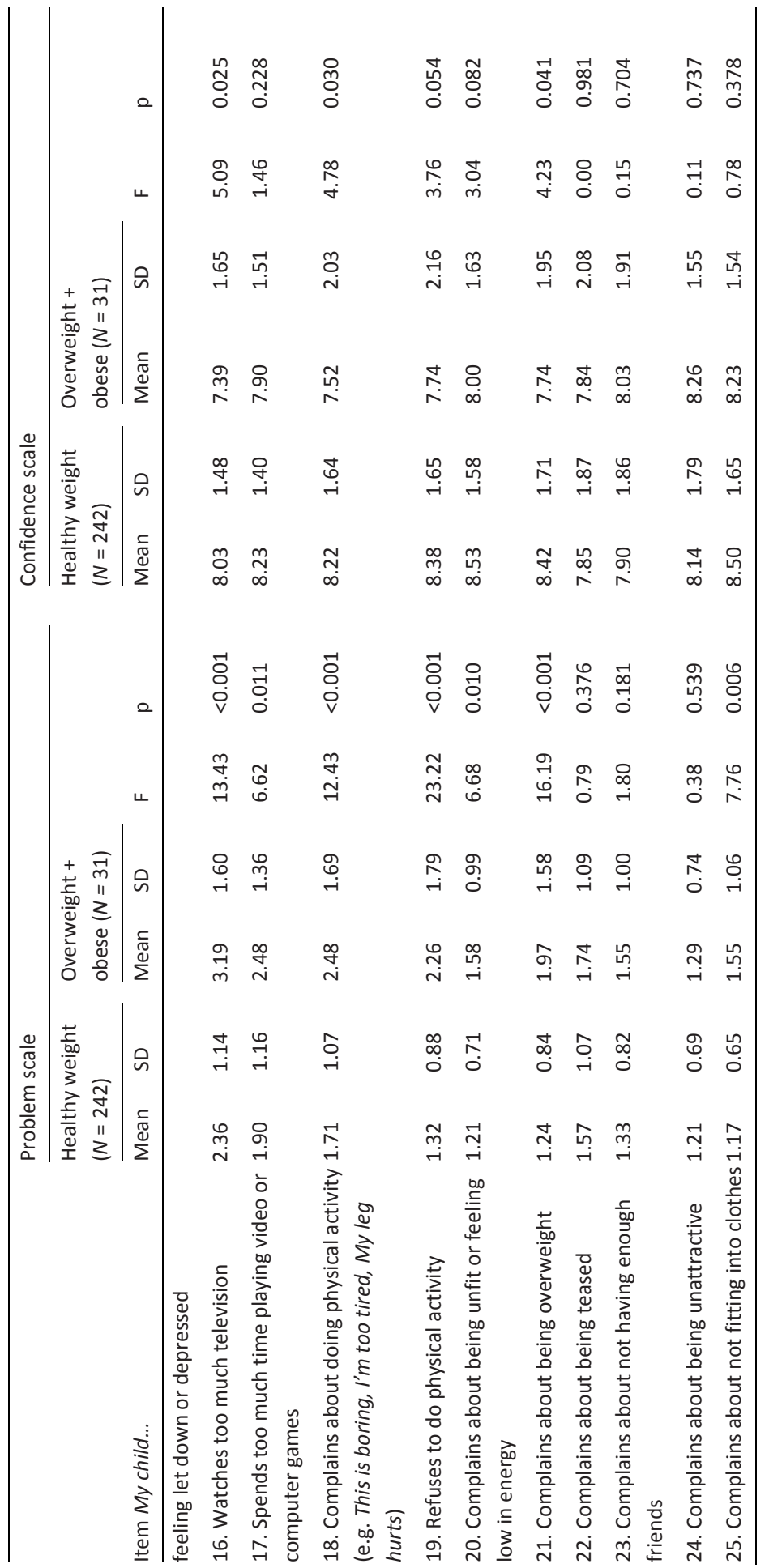




\section{Construct validity}

The LBC scales were tested for construct validity using the parenting style dimensions and weight measures of both parents and children. Results are shown in Table 5.4. We found significant correlations (unadjusted) between the LBC Problem scale and all criterion scales. The Problem scale was negatively correlated to nurturance (positive parenting dimension), and positively correlated to restrictiveness (negative parenting dimension), psychological control (negative parenting dimension) and the weight status of the child and both parents. The effect sizes of these correlations were small. After correction for demographics and parenting constructs, the Problem scale was significantly correlated to nurturance, BMI z-score of the child, and BMI scores of both parents.

Furthermore, we found an interaction effect of child weight status and the LBC Problem scale on nurturance. This means that the correlation between nurturance and the LBC Problem scale was different for parents of healthy weight children (unadjusted $r_{s}=-0.20$; adjusted $r=-0.14$ ) than for parents overweight and obese children (unadjusted $r_{\mathrm{s}}=-0.54$; adjusted $r=-0.48$ ).

The LBC Confidence scale was positively correlated to nurturance and negatively correlated to psychological control. Again, both effect sizes were small. The Confidence scale did not correlate with the other criterion measures. The adjusted scores of the Confidence scale were positively correlated to restrictiveness and negatively correlated with psychological control. All adjusted effect sizes were small.

Table 5.4: Correlation coefficients between the LBC scales and the criterion measures

\begin{tabular}{|c|c|c|c|c|}
\hline \multirow{2}{*}{$\begin{array}{l}\text { LBC scale } \\
\text { Criterion scales }\end{array}$} & \multicolumn{2}{|c|}{ LBC Problem scale } & \multicolumn{2}{|c|}{ LBC Confidence scale } \\
\hline & Unadjusted $\left(r_{s}\right)$ & $\begin{array}{l}\text { Adjusted for } \\
\text { parenting and } \\
\text { demographics }^{a}\end{array}$ & Unadjusted $\left(r_{s}\right)$ & $\begin{array}{l}\text { Adjusted for } \\
\text { parenting and } \\
\text { demographics }^{\text {a }}\end{array}$ \\
\hline Nurturance & $-0.23^{* *}$ & $-0.20 * *$ & $0.14^{*}$ & 0.04 \\
\hline Restrictiveness & $0.14^{*}$ & 0.06 & 0.04 & $0.13 *$ \\
\hline Psychological control & $0.19 * *$ & 0.05 & $-0.22^{* *}$ & $-0.18^{* *}$ \\
\hline BMI z-score of child & $0.21^{* *}$ & $0.21 * *$ & -0.02 & -0.06 \\
\hline BMI of mother & $0.23 * *$ & $0.18^{* *}$ & -0.06 & -0.06 \\
\hline BMI of father & $0.14^{*}$ & $0.16^{* *}$ & -0.02 & -0.03 \\
\hline
\end{tabular}

Note: ${ }^{*} p<0.05,{ }^{* *} p<0.01 ; r \geq 0.10$ small effect size, $r \geq 0.30$ medium effect size, $r \geq 0.50$ large effect size; partial $r \geq 0.20$ small effect size, partial $r \geq 0.15$ medium effect size, partial $r \geq 0.35$ large effect size; ${ }^{a}$ Adjusted for the other two parenting dimensions and demographics including educational status of both parents, employment status of both parents, maternal and paternal age, age of the child, BMI z-score of the child, and $\mathrm{BMI}$ of the father and the mother. 


\section{Discussion}

There is a need for instruments to assess parents' problems regarding their children's overweight and parents' self-efficacy in managing these problems. The Lifestyle Behavior Checklist (LBC) could be a valuable addition to existing parenting instruments, especially if it can be shown to have good psychometric properties. The present study was the first to validate the LBC outside Australia (cross-national validation). In the Dutch context, the translated LBC was found to be a reliable and reasonably valid questionnaire to measure weight-related parental self-efficacy. However, the questionnaire appeared to be somewhat less valid in our sample than in the Australian validation studies.

The LBC Problem scale was significantly negatively correlated to the general parenting construct nurturance (positive parenting dimension), and positively correlated to restrictiveness (negative parenting dimension) and psychological control (negative parenting dimension). The Confidence scale was negatively correlated to psychological control, and positively correlated to nurturance. These small but significant correlations indicated that the parenting constructs were related but not identical to the Problem and Confidence scales. The correlations were in the hypothesized direction. In the Australian validation study (West \& Sanders, 2009), the LBC Confidence scale was found to correlate moderately well with the Parenting Scale by Arnold (Arnold et al., 1993), which measures ineffective parenting (including permissive or authoritarian discipline).

Internal consistency of both scales was relatively high in both the test and retest. Correlation coefficients indicated relatively high test-retest reliability. These were comparable to the scores reported by the Australian validation study $\left(r_{s}=0.87\right.$ for the Problem scale, $r_{s}=0.66$ for the Confidence scale).

The Confidence scale seemed to be less sensitive than the Problem scale as regards detecting differences between parents of healthy weight children and parents of overweight children. In the Australian validation study (West \& Sanders, 2009), statistically significant differences between groups with different weight status were found for both scales. However, that study did not compare parents of overweight children with those of healthy weight children, but compared parents of healthy weight children with those of obese children. This difference in samples probably explains why the mean scores of parents of Australian obese children on all Problem scale items were substantially higher than the scores of the parents of overweight children in our sample. Scores on Confidence scale items were substantially lower among parents of obese children in the Australian study.

We found an interaction effect between child weight status and the Problem scale for nurturance. For parents of overweight and obese children, there was a high nega- 
tive correlation between the LBC Problem scale and nurturance, whereas a small negative correlation between the LBC Problem scale and nurturance was found for the parents of healthy weight children. We already knew from an earlier review (Sleddens et al., 2011) that the parenting dimension nurturance was positively related to overweight-preventing behaviors. Parents of overweight children may have a different parenting style than those of healthy weight children. The finding in the current study that nurturance by parents of overweight children is strongly negatively correlated to children's weight-related problem behaviors confirms the protecting influence of nurturance.

The LBC includes 15 items related to dietary behavior, while only 4 items are related to physical activity or sedentary behavior and 6 items are related to the child's overweight. Although an increasing number of studies have shown the importance of sedentary behavior in determining the development of overweight and obesity (Owen, Healy, Matthews, \& Dunstan, 2010), it is conceivable that the relatively high proportion of diet-related items is in line with the actual everyday concerns of parents. Parents may indeed have more concerns about feeding their child (Pagnini, Wilkenfeld, King, Booth, \& Booth, 2007), whereas they may not have too many concerns about their child watching too much television (He, Irwin, Sangster Bouck, Tucker, \& Pollett, 2005) or not being physically active. Earlier studies even found that parents often do not know that watching too much television is related to the development obesity (Bolling, Crosby, Boles, \& Stark, 2009; He et al., 2005).

The LBC may also serve as a basis for an intervention or recruitment. It can be an important instrument to map parental problems, as it may also provide us with an opportunity to make parents aware of possible problems regarding to their children's overweight. We know that the programs aimed at the prevention of obesity often struggle with recruitment problems (Gerards et al., 2012a; Rice, Thombs, Leach, \& Rehm, 2008). However, when parents themselves recognize their child's overweight problems, they may be more willing to take action and participate in prevention programs.

Some strong and weak points of the current study should be acknowledged. A strong point of the current study was the quality of the translation process. Four experts independently translated the questionnaire and a qualitative pretest was used to optimize the translation. However, we did not back-translate the questionnaire, which could have had additional value to the translation process. We also evaluated testretest reliability of the LBC questionnaire. Another strong point was the relatively large sample we were able to include in the current study, making it more likely that the results can be generalized to a larger population. We recruited participants via an internet-based survey, which is known for its access to hidden populations (Crosby, DiClemente, \& Salazar, 2006). Nevertheless, several groups of people were un- 
derrepresented compared to the general Dutch population, as parents with a low educational level, parents with overweight and obesity, and parents of children with overweight and obesity were somewhat underrepresented. The present study lacked a test of other types of validity (e.g., discriminant validity), implying that we only partly showed evidence for the construct validity of the scale. Please note evidence for discriminant validity of the LBC has been provided in a previous study (West \& Sanders, 2009). It should also be noted that the response on the retest was relatively low, limiting external validity of the study. However, we tested whether drop-out was selective, which was not the case. Furthermore, weight and height measures were self-reported which may be a reason for the apparent lower child's weight status in our sample, compared to the Dutch population (Schonbeck et al., 2011).

We recommend that experts who develop and evaluate interventions to prevent and treat childhood obesity should also make use of measures of parents' self-efficacy in managing their child's energy balance-related behaviors, to assess changes in parental perceptions of their child's weight-related problems. The LBC can be a reliable and valid instrument to assess these intermediate intervention outcomes.

\section{Conclusions}

The Dutch translation of the Lifestyle Behavior Checklist seems to be a reliable and, reasonably valid questionnaire to measure parents' perception of their children's weight-related problem behavior and the extent to which parents feel confident about managing these problems. 



\section{CHAPTER 6}

\section{Barriers to successful recruitment of parents of overweight children for an obesity prevention intervention:}

a qualitative study among youth health care professionals

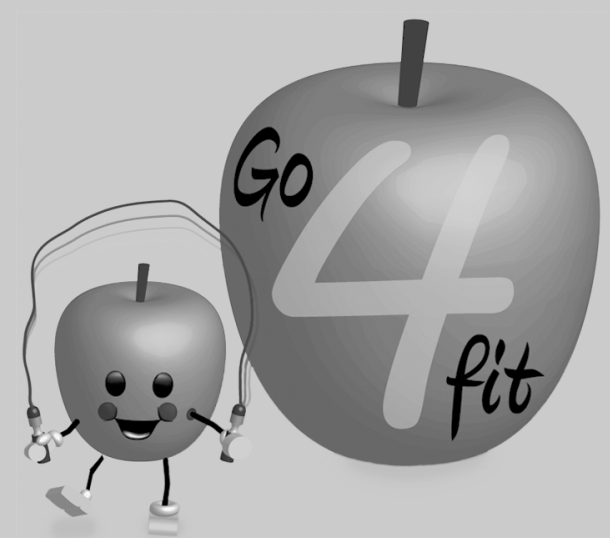

Published as: Gerards, S.M.P.L., Dagnelie, P.C., Jansen, M.W.J., De Vries, N.K., and Kremers, S.P.J. (2012). Barriers to successful recruitment of parents of overweight children for an obesity prevention intervention: a qualitative study among health care professionals. BMC Family Practice, 13: 37 


\section{Abstract}

Background: The recruitment of participants for childhood overweight and obesity prevention interventions can be challenging. The goal of this study was to identify barriers that Dutch youth health care (YHC) professionals perceive when referring parents of overweight children to an obesity prevention intervention.

Methods: Sixteen YHC professionals (nurses, physicians and management staff) from eleven child health clinics participated in semi-structured interviews. An intervention implementation model was used as the framework for conducting, analyzing and interpreting the interviews.

Results: All YHC professionals were concerned about childhood obesity and perceived prevention of overweight and obesity as an important task of the YHC organization. In terms of frequency and perceived impact, the most important impeding factors for referring parents of overweight children to an intervention were denial of the overweight problem by parents and their resistance towards discussing weight issues. A few YHC professionals indicated that their communication skills in discussing weight issues could be improved, and some professionals mentioned that they had low selfefficacy in raising this topic.

Conclusions: We consider it important that YHC professionals receive more training to increase their self-efficacy and skills in motivating parents of overweight children to participate in obesity prevention interventions. Furthermore, parental awareness towards their child's overweight should be addressed in future studies. 


\section{Background}

Childhood overweight and obesity are growing problems worldwide (Wang \& Lobstein, 2006). A key approach to preventing these problems and their adverse short- and longterm health consequences (Freedman et al., 2005; Singh et al., 2008) is the development and evaluation of practice- and theory-based health promotion interventions (Barkham \& Mellor Clark, 2003). Efficacy studies have shown that childhood obesity treatment and prevention programmes can be effective (Bluford et al., 2007; Doak et al., 2006; Kitzmann et al., 2010). The public health impact of these interventions, however, strongly depends on the proportion of the target group that is exposed to the intervention (Doak et al., 2006; Glasgow, Vogt, \& Boles, 1999).

One of the challenging aspects of implementing interventions to prevent or treat childhood overweight and obesity is the recruitment of participants (Rice et al., 2008; Warren et al., 2007), which is often considerably more difficult than expected (Warren et al., 2007). Recruitment may be particularly challenging when one or more of the other family members have to be engaged in the intervention programme. But even if only the children and not their parents participate in the intervention, the parents may be the largest obstacle to recruiting children for participation in weight-management programmes (Rice et al., 2008).

A general distinction can be made between active and passive recruitment methods (Lee et al., 1997). Active methods (or interpersonal channels) are methods in which researchers identify and approach potential participants (e.g., by phone, by mail or in person), whereas in passive methods subjects have to identify themselves as potential participants after exposure to, for instance, mass media channels, flyers and posters. A study comparing active and passive recruitment found that active recruitment, i.e. paediatrician referral and direct mail, produced the highest inclusion rate (Raynor et al., 2009), but a variety of obstacles to the active recruitment of children and their parents have been reported (Finne, Reinehr, Schaefer, Winkel, \& Kolip, 2009).

In the Netherlands, youth health care ( $\mathrm{YHC}$ ) is a unique system of preventive health care for all children aged 0-19 years (Verloove-Vanhorick et al., 2002). YHC professionals (physicians and nurses) systematically monitor the physical, psychological, social and cognitive health of children and advise parents and children on achieving a healthy development for the child in these respects. YHC professionals also signal possible health problems such as growth impairment, depression, aggression and overweight. If necessary, the YHC organization offers effective support or refers children to other health care facilities (Verloove-Vanhorick et al., 2002). The YHC service is offered by the government free of charge, and participation is voluntary. Annually, more than $90 \%$ of the 0-4 year old children are reached (e.g., in 2009, almost all 0-year-old chil- 
dren were reached and approximately $80 \%$ of the 4-year-old children) (Van Wieringen \& Van Wieren, 2008). This high level of reach makes the YHC service, in theory, an optimal setting to actively recruit children and their parents for health promotion programmes. YHC professionals are a potential gateway to childhood obesity interventions, in line with systems that currently operate in Dutch primary health care with respect to adult obesity treatments. Although parents may constitute an obstacle in terms of recruitment, YHC professionals could be expected to be optimally equipped to enroll participants for childhood obesity programs. Nevertheless, recruitment problems in obesity prevention interventions have also been reported in the Dutch YHC setting (Veldhuis et al., 2009a). However, no qualitative studies exist that aim to gain insights into the reasons of the recruitment problems in the Netherlands.

The goal of the present study was to identify barriers that Dutch YHC professionals perceive when referring parents of overweight children to an obesity prevention intervention. The study used a qualitative theory-based research design that applied semi-structured interviews.

\section{Methods}

Before the research methodology of the present study is outlined, we will first provide relevant information regarding the childhood obesity intervention and the referral procedure.

\section{Childhood obesity intervention}

The present study is part of the pilot phase of a randomized controlled trial, in which the effectiveness will be tested of a 14-week parent-focused group intervention program. The aim of the intervention is to improve parenting skills and parenting practices related to child's nutrition and physical activity behaviors. The pilot intervention was aimed at parents of overweight children aged 4 years.

\section{Referral procedure}

The referral procedure for the pilot implementation of the obesity prevention intervention consisted of five phases, which are depicted in Figure 6.1. Phase 1 and 2 are part of the current standard procedures of YHC in the Netherlands (Bulk-Bunschoten et al., 2004). At age 3 years and 9 months, children were systematically invited for a preventive visit to child health clinics, where their growth and health behaviors were assessed by a YHC physician. If a child was labelled 'overweight, not obese', according to the sex-and age-specific cut-off points for overweight and obesity based on Cole, Bellizzi, Flegal, \& Dietz et al. 2000 (phase 1), and according to the physicians' clinical 
judgment (based on their experience, expertise and the course of the weight pattern over time) (phase 2), YHC physicians were asked to refer parents to an intervention programme aimed at the prevention of excessive weight gain in 4-year-old overweight children (phases 3 and 4). Parents were approached for participation in the intervention by a member of our study team: parents made their own decision to participate or not (phase 5). In the current study, we tried to identify factors impeding successful implementation of the referral strategy used by the YHC professionals (phases 1-4).

Phase 1: Health professional measures weight and height and calculates BMI

Phase 2: Health professional diagnosis overweight using their clinical judgement

Phase 3: Health professional tries to refer to obesity prevention intervention

Phase 4: Health professional successfully refers to obesity prevention intervention

Phase 5: Parents decide

to participate

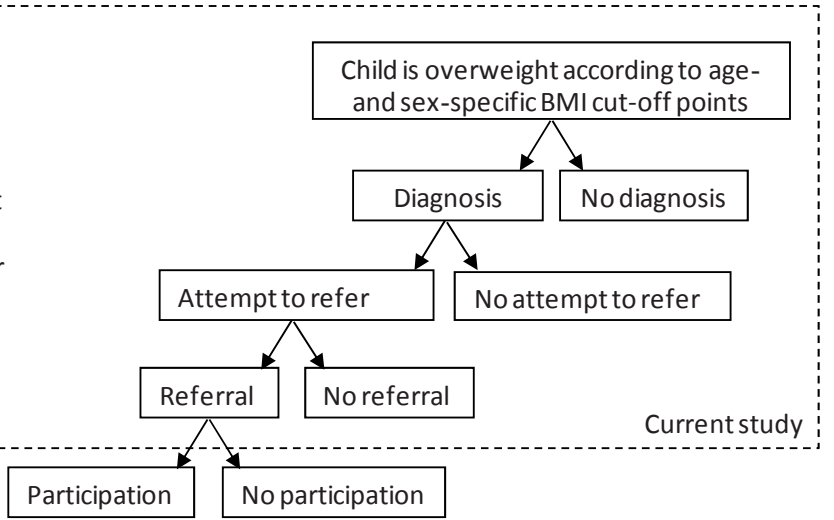

Figure 6.1: Recruitment procedure

\section{Implementation of referral procedure}

As part of the implementation of the referral procedure, a dissemination strategy was developed in order to optimally communicate the procedure with the YHC professionals. In a plenary information session, YHC professionals received information on the intervention and what was expected from them. They also received a written protocol with the guidelines. To ensure that they would not forget to refer eligible parents of overweight children to the intervention, reminders were sent in the form of monthly emails and newsletters. The health professionals were also given an email address and telephone number of the research team, whom they could consult if they had any questions about the procedure. This recruitment procedure was implemented for approximately six months in 14 child health clinics run by two different health organizations in the southern part of the Netherlands (South Limburg). Twenty-five YHC physicians were asked to refer children. Based on YHC records and national overweight prevalence rate in the age group (14\%), an approximate amount of 230 children were eligible for participation in the geographical area during the recruitment period. However, at the end of the recruitment period, the number of referrals proved to be ap- 
proximately $10 \%$ of the eligible children, while we expected this referral rate to be at least double this percentage (Veldhuis et al., 2009a).

\section{Semi-structured interviews}

Semi-structured interviews were conducted as part of an embedded mixed method design (quantitative results are part of an ongoing RCT). The interviews were held with a fairly open framework which allowed focused, conversational, two-way communication. After the six-month implementation period, we asked YHC physicians from the participating child health clinics to participate in semi-structured interviews to identify the reasons for the disappointing number of referrals. To gain a broader insight into potential barriers of recruitment we also invited YHC nurses and management staff members. YHC professionals could register for the interviews when they were willing to participate. All YHC professionals working in the 14 child health clinics were eligible to participate in the interviews. Sixteen YHC professionals participated (response rate $22 \%)$. The interviews were held at the offices of the health professionals in March and April 2010, by the first author of this manuscript, S.G. The current study was part of a larger project which was approved by the ethical committee of the Maastricht University Medical Centre (trial number 10-3-052). All of the participants gave permission for the interviews to be recorded on audiotape. The interviews lasted an average of 20 minutes. All questions were open-ended and concerned a range of topics, including prevention of childhood obesity, appraisal of the intervention and barriers and facilitators to the recruitment of children via YHC. Some example questions are: 'How did you try to refer overweight children to the intervention?', 'What were your experiences in referring children?' 'Did you experience any barriers or stimulating factors in referring children?'.

\section{Research model}

The research model for the study was based on an implementation theory developed by Fleuren, Wiefferink, \& Paulussen (2004). This theory distinguishes five categories of factors which determine the implementation rate of innovations (see Figure 6.2): (1) characteristics of the socio-political environment or the context (e.g., norms and values in society); (2) characteristics of the implementing organization, in this case the YHC organization (e.g., tasks, training and cooperation); (3) characteristics of the implementers, i.e. the YHC professionals (e.g., attitude, self-efficacy, skills, remembering to refer); (4) characteristics of the innovation (e.g., relative advantage, observability (degree to which the results of an innovation are visible (Rogers, 2003)), relevance to the client and frequency of the innovation) and (5) characteristics of the participants, i.e. the parents (e.g., awareness, perceived severity of the problem, resistance, motiva- 
tion, perceived responsibility, willingness to cooperate and parental discomfort about the intervention). The research model was used as a framework for conducting, analyzing and interpreting the interviews.

Characteristics of the socio-
political environment

Characteristics of the YHC
organization

\begin{tabular}{l}
\hline $\begin{array}{l}\text { Characteristics of the YHC } \\
\text { professionals }\end{array}$ \\
\hline
\end{tabular}
Successful implementation of

Characteristics of the innovation

\section{Characteristics of the parents}

Figure 6.2: Research model, based on Fleuren et al. (2004)

\section{Data analysis}

After the interviews had been transcribed, they were coded by two independent reviewers using NVivo 2.0 software. Both reviewers used a coding list which had been drafted before the coding procedure. The framework for analysis of the transcripts was related to the topics in our research model (Figure 6.2). If no suitable code was available, the reviewers together determined the relevance of adding an extra code. After they had both coded the interviews, the reviewers compared their codings, and disagreements were solved in a consensus meeting with a third reviewer.

\section{Results}

\section{YHC professionals}

Interviews were conducted with two management staff members, eight physicians and six nurses. Demographic characteristics of the health professionals are depicted in Table 6.1. All respondents, except one, were female, and their average age was 46 (range 28-61) years. The respondents worked in eleven different child health clinics, almost all were part-timers, and they had an average of 16 years of work experience (range 3-28 years). 


\section{Characteristics of the socio-political context}

With regard to the socio-political context, various societal norms and values were mentioned as hampering the recruitment procedure. Two respondents indicated that norms are changing, so that overweight children are more and more regarded as having a normal weight (see Table 6.2 for citations). Another important view in society is the idea that participation in child overweight programmes is still unusual. One professional also mentioned that people are not aware of the severity of overweight as a problem.

Table 6.1: Characteristics of the YHC professionals

\begin{tabular}{|c|c|}
\hline Practice & $(N=11)$ \\
\hline \multicolumn{2}{|c|}{ Socio-economic status population: } \\
\hline Low & 3 \\
\hline Moderate & 6 \\
\hline High & 2 \\
\hline Participants & $(N=16)$ \\
\hline \multicolumn{2}{|l|}{ Professional Group: } \\
\hline Management & 2 \\
\hline Physician & 8 \\
\hline Nurse & 6 \\
\hline \multicolumn{2}{|l|}{ Gender: } \\
\hline Female & 15 \\
\hline Male & 1 \\
\hline \multicolumn{2}{|l|}{ Age: } \\
\hline$<39$ years & 5 \\
\hline $40-49$ years & 4 \\
\hline$>50$ years & 7 \\
\hline \multicolumn{2}{|l|}{ Years since qualification: } \\
\hline$<10$ years & 4 \\
\hline $10-19$ years & 7 \\
\hline $20-39$ years & 5 \\
\hline \multicolumn{2}{|l|}{ Appointment: } \\
\hline Full-time & 1 \\
\hline Part-time $(<0.5 \mathrm{fte})$ & 10 \\
\hline Part-time ( $\geq 0.5 \mathrm{fte})$ & 5 \\
\hline
\end{tabular}


BARRIERS TO SUCCESSFUL RECRUITMENT I 135

Table 6.2: Stimulating and impeding factors in the recruitment of overweight children

\begin{tabular}{ll}
\hline Factors & Quotations to illustrate the identified factors \\
\hline Socio-political context & \\
$\begin{array}{l}\text { Norms and values in society } \\
\text { Weight-related }\end{array}$ & $(-)$ 'The norm about what is normal weight is changing. Children \\
& who have a healthy weight are now regarded as too lean' \\
& $(-)$ 'Parents don't recognize their child being overweight, because \\
& there's an increase in the number of fat children' \\
& $(-)$ 'It should be regarded as normal that parents participate in such \\
Participation in programmes & a programme' \\
& $(-)$ 'Overweight should get more attention in the media to make \\
Severity of overweight problem & people aware that it's an important health problem'
\end{tabular}

\section{YHC organization}

Task of YHC

Training

Time

Resources

Cooperation

Within the organization

Between organizations
(+) 'Yes, absolutely! I think that no one else will call the parents to account for their child's overweight'

$(+)$ 'Youth health care is the only place where you can find all those young children. There's no other place to reach all children'

(+) 'We are partly responsible, but we're not the only ones who are responsible. Primary schools have responsibilities as well, as they see what the children eat'

(-) 'Extra skills training for professionals on the prevention of childhood overweight is needed in $\mathrm{YHC}^{\prime}$

(-) 'Of course you make time for it, but there's not much time to discuss it, and a lot of other things have to be discussed as well during a consultation'

(-) 'I think we don't have enough resources'

$(-)$ 'Nurses should be more closely involved in the recruitment of overweight children'

(-) 'Unfortunately, we nurses didn't receive any information'

$(-)$ 'We think that we should first try to get all relevant stakeholders to agree'

$(+)$ 'The more consistent people from different organizations are in their message to parents, the higher the chance that we reach them'

(+) 'The municipal health service is also involved in prevention of childhood obesity'

(+) 'It would be better if more organizations were involved. I mean general practitioners, schools, day-care centres'

The YHC professional

Attitude

Programme - specific outcome beliefs $(+)$ 'I think it's a very positive programme, because overweight is mainly an educational problem'

(+) 'Yes, I think it's a nice programme, although it's intensive. It takes a lot of time for the parents' 
136 CHAPTER 6

\begin{tabular}{|c|c|}
\hline Factors & Quotations to illustrate the identified factors \\
\hline \multirow{12}{*}{$\begin{array}{l}\text { Target group - specific outcome } \\
\text { beliefs }\end{array}$} & (-) 'I don’t know. I wonder whether parents see the need for it' \\
\hline & 'I think a particular group does, but I don't think that all parents see \\
\hline & the relevance' \\
\hline & $(+)$ 'We do notice an increased need for parenting support' \\
\hline & $(-)$ 'I think we all thought: how can we motivate these parents? \\
\hline & from the very beginning' \\
\hline & $(-)$ 'A lot of people feel uncomfortable when they hear their child is \\
\hline & overweight. And that makes it hard for us' \\
\hline & $(-)$ 'They're not interested. They have no time for it' \\
\hline & (-) 'I think that one out of thirty people consciously want to change \\
\hline & something' \\
\hline & (-) 'You notice that parents are very unresponsive' \\
\hline \multirow[t]{2}{*}{ Perceived responsibility } & (+) 'I feel responsible and want to discuss it with parents' \\
\hline & $(+)$ 'It's a growing problem' \\
\hline \multirow[t]{8}{*}{ Perceived severity of problem } & $(-)$ 'Obesity is just one of the areas of special interest. I can't say it's \\
\hline & more important than other areas; it's just one of them, although I \\
\hline & take it very seriously!' \\
\hline & $(-)$ 'To be honest, I think that a lot of children between S1 and S2 \\
\hline & are actually not too fat' \\
\hline & $(-)$ 'My experience is that the majority of the overweight children \\
\hline & are just above the norm. When you look at that child, using your \\
\hline & clinical judgement, I think those children are not overweight' \\
\hline \multirow[t]{4}{*}{ Need for prevention } & (+) 'Prevention of childhood obesity is really important, especially \\
\hline & to prevent long-term risks' \\
\hline & $(-)$ 'I think that prevention of psychosocial problems is more \\
\hline & important, but by that I mean severe problems like neglect' \\
\hline \multirow[t]{3}{*}{ Self - efficacy } & (-) 'I think it’s a complicated problem' \\
\hline & $(-)$ 'Sometimes parents were very critical and started asking me a \\
\hline & lot of questions, which I couldn't answer. I felt uncomfortable' \\
\hline \multirow[t]{4}{*}{ Skills } & $(-)$ 'We're not able to communicate the impact of the problem to \\
\hline & the parents. We need more practice in communication skills' \\
\hline & (-) 'I think we don't have enough expertise about prevention of \\
\hline & childhood obesity' \\
\hline Forgetting & (-) ‘I have to admit, I had forgotten it after a while' \\
\hline
\end{tabular}

The innovation

Relative advantage

'I think that the current protocol and the intervention can complement each other'

$(+)$ 'An advantage of the intervention is that professionals who are experienced in childhood obesity give the parents advice'

(-) 'I think that we're already quite effective in our own approach'

$(+)$ 'I think it's a very positive programme, because overweight is mainly an educational problem'

Observability

(-) ‘ I would prefer to get more timely feedback on which parents participated and which ones didn't' 


\begin{tabular}{|c|c|}
\hline Factors & Quotations to illustrate the identified factors \\
\hline Relevance for the client & $\begin{array}{l}(+) \text { 'I think that the programme is very useful for parents. Childhood } \\
\text { overweight is a problem which is closely related to parenting' }\end{array}$ \\
\hline Low frequency of use innovation & (-) 'No, I didn't see any children who were eligible for participation' \\
\hline \multicolumn{2}{|l|}{ The parents } \\
\hline $\begin{array}{l}\text { Awareness of child's } \\
\text { overweight }\end{array}$ & $(-)$ 'Parents are not aware of their child's overweight' \\
\hline \multirow{4}{*}{$\begin{array}{l}\text { Perceived severity of child's overweight } \\
\text { Resistance in discussing weight issues } \\
\text { with parents }\end{array}$} & (-) 'Parents often don't see their child's overweight as a problem' \\
\hline & (-) 'You clearly notice that parents are unresponsive' \\
\hline & $\begin{array}{l}\text { (-) 'You notice that when you mention the word 'overweight' to } \\
\text { parents, you immediately perceive resistance' }\end{array}$ \\
\hline & $\begin{array}{l}(-) \text { 'Sometimes parents become angry when you continue to } \\
\text { discuss overweight' }\end{array}$ \\
\hline Motivation to change & $(-)$ 'Parents are just not motivated to change their behavior' \\
\hline behavior & $\begin{array}{l}\text { (-) 'It's difficult to approach parents for overweight prevention; } \\
\text { they just don't see the long-term advantages' }\end{array}$ \\
\hline Perceived responsibility & $\begin{array}{l}(-) \text { 'Parents don't admit that they are themselves responsible for } \\
\text { the weight of their child' }\end{array}$ \\
\hline \multirow[t]{2}{*}{$\begin{array}{l}\text { Willingness to cooperate in the } \\
\text { intervention }\end{array}$} & $\begin{array}{l}(-) \text { 'Some parents want to reduce their child's overweight } \\
\text { themselves' }\end{array}$ \\
\hline & $\begin{array}{l}(-) \text { 'They mention that they are closely watching their child's weight } \\
\text { themselves and that they already know what to do about it' } \\
(-) \text { 'A lot of parents don't have time for it. Or they don't want to } \\
\text { make time for it' }\end{array}$ \\
\hline $\begin{array}{l}\text { Parent's discomfort about } \\
\text { the intervention }\end{array}$ & $\begin{array}{l}(-) \text { 'Parents think that it's going too far to participate in an } \\
\text { intervention' }\end{array}$ \\
\hline
\end{tabular}

Note: (+) stimulating factors, (-) impeding factors

\section{Characteristics of the YHC organization}

All respondents considered prevention of childhood obesity to be an important task of the YHC organization, although some professionals commented that other stakeholders had responsibilities as well. General practitioners, schools, day-care centres and municipal health services were mentioned as other potentially relevant stakeholders.

Few YHC professionals reported a need for extra skills training on the management of childhood obesity. Some of them indicated that they had insufficient time during the consultations to refer children to the intervention, or that not enough resources were available. As regards cooperation, some interviewees indicated that it would be important to involve nurses in the recruitment of children as well, because they often know more about the children's background. 


\section{Characteristics of the YHC professionals}

A number of socio-cognitive factors of the YHC professionals were identified as potentially stimulating or impeding factors in the recruitment of overweight children. Respondents mentioned both outcome beliefs about the programme as well as beliefs about the target group as influencing the recruitment of overweight children. The majority of the respondents were positive about the intervention; they thought the intervention was useful, although some physicians mentioned that it was intensive and time-consuming. In general, respondents were less positive about whether they expected the target group to cooperate in the innovation. When professionals expect that the target group does not want an intervention or does not see the need for it, they may be less likely to refer. Most of the interviewees perceived the prevention of childhood obesity to be among their responsibilities. The interviewees reported mixed beliefs regarding the perceived severity of the problem. Although more than half of the respondents perceived childhood overweight and obesity as a serious problem, some mentioned that children who were overweight according to the guidelines were not always overweight according to their clinical judgement. All respondents considered prevention of overweight to be important.

A few respondents indicated that their skills (e.g. communication skills) in the prevention of childhood obesity could be improved, and some professionals mentioned that they had low self-efficacy. Both a lack of communication skills and low selfefficacy were expected to hamper the recruitment of parents of overweight children. A final barrier was that some professionals forgot to implement the recruitment procedure.

\section{Characteristics of the innovation}

Various characteristics of the intervention were mentioned by the interviewees as influencing the recruitment of parents of overweight children. Respondents' views about the relative advantage of the intervention were mixed. Although some mentioned clear advantages, others thought another new intervention to manage childhood obesity was superfluous. Regarding the observability of the recruitment procedure, some respondents mentioned that it was not always clear to them whether the parents they referred actually participated in the intervention. Nevertheless, almost all respondents were convinced of the relevance of an obesity prevention programme for parents. One third of the respondents indicated that they had had no eligible participants in their clinic during the recruitment period, and they had therefore not been parents able to refer of overweight children. 


\section{Characteristics of the parents}

The majority of the professionals indicated that parents seemed to be unaware of their children's overweight, or unaware of the health consequences (low perceived severity). Several respondents indicated that they had encountered resistance on the part of the parents when they tried to discuss their children's weight or overweight with them. Some respondents had even found that parents became angry. Almost all YHC professionals also observed a lack of motivation among parents to change behavior, sometimes because people did not recognize the advantages of behavioral change. Some respondents mentioned that some parents had low perceived responsibility; they appeared not to understand that they were partly responsible for their child's weight. Others indicated that some parents said they preferred to try and reduce their child's weight themselves. Some parents had also said that they did not have enough time, or had argued that they did not see the need to participate in an intervention.

\section{Discussion}

The current study investigated the views of Dutch youth health care (YHC) professionals regarding barriers to referring parents of overweight children to an obesity prevention intervention. Although the Dutch YHC system appears an excellent opportunity to identify and refer overweight children, and all interviewed YHC professionals were concerned about childhood obesity and perceived prevention of overweight and obesity as an important task of the YHC organization, a range of barriers impeded the optimal referral. In terms of frequency and perceived impact, the most important aspects hampering the referral procedure were reported to be factors relating to the parents of the overweight children. In particular, denial of the overweight problem and resistance towards discussing weight issues were often mentioned by respondents as impeding factors. Some interviewees reported themselves to be unable to motivate parents to participate in an obesity intervention. Their (self-perceived) insufficient skills and low self-efficacy towards motivating parents are likely to hamper recruitment. Other relevant factors hindering the recruitment process were related to the organization (e.g., lack of time, lack of resources, lack of skills training), societal norms related to weight status, and societal norms towards participation in child overweight programs. In fact, the changing norm in society towards 'normal weight' may also have been visible in the observation that some of the children that were referred were actually obese. The acceptance of early interventions in society is expected to rise when evidence-based interventions become more prevalent and easily accessible. This may involve that obesity prevention interventions are not presented as part of the primary health care domain, but as part of the public health domain. Recruitment through 
public settings such as schools and communities may then complement YHC referral strategies.

No previous study has identified barriers that Dutch YHC professionals perceive when referring parents of overweight children to an obesity prevention intervention. Earlier studies in other health care contexts did however also report barriers to managing childhood obesity as perceived by physicians or nurses. In parallel to our results, those studies also identified perceived difficulties among YHC professionals in discussing weight issues with parents (Moyers, Bugle, \& Jackson, 2005; Rice et al., 2008). Barriers were reported especially when physicians experienced personal weight challenges (Steele et al., 2011), or in situations when children lacked motivation (Spivack, Swietlik, Alessandrini, \& Faith, 2010; Story et al., 2002; Veldhuis et al., 2009a), and when there was a lack of family involvement or motivation (Spivack et al., 2010; Steele et al., 2011; Story et al., 2002; Veldhuis et al., 2009a), a lack of support services (Kubik, Story, \& Davey, 2007; Steele et al., 2011; Story et al., 2002) or a lack of time (Jelalian, Boergers, Alday, \& Frank, 2003; Kubik et al., 2007; Steele et al., 2011; Turner, Shield, \& Salisbury, 2009; Walker, Strong, Atchinson, Saunders, \& Abbott, 2007). Our study showed that in general, YHC professionals do acknowledge the relevance of early prevention. Some of them indeed did not see the advantage of our parent-focused group intervention program, which may also be a reason for a lack of referrals. YHC professionals do acknowledge their professional responsibility to raise the issue of excessive weight gain in children. However, they appear uncomfortable and unequipped to do this. Previous research among physicians has also found that low perceived skills (Steele et al., 2011; Story et al., 2002; Turner et al., 2009), low self-efficacy (Miller Perrin, Flower, Garrett, \& Ammerman, 2005; Moyers et al., 2005; Steele et al., 2011; Story et al., 2002), and low priority for the overweight problem (Senden \& Krumeich, 2011), hamper the recruitment of overweight children for prevention and treatment programmes.

Poor detection of overweight (Finne et al., 2009), probably due to low use of BMIfor-age (Redsell et al., 2011; Wethington, Sherry, \& Polhamus, 2011), has also been indicated to be a problem in referral strategies. Indeed, it is questionable whether an obesity prevention intervention should be advocated for a child identified as just in the overweight range based on a single assessment. It would be even more challenging to raise the topic of overweight with parents of children that have always been in the healthy weight range and are just in the overweight range at one point in time. Dutch guidelines, however, do not incorporate only one measurement in the classification of overweight in children. YHC physicians take the course of children's weight development over time into account in determining whether a child is overweight (i.e. 'clinical judgment'). This clinical judgement is a vague criterion which may make it relatively difficult to refer parents. This could be an interesting topic for future clinical studies, 
also in order to provide feedback to YHC professionals with the goal to optimize their clinical judgement skills. Some parents, however, appear not to consider their child to be overweight, even though the age- and sex-specific BMI cut-off points indicate they are (De La, 2009; Oude Luttikhuis et al., 2010; Towns \& D'Auria, 2009). And parents who do recognize that their child is overweight sometimes lack a perceived need to manage their child's weight (Finne et al., 2009; Veldhuis et al., 2009a).

Some strengths and limitations of the present study should be acknowledged. Strengths include the theoretical basis and the use of a qualitative research design, which adds to the richness of the data. We used a research framework that was based on implementation theory to guide the interview structure. The semi-structured qualitative interviews enriched the contents of the broad concepts that were included in the applied research framework. Thus, we ensured that all potentially relevant concepts were addressed in the interviews and we succeeded in getting a grip on the most important beliefs of the interviewed YHC professionals within each concept. All interviews were conducted by the same researcher in order to increase consistency in that data gathering process, and were coded by two independent researchers to increase confirmability (objectivity and neutrality). Limitations of the current study include the selectivity of the sample and the risk of socially desirable answers that is inherent in interviews. Since participation in the interviews was voluntary this may limit the generalisability of the study. We did not have the impression that social desirability was a problem. The professionals appeared to be open in stating their opinions and by admitting their shortcomings and lack of skills. Also, the atmosphere during the interviews was quite confidential. Another limitation of the current study is that we focused exclusively on YHC professionals. YHC professionals could have displayed an external locus of control when they mention that the parents are the main reason for not referring children. On the other hand, some professionals admitted that they have too few skills to communicate effectively with the parents, thereby internally attributing the low referral rates. Studies that focus on the parent perceptions may provide more information on this.

Based on the present results, we consider it important that YHC physicians and nurses receive more training in interview techniques to strengthen their self-efficacy towards discussing weight issues with parents. Motivational Interviewing (MI) may be a fruitful communication strategy in this respect. MI is a client-oriented method based on the use of communication skills to understand individuals' motivation for change (Miller \& Rollnick, 2002). Studies have shown that MI can be a valuable health behavior change intervention for children and their parents (Suarez \& Mullins, 2008), which can also be used in the treatment of childhood obesity (Schwartz et al., 2007; Taveras et al., 2011). The technique has been successfully applied in health care settings (Lozano et al., 2010). To increase its effectiveness, the technique should not just be an 
important intervention component, but should also become a permanent part of professionals' routine work patterns. Furthermore, a better understanding of parental resistance and denial in relation to child overweight could be gained by future research investigating parents' views about discussing weight-related issues with health professionals.

\section{Conclusions}

The YHC context and the professionals' attitude towards prevention of childhood obesity do not appear to be major barriers for referring parents of overweight children to an obesity prevention intervention. By contrast, the professionals particularly perceived factors relating to the parents of the overweight children as impeding optimal referral. This lack of parental awareness towards their child's overweight should be addressed in future studies. In addition, YHC professionals' communication skills and self-efficacy in discussing weight issues with parents of overweight children appear insufficient. In addition to efforts to optimize the efficacy of obesity prevention interventions, it will be fruitful to emphasize the importance of increasing the reach of such programmes, for example by training YHC professionals in motivating parents of overweight children to participate in obesity prevention interventions. 


\section{CHAPTER 7}

\section{Lifestyle Triple P:}

a parenting intervention for childhood obesity study protocol

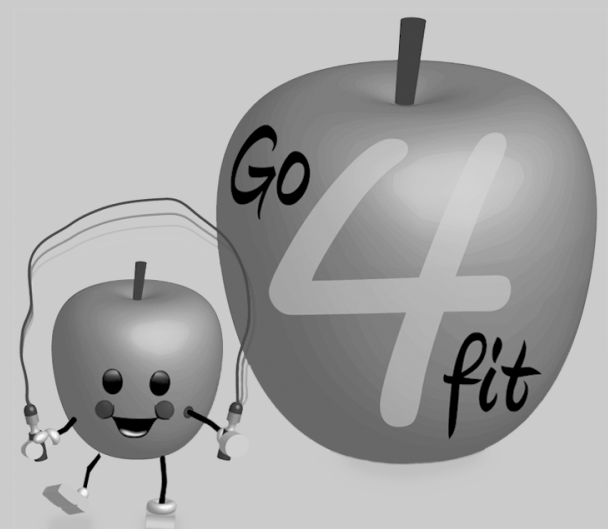

Published as: Gerards, S.M.P.L., Dagnelie, P.C., Jansen, M.W.J., Van der Goot, L.H.M., De Vries, N.K., Sanders, M.R., Kremers, S.P.J. (2012). Lifestyle Triple P: a parenting intervention for childhood obesity. BMC Public Health, 12: 267 


\section{Abstract}

Background: Reversing the obesity epidemic requires the development and evaluation of childhood obesity intervention programs. Lifestyle Triple P is a parentfocused group program that addresses three topics: nutrition, physical activity, and positive parenting. Australian research has established the efficacy of Lifestyle Triple $P$, which aims to prevent excessive weight gain in overweight and obese children. The aim of the current randomized controlled trial is to assess the effectiveness of the Lifestyle Triple $P$ intervention when applied to Dutch parents of overweight and obese children aged 4-8 years. This effectiveness study is called GO4fit.

Methods/Design: Parents of overweight and obese children are being randomized to either the intervention or the control group. Those assigned to the intervention condition receive the 14-week Lifestyle Triple $P$ intervention, in which they learn a range of nutritional, physical activity and positive parenting strategies. Parents in the control group receive two brochures, web-based tailored advice, and suggestions for exercises to increase active playing at home. Measurements are taken at baseline, directly after the intervention, and at one year follow-up. Primary outcome measure is the children's body composition, operationalized as BMI z-score, waist circumference, and fat mass (biceps and triceps skinfolds). Secondary outcome measures are children's dietary behavior and physical activity level, parenting practices, parental feeding style, parenting style, parental self-efficacy, and body composition of family members (parents and siblings).

Discussion: Our intervention is characterized by a focus on changing general parenting styles, in addition to focusing on changing specific parenting practices, as obesity interventions typically do. Strengths of the current study are the randomized design, the long-term follow-up, and the broad range of both self-reported and objectively measured outcomes.

Trial Registration: Current Controlled Trials NTR 2555. MEC AzM/UM: NL 31988.068.10/ MEC 10-3-05 


\section{Background}

Overweight and obesity are having increasing public health impact worldwide. In 2003, between $12 \%$ and $19 \%$ of 4 - to 8 -year-old children in the Netherlands were overweight, and about 2 to $4 \%$ were obese (Van den Hurk, Van Dommelen, Van Buuren, Verkerk, \& Hirasing, 2007). More recent data (2007 - 2010) show that $17 \%$ of the 7 - to 8 -year-old children are overweight and $5 \%$ are obese (Van Rossum, Fransen, VerkaikKloosterman, Buurma-Rethans, \& Ocké, 2011). Overweight children are at increased risk of becoming obese adults (Singh et al., 2008) and of developing cardiovascular diseases or type 2 diabetes (Must \& Strauss, 1999). Moreover, these children often suffer from social consequences like teasing and discrimination (Washington, 2011) which may influence their mental health. It is therefore important to develop and evaluate interventions to reverse this trend of increasing prevalence. In recent decades, childhood obesity interventions have been developed and evaluated, with some promising results (Bluford et al., 2007; Doak et al., 2006; Kitzmann et al., 2010), but there is still a lack of sound theory- and evidence-based interventions to prevent overweight in children (Summerbell et al., 2005).

There are several reasons for focusing efforts to prevent excessive weight gain on children at a relatively young age. First, overweight at a young age predicts excessive weight gain in the future (Magarey et al., 2003). Also, children have a relatively short history of unhealthy habits, which may make it easier to change these behaviors compared to adult populations. Finally, prevention of excessive weight gain in children is based on a different mechanism than prevention of weight gain in adults: children can reduce their BMI while growing without losing weight, whereas adults have to lose weight in order to reduce their BMI (Kremers et al., 2008).

The role of the parents in the development of children's weight status is increasingly emphasized in intervention studies on the treatment and prevention of childhood obesity (Golley et al., 2011; Kitzmann et al., 2010). Parents are the primary caregivers, who are largely responsible for their children's nutrition and physical activity patterns, particularly in the early years of life. It is therefore important to target both general parenting styles and parenting practices. Unlike parenting practices, which focus on specific parenting behaviors relating to aspects like dietary behavior and physical activity, parenting styles refer to parent-child interactions across a wide range of situations. Parenting styles are regarded as the context in which behavior-specific parenting takes place (Darling \& Steinberg, 1993; Sleddens et al., 2011). A recent review indicated that interventions aimed at changing parenting styles are effective in the prevention and management of childhood obesity (Gerards et al., 2011). In addition, parenting interventions seem to have positive long-term effects on a range of other youth outcomes, 
viz. mental, emotional and behavioral disorders, as well as on successful developmental competence (Sandler, Schoenfelder, Wolchik, \& Mackinnon, 2011).

The Triple P Positive Parenting Program is a multi-level parenting and family support strategy developed by the University of Queensland in Brisbane, Australia (Sanders et al., 2003). Triple P is based on social learning principles and adopts a system-contextual or ecological perspective in supporting parents. The program is internationally supported and several derivative programs have been developed to address parents' special needs. One of these derivative programs is Lifestyle Triple P (West \& Sanders, 2010). It is a parent-focused group program that addresses three topics: nutrition, physical activity, and positive parenting. The efficacy of Lifestyle Triple $\mathrm{P}$ has been tested in a randomized controlled trial (RCT) in Australia (West et al., 2010b). West and colleagues showed that their intervention significantly decreased children's body size and body fat, decreased their weight-related problem behavior, increased parental confidence in managing weight-related problem behavior, and decreased ineffective parenting (West, 2007; West et al., 2010b). The intervention has not been tested outside Australia. In addition, a limitation noted by the authors of the Australian study was that their measures of lifestyle patterns had low reliability and sensitivity (West, 2007), and that they had not measured the effects of the intervention on other family members (parents or siblings). Based on the process evaluation of the Australian study, in which parents indicated that the program was too short, the original 12 session Lifestyle Triple P program was extended to 14 sessions. Before implementation of the intervention on a national scale in the Netherlands, we decided to assess the effects of the adapted version of Lifestyle Triple $P$ in the Dutch context.

The aim of the current RCT is to assess the effectiveness of the Lifestyle Triple P intervention in the Netherlands in addressing overweight and obese children aged 4-8 years. The study has been named GO4fit, and aims to assess changes in children's anthropometric outcomes, anthropometric outcomes of other family members (parents and siblings), parenting practices, parental feeding style, parenting style, parental self-efficacy and children's energy balance-related behaviors.

\section{Methods/design}

\section{Study design}

The effectiveness of the Lifestyle Triple $P$ intervention is being tested in the southern part of the Dutch province of Limburg, using an RCT design. After baseline measurements, parents of overweight children are randomly allocated to either the intervention group or the control group. The intervention group receives the Lifestyle Triple $\mathrm{P}$ intervention, whereas parents in the control group receive information on healthy 
nutrition, physical activity, and positive parenting. To evaluate the effect of the intervention, participants are measured again immediately following the intervention (4 months after baseline), and at 12 months after baseline (see Figure 7.1 for the study design). Baseline measurements and intervention groups are started as soon as enough participants per location have been recruited (i.e. a minimum of 10: 5 for the control group and 5 for the intervention group). The Medical Ethics Committee of the University Hospital Maastricht and Maastricht University approved the study protocol (reference number NL 31988.068.10 / MEC 10-3-052).

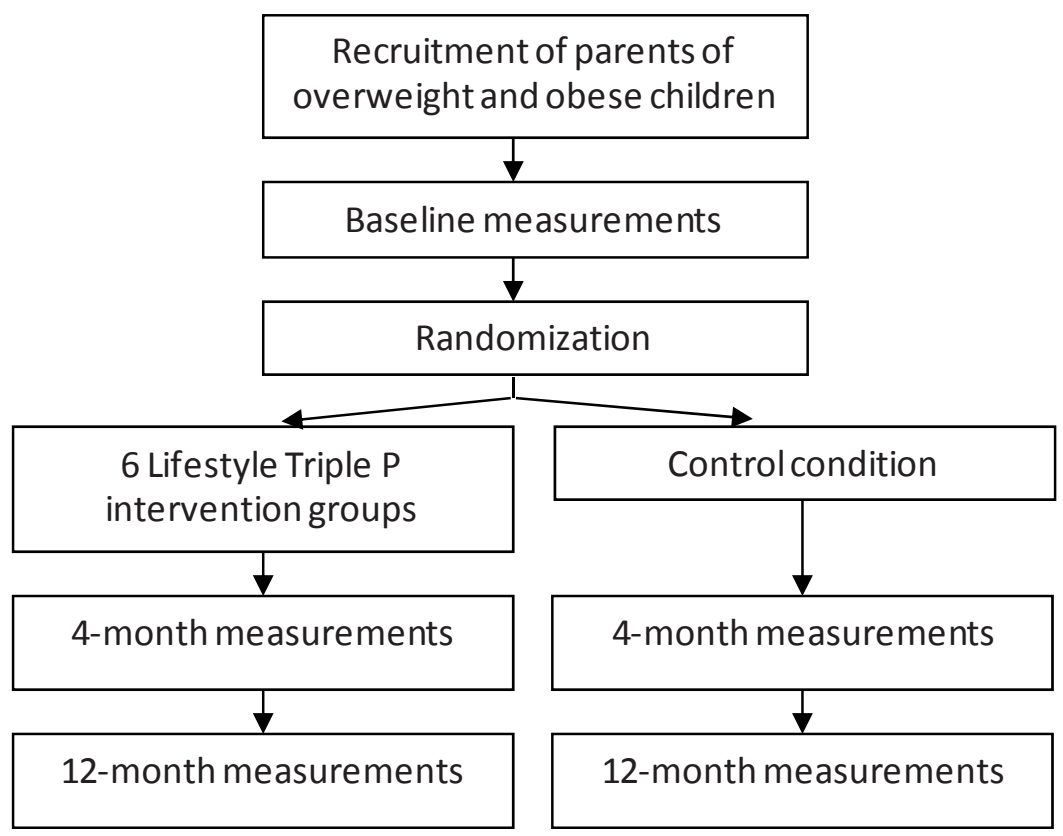

Figure 7.1: Study design

\section{Target population}

The Southern Limburg regions has 608,885 inhabitants (Lamberts et al., 2010), 49.8\% $(303,225)$ of whom live in the municipalities of Maastricht, Sittard-Geleen and Heerlen. About $10 \%$ are aged 4 to 8 years. The estimated prevalences of overweight and obesity in the $4-8$ year age group are $9.5 \%$ and $2.3 \%$ respectively, implying an eligible population of 3,578 children for the current study. 


\section{Recruitment of the study sample}

The participants are being recruited using four strategies: two strategies that use the Dutch Youth Health Care (YHC) system, one using mass media and one using personal letters. Health professionals working in the YHC system have been instructed to refer parents of overweight children to the Lifestyle Triple $\mathrm{P}$ intervention. In addition, parents of children who are overweight according to the YHC's medical records have been approached for participation in the intervention. Finally, mass media materials (a brochure, a poster, advertisements in newspapers, and a website) have been developed to inform parents about the intervention and to offer them the opportunity to register for the intervention. We have also sent invitation letters to parents of primary school children. Recruitment of participants for the current RCT started in December 2010.

\section{Participants}

We aim to recruit a study population of 84 child-parents triads. Parents of children aged between 4 and 8 years (at inclusion) are eligible for participation if their child is considered overweight or obese, based on the $\mathrm{BMI}$, using the international sex- and age-specific cut-off points proposed by Cole et al (2000). Parents who agree to participate in the study and both sign the informed consent form are included in the current study.

\section{Power calculation}

The sample size calculation was based on the difference between the intervention condition and the control condition in terms of BMI points. A difference of $0.30 \mathrm{BMI}$ points between the intervention and control conditions was expected to be relevant, based on previous studies, e.g. Robinson 1999. Based on an alpha value of 0.05 and a power of 0.90 , adjusting for attrition and nesting effects within the groups, we aimed to randomly assign 84 families to the 6 Lifestyle Triple P groups (intervention) or to the control condition.

\section{Randomization}

The randomization scheme has been generated by an independent researcher (PCD), who is not directly involved in the data collection or intervention delivery, using a block size of four and sealed envelopes. The randomization is concealed to all other members of the study team. After baseline measurements, participants are randomly allocated to either the intervention or the control condition. A member of the study team (SMPLG), who is blinded to the randomization scheme, calls the research institute by telephone in the presence of the parents to enquire for their group allocation, and then informs the parents. 


\section{Lifestyle Triple P Intervention}

\section{Objectives}

Key objectives of the Lifestyle Triple P intervention are:

- improving children's dietary intake, activity levels, and weight status;

- increasing parenting skills and confidence in managing children's weight-related behavior;

- $\quad$ reducing parents' use of coercive and permissive discipline practices to their children;

- $\quad$ improving parents' communication about health and nutrition;

- $\quad$ reducing parenting stress associated with raising healthy children.

\section{Structure}

The participants who are assigned to the intervention condition receive a 14-week Lifestyle Triple $\mathrm{P}$ intervention which consists of eight weekly 90 -minute parental group sessions, followed by two weekly 15-30 minute telephone sessions, one further 90minute group session, two weekly 15-30 minute telephone sessions, and a final 90minute group session. The groups are composed of either one or both parents of at most 9 families, and each group is led by two Lifestyle Triple P facilitators. These are health professionals who have been accredited after attending an official 3-day Triple $P$ training course and an additional Lifestyle Triple $P$ training day. The group sessions take place at three different buildings of the regional Public Health Services (at Heerlen, Geleen, and Maastricht). To ensure intervention fidelity (defined as the extent to which the intervention is implemented as intended (Dusenbury, Brannigan, Falco, \& Hansen, 2003)), the facilitators meet on a regular basis with a member of the study team (SMPLG) for supervision. Participation in the intervention is free of charge. Each family is provided with a parent workbook, a recipe book, and an active games booklet to support the information presented in the sessions. All the materials have been translated into Dutch for the current trial, according to the following process. First, translators of Triple $\mathrm{P}$ International translated the materials from English to Dutch. Hereafter, all translations were reviewed by a native Dutch speaker (SMPLG). The translation and reviewing process were conducted in line with already existing Dutch Triple $\mathrm{P}$ materials. A pilot study was undertaken to test specific parts of the Lifestyle Triple P intervention for feasibility and acceptability.

\section{Content}

Lifestyle Triple is a family intervention strategy to prevent and treat childhood obesity. The intervention consists of active skills training methods based on self-regulation 
principles, to provide parents with new knowledge and skills. In the first sessions, parents individually formulate realistic goals for change, with the help of the trainer and based on their child's dietary intake and activity levels. Parents are instructed on a range of nutrition strategies (e.g., establishing eating routines and providing healthy foods), physical activity strategies (e.g., providing active games and playing with your child), and positive parenting strategies (e.g., spending quality time with your child and giving frequent praise). An overview of the intervention topics is shown in Table 7.1. The telephone sessions aim to provide parents with individual support in the implementation of strategies at home.

\section{Control condition}

Participants who are randomized to the control condition receive two brochures (one on healthy nutrition and physical activity, and one on positive parenting), web-based tailored advice on setting a good example to their child, and suggestions for exercises to increase active play at home.

\section{Outcome measures}

Outcome measures are assessed at baseline (one or two weeks before the start of the intervention), at 4 months (immediately after the intervention), and at one year follow-up. Primary outcome measure is children's body composition, operationalized as BMI z-score, waist circumference, and fat mass. Secondary outcome measures are children's dietary behavior and physical activity level, parenting practices, parental feeding style, parenting styles, parental self-efficacy, and body composition of family members (parents and siblings).

\section{Anthropometry}

Anthropometric measurements in the children are conducted during a visit to the Public Health Service office. If one or both parents are willing to participate, they are measured as well. In addition, if siblings (if applicable) are present at the visit and are willing to participate, they are also measured. Measurements are performed by a YHC professional who is blinded for group allocation, using a standardized protocol. The participating YHC professional has many years of experience in performing body measurements and has attended a one-day training course to perform the measurements in accordance with the protocol. 
LIFESTYLE TRIPLE P: STUDY PROTOCOL | 151

Table 7.1: Overview of the topics and strategies in each Lifestyle Triple P session

Session Topics Strategies

Session 1:

Preparing for change

Session 2:

Understanding nutrition

Session 3:

Understanding physical activity

Session 4:

Using rewards and modifying recipes

\section{Session 5:}

Limiting sedentary activity and

reading food labels

Session 6:

Playing active games

\section{Session 7:}

Providing healthy meals

\section{Session 8:}

Managing problem behavior

\section{Session 9:}

Using Lifestyle Triple P strategies 1

Session 10:

Using Lifestyle Triple P strategies 2

Session 11:

Planning ahead

\section{Session 12:}

Using planned activities 1

Session 13:

Using planned activities 2

Session 14:

Program Close
Nature and causes of obesity

Overview of Lifestyle Triple P

Readiness to change

Increasing children's self-esteem

Food groups and daily servings

Nutrition goals

Encouraging healthy behaviors

Physical activity goals

Increasing incidental activity

Reducing sugar intake

Using behavior charts

Reducing fat intake

Modifying recipes

Limiting sedentary activities

Establishing ground rules

Reading food labels

Providing active alternatives

Improving movement skills

Establishing eating routines

Providing healthy meals/snacks

Children's participation in sport

Managing problem behaviors

Implementing strategies

Implementing strategies

Family survival tips

High-risk situations

Planned activities routine

Implementing planned activities

routine

Implementing planned activities

routine

Progress review

Maintaining changes

Problem solving for the future
Spending quality time with your child

Talking to your child

Showing affection

Giving frequent praise

Setting a good example

Replacing foods high in added sugar

Providing water as a regular drink

Spending family leisure time in active ways

Encouraging active means of transport

Using behavior charts

Buying low-fat foods

Using low-fat cooking methods

Replacing high-fat ingredients

Establishing clear ground rules

Using targeted discussion to deal with rule-

breaking

Reading food labels

Limiting sedentary activities

Providing active games

Playing with your child

Establishing eating routines

Providing healthy meals and snacks

Encouraging participation in sport

Using planned ignoring for minor misbehavior Giving clear, calm instructions

Backing up instructions with consequences, quiet time, or time-out 
Weight is measured using an electronic portable scale (standardized Seca 899) to the nearest $0.1 \mathrm{~kg}$, with the subject wearing only underwear. Height is measured using a portable stadiometer (Seca 214) with an accuracy of $1 \mathrm{~mm}$. Both measurements are used to calculate BMI and BMI z-scores. Waist circumference is measured with a flexible tape to the nearest $1 \mathrm{~mm}$. Biceps and triceps skinfold thickness is measured to the nearest $0.1 \mathrm{~mm}$ using a Harpenden skinfold caliper. Each skinfold is measured three times and the median is used. If two of the scores differ by more than $10 \%$, another three skinfolds are measured, and the median of the six values is used.

\section{Accelerometry}

Children's physical activity levels are measured using an Actigraph accelerometer (Actigraph, Pensacola, Florida). Children are asked to wear the accelerometer for seven consecutive days, preferably in the week after the anthropometric measurements. The epoch (time frame) is set at $15 \mathrm{sec}$.

\section{Questionnaire}

Parents are asked to fill out a questionnaire at baseline, at posttest and at one year follow-up. The questionnaire has been compiled from validated scales, and assesses the following variables:

- Demographics: gender, age, household and family composition, educational level of the parents, ethnicity of the child and the parents, and work situation of the parents;

- Energy balance-related behaviors of the child: screen-viewing behavior, snacking behavior, soft-drink consumption, fruit and vegetable consumption and physical activity level (Gubbels et al., 2011a);

- Parenting practices: monitoring, restriction, pressure to eat, and perceived responsibility (Birch et al., 2001);

- Parental feeding style: instrumental feeding, emotional feeding, control, and encouragement (Wardle, Guthrie, Sanderson, \& Rapoport, 2001);

- Parenting style: restrictiveness and nurturance (Dekovic, 1989) and psychological control (Barber, 1996);

- Parenting self-efficacy: satisfaction about one's own efficacy and effectiveness at solving problems (Johnston \& Mash, 1989);

- Personality characteristics of the child: surgency/extraversion, negative affectivity, and effortful control (Rothbart, Ahadi, Hershey, \& Fisher, 2001).

- Parental satisfaction with the intervention is measured by including process evaluation questions in the post-test questionnaire at 4 months, and questions about 
changes in problem behavior and management of problem behavior in the 1-year questionnaire.

\section{Statistical analysis}

Descriptive statistics will be used to describe subject characteristics, including baseline values of primary and secondary outcome measures. Continuous variables will be presented as means and standard deviations. Categorical data will be presented as percentages of respondents within each of the possible categories.

Both univariate and multivariate multilevel analyses (to control for potential nesting effects within the Lifestyle Triple $\mathrm{P}$ groups) will be conducted to determine the effect of the intervention on changes in children's BMI z-score, children's waist circumference, children's fat mass, children's lifestyle (screen-viewing behavior, snacking behavior, soft-drink consumption, fruit and vegetable consumption, and physical activity level), parenting self-efficacy, parental skills, and the BMI, waist circumference, and fat mass of both the parents and the siblings. All analyses will be performed according to intention-to-treat analyses, and additional per protocol analyses will be performed. Models will be adjusted for relevant confounders such as children's age, gender, and ethnicity, and parental socio-economic status.

\section{Discussion}

The purpose of this paper was to describe the design of the GO4fit study, which is currently testing the effectiveness of the Lifestyle Triple $P$ intervention in the Netherlands.

Strengths of the current study include its RCT design, the long-term follow-up, and the broad range of both self-reported and objectively assessed outcome measures. Challenges of the study are mainly related to recruitment issues and intervention implementation. Recruitment issues are due to the difficulty of fitting the intervention into the Dutch Youth Health Care system (Gerards et al., 2012a), parents' underestimation of their child's weight status (Oude Luttikhuis et al., 2010), and low recognition among parents of the relevance of the intervention. Intervention implementation issues are related to high quality intervention delivery and intervention fidelity.

In an effort to involve important stakeholders in the recruitment of participants and the implementation of the Lifestyle Triple $P$ intervention, we have secured the support of the Academic Collaborative Centre for Public Health in Limburg. The Academic Collaborative Centre represents a collaboration between policy (municipal au- 
thorities), practice (Public Health Service) and research (Maastricht University) with the aim of improving public health (Jansen, De Vries, Kok, \& Van Oers, 2008). We have tried to further optimize the implementation of the Lifestyle Triple $P$ intervention in the Netherlands (e.g., translation of the intervention materials, training of the Lifestyle Triple $P$ facilitators) by collaborating with the Family Support Centre of the University of Queensland, Brisbane, Australia.

If the intervention proves effective, implementation studies will be needed, including research on ways of reaching low-SES groups and ways of integrating the program in existing YHC structures. A notable characteristic of the intervention is its focus on changing general parenting styles, in addition to solely focusing on changing specific parenting practices, as obesity interventions typically do (Kitzmann \& Beech, 2006). We expect that interventions focusing on changing general parenting styles will have a large and sustained impact on the children's energy balance-related behavior. In addition, we expect these interventions to have an impact on a broad range of specific other parenting practices relating to multiple child outcomes (Kitzmann \& Beech, 2006), indicating a potentially large public health impact (Gerards et al., 2011). The first results of the RCT are expected end 2013. 


\section{CHAPTER 8}

\section{The effectiveness of Lifestyle Triple $P$ in the} Netherlands:

a randomized controlled trial

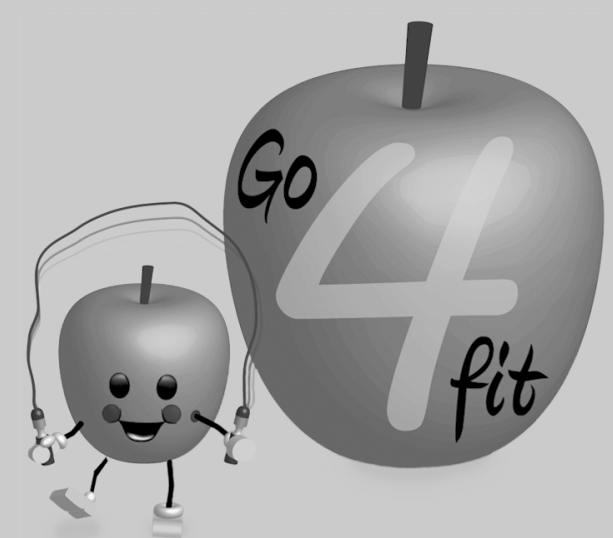

Submitted as: Gerards, S.M.P.L., Dagnelie, P.C., Gubbels, J.S., Van Buuren S., Hamers, F.J.M., Jansen, M.W.J., Van der Goot, L.O.H.M., De Vries, N.K., Sanders, M.R., Kremers S.P.J. The effectiveness of Lifestyle Triple $P$ in the Netherlands: a Randomized Controlled Trial. 


\section{Abstract}

Introduction: Lifestyle Triple $\mathrm{P}$ is a general parenting intervention which focuses on preventing further excessive weight gain in overweight and obese children. The objective of the current study was to assess the effectiveness of the Lifestyle Triple P intervention in the Netherlands.

Method: We used a randomized controlled design to test the effectiveness of the intervention. In total, 86 child-parent triads (children 4-8 years old, overweight or obese) were recruited and randomly assigned to the Lifestyle Triple P intervention or the control condition. Parents in the intervention condition received a 14-week intervention consisting of ten 90-minute group sessions and four individual telephone sessions. Primary outcome measure was the children's body composition (BMI z-scores, waist circumference, and skinfolds). Secondary outcome measures were the children's dietary behavior and physical activity level, parenting practices, parental feeding style, parenting style, and parental self-efficacy. Outcome measures were assessed at baseline and 4 months (short-term) and 12 months (long-term) after baseline. Multilevel multiple regression analyses were conducted to determine the effect of the intervention on primary and secondary outcome measures.

Results: No intervention effects were found on children's body composition. Analyses of secondary outcomes showed positive intervention effects on child behavior (soft-drink consumption (short-term), sedentary behavior (long-term), playing outside (long-term)), as well as on parenting practices (responsibility regarding physical activity (short-term) and nutrition (long-term), monitoring of food intake (long-term)), parental feeding styles (encouragement to eat (short-term)), parenting styles (psychological control (short-term)), and satisfaction with and efficacy of parenting (short-term).

Conclusion: Although the Lifestyle Triple $\mathrm{P}$ intervention showed positive effects on several child behaviors and parenting measures, no effects were visible on children's body composition. Several adjustments of the intervention content are recommended, for example including a booster session.

Trial Registration: Current Controlled Trials NTR 2555 


\section{Background}

In response to the increasing prevalence of overweight and obesity among children worldwide (WHO, 2013), childhood obesity intervention programs are being developed and evaluated on a large scale (Waters et al., 2011). The importance of involving parents in such interventions is increasingly recognized (Golley et al., 2011; Knowlden \& Sharma, 2012; Waters et al., 2011). Intervention studies involving parents predominantly aim to change so-called parenting practices, specific parenting behaviors relating to children's food and/or physical activity, for example increasing fruit availability at home. Intervention studies focusing on parenting practices have shown promising results, although effects in the longer term have been limited (Golley et al., 2011).

Another relevant aspect of parenting that influences children's weight-related health outcomes is general parenting or parenting styles (Sleddens et al., 2011). General parenting is defined as the emotional climate in which parenting takes place, encompassing parent-child interactions across situations (Darling \& Steinberg, 1993). Different dimensions of parenting can be distinguished, such as the extent to which parents respond to their children's needs (responsiveness) and the extent to which parents exert control over their children's behaviors (demandingness). Authoritative parenting (both responsive and demanding) seems to have beneficial effects on children's nutritional intake, physical activity levels, and BMI (Sleddens et al., 2011). A third relevant dimension of parenting is psychological control (Rodenburg, Kremers, Oenema, \& Van de Mheen, 2011), the extent to which parents regulate their children's behavior through psychological means, for example by guilt induction and love withdrawal.

An added value of targeting at the broader concept of parenting in addition to or instead of parenting practices, may be that this may lead to more sustained behavior change, and simultaneously, to a broad range of child outcomes, indicating a potentially large public health effect (Gerards et al., 2011). Additionally, parenting practices may be more effective when embedded in a positive parenting environment (Rodenburg, Kremers, Oenema, \& Van de Mheen, 2012; Sleddens et al., under review). Targeting parenting styles seems effective in preventing or treating childhood obesity (Brotman et al., 2012; Gerards et al., 2011; Magarey et al., 2011; Moens \& Braet, 2012), and a range of child and parental outcomes such as children's eating behaviors (Moens \& Braet, 2012), children's physical activity levels (Brotman et al., 2012), parental feeding styles (Ostbye et al., 2012), parenting practices (Ostbye et al., 2012), and general parenting (Magarey et al., 2011).

Lifestyle Triple $\mathrm{P}$ is a derivative of the Triple P Positive Parenting Program, a multilevel parenting and family support strategy (Sanders, 2012). The Lifestyle Triple P intervention aims at changing both parenting practices and general parenting styles. Its 
efficacy has been tested in a randomized controlled trial (RCT) in Australia (West et al., 2010b). Children of parents who participated in the intervention had a reduced body size and body fat content relative to the waiting-list control group (West et al., 2010b). Furthermore, the intervention increased parental confidence in managing weightrelated problem behavior and improved parenting styles.

In view of the lack of evidence-based childhood obesity prevention interventions in the Netherlands, we conducted an RCT on the effectiveness of Lifestyle Triple P in the Netherlands (Gerards et al., 2012b). The aim of the current study was to assess the effectiveness of the Lifestyle Triple $P$ intervention, primarily in terms of prevention of excessive weight gain in overweight and obese children. Primary outcome measure was the children's body composition (measured by BMI z-score, waist circumference, and skinfolds). Secondary outcome measures were the children's dietary behavior and physical activity level, parenting practices, parenting feeding style, parental style, and parenting self-efficacy.

\section{Materials and methods}

\section{Study design and setting}

The effectiveness of the Lifestyle Triple $\mathrm{P}$ intervention was tested using a parallelgroup RCT in the southern part of the province of Limburg, the Netherlands. Details of the study design and methods have been published elsewhere (Gerards et al., 2012b). The Medical Ethics Committee of the University Hospital Maastricht and Maastricht University approved the study protocol (reference number NL 31988.068.10 / MEC 103-052).

\section{Recruitment and study sample}

We enrolled 86 parent-child triads (44 intervention triad, 42 control triads). Parents of children aged between 4 and 8 years are eligible for participation if their child was considered to be overweight or obese at inclusion, based on the BMI, using the international sex- and age-specific cut-off points proposed by Cole et al. (2000). Furthermore, eligible parents were living in the southern part of Limburg, and were able to communicate in Dutch. Parents who agreed to participate and who both signed the informed consent form were included in the study. Four different recruitment strategies were used to recruit participants. First, professionals working in the Dutch youth health care system (YHC; a preventing health care system available for all children aged 0-19 years) were asked to refer parents of overweight or obese children to the Lifestyle Triple Intervention. Second, parents whose children were overweight according to the YHC medical records and other research projects were actively approached 
for participation in the intervention. Third, a mass media campaign was used to inform parents about the intervention and ask them to register for it. Finally, invitation letters were sent to parents of primary school children.

\section{Randomization}

Baseline measurements were started as soon as enough participants (a minimum of 10 parent-child triads) per site (Heerlen, Geleen or Maastricht) had been recruited. Hereafter, participating parents were randomly allocated to the intervention or control condition. The randomization scheme was generated by an independent researcher (PCD) who was not directly involved in data collection or intervention delivery, using a block size of four and sealed envelopes. The randomization was concealed to all other members of the study team. A member of the study team (SMPLG) phoned the research institute in the presence of the parents to receive the group allocation.

\section{Intervention delivery}

Parents who were assigned to the intervention condition received the Lifestyle Triple $P$ intervention, a 14-week intervention comprising ten 90-minute parental group sessions and four individual 15-30 minute telephone sessions. The intervention was led by three different Lifestyle Triple $P$ facilitators who had been trained and accredited in Triple $\mathrm{P}$ and Lifestyle Triple P. The intervention materials consisted of a parent workbook, a recipe book, and an active games booklet, all translated from English into Dutch for the current study. The Lifestyle Triple P intervention was developed by the University of Queensland in Brisbane, Australia (West \& Sanders, 2010). For a more detailed description of the intervention we refer to an earlier publication (Gerards et al., 2012b).

\section{Control condition}

Parents who were assigned to the control condition received two brochures (one on healthy nutrition and physical activity and one on positive parenting), as well as a short knowledge quiz via the Internet (sent via email) including tailored advice and suggestions for active exercises at home.

\section{Measures}

Outcome measures were assessed at baseline, at 4 months (immediately after the intervention), and at 12 months. 


\section{Primary outcome measures}

The primary outcome measure was the children's body composition, operationalized as BMI z-score, waist circumference, and biceps and triceps skinfolds, conducted by a YHC professional who was blinded for group allocation, using a standardized protocol. Weight was measured using an electronic portable scale (standardized Seca 899) to the nearest $0.1 \mathrm{~kg}$ while the child was only wearing underwear. Height was measured using a portable stadiometer (Seca 214) with an accuracy of $1 \mathrm{~mm}$. Weight and height were used to calculate Body Mass Index (BMI). BMI was then recoded into BMI zscores standardized for age and gender, based on a national reference population (i.e. the Fourth Dutch National Growth Study) (Fredriks et al., 2000). We recoded weight status into three different categories, based on BMI z-scores (Barlow, 2007): normal weight $\left(5^{\text {th }}-84^{\text {th }}\right.$ percentile), overweight $\left(85^{\text {th }}-95^{\text {th }}\right.$ percentile), obesity $\left(\geq 95^{\text {th }}\right.$ percentile). Waist circumference was measured with a flexible tape to the nearest $1 \mathrm{~mm}$. In addition, biceps and triceps skinfold thickness were measured to the nearest 0.1 $\mathrm{mm}$ using a Harpenden skinfold caliper. Each skinfold was measured three times. If the scores differed by more than $10 \%$, three extra skin-folds were measured. The median of the three or six measurements was calculated. The sum score of the biceps and triceps skinfolds was calculated and used for data analyses.

\section{Secondary outcome measures}

\section{Children's diet and physical activity level}

Dietary behavior of the child was measured using a questionnaire filled out by parents (Gubbels et al., 2011a) indicating the frequency of their child's having breakfast and having snacks, and the frequency and amount of their child's consumption of fruits, vegetables, soft drinks (including sugar-sweetened beverages), and water. The frequency was measured in days a week. The amount was measured in pieces (fruit), grams (vegetables), and glasses (soft-drink and water). The number of days and the amount were then multiplied to calculate the amount per week.

Children's physical activity level was assessed using an objective (accelerometer) and a subjective outcome measure (questionnaire for parents). Children were asked to wear an Actigraph accelerometer (Actigraph, Pensacola, Florida) for 7 consecutive days, in the week following the anthropometric measurements. Children were instructed to only remove the accelerometer at night, while they were taking a bath or shower and while they were swimming. Measurements that included at least 2 weekdays and 1 weekend day (total wearing time $\geq 600$ minutes) were considered valid and were used in the analyses. Periods of $\geq 90$ minutes of non-wearing time (defined as 
consecutive zero counts) were removed from the data (Choi, Liu, Matthews, \& Buchowski, 2011). The time interval or epoch was set at $15 \mathrm{sec}$. The thresholds proposed by Evenson et al. (2008) were used to distinguish different intensities of physical activity: sedentary behavior ( $\leq 25$ counts per epoch), light physical activity (26 - 573 counts per epoch), moderate physical activity (574 - 1002 counts per epoch), and vigorous physical activity ( $\geq 1003$ counts per epoch). We combined moderate and vigorous physical activity into one category.

In addition, parents were asked to indicate the frequency (days a week) and duration (number of hours) of several physical activity behaviors (Gubbels et al., 2011a): TV watching, computer games (combined into one scale, i.e., sedentary behavior), playing outside, sports club attendance, and use of active transport (walking/cycling to and from school). The number of days and duration were multiplied to calculate the number of hours spent on a particular activity per week.

\section{Parenting practices}

Parenting practices were measured using two scales of the Child Feeding Questionnaire (CFQ; see Table 8.1 for detailed information about the scales). The responsibility regarding nutrition scale consisted of two items assessing parents' perceptions of their responsibility for their child's feeding. The monitoring food intake scale assessed the extent to which parents oversaw their child's eating behavior.

In addition, we used CFQ items which were converted to the physical activity context, also called the 'Physical Activity-Related Parenting Questionnaire' (Gubbels et al., 2011a): responsibility regarding physical activity (parents' perception of their responsibility for their child's physical activity level), and monitoring physical activity (the extent to which parents check their child's physical activity level).

\section{Feeding styles}

The Parental Feeding Style Questionnaire (Wardle et al., 2001) was used to measure four different feeding styles: instrumental feeding (using food as a reward), emotional feeding (feeding in response to emotional distress), encouragement to eat (encouraging food variety and interest in food), and control over eating (parental restrictions; see Table 8.1).

\section{Parenting styles}

A validated Dutch version of the Child Rearing Practices Report (Dekovic, 1989) was included to assess parents' child-rearing attitudes, values, behaviors, and goals. The questionnaire can be used to distinguish two different parenting styles: authoritative parenting and authoritarian parenting (see Table 8.1). Items on the authoritarian scale 
reflect 'the frequent use of physical punishment, verbal reprimands, prohibitions, discouragement of the child's expression, emphasis on fear of external consequences of transgression and strict supervision of child' (Dekovic, 1989). The authoritative scale consists of items 'indicating the emphasis on inductive methods, reasoning with the child, appreciation of the child's accomplishments, fostering the child's individuality, and encouraging open communication between parents and the child regarding both positive and negative feelings' (Dekovic, 1989). Additionally, we included items from a Dutch version of the validated psychological control scale (Barber, 1996).

\section{Efficacy of and satisfaction with parenting}

The validated 'Being a Parent Scale' (Johnston \& Mash, 1989) was used to assess parenting self-esteem, which consists of parental self-efficacy and their satisfaction derived from parenting. All individual items were combined into a scale measuring parenting self-esteem (total score on the Being a Parent Scale) (Johnston \& Mash, 1989).

\section{Demographics}

A range of demographics was included in the baseline questionnaire. Child characteristics assessed included gender and date of birth. Parental characteristics included country of birth of mother and father (recoded into Netherlands vs. other), work situation of mother and father (hours of paid work per week), and educational level of mother and father. Educational level was recoded into low (primary school, lower vocational education, and lower general secondary education), medium (intermediate vocational education, higher general secondary education, and university preparatory education), and high (higher vocational education and university). Finally, the marital status of the parents was recoded into married or living together vs. other.

At baseline, weight and height of parents were measured (in order to calculate their BMI), using the same standardized procedures as described above for the children. If the parents were not willing to undress and thus were fully clothed, $1 \mathrm{~kg}$ was subtracted from the body weight (Visscher \& Seidell, 2004).

\section{Process evaluation}

Parental satisfaction with the intervention was measured by process evaluation items in the questionnaire at 4 months, for example: 'How would you rate the intervention on a scale from $0-10$ ?' 
Table 8.1: Overview of parenting scales

\begin{tabular}{|c|c|c|c|}
\hline Scale & $\mathrm{N}$ items & $\begin{array}{l}\text { Cronbach's } \\
\text { Alpha }\end{array}$ & Example of items \\
\hline \multicolumn{4}{|l|}{ Parenting practices $^{a}$} \\
\hline Monitoring food intake & 4 & 0.76 & $\begin{array}{l}\text { How often do you keep track of the sweets and } \\
\text { the snack food that your child eats? }\end{array}$ \\
\hline Responsibility regarding nutrition & 2 & 0.58 & $\begin{array}{l}\text { How often are you responsible for deciding your } \\
\text { child's portion sizes? }\end{array}$ \\
\hline Monitoring physical activity & 2 & 0.67 & $\begin{array}{l}\text { How often do you keep track of the amount of } \\
\text { physical activity your child engages in? }\end{array}$ \\
\hline $\begin{array}{l}\text { Responsibility regarding physical } \\
\text { activity }\end{array}$ & 2 & 0.91 & $\begin{array}{l}\text { How often are you responsible for deciding } \\
\text { whether your child gets enough physical } \\
\text { activity? }\end{array}$ \\
\hline \multicolumn{4}{|l|}{ Feeding styles $^{b}$} \\
\hline Control over eating & 10 & 0.73 & $\begin{array}{l}\text { I allow my child to choose which foods to have } \\
\text { for meals. }\end{array}$ \\
\hline Instrumental feeding & 4 & 0.69 & $\begin{array}{l}\text { In order to get my child to behave him/herself I } \\
\text { promise him/her something to eat. }\end{array}$ \\
\hline Emotional feeding & 5 & 0.83 & $\begin{array}{l}\text { I give my child something to eat to make } \\
\text { him/her feel better when he/she is feeling } \\
\text { upset. }\end{array}$ \\
\hline Encouragement to eat & 8 & 0.76 & $\begin{array}{l}\text { I encourage my child to taste foods that he/she } \\
\text { has not tasted before }\end{array}$ \\
\hline \multicolumn{4}{|l|}{ General parenting } \\
\hline Authoritative parenting ${ }^{c}$ & 12 & 0.61 & $\begin{array}{l}\text { I respect my child's opinion and encourage } \\
\text { him/her to express it. }\end{array}$ \\
\hline Authoritarian parenting $^{c}$ & 12 & 0.62 & $\begin{array}{l}\text { I believe children should not have secrets from } \\
\text { their parents. }\end{array}$ \\
\hline Psychological control $^{\mathrm{C}}$ & 8 & 0.60 & $\begin{array}{l}\text { I avoid looking at my child if he/she does not see } \\
\text { things my way. }\end{array}$ \\
\hline $\begin{array}{l}\text { Efficacy of and satisfaction with } \\
\text { parenting }^{d}\end{array}$ & 16 & 0.76 & Sometimes I feel I'm not getting anything done. \\
\hline
\end{tabular}

${ }^{a} 5$-point Likert scale form disagree to agree, ${ }^{b} 5$-point Likert scale from never to always, ${ }^{c} 5$-point Likert scale from completely disagree to completely agree, ${ }^{d} 6$-pont Likert scale from strongly disagree to strongly agree

\section{Statistical analysis}

SPSS 19.0 was used for the analyses. In all analyses, $\mathrm{p}$-values $<0.05$ were considered statistically significant. Descriptive statistics were calculated to describe demographics. The internal consistency of the scales was determined by calculating Cronbach's alpha. We calculated effect sizes (ES; Cohen's d) by dividing the change in the outcome measures by the pooled standard deviation of the baseline scores of the study group (Cohen, 1992). Effect sizes were interpreted using the classification defined by Lipsey: 
small effect (ES $\leq 0.32$ ), medium effect (ES 0.33 - 0.55), or large effect (ES $\geq 0.56$ ) (Lipsey, 1990).

Multilevel multiple regression analyses were conducted to determine the effect of the intervention on changes in primary and secondary outcome measures, at 4 and 12 months (short-term and long-term), adjusting for the multilevel structure introduced by the delivery of the intervention in groups. Random coefficients (a random intercept on the group level and a random slope for condition (intervention vs. control) on the group level) were entered using a forward procedure, and were retained in the final model if the -2 log likelihood of the model changed significantly compared to the previous model (Likelihood ratio test).

Subsequently, models were corrected for relevant confounders (regression coefficient of condition changes $>10 \%$ when the confounder is included in the model), i.e. child's age, gender, mother's country of birth, mother's educational level (low, medium, high), mother's employment status (hours per week), and marital status (married or partners living together vs. not). Missing values on covariates were imputed by the group mean (linear variables) or the median (categorical variables).

We performed complete-case analyses using all available data. In addition, we used multiple imputation to assess the impact of missing responses (Van Buuren, 2012), using $m=10$. Whereas the complete case analyses are the main focus of the paper, results of the regression analyses in which the multiple imputation approach was used to treat missing values are depicted in the supplement, see pages 175-177.

\section{Results}

\section{Response}

Of the 86 families who were randomized and underwent baseline anthropometric measurements, 76 (88\%) filled out the baseline questionnaire for the child, and 74 children (86\%) had valid baseline accelerometer data, see Figure 8.1. At 4 months after baseline, 71 families (83\%) attended the anthropometric measurements. Sixty-two families (72\%) filled out the 4-months questionnaire, and 56 children (65\%) had valid accelerometer data. Twelve months after baseline, 69 families (80\%) (including 59 mothers and 29 fathers) attended the anthropometric measurements. The final questionnaire was filled out by 62 families (72\%) and 56 children (65\%) had valid accelerometer data. Drop-out with regard to demographics was non-selective.

\section{Implementation of the intervention}

The majority of the group sessions which were planned actually took place: only 2 of the 60 group sessions were cancelled due to holidays or due to absence of the majority 
of the parents. Thirty percent ( $N=13$; nonselective regarding demographics) of the families who were assigned to the intervention condition did not attend any intervention session at all. Of the parents who did visit at least one group session, $81 \%(N=25)$ were present at 5 or more sessions. Furthermore, parents who were attending the group sessions had an average of at least 2 telephone sessions. Parents rated the value of the intervention as 7.6 on a 10 -point scale $(84 \% \geq 7)$.

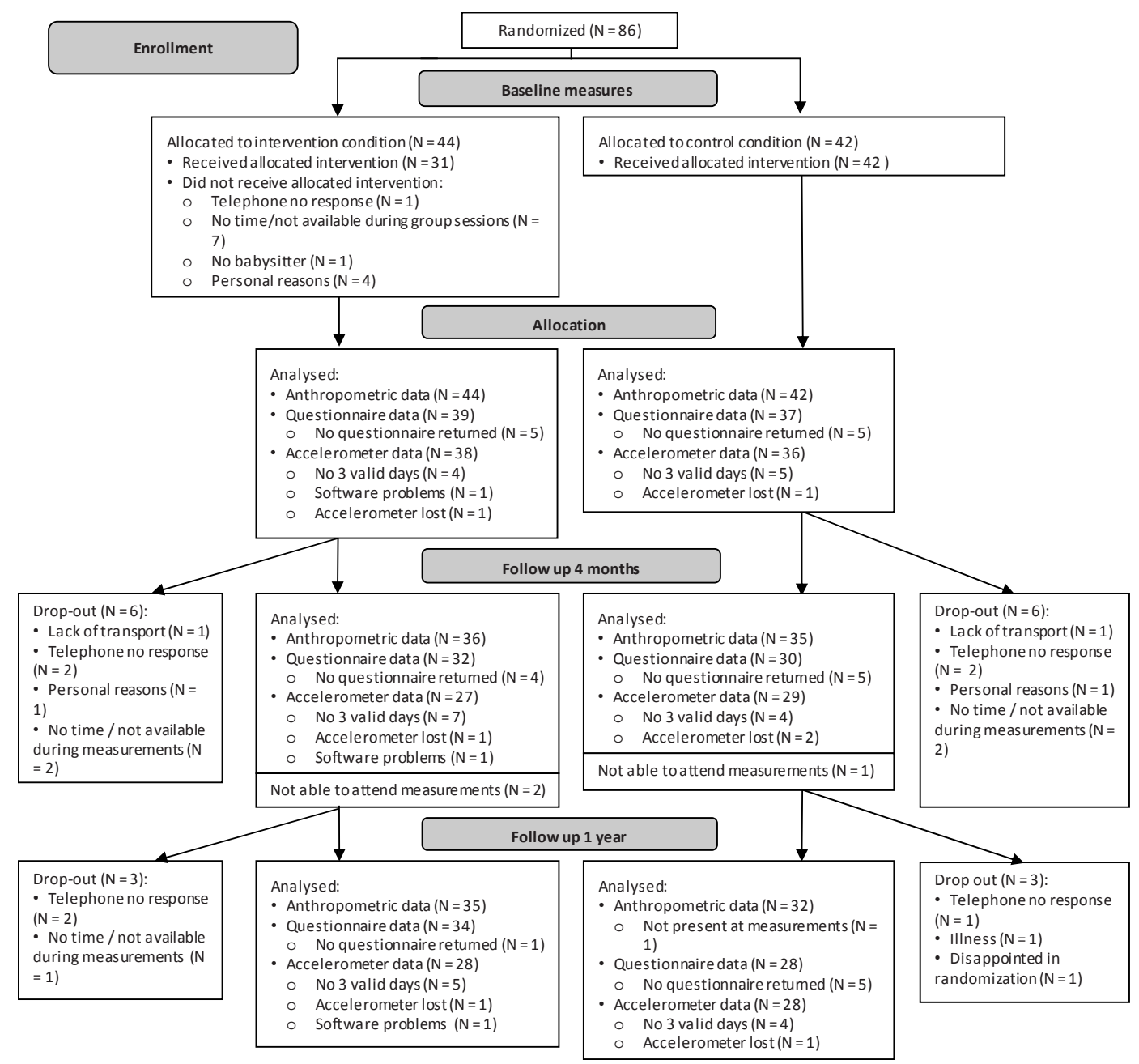

Figure 8.1: Flow chart of participants 


\section{Baseline data}

The mean age of the children was $7.2( \pm 1.4$ ) years (see Table 8.2 ). More than half (56\%) of the children were female and $63 \%$ of the children were obese. The majority of the parents was married or living together (87\%), and was born in the Netherlands ( $78 \%$ of the mothers and $72 \%$ of the fathers). The majority of the parents had a medium educational level ( $43 \%$ of the mothers and $37 \%$ of the fathers) and were overweight or obese ( $61 \%$ of the mothers and $78 \%$ of the fathers). Mothers in the intervention condition had a higher BMI than those in the control condition (30.19 vs. 26.29).

\section{Outcomes}

\section{Primary outcome measure}

We did not find any significant intervention effects on children's BMI z-score, waist circumference, or skinfold thickness at 4 and 12 months after baseline (see Table 8.3).

\section{Secondary outcome measures}

Children's nutrition and physical activity level

Regarding children's physical activity level and dietary behavior, all differences between the intervention and control condition with at least a medium effect size were in favor of the intervention condition (Table 8.4). At 4 months, a significant intervention effect (with a large effect size) was found for soft-drink consumption: intervention children decreased their consumption of soft drinks over time, while control children increased their consumption. At 12 months, statistically significant intervention effects were found on sedentary activity and playing outside: children in the intervention condition decreased their time spent on TV viewing and computer games more than children in the control condition, while intervention children spent more time playing outside than control children. Other nutrition and physical activity measures did not change significantly, although the differences in time spent playing outside (shortterm) and use of active transport (long-term) almost reached significance $(P=0.063$ and $\mathrm{P}=0.058$ respectively). 
Table 8.2: Baseline characteristics

\begin{tabular}{|c|c|c|c|c|c|c|}
\hline & \multirow[t]{2}{*}{ Variable } & & \multicolumn{2}{|c|}{ Intervention ( $N=44)$} & \multicolumn{2}{|c|}{ Control $(\mathrm{N}=42)$} \\
\hline & & & $\mathrm{N}(\%)$ & Mean \pm SD & $\mathrm{N}(\%)$ & Mean \pm SD \\
\hline \multirow[t]{8}{*}{ Child } & Gender & Male & $19(43.2)$ & & $19(45.2)$ & \\
\hline & & Female & $25(56.8)$ & & $23(54.8)$ & \\
\hline & Age & & & $7.14 \pm 1.55$ & & $7.29 \pm 1.31$ \\
\hline & BMI z-score & & & $1.82 \pm 0.83$ & & $1.87 \pm 0.74$ \\
\hline & BMI score & & & $20.37 \pm 2.79$ & & $20.67 \pm 3.12$ \\
\hline & Weight status & Normal & $8(18.2)$ & & $5(11.9)$ & \\
\hline & & Overweight & $8(18.2)$ & & $11(26.2)$ & \\
\hline & & Obesity & $28(63.6)$ & & $26(61.9)$ & \\
\hline \multirow[t]{2}{*}{ Parents $^{1}$} & Marital status & Married or living together & $33(84.6)$ & & $33(89.2)$ & \\
\hline & & Other & $6(15.4)$ & & $4(10.8)$ & \\
\hline \multirow[t]{10}{*}{ Mother $^{1,2}$} & Country of birth & Netherlands & $33(84.6)$ & & $26(70.3)$ & \\
\hline & & Other & $6(15.4)$ & & $11(29.7)$ & \\
\hline & Educational level & Low & 7 (17.9) & & $10(27.0)$ & \\
\hline & & Medium & $20(51.3)$ & & $12(32.4)$ & \\
\hline & & High & $12(30.8)$ & & $15(40.5)$ & \\
\hline & Employment & (hours per week) & & $17.55 \pm 12.93$ & & $15.94 \pm 15.97$ \\
\hline & BMI & & & $30.19 \pm 6.71$ & & $26.92 \pm 4.38$ \\
\hline & Weight status & Normal & $10(26.3)$ & & $17(44.7)$ & \\
\hline & & Overweight & $8(22.2)$ & & $13(34.2)$ & \\
\hline & & Obesity & $18(47.4)$ & & $8(21.1)$ & \\
\hline \multirow[t]{10}{*}{ Fathers ${ }^{1,3}$} & Country of birth & Netherlands & 30 (76.9) & & $25(67.5)$ & \\
\hline & & Other & 7 (17.9) & & $10(27.0)$ & \\
\hline & Educational level & Low & 7 (17.9) & & $8(21.6)$ & \\
\hline & & Medium & $14(35.9)$ & & $14(37.8)$ & \\
\hline & & High & $13(33.3)$ & & $12(32.4)$ & \\
\hline & Employment & (hours per week) & & $31.99 \pm 15.08$ & & $25.11 \pm 17.85$ \\
\hline & BMI & & & $30.65 \pm 5.17$ & & $28.68 \pm 5.16$ \\
\hline & Weight status & Normal & $5(19.2)$ & & $6(26.1)$ & \\
\hline & & Overweight & 7 (26.9) & & $10(43.5)$ & \\
\hline & & Obesity & $14(53.8)$ & & $7(30.4)$ & \\
\hline
\end{tabular}

Note: ${ }^{1} 39$ parents in the intervention condition and 37 parents in the control condition filled out the baseline questionnaire, ${ }^{2} 38$ mothers in the intervention condition and 38 mothers in the control condition attended the anthropometric measurement sessions, ${ }^{3} 26$ fathers in the intervention condition and 23 fathers in the control condition attended the anthropometric measurement sessions 
168 CHAPTER 8

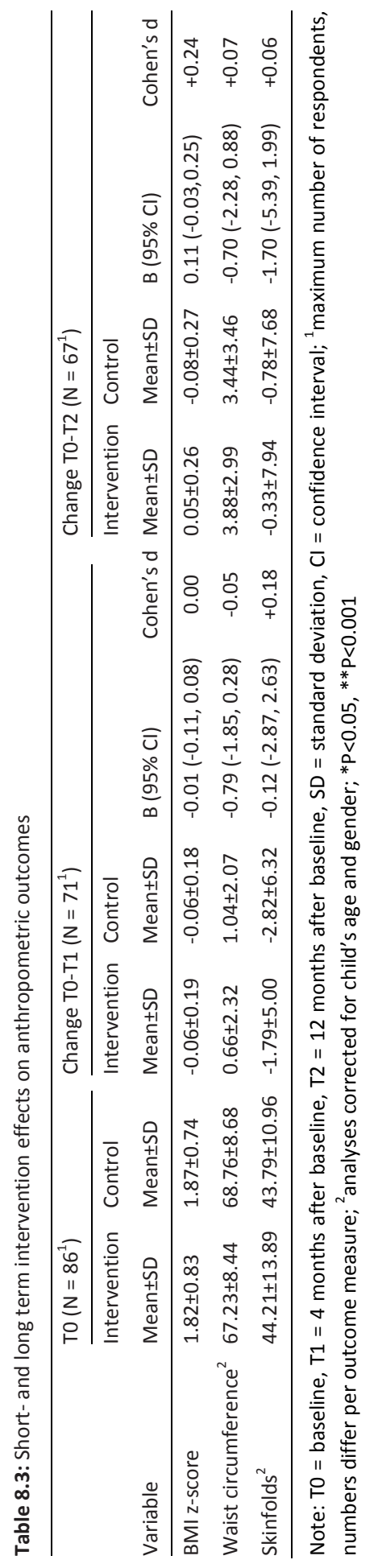




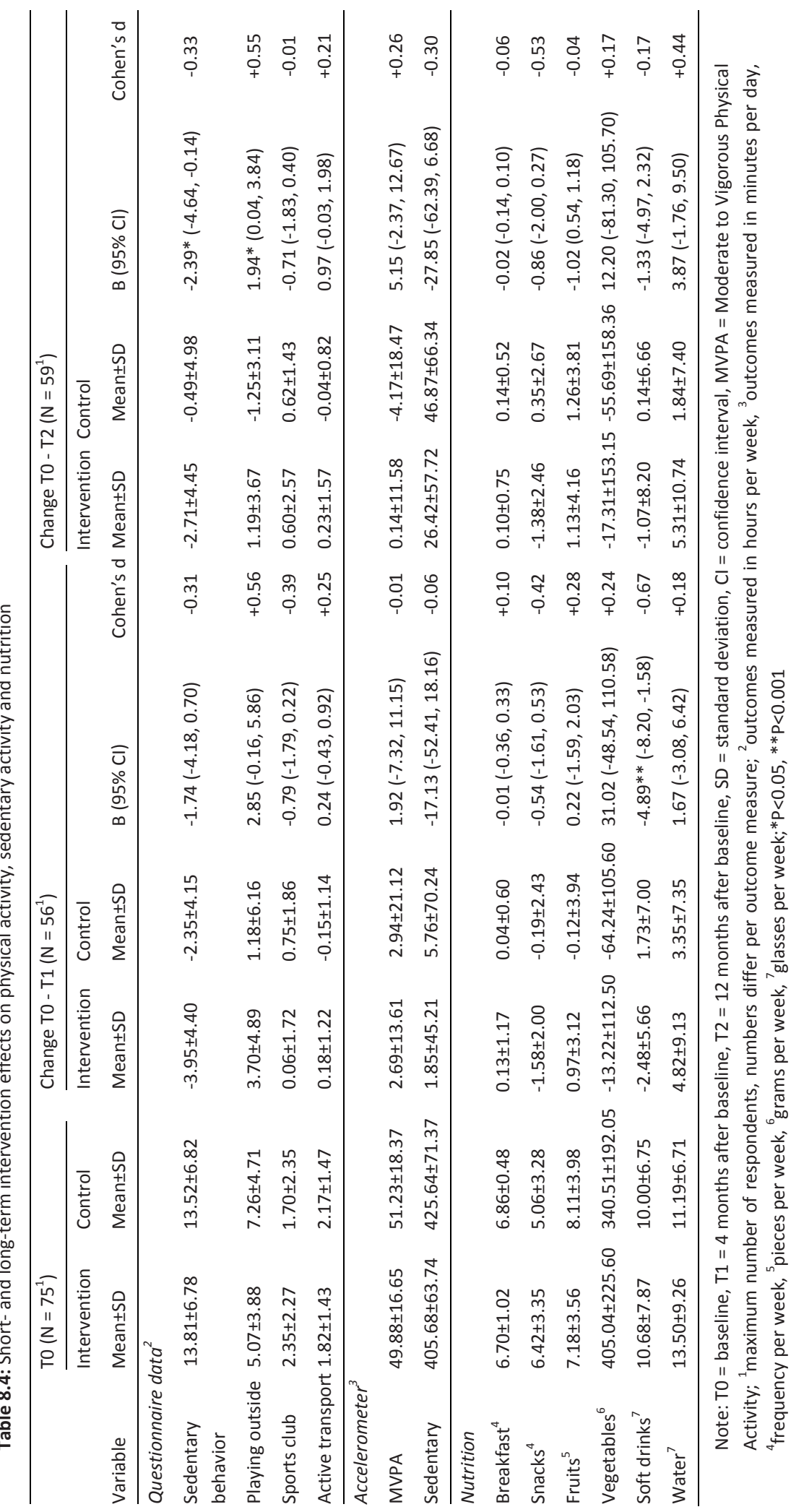




\section{Parenting behaviors}

All statistically significant differences regarding parenting outcomes were in favor of the intervention condition (Table 8.5). Parents in the intervention condition slightly increased their responsibility regarding physical activity at 4 months follow-up, whereas parents in the control condition reduced this. At 12 months, intervention effects were visible on monitoring of food intake (medium effect size) and responsibility regarding nutrition (small effect size): intervention parents increased their monitoring of the child's food intake compared to baseline and felt equally responsible compared to baseline, while control parents reduced their monitoring of the child's food intake and felt less responsible for the child's nutrition.

Change in the amount of encouragement to eat differed significantly between the groups at 4 months (medium effect size): intervention parents increased their encouragement of food variety and interest in food, while control parents did not change. This difference in change was no longer visible at 12 months after baseline. Differences in control over eating, instrumental feeding, and emotional feeding were not statistically significant.

At 4 months, intervention effects regarding parenting styles were visible on psychological control (medium effect size) and efficacy of and satisfaction with parenting (large effect size): intervention parents reduced their psychological control and increased their perceived efficacy and satisfaction regarding parenting compared to baseline, while the opposite was seen for control parents. These intervention effects were no longer statistically significant at 12 months. No significant differences in change were found with regard to authoritative and authoritarian parenting. 


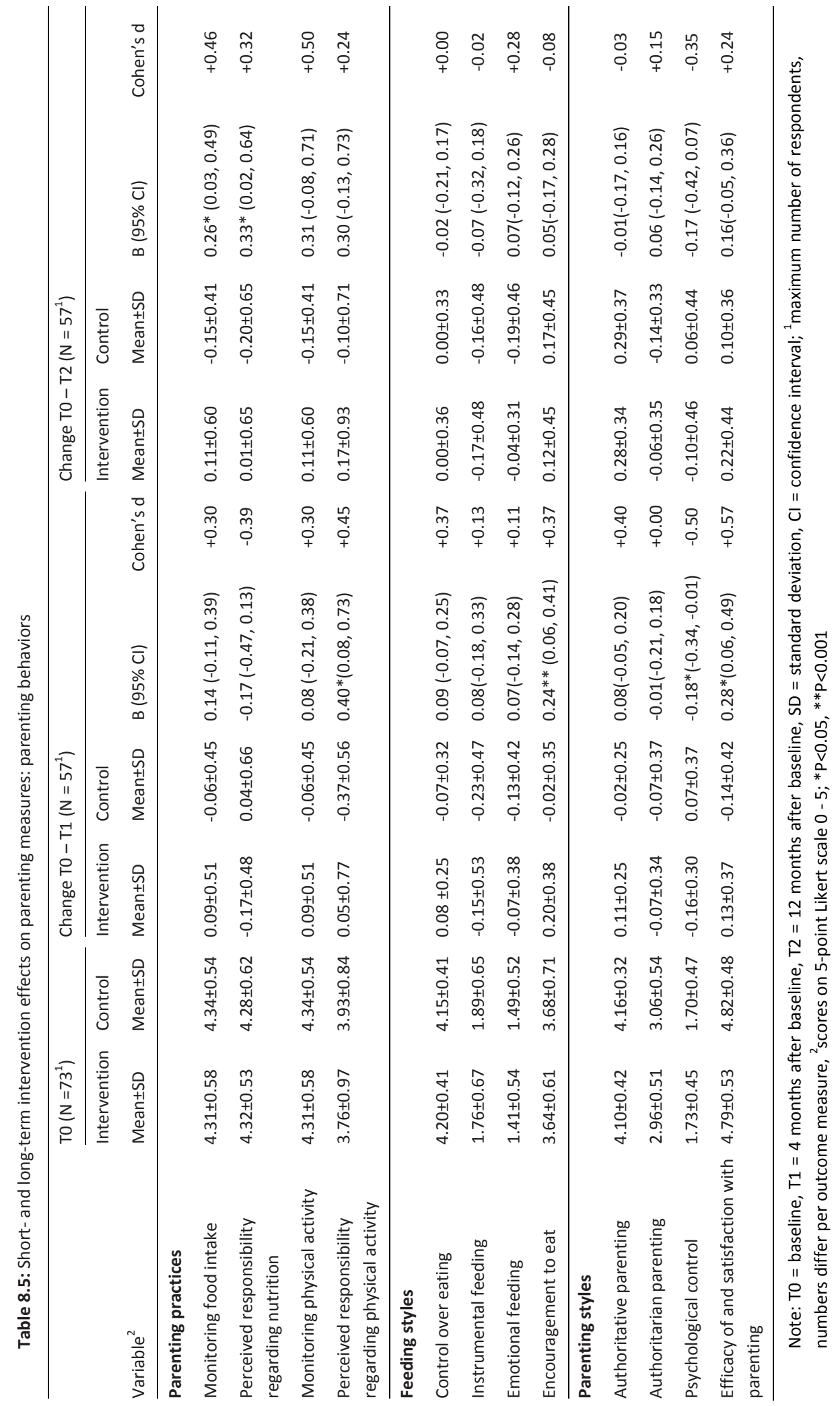




\section{Discussion}

The current study evaluated the effectiveness of a general parenting intervention, Lifestyle Triple P, aimed at preventing childhood obesity. Lifestyle Triple P is a derivative of the Triple P Positive Parenting Program (Sanders et al., 2003), a multi-level parenting and family support strategy. Results of the current study showed no beneficial effect of this intervention on the primary outcome measure of children's BMI z-score, waist circumference, and skin-folds, compared to the control condition, neither immediately after the intervention (4 months) nor in the longer term (12 months). We did find short-term intervention effects on soft-drink consumption, responsibility regarding physical activity, encouragement to eat, psychological control, and efficacy and satisfaction with parenting. Longer-term intervention effects were found on time spent on sedentary activity (TV viewing and computer games), time spent on playing outside, monitoring food intake, and responsibility regarding nutrition.

Several explanations can be given for the apparent lack of effects on the primary outcome measure. The Lifestyle Triple $\mathrm{P}$ intervention is a behavioral intervention which aims to change behavioral determinants (parenting behavior), in order to influence behavior (children's nutrition and physical activity level), thereby finally affecting children's weight development. Thus, the causal chain in our effectiveness study is rather long. This could explain why we did find some changes in behavior as a result of the intervention, whereas these changes did not, or at least not yet, result in improvements in children's weight status and body composition. It is conceivable that if these behavioral changes are sustained over a longer time (> 12 months), changes in children's weight development may still occur.

Some intervention components could however be further optimized. The recipes provided were found to be quite difficult for parents and not always appropriate to the Dutch eating habits. Furthermore, parents indicated that they would have liked a booster session (for example after 6 months) to refresh their knowledge and skills. Although Golan and colleagues (2006) found better outcomes when parents were treated alone and children were not included in intervention sessions, it is worth considering whether children should be involved in the intervention.

Results of intervention approaches similar to Lifestyle Triple $\mathrm{P}$ to date have been mixed; some studies found promising effects (Brotman et al., 2012; Magarey et al., 2011; Moens \& Braet, 2012; Robertson et al., 2008; Stark et al., 2011; West et al., 2010b), while others found no effect on primary outcomes (Ostbye et al., 2012; Golley et al., 2007a; Moens \& Braet, 2012). The efficacy of the Lifestyle Triple P intervention was tested previously in an RCT with a waiting-list control condition, by West and colleagues (2010b). These authors reported positive intervention effects on weightrelated outcomes. However, comparison between this and our study is hampered by 
differences in the control conditions (waiting-list control vs. control intervention). Trials using waiting-list control conditions are more likely to show between group differences, but trials using a control intervention may provide an underestimation of the total effect of the intervention (Foster \& Little, 2012). Also, the study sample used by West et al. was, on average, more obese than our sample (mean BMI z-scores 2.11 vs. mean BMI z-scores 1.85), which probably gives more room for improvement on BMI measures. Furthermore, the children in the study of West et al. were generally older (8.5 years vs. 7 years) which limits study comparability, due to differences in parental influence and differences in growth patterns at various ages. Finally, the Australian study was implemented as an efficacy study, while in the Dutch trial we tried to implement in the real life situation, which may have led to less significant study results (Koepsell, Zatzick, \& Rivara, 2011).

\section{Strengths and limitations}

Strengths of the current study include the RCT design with concealed group allocation, long-term follow-up (12 months after baseline), and high retention rates, which were similar for both conditions ( $80 \%$ at 12 months). Furthermore, the quality of the measurements was high: all anthropometric measurements were standardized and performed by the same trained research assistant who was blinded to the research condition, preventing measurement bias. Furthermore, we used validated questionnaires and accelerometry for objective physical activity assessment. The intervention was based both on theory and evidence, and was highly appreciated by the parents who participated in the group sessions. Quality of the delivery of the intervention was assured by training and accreditation of facilitators, ongoing supervision, and manuals.

Limitations include the relatively small sample size was relatively small, although we achieved enough power according to our sample size calculation (see Gerards et al. 2012b). We put a lot of effort into the recruitment of parents (Gerards et al., 2012a) and recruitment took longer than planned. Recruiting parents has been shown to be a challenge in other intervention studies as well (Knowlden \& Sharma, 2012).

Another limitation concerns the presence of missing values, which limited the possibility to conduct intention-to-treat analyses. In order to overcome this problem, we applied a multiple imputation approach (Van Buuren, 2012) to treat missing values. Since the use of multi-level analysis of multiple imputation data is still underinvestigated, more research is necessary in this area in order to provide reliable estimates (Van Buuren, 2012). Consequently, we performed multiple linear regression analyses on the data in which the multiple imputation approach was used to treat 
missing values. This approach yielded effects in the same direction, but the effects were non-significant under listwise deletion, see the supplement, on pages 175-177.

\section{Conclusion}

Although the Lifestyle Triple P intervention showed positive effects on different child behaviors and parenting measures, no effects were visible on children's BMI z-scores or body composition 4 and 12 months after baseline. 


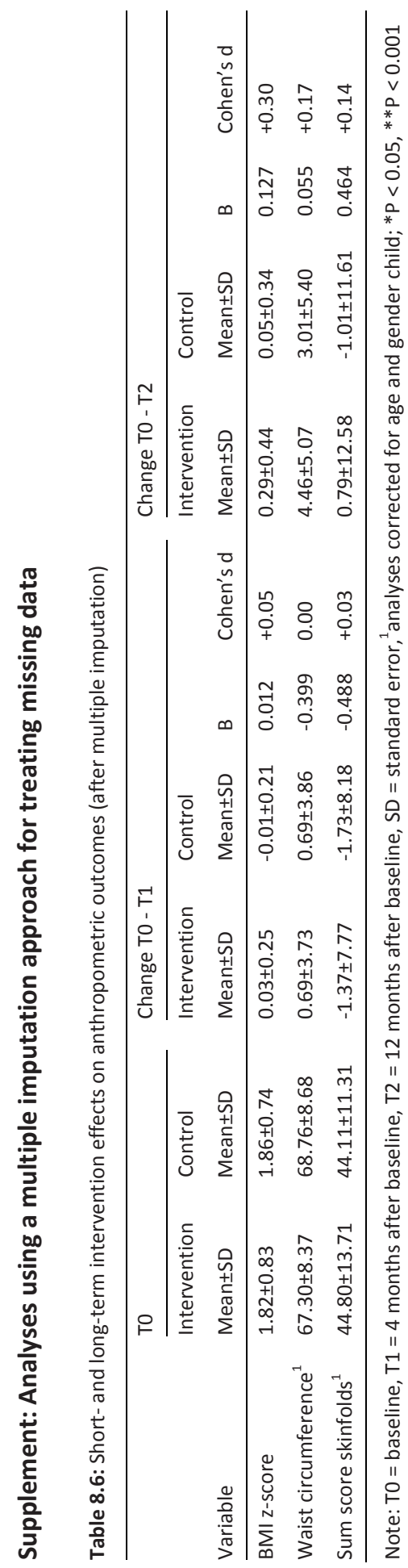




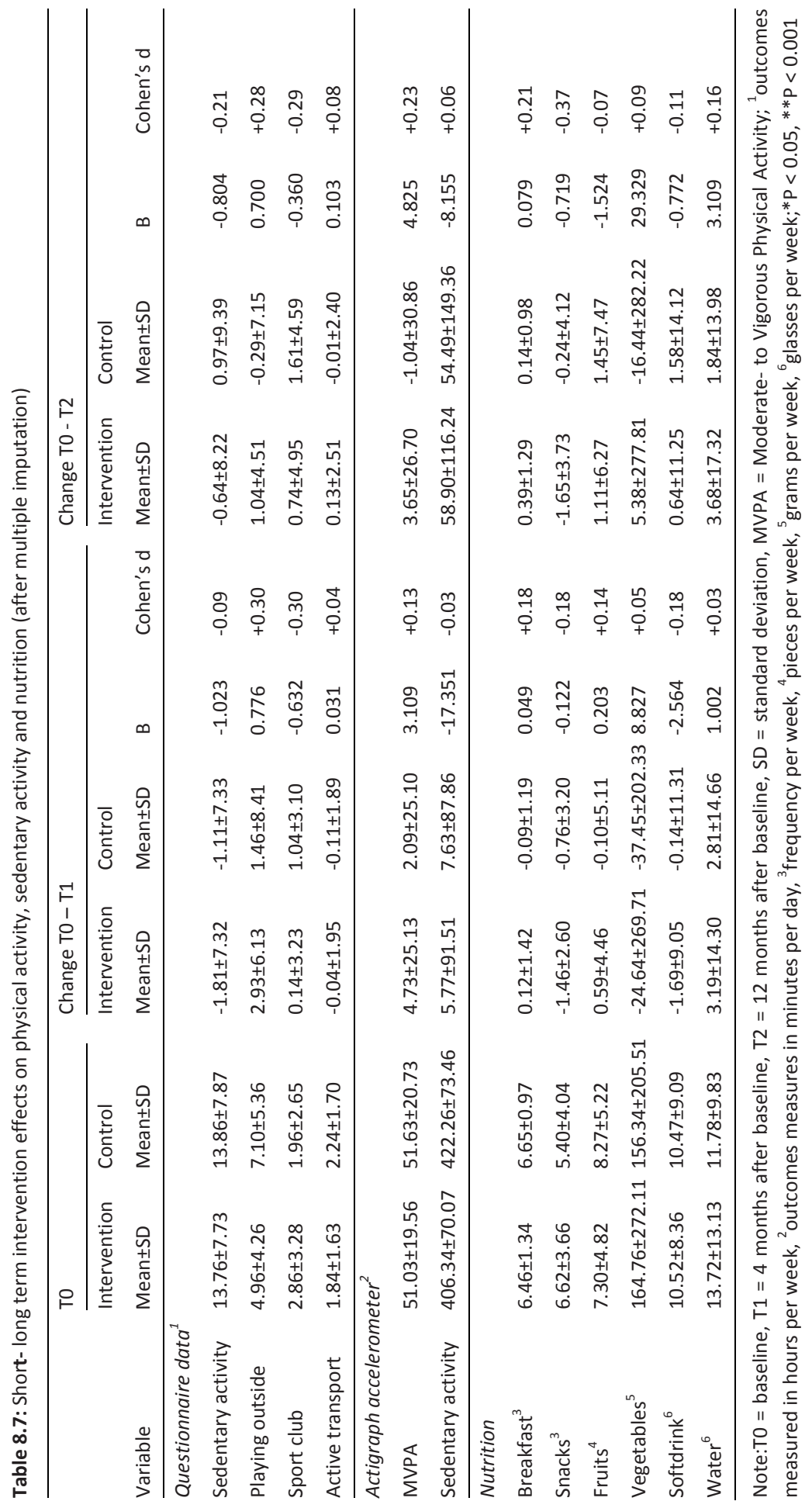




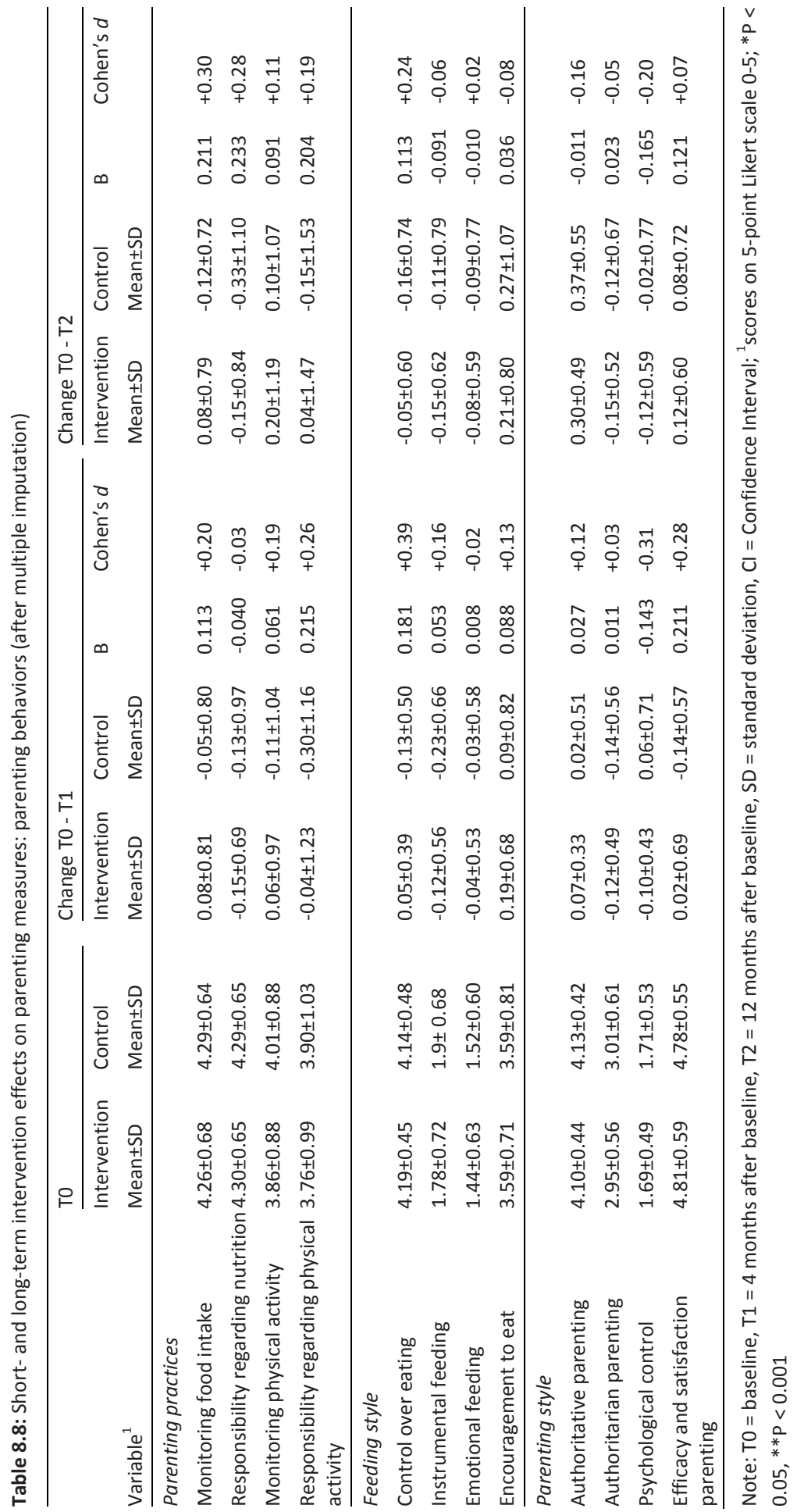



CHAPTER 9

General discussion

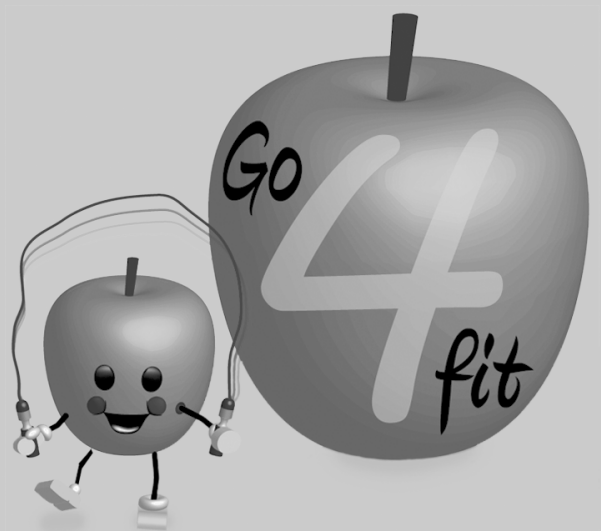


The current thesis describes the rationale, implementation, and evaluation of the Lifestyle Triple P intervention aimed at parents of 4-8 year-old overweight children in the Netherlands. This final chapter first summarizes the main findings of the studies, then considers methodological issues and describes implications for practice and research. Finally, it draws some general conclusions.

\section{Main Findings}

The existing literature regarding the relationship between general parenting (i.e., the emotional climate) and children's weight-related outcomes was evaluated in chapter 2 , to provide a theoretical foundation. The literature review included a total of 36 studies. Overall, the included studies provide evidence that children who are raised in authoritative homes (i.e. by parents who are both responsive and demanding) have a more healthy diet, are more physically active and have lower BMI scores than children who are raised with other parenting styles. Findings of interaction studies showed that general parenting can have a differential impact on children's weight-related health behaviors, depending on the characteristics of the children and parents.

Chapter 3 provides an overview of intervention studies addressing general parenting in order to prevent or manage childhood obesity. Seven studies were identified, which represented four different general parenting programs complemented with lifestyle components. These studies showed small to moderate intervention effects on one or more weight-related outcome measures. The review provides evidence that the promotion of authoritative parenting is a valuable addition to the focus on parenting practices in the prevention and management of childhood obesity.

The aim of the study reported on in chapter 4 was to evaluate whether parents are able to accurately perceive their child's weight status (normal weight, overweight or obesity) at age 5, and what factors predict accurate weight status perception. We also investigated the predictive value of accurate weight status perception in explaining children's longitudinal weight development up to the age of 9. For this purpose, we used data of the KOALA Birth Cohort Study. The majority of parents of overweight children underestimated their child's weight status. Children of parents who accurately perceived their child's overweight status at ages 2 and 5 years subsequently developed a higher BMI over time. Parental awareness as such may thus not be sufficient for subsequent effective weight management by parents.

Chapter 5 assessed the test-retest reliability and construct validity of the Dutch translation of the Lifestyle Behavior Checklist (LBC), a measure to indicate parental perceptions of children's behavioral problems related to overweight and obesity (problem scale), and parental self-efficacy in dealing with these problems (confidence). As 
part of testing the construct validity, correlation tests were performed between the confidence and problem scales on the one hand, and general parenting measures (nurturance, restrictiveness, and psychological control) on the other. The LBC was found to be a reliable and reasonably valid questionnaire. The instrument can be used to assess intermediate outcomes in future evaluation studies.

In the qualitative study presented in chapter 6, 16 Dutch youth health care (YHC) professionals were interviewed in order to identify barriers to referring parents of overweight children to an obesity prevention intervention. An intervention implementation model was used as the framework for conducting, analyzing, and interpreting the interviews. This model identified five categories of factors influencing the referral procedure: characteristics of the socio-political environment, the YHC organization, the YHC professionals, the innovation, and the parents. The most important aspects impeding optimal referral seemed to be the perceived characteristics of the parents, i.e. lack of awareness and resistance. YHC professionals appeared to lack sufficient skills and self-efficacy towards discussing weight issues with parents of overweight children.

Chapter 7 provides a detailed overview of the study protocol of a randomized controlled trial (RCT) on the effectiveness of the Lifestyle Triple P intervention, a parent-focused group program simultaneously addressing three topics: nutrition, physical activity, and positive parenting. The aim of the study was to test the effectiveness of this intervention program when applied to Dutch parents of overweight and obese children aged 4-8 years. According to the study protocol, parents in the intervention condition received a 14-week intervention in which they learned a range of strategies related to nutrition, physical activity, and positive parenting. Parents in the control condition received brochures, web-based tailored advice, and suggestions for active play. Measurements were conducted at baseline, 4 months and 12 months.

Chapter 8 presents the short-and long-term results of the above RCT. No intervention effects were found on the primary outcome measures of BMI z-scores, waist circumference, and skin-fold thickness. Significant short-term intervention effects were found on several secondary outcome measures: soft-drink consumption, responsibility regarding physical activity, encouragement to eat, psychological control, and efficacy and satisfaction with parenting. Furthermore, longer-term intervention effects were found on time spent on sedentary activity (TV viewing and computer games), time spent on playing outside, monitoring food intake, and responsibility regarding nutrition. 


\section{The Lifestyle Triple P RCT: methodological considerations}

A randomized controlled trial was chosen as the most appropriate design for testing the effectiveness of the Lifestyle Triple P intervention. This design is considered to be the 'gold standard' for testing the effectiveness of interventions (Campbell et al., 2000). Some methodological issues related to this RCT are outlined below.

\section{Recruitment}

A well-known challenge in implementing childhood obesity interventions is the recruitment of participants (Knowlden \& Sharma, 2012). The current trial was no exception in this respect. A problem in recruiting these children and their parents is that parents of overweight children often underestimate their child's weight status (see chapter 4). And parents who do recognize their child as overweight may not see the need to participate in overweight intervention studies (Warschburger \& Kroller, 2012). In the interviews with YHC professionals (see chapter 6), they indicated that they perceived difficulties in discussing weight issues with parents, as they often encountered much resistance. Unfortunately, time constraints prevented us from investigating the parental views regarding this problem.

Our recruitment strategy consisted initially of a referral procedure via the Dutch youth health care system: YHC professionals were asked to refer parents of overweight children to the intervention, as outlined in chapter 4 . However, due to the disappointingly low number of parents who were actually being referred, we adjusted our approach and used several different recruitment strategies. First of all, the inclusion criteria were widened: initially, only parents of overweight children aged 4 years were eligible for participation: this was expanded to include parents of children who were already obese and up to 8 years old. Furthermore, the recruitment strategy was adjusted: via the YHC, we also directly approached parents who were eligible according to their child's medical records. Additionally, parents were recruited via other pathways than the YHC: mass media materials (a poster, a brochure, advertisements in newspapers and a website) were developed to inform parents about the intervention and to offer them opportunities to register for the intervention. In addition, invitation letters were sent to all parents of primary school children and parents rejected for participation in other study cohorts. The active recruitment strategy through the YHC proved to be the most effective in recruiting parents, confirming an earlier report which indicated that active recruitment strategies produced higher inclusion rates than passive recruitment strategies (Raynor et al., 2009).

It took us 15 months (from December 2010 until February 2012) to recruit the final study sample ( $N=86$ families). In total, $22 \%$ of the included children were overweight and $63 \%$ were obese. Note that a small percentage of the children (17\%) had a 
normal weight at baseline. These children were overweight or obese at the moment they were included in the study, but turned to the upper range of a normal weight status score. We decided not to exclude these children from the study as prevention of excessive weight gain is also highly relevant for these children (i.e. not returning to the overweight status), and every child may benefit from an intervention aimed at improving parenting, diet and physical activity levels.

\section{Study sample}

Regarding the representativeness of the study sample for the Dutch population (CBS, 2005), it should be mentioned that our study sample was relatively highly educated. Lower attendance rates by lower socioeconomic groups is a well-known problem in childhood obesity intervention studies (Summerbell et al., 2005). Furthermore, a relatively high percentage of our parents were overweight or obese, viz. $80 \%$ of the fathers and $65 \%$ of the mothers of our sample, compared to $54 \%$ of men and $43 \%$ of women in the general population (CBS, 2012). However, this may be inherent to the problem of the children being overweight. The nationality of the parents who participated in the study was mainly Dutch, while the prevalence of the overweight is known to be higher among Moroccan and Turkish families in the Netherlands. However these families are mainly living in densely populated urban areas, which were not part of our research area.

An inherent aspect of study samples in randomized controlled trials is that they often include highly motivated participants. This may have been a problem in the current RCT as well, although $30 \%(N=13)$ of the families who were randomized to the intervention condition did not attend the group sessions. Reasons mentioned by parents for not attending the group sessions included lack of time and no babysitter, which is in line with the findings of similar studies (Jago et al., 2012). However, these reasons may also indicate an underlying problem, i.e. being insufficiently motivated.

The drop-out rate was $17 \%$ at 4 months (i.e. at the end of the intervention) and $22 \%$ at 12 months (i.e. at the final follow-up assessment). These attrition rates are relatively low compared to those found in other pediatric weight management programs, in which attrition was reported to be between 27 and 73\% (Skelton \& Beech, 2011). Attrition percentages were more or less comparable in the intervention and control conditions ( $18 \%$ and $17 \%$ at 4 months, $20 \%$ and $21 \%$ at 12 months), indicating the absence of attrition bias.

\section{Considerations regarding the effects of the intervention}

No intervention effects were found on children's body composition. Analyses of secondary outcome measures showed positive intervention effects on child behavior, 
parenting practices, parenting styles, parental feeding styles, and self-efficacy in parenting. Different explanations for and/or considerations about the apparent lack of effects on primary outcome measures are outlined below. The considerations concern the context of general parenting, intervention content, implementation, outcome measures, and statistical analyses.

\section{General parenting in perspective}

The Lifestyle Triple P intervention aims to change parenting behavior in order to influence child behavior, and finally to affect children's weight-related outcomes. This causal chain is rather long, and possibly explains why we found effects of the intervention on behavior, but not (yet) on the children's body composition. It is possible that behavioral changes which are sustained over longer time (> 12 months) may lead to small but persistent changes in children's weight development.

Furthermore, parenting does not occur in isolation, but in a particular context. In addition to the home environment, children are also exposed to other environments, for example school, out-of-school care, the neighborhood, and the media. An intervention which focuses only on parts of a complex system may be unlikely to produce large effects.

\section{Intervention content}

Overall, the parents considered the strategies in the intervention to be useful, although some intervention strategies were appreciated more than others. For example, parents found the 'reading food labels' strategy quite instructive. Strategies for managing misbehavior were considered by parents as another useful part of the program. However, the session in which the strategies for misbehavior were discussed was perceived as overloaded. In another session, parents were challenged to participate in active games, and the majority of the parents liked this session very much. The discussion of a broad range of strategies in the group sessions is an inherent part of Lifestyle Triple $\mathrm{P}$, and parents have to choose which strategy they consider useful in their personal situation. A drawback of the broad range of strategies may be that this might be overwhelming for parents.

The nutrition guidelines that were included in the Lifestyle Triple $P$ intervention were not always exactly in line with the guidelines of the Dutch Nutrition Centre, and this was sometimes confusing for the parents. Therefore, it was decided to adjust these components to the Dutch situation. Parents received separate worksheets containing the Dutch guidelines.

In addition to a workbook, parents received a booklet with ideas for active play and a recipe booklet. Parents indicated that the active games booklet was useful, but that the recipes were too difficult. In addition, they indicated that they would have liked a 'booster' session, to refresh their skills and knowledge. 


\section{Intervention implementation}

Implementing a new intervention in a specific country may be associated with specific challenges. The Lifestyle Triple P intervention materials were translated into Dutch for the current RCT. The manual for practitioners was not translated, which the Dutch practitioners perceived as a barrier to delivering the sessions. Practitioners had to be trained to use the new intervention materials. A specialized Lifestyle Triple P trainer flew over from the UK and delivered the training for the Dutch practitioners in English, which may have led to language obstacles.

Some of the intervention components were pilot-tested, but there were neither the resources nor the time to test the full program in one or more groups of parents before the RCT. As a result, the first groups who were trained were part of our RCT. Practitioners may have needed some practice before they felt that they master the intervention.

The intervention was delivered by three different professionals, who delivered it in pairs as part of the quality assurance effort. Although systematic formative and informative evaluation took place on a regular basis, no other practitioners were delivering the Lifestyle Triple $P$ intervention in the Netherlands at the time of the RCT, limiting the opportunities for exchange of knowledge and experiences.

Overall, the majority of the sessions (58 of the 60 sessions in 6 groups) were implemented as intended. Thirty percent $(N=13)$ of the families who were assigned to the intervention condition did not attend any of the group sessions. Of the parents who attended at least one group session, $81 \%(N=25)$ were present at 5 or more sessions. These parents were similar in terms of demographic characteristics to the parents who did not attend the group sessions. Secondary analyses, excluding parents who did not attend any group session, yielded similar results on the primary outcome measures as the results presented in chapter 8 .

Parents who were attending the group sessions generally had 2-3 telephone sessions. Reasons for not having telephone sessions were: holidays, difficulty to reach parents by telephone, and lack of time. Parents rated the value of intervention as 7.6 on a 10 -point scale $(84 \% \leq 7)$.

The developers of Triple $P$ envisioned it as a multilevel system intervention, incorporating 5 levels of intervention on a tiered continuum with increasing strength (Sanders, 2012). The rationale behind this strategy is that different parents and children have different needs. Implementing all 5 levels of Triple P simultaneously has been found to lead to population-level change (Sanders, 2012). An advantage of implementing a parenting intervention at various levels is that seeking parenting support is destigmatized. In the current study, the Lifestyle Triple P intervention was implemented in isolation and this suboptimal context may have decreased the potential of the intervention to produce effects on primary outcomes. 


\section{Outcome measures}

Children's body composition was objectively measured using anthropometry, by a research assistant who was blinded to the research condition. Weight and height were measured to determine children's standardized BMI scores. Standardized BMI values (BMI z-scores) were used based on a Dutch reference population, i.e., the Fourth Dutch National Growth Study (Fredriks et al., 2000). Generally, a BMI above the $85^{\text {th }}$ percentile is defined as overweight, and a $\mathrm{BMI}$ above the $95^{\text {th }}$ percentile as obesity (Barlow, 2007). A shortcoming of using BMI and BMI z-scores is that is this measure does not discriminate between fat mass and fat-free mass. Whereas children who are physically inactive may increase their BMI by increasing fat mass, children who are more physically active may increase their BMI due to increased muscle mass. We attempted to overcome this problem by including additional measures to indicate body composition, i.e. waist circumference and biceps and triceps skinfolds. Both measures are simple, inexpensive, and quick, although both have limited reliability (Waters et al., 2011).

Physical activity was measured using Actigraph accelerometers (GT1M), an objective method which provides quantitative information about the vertical acceleration of the trunk (Trost, 2007). A disadvantage of accelerometers is their insensitivity to some horizontal movements of the body, leading to underestimation of children's physical activity levels. Furthermore, compliance with wearing the accelerometers may be low, especially among overweight children, because it may lead to stigmatizing and an increased risk of bullying (Robertson, Stewart-Brown, Wilcock, Oldfield, \& Thorogood, 2011). In our study, low compliance was not a major problem: only a small minority (about 10\%) of the children was unwilling to wear the accelerometers.

Accelerometers are widely accepted as a valid way to measure young children's physical activity level (Trost, 2007). Because accelerometers do not provide information about the type of physical activity, however, items on specific types of physical activity were included in the questionnaire for parents. These questions were based on a standard questionnaire used in Dutch youth health care, which was also used in earlier research (Gubbels et al., 2011a). It should be noted that questionnaire data may yield higher levels of physical activity than to accelerometer data (Schokker, Hekkert, Kocken, Van de Brink, \& De Vries, 2012).

Self-report questionnaires were filled out by one of the parents. Questionnaires are prone to information bias, for example due to social desirability. However, in selecting the questionnaires, we tried to include validated questionnaires as much as possible. Particularly, we used items from the Child Rearing Practices Report (Dekovic, 1989) and validated psychological control items (Barber, 1996) to measure general parenting, as well as parenting practices items from the Child Feeding Questionnaire (Birch et al., 2001), feeding styles items from the validated Children's Eating Behavior 
Questionnaire (Wardle et al., 2001; Sleddens, Kremers, De Vries, \& Thijs, 2010), and the validated Parenting Satisfaction and Efficacy Scale to measure parental effectiveness and satisfaction (Johnston \& Mash, 1989). In addition, children's dietary intake and behavior were assessed using items from existing questionnaires (Gubbels et al., 2009a; Gubbels et al., 2009b).

\section{Statistical analyses}

Intention-to-treat analyses (ITT) are considered the standard approach for determining whether an intervention is effective. However, this kind of analysis is only adequate when the amount of missing data is minimal (Hollis \& Campbell, 1999). Complete-case analyses can be problematic if part of the data is lacking. In that case it is important to consider whether the missing data is random. This is often not the case, as subjects who drop-out often do worse than those who continue the intervention. In the current $\mathrm{RCT}$, drop-out rates were similar in the intervention and control conditions.

In the case of missing data, there are several strategies available to treat the data, and using multiple imputation models to impute the data is currently considered a good approach (Van Buuren, 2012). However, in the present study, the application of multiple imputation yielded smaller effects. The best treatment of missing values remains subject to debate (Bodner, 2008; Graham, Olchowski, \& Gilreath, 2007; Schulz et al., 2013; White, Royston, \& Wood, 2011).

Multi-level analyses had to be performed to account for possible differential effects in different study groups. The use of multi-level analysis of multiple imputation data is still under-investigated, and more research is necessary in this area in order to provide reliable estimates (Van Buuren, 2012).

\section{Implications for practice}

The findings from the studies discussed in the current thesis have several implications for practice. These implications relate to the recruitment of participants for obesity prevention interventions, prevention and treatment of childhood obesity, the content of the intervention, and the implementation of the Lifestyle Triple P intervention.

\section{Recruitment of participants}

Active recruiting of parents of overweight children was found to be the most effective strategy in the current study. However, attention should be paid to the way parents are approached. Health care professionals who are referring parents and children to obesity interventions may not explicitly focus on the children's weight status. Parents may feel that the weight status of their child is a result of a complex interaction of 
behaviors and thus outside their control, resulting in denial and resistance. Furthermore, parents may not immediately perceive it as a problem that their child is somewhat heavier. As an alternative, we recommend that health care professionals take parenting or the general health of children as the basis for the conversation with parents. These concepts are directly related to the children's weight status, but may feel less confrontational to parents.

Another option is to use the Lifestyle Behavior Checklist as a basis for recruitment. This instrument measures parental perception of problems related to overweight and their confidence in managing these problems. As was shown in chapter 5 , parents of overweight children experience more weight-related problems and have lower selfefficacy in managing these problems. These problems are relevant to parents.

In order to ensure that YHC professionals have sufficient skills and self-efficacy to cope with parental resistance, they could be trained in communication skills, such as Motivational Interviewing (Miller \& Rollnick, 2002).

\section{Prevention and treatment of childhood obesity}

Health professionals working with overweight or obese children should involve parents in their efforts. However, they should realize that these parents often underestimate their child's overweight, and our findings show that being aware of one's child's overweight as such does not seem sufficient for subsequent weight management (see chapter 4). It is important that parents become aware that this overweight should be managed in a healthy and sustainable fashion (i.e. not by putting their child on a strict diet). Health professionals should guide parents in ways to improve health habits by focusing on improving both parenting practices and parenting styles. In this respect, parents should become aware of their influence on their children's health behaviors, for instance by reflecting on these health behaviors and related parenting behaviors. Health professionals should help parents choose realistic goals relating to these behaviors and offer effective strategies for reaching these goals (for example using active transport, using water as the main drink or setting a good example as a parent). Health behavior changes should be embedded in a positive parenting context, in which parents praise desired child behaviors, and are involved, strict and consistent in relation to their child.

\section{Lifestyle Triple P intervention}

Several recommendations can be made for changes to the content of the intervention. With regard to the nutrition strategies, we recommend including Dutch national nutrition guidelines from the Nutrition Centre. Moreover, the recipes from the recipe booklet should be simplified, as they were perceived as too difficult and not always adapted 
to Dutch eating habits. Furthermore, a 'booster' session should be included to refresh parental skills and knowledge, for example about 6 months after the final group session.

\section{Implementation of Lifestyle Triple P}

Based on the results of the current study, wider implementation of the Lifestyle Triple $P$ intervention cannot be recommended at this point in time. The intervention should be adjusted as described in the above paragraph, after which further evaluation studies should be conducted to assess the effects of the adjusted intervention. However, it is an interesting exercise to think somewhat further ahead and consider where the Lifestyle Triple P intervention could be implemented in the current health care system. In the Netherlands, the integrated approach to overweight and obesity distinguishes three levels of obesity prevention: (1) prevention programs for the healthy population, (2) early detection of overweight children and adolescents using the signaling protocol (these children are exposed to the 'prevention protocol'), and (3) guidelines for treating obesity (Seidell, De Beer, \& Kuijpers, 2008). In practice, this means that parents of overweight children receive the overweight 'prevention protocol' (Be Active Eat Right study) (Veldhuis et al., 2009b), which consists of a maximum of three structured healthy lifestyle counseling sessions with parents. Obese children are referred to a pediatrician for intensive treatment.

One aspect to consider here is whether the Lifestyle Triple P intervention is part of the prevention or treatment of childhood obesity. In the current study, the intervention was used to prevent further excessive weight gain by overweight children, whereas the Australian trial performed by West and colleagues recruited mainly obese children, which suggests treatment.

If the Lifestyle Triple P intervention should prove to be effective, the intervention should be offered as a prevention program to parents of overweight children who need support in parenting. Furthermore, parents of obese children who receive treatment in hospital could also be given the option of receiving the Lifestyle Triple P intervention, complementary to their child's hospital treatment.

Optimal implementation of the Lifestyle Triple $P$ intervention will be achieved when different levels of the Triple $P$ intervention are implemented simultaneously. In particular, a Lifestyle Triple $P$ intervention at level 2 (three seminars in public places such as schools, on positive parenting, diet, and physical activity) has been developed and is currently being evaluated. Earlier studies showed that Triple P seminars increased parenting skills and reduced childhood problems (Sanders, Prior, \& Ralph, 2009). These seminars might also foster increased awareness among the population and recruitment of parents for the Lifestyle Triple $P$ group sessions. 


\section{Implications for further research}

In addition to recommendations for practice, some implications for theory and further research are outlined below.

\section{Measurement instruments}

An important part of intervention research depends on the use of validated and reliable evaluation instruments. In particular, further research should be done into the validity of the Dutch translation of the Lifestyle Behavior Checklist (LBC). The LBC measures children's problem behavior and parental self-efficacy in managing this problem behavior. The study reported on in chapter 5 tested the convergent validity of the LBC (the degree to which two methods yield similar results); future validation studies should also test the discriminant validity (the degree to which a single method of measuring two distinct constructs leads to different results) in order to fully assess the measure's construct validity (Polit \& Beck, 2008). For example, West and colleagues tested the scale's discriminant validity by correlating the LBC with the Strengths and Difficulties Questionnaire (SDQ; Goodman, 1997).

Self-efficacy is a strong predictor of a person's behavior (Bandura, 1977), and it is particularly in the general parenting literature that self-efficacy is considered an important determinant of parenting behaviors (Coleman \& Karraker, 2000; Jones \& Prinz, 2005). Unfortunately, the construct of weight-related parenting self-efficacy has so far been neglected in research into food and activity parenting (Nelson \& Davis, 2013; Smith et al., 2010). More research on the effect of parenting self-efficacy in managing children's energy-balance related behaviors would therefore be valuable.

\section{Prevention of childhood obesity}

When developing and evaluating intervention programs for childhood obesity, it is important to consider the broader ecological context. In addition to the home environment, children are also exposed to the school environment, day care centers, and the broader community. All these environments can be considered as potentially influencing children's behaviors and weight status, and are therefore potential gateways for interventions. Additionally, local governments, non-governmental organizations and the media could be involved (Summerbell et al., 2012). Ideally, the same goal, i.e. prevention of childhood obesity, should be strived for simultaneously in all different environments to achieve optimal effects.

In addition to distinguishing various relevant micro- and macro-environments, intervention developers should also consider different types of environment. Swinburn (1999) identified four different types of environment: physical (what is available), sociocultural (social and cultural values), economic (costs related to food and physical 
activity) and political (laws, regulations, policies). All these types of environment contribute to overweight development. The Lifestyle Triple P intervention mainly focuses on changing the child's immediate social environment, i.e. the parents, although some factors within the physical environment (e.g., availability of healthy food) and the political environment (e.g., rules at home) are also part of the intervention. It would be an important step forward if future intervention developers were to consider all four types of environments to a fuller extent.

General parenting is considered an important influencing factor explaining children's weight-related health behaviors, see chapter 2. General parenting is a general concept determining the context of behavior-specific parenting. A positive change in this concept may therefore impact on a broad range of specific parenting practices, resulting in a broad public health effect. Nevertheless, at the time when we conducted our systematic review on parenting for the prevention or treatment of childhood obesity (see chapter 3), only 7 intervention studies could be found. Recently, several new studies on general parenting interventions to prevent childhood obesity have been published (Brotman et al., 2012; Magarey et al., 2011; Moens \& Braet, 2012; Ostbye et al., 2012; Willis et al., 2013). A recent review indicated that the family can play an import role in modifying the lifestyle of overweight children (Sung-Chan, Sung, Zhao, \& Brownson, 2013). However, research in this field is still ongoing and more research on general parenting interventions would be valuable.

\section{Further research into Lifestyle Triple P}

Future research should investigate the effectiveness of the Lifestyle Triple P intervention at longer-term follow-up (> 1 year), to assess whether the behavioral changes are sustained and whether these changes have an effect on anthropometric outcomes in the long run. Further research should also be done into the effectiveness of the Lifestyle Triple $\mathrm{P}$ intervention in different settings, in different parts of the Netherlands, and in larger and different samples (for example lower socioeconomic groups and ethnic minorities).

Additionally, further research in larger samples should investigate the working mechanisms of the intervention, using mediation and moderation analyses. An essential component of the Triple $P$ intervention is stimulating parental self-regulation (Sanders \& Mazzucchelli, 2013). Self-regulation is the process wherein individuals acquire the skills they need to change their own behavior and become independent problem solvers. In order to examine whether behavior change occurs via parental self-regulation, a validated questionnaire measuring self-regulation should be included in future studies (Sanders \& Mazzucchelli, 2013).

Another important part of evaluation research is the cost-effectiveness of an intervention. Several studies on the cost-effectiveness of the standard Triple P inter- 
vention have already been conducted, and these studies provided evidence that the intervention is cost-effective (Aos et al., 2011; Foster, Prinz, Sanders, \& Shapiro, 2008; Mihalopoulos, Sanders, Turner, Murphy-Brennan, \& Carter, 2007; Mihalopoulos, Vos, Pirkis, \& Carter, 2011). However, no cost-effectiveness studies have been conducted so far for the Lifestyle Triple P intervention. If Lifestyle Triple P should prove effective, future studies should consider its cost-effectiveness as well.

\section{General conclusions}

General parenting is an important determinant in explaining children's weight-related outcomes. Lifestyle Triple $\mathrm{P}$ is a general parenting intervention which aims to prevent further weight gain in overweight and obese children. The current thesis reports on an RCT investigating the effect of Lifestyle Triple P on children aged 4 to 8 years in the Netherlands. Although the intervention showed positive effects on various child behaviors and parenting measures, no effects were found on children's body composition, 4 and 12 months after baseline. 


\section{References}

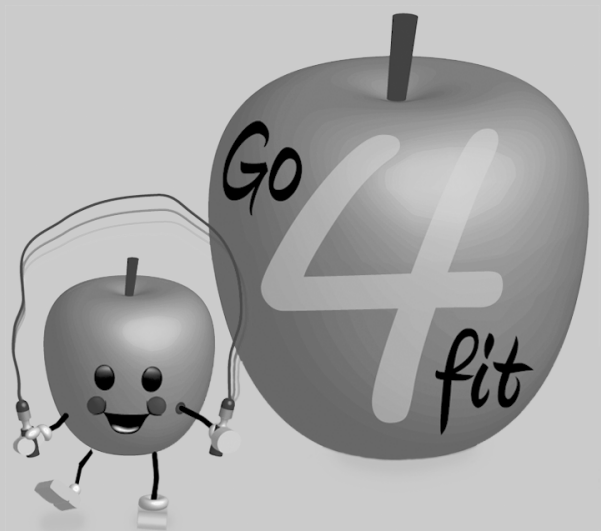




\section{4 | REFERENCES}

Agras, W. S., Hammer, L. D., McNicholas, F., \& Kraemer, H. C. (2004). Risk factors for childhood overweight: a prospective study from birth to 9.5 years. Journal of Pediatrics, 145(1), 20-25.

Aos, S., Lee, S., Drake, E., Pennucci, A., Klima, T., Miller, M., Anderson, L., Mayfield, J., \& Burley, M. (2011). Return on investment: Evidence-based options to improve statewide outcomes. Olympia: Washington State Institute for Public Policy.

Aragona, J., Cassady, J., \& Drabman, R. S. (1975). Treating overweight children through parental training and contingency contracting. Journal of Applied Behavior Analysis, 8(3), 269-278.

Arnold, D. S., O'Leary, S. G., Wolff, L. S., \& Acker, M. M. (1993). The Parenting Scale: A measure of dysfunctional parenting in discipline situations. Psychological Assessment, 5(2), 137.

Baldwin, A. L. (1948). Socialization and the parent-child relationship. Child Development, 19(3), 127-136.

Bandura, A. (1971). Social learning theory of aggression. In J. F. Knutson (Ed.), Control of aggression: implications from basic research (pp. 201-250). Chicago: Adline Publishing Co.

Bandura, A. (1977). Self-efficacy: toward a unifying theory of behavioural change. Psychological Review, $84(2), 191-215$.

Bandura, A. (1995). Self-efficacy in changing societies: Cambridge University Press.

Barber, B. K. (1996). Parental psychological control: Revisiting a neglected construct. Child Development, 67(6), 3296-3319.

Barber, B. K., \& Harmon, E. L. (2002). Violating the self: Parental psychological control of children and adolescents Intrusive parenting: how psychological control affects children and adolescents. (pp. 1552). Washington DC: American Psychological Press.

Barkham, M., \& Mellor-Clark, J. (2003). Bridging evidence based practice and practice based evidence: developing a rigorous and relevant knowledge for the psychological therapies. Clinical Psychology \& Psychotherapy, 10(6), 319-327.

Barlow, J., \& Stewart-Brown, S. (2006). Understanding parenting programmes: parents' views. Primary Health Care Research and Development, 2(02), 117-130.

Barlow, S. E. (2007). Expert committee recommendations regarding the prevention, assessment, and treatment of child and adolescent overweight and obesity: summary report. Pediatrics, 120 Suppl 4, S164-192.

Baughcum, A. E., Chamberlin, L. A., Deeks, C. M., Powers, S. W., \& Whitaker, R. C. (2000). Maternal perceptions of overweight preschool children. Pediatrics, 106(6), 1380-1386.

Baumrind, D. (1965). Parental control and parental love. Children, 12(6), 230-234.

Baumrind, D. (1967). Child care practices anteceding three patterns of preschool behavior. Genetic Psychology Monographs, 75(1), 43-88.

Baumrind, D. (1978). Parental disciplinary patterns and social competence in children. Youth \& Society, 9(3), 239-276.

Beck, S., \& Terry, K. (1985). A comparison of obese and normal-weight families' sychological characteristics. The American Journal of Family Therapy, 13(3), 55-59.

Becker, W. C. (1964). Consequences of different kinds of parental discipline. Review of child development research, 1, 169-208.

Berge, J. M., Wall, M., Bauer, K. W., \& Neumark-Sztainer, D. (2010a). Parenting characteristics in the home environment and adolescent overweight: a latent class analysis. Obesity, 18(4), 818-825.

Berge, J. M., Wall, M., Loth, K., \& Neumark-Sztainer, D. (2010b). Parenting style as a predictor of adolescent weight and weight-related behaviors. Journal of Adolescent Health, 46(4), 331-338.

Biglan, A. (1995). Translating what we know about the context of antisocial behavior into a lower prevalence of such behavior. Journal of Applied Behavior Analysis, 28(4), 479-492.

Birch, L. L., Fisher, J. O., Grimm-Thomas, K., Markey, C. N., Sawyer, R., \& Johnson, S. L. (2001). Confirmatory factor analysis of the Child Feeding Questionnaire: a measure of parental attitudes, beliefs and practices about child feeding and obesity proneness. Appetite, 36(3), 201-210. 
Blissett, J., \& Haycraft, E. (2008). Are parenting style and controlling feeding practices related? Appetite, 50(2-3), 477-485.

Block, J. H. (1965). The child-rearing practices report. Berkeley, CA: Institute of Human Development, University of California, 548-581.

Block, J. H. (1981). The Child-Rearing Practices Report (CRPR): a set of Q items for the description of parental socialization attitudes and values. Institute of Human Development, University of California, Berkely. Unpublished manuscript.

Bluford, D. A. A., Sherry, B., \& Scanlon, K. S. (2007). Interventions to prevent or treat obesity in preschool children: a review of evaluated programs. Obesity, 15(6), 1356-1372.

Bodner, T. E. (2008). What improves with increased missing data imputations? Structural Equation Modeling, 15(4), 651-675.

Bolling, C., Crosby, L., Boles, R., \& Stark, L. (2009). How pediatricians can improve diet and activity for overweight preschoolers: a qualitative study of parental attitudes. Acta Paediatrica, 9(3), 172-178.

Brann, L. S., \& Skinner, J. D. (2005). More controlling child-feeding practices are found among parents of boys with an average body mass index compared with parents of boys with a high body mass index. Journal of the American Dietetic Association, 105(9), 1411-1416.

Brotman, L. M., Dawson-McClure, S., Huang, K. Y., Theise, R., Kamboukos, D., Wang, J., Petkova, E., \& Ogedegbe, G. (2012). Early childhood family intervention and long-term obesity prevention among high-risk minority youth. Pediatrics, 129(3), e621-628.

Brown, K. A., Ogden, J., Vogele, C., \& Gibson, E. L. (2008). The role of parental control practices in explaining children's diet and BMI. Appetite, 50(2-3), 252-259.

Bulk-Bunschoten, A., Renders, C., Leerdam, F. J. M., \& Hirasing, R. (2004, Mei 2004). Signaleringsprotocol overgewicht in de jeugdgezondheidszorg. VUmc Amsterdam.

Buri, J. R. (1991). Parental authority questionnaire. Journal of Personality Assessment, 57(1), 110-119.

Campbell, K. J., \& Hesketh, K. D. (2007). Strategies which aim to positively impact on weight, physical activity, diet and sedentary behaviours in children from zero to five years. A systematic review of the literature. Obesity Reviews, 8(4), 327-338.

Campbell, M., Fitzpatrick, R., Haines, A., Kinmonth, A. L., Sandercock, P., Spiegelhalter, D., \& Tyrer, P. (2000). Framework for design and evaluation of complex interventions to improve health. British Medical Journal, 321(7262), 694-696.

Centraal Bureau voor de Statistiek. (2005). Nederlandse bevolking steeds hoger opgeleid. Webmagazine, 25 juli 2005

Centraal Bureau voor de Statistiek. (2012). Nederland steeds langer maar vooral zwaarder. Webmagazine, 3 december 2012

Chaparro, M. P., Langellier, B. A., Kim, L. P., \& Whaley, S. E. (2011). Predictors of accurate maternal perception of their preschool child's weight status among Hispanic WIC participants. Obesity, 19(10), 2026-2030.

Chen, J. L., \& Kennedy, C. (2004). Family functioning, parenting style, and Chinese children's weight status. Journal of Family Nursing, 10(2), 262-279.

Chen, J. L., \& Kennedy, C. (2005). Factors associated with obesity in Chinese-American children. Pediatric Nursing, 31(2), 110-115.

Chen, J. L., Kennedy, C., Yeh, C. H., \& Kools, S. (2005). Risk factors for childhood obesity in elementary school-age taiwanese children. Progress in Cardiovascular Nursing, 20(3), 96-103.

Chen, J. L., Unnithan, V., Kennedy, C., \& Yeh, C. H. (2008). Correlates of physical fitness and activity in Taiwanese children. International Nursing Review, 55(1), 81-88.

Choi, L., Liu, Z., Matthews, C. E., \& Buchowski, M. S. (2011). Validation of accelerometer wear and nonwear time classification algorithm. Medicine \& Science in Sports \& Exercise, 43(2), 357-364.

Cohen, J. (1988). Statistical power analysis for the behavioral sciences: Lawrence Erlbaum.

Cohen, J. (1992). A power primer. Psychological Bulletin, 112(1), 155-159. 


\section{REFERENCES}

Cole, T. J., Bellizzi, M. C., Flegal, K. M., \& Dietz, W. H. (2000). Establishing a standard definition for child overweight and obesity worldwide: international survey. British Medical Journal, 320, 1240-1243.

Coleman, P. K., \& Karraker, K. H. (2000). Parenting self-efficacy among mothers of school-age children: conceptualization, measurement, and correlates. Family Relations, 49(1), 13-24.

Croake, J., \& Hinkle, D. (1991). Attitudes toward child rearing scale (ATCRS) The ETS Test Collection Catalog. Phoenix, AZ: Oryx Press.

Crosby, R. A., DiClemente, R. J., \& Salazar, L. F. (2006). Research methods in health promotion: LibreDigital.

Daniels, L. A., Magarey, A., Battistutta, D., Nicholson, J. M., Farrell, A., Davidson, G., \& Cleghorn, G. (2009). The NOURISH randomised control trial: positive feeding practices and food preferences in early childhood - a primary prevention program for childhood obesity. BMC Public Health, 9, 387.

Daniels, S. (2009). Complications of obesity in children and adolescents. International Journal of Obesity, 33, S60-S65.

Darling, N., \& Steinberg, L. (1993). Parenting style as context: an integrative model. Psychological Bulletin, 113, 487-496.

Davison, K. K., Cutting, T. M., \& Birch, L. L. (2003). Parents' activity-related parenting practices predict girls' physical activity. Medicine and Science in Sports and Exercise, 35(9), 1589-1595.

De Bourdeaudhuij, I., Te Velde, S. J., Maes, L., Perez-Rodrigo, C., De Almeida, M. D., \& Brug, J. (2009). General parenting styles are not strongly associated with fruit and vegetable intake and socialenvironmental correlates among 11-year-old children in four countries in Europe. Public Health Nutrition, 12(2), 259-266.

De Graaf, I., Speetjens, P., Smit, F., De Wolff, M., \& Tavecchio, L. (2008a). Effectiveness of the Triple P Positive Parenting Program on behavioral problems in children: a meta-analysis. Behavior Modification, 32(5), 714-735.

De Graaf, I., Speetjens, P., Smit, F., De Wolff, M., \& Tavecchio, L. (2008b). Effectiveness of the Triple P Positive Parenting Program on parenting: a meta-analysis. Family Relations, 57(5), 553-566.

De Hoog, M. L., Stronks, K., Van Eijsden, M., Gemke, R. J., \& Vrijkotte, T. G. (2012). Ethnic differences in maternal underestimation of offspring's weight: the ABCD study. International Journal of Obesity, 36(1), 53-60.

De La, O. (2009). Do parents accurately perceive their child's weight status? Journal of Pediatric Health Care, 23(4), 216-221.

De Onis, M., Blössner, M., \& Borghi, E. (2010). Global prevalence and trends of overweight and obesity among preschool children. The American journal of clinical nutrition, 92(5), 1257-1264.

Dekovic, M. (1989). Measuring dimensions and patterns of child rearing: Dutch version of the block child rearing practices report (CRPR). Internal publication, University of Nijmegen, The Netherlands.

Dekovic, M., Janssens, J. M., \& Gerris, J. R. (1991). Factor structure and construct validity of the Block Child Rearing Practices Report (CRPR). Psychological Assessment: A Journal of Consulting and Clinical Psychology, 3(2), 182.

Den Exter Blokland, E. A. W., Engels, R. C. M. E., \& Finkenauer, C. (2001). Parenting-styles, self-control and male juvenile delinquency Prevention and control of aggression and the impact on its victims (pp. 201207). Dordrecht/New York: Kulwer/Plenum Press.

Dhingra, A., Brennan, L., \& Walkley, J. (2011). Predicting treatment initiation in a family-based adolescent overweight and obesity intervention. Obesity, 19(6), 1307-1310.

Doak, C. M., Visscher, T. L., Renders, C. M., \& Seidell, J. C. (2006). The prevention of overweight and obesity in children and adolescents: a review of interventions and programmes. Obesity Reviews, 7(1), 111136.

Du, H., \& Feskens, E. (2010). Dietary determinants of obesity. Acta cardiologica, 65(4), 377-386.

Dusenbury, L., Brannigan, R., Falco, M., \& Hansen, W. B. (2003). A review of research on fidelity of implementation: implications for drug abuse prevention in school settings. Health Education Research, 18(2), 237-256. 
Eckstein, K. C., Mikhail, L. M., Ariza, A. J., Thomson, J. S., Millard, S. C., \& Binns, H. J. (2006). Parents' perceptions of their child's weight and health. Pediatrics, 117(3), 681-690.

Eijkemans, M., Mommers, M., De Vries, S. I., van Buuren, S., Stafleu, A., Bakker, I., \& Thijs, C. (2008). Asthmatic symptoms, physical activity, and overweight in young children: a cohort study. Pediatrics, 121(3), e666-672.

Emery, R. E. (1982). Interparental conflict and the children of discord and divorce. Psychological Bulletin, 92(2), 310-330.

Enten, R. S., \& Golan, M. (2008). Parenting styles and weight-related symptoms and behaviors with recommendations for practice. Nutrition Reviews, 66(2), 65-75.

Epstein, L. H., Gordy, C. C., Raynor, H. A., Beddome, M., Kilanowski, C. K., \& Paluch, R. (2001a). Increasing fruit and vegetable intake and decreasing fat and sugar intake in families at risk for childhood obesity. Obesity Research, 9(3), 171-178.

Epstein, L. H., McCurley, J., Wing, R. R., \& Valoski, A. (1990). Five-year follow-up of family-based behavioral treatments for childhood obesity. Journal of Consulting and Clinical Psychology, 58(5), 661-664.

Epstein, L. H., McKenzie, S. J., Valoski, A., Klein, K. R., \& Wing, R. R. (1994a). Effects of mastery criteria and contingent reinforcement for family-based child weight control. Addictive Behaviors, 19(2), 135-145.

Epstein, L. H., Paluch, R. A., \& Raynor, H. A. (2001b). Sex differences in obese children and siblings in familybased obesity treatment. Obesity Research, 9(12), 746-753.

Epstein, L. H., Valoski, A., Wing, R. R., \& McCurley, J. (1990). Ten-year follow-up of behavioral, family-based treatment for obese children. Journal of the American Association, 264(19), 2519-2523.

Epstein, L. H., Valoski, A., Wing, R. R., \& McCurley, J. (1994b). Ten-year outcomes of behavioral family-based treatment for childhood obesity. Health Psychology, 13(5), 373-383.

Epstein, L. H., Wing, R. R., Koeske, R., \& Valoski, A. (1985). A comparison of lifestyle exercise, aerobic exercise, and calisthenics on weight loss in obese children. Behavior Therapy, 16(4), 345-356.

Eurostat. (2007). Taksforce on Core Social Variables. Final Report. Luxembourg: European Press Office.

Evenson, K. R., Catellier, D. J., Gill, K., Ondrak, K. S., \& McMurray, R. G. (2008). Calibration of two objective measures of physical activity for children. Journal of Sports Science, 26(14), 1557-1565.

Faith, M. S., Scanlon, K. S., Birch, L. L., Francis, L. A., \& Sherry, B. (2004). Parent-child feeding strategies and their relationships to child eating and weight status. Obesity Research, 12(11), 1711-1722.

Finne, E., Reinehr, T., Schaefer, A., Winkel, K., \& Kolip, P. (2009). Overweight children and adolescents-is there a subjective need for treatment? International Journal of Public Health, 54(2), 112-116.

Fletcher, R., Freeman, E., \& Matthey, S. (2011). The impact of behavioural parent training on fathers' parenting: a meta-analysis of the Triple P-Positive Parenting Program. Fathering: A Journal of Theory, Research, and Practice about Men as Fathers, 9(3), 291-312.

Fleuren, M., Wiefferink, K., \& Paulussen, T. (2004). Determinants of innovation within health care organizations: literature review and Delphi study. International Journal of Quality in Health Care, 16(2), 107-123.

Flodmark, C. E., Ohlsson, T., Rydén, O., \& Sveger, T. (1993). Prevention of progression to severe obesity in a group of obese schoolchildren treated with family therapy. Pediatrics, 91(5), 880-884.

Foster, E. M., Prinz, R. J., Sanders, M. R., \& Shapiro, C. J. (2008). The costs of a public health infrastructure for delivering parenting and family support. Children and Youth Services Review, 30(5), 493-501.

Foster, N., \& Little, P. (2012). Methodological issues in pragmatic trials of complex interventions in primary care. British Journal of General Practice, 62, 10-11.

Fredriks, A. M., Van Buuren, S., Wit, J. M., \& Verloove Vanhorick, S. P. (2000). Body index measurements in 1996-7 compared with 1980. Archives of Disease in Childhood, 82(2), 107-112.

Freedman, D. S., Khan, L. K., Serdula, M. K., Dietz, W. H., Srinivasan, S. R., \& Berenson, G. S. (2005). The relation of childhood BMI to adult adiposity: The Bogalusa Heart Study. Pediatrics, 115(1), 22.

Gable, S., \& Lutz, S. (2000). Household, parent, and child contributions to childhood obesity. Family Relations, 49(3), 293-300. 


\section{REFERENCES}

Garrett-Wright, D. (2011). Parental perception of preschool child body weight. Journal of Pediatric Nursing, 26(5), 435-445.

Gerards, S. M., Dagnelie, P. C., Jansen, M. W., De Vries, N. K., \& Kremers, S. P. (2012a). Barriers to successful recruitment of parents of overweight children for an obesity prevention intervention: a qualitative study among youth health care professionals. BMC Family Practice, 13(1), 37.

Gerards, S. M., Dagnelie, P. C., Jansen, M. W., Van der Goot, L. H., De Vries, N. K., Sanders, M. R., \& Kremers, S. P. (2012b). Lifestyle Triple P: a parenting intervention for childhood obesity. BMC Public Health, 12(1), 267.

Gerards, S. M., Sleddens, E. F., Dagnelie, P. C., De Vries, N. K., \& Kremers, S. P. (2011). Interventions addressing general parenting to prevent or treat childhood obesity. International Journal of Pediatric Obesity, 6(2-2), e28-45.

Gibson, L. Y., Byrne, S. M., Davis, E. A., Blair, E., Jacoby, P., \& Zubrick, S. R. (2007). The role of family and maternal factors in childhood obesity. Medical Journal of Australia, 186(11), 591-595.

Glasgow, R. E., Vogt, T. M., \& Boles, S. M. (1999). Evaluating the public health impact of health promotion interventions: the RE-AIM framework. American Journal of Public Health, 89(9), 1322-1327.

Golan, M. (2006). Parents as agents of change in childhood obesity-from research to practice. International Journal of Pediatric Obesity, 1(2), 66-76.

Golan, M., \& Crow, S. (2004). Parents are key players in the prevention and treatment of weight-related problems. Nutrition Reviews, 62(1), 39-50.

Golan, M., Fainaru, M., \& Weizman, A. (1998). Role of behaviour modification in the treatment of childhood obesity with the parents as the exclusive agents of change. International Journal of Obesity, 22(12), 1217-1224.

Golan, M., Kaufman, V., \& Shahar, D. R. (2006). Childhood obesity treatment: targeting parents exclusively v. parents and children. British Journal of Nutrition, 95(5), 1008-1015.

Golan, M., Weizman, A., Apter, A., \& Fainaru, M. (1998). Parents as the exclusive agents of change in the treatment of childhood obesity. American Journal of Clinical Nutrition, 67(6), 1130-1135.

Golley, R. K. (2005). Family-focused management of overweight in pre-pubertal children - a randomised controlled trial. The healthy eating and lifestyle through positive parenting (HELPP) study. (Doctor of Philosophy), School of Medicine, Flinders University.

Golley, R. K., Hendrie, G. A., Slater, A., \& Corsini, N. (2011). Interventions that involve parents to improve children's weight-related nutrition intake and activity patterns - what nutrition and activity targets and behaviour change techniques are associated with intervention effectiveness? Obesity Reviews, 12(2), 114-130.

Golley, R. K., Magarey, A. M., Baur, L. A., Steinbeck, K. S., \& Daniels, L. A. (2007a). Twelve-month effectiveness of a parent-led, family-focused weight-management program for prepubertal children: a randomized, controlled trial. Pediatrics, 119(3), 517-525.

Golley, R. K., Perry, R. A., Magarey, A., \& Daniels, L. (2007b). Family-focused weight management program for five-to nine-year-olds incorporating parenting skills training with healthy lifestyle information to support behaviour modification. Nutrition and Dietetics, 64(3), 144-150.

Goodman, R. (1997). The Strengths and Difficulties Questionnaire: a research note. The Journal of Child Psychology and Psychiatry, 38(5), 581-586.

Graham, J. W., Olchowski, A. E., \& Gilreath, T. D. (2007). How many imputations are really needed? Some practical clarifications of multiple imputation theory. Prevention Science, 8(3), 206-213.

Grolnick, W. S., \& Slowiaczek, M. L. (1994). Parents' involvement in children's schooling: a multidimensional conceptualization and motivational model. Child Development, 65(1), 237-252.

Grych, J. H., \& Fincham, F. D. (1990). Marital conflict and children's adjustment: A cognitive-contextual framework. Psychological Bulletin, 108(2), 267-290. 
Gubbels, J. S., Kremers, S. P., Stafleu, A., Dagnelie, P. C., De Vries, S. I., De Vries, N. K., \& Thijs, C. (2009a). Clustering of dietary intake and sedentary behavior in 2-year-old children. Journal of Pediatrics, 155(2), 194-198.

Gubbels, J. S., Kremers, S. P., Stafleu, A., Dagnelie, P. C., Goldbohm, R. A., De Vries, N. K., \& Thijs, C. (2009b). Diet-related restrictive parenting practices. Impact on dietary intake of 2-year-old children and interactions with child characteristics. Appetite, 52(2), 423-429.

Gubbels, J. S., Kremers, S. P., Stafleu, A., De Vries, S. I., Goldbohm, R. A., Dagnelie, P. C., De Vries, N. K., Van Buuren, S., \& Thijs, C. (2011a). Association between parenting practices and children's dietary intake, activity behavior and development of body mass index: the KOALA Birth Cohort Study. International Journal of Behavioural Nutrition and Physical Activity, 8, 18.

Gubbels, J. S., Thijs, C., Stafleu, A., Van Buuren, S., \& Kremers, S. P. (2011b). Association of breast-feeding and feeding on demand with child weight status up to 4 years. International Journal of Pediatric Obesity, 6(2-2), e515-522.

Hart, B., \& Risley, T. R. (1995). Meaningful differences in the everyday experience of young American children: Paul $\mathrm{H}$ Brookes Publishing.

Harvey-Berino, J., \& Rourke, J. (2003). Obesity prevention in preschool native-american children: a pilot study using home visiting. Obesity Research, 11(5), 606-611.

Haugstvedt, K. T., Graff-Iversen, S., Bechensteen, B., \& Hallberg, U. (2011). Parenting an overweight or obese child: a process of ambivalence. Journal of Child Health Care, 15(1), 71-80.

He, M., Irwin, J. D., Sangster Bouck, L. M., Tucker, P., \& Pollett, G. L. (2005). Screen-viewing behaviors among preschoolers parents' perceptions. American Journal of Preventive Medicine, 29(2), 120-125.

Hejazi, S. (2007). Temperament, parenting, and the development of childhood obesity. The University of British Columbia.

Hennessy, E., Hughes, S. O., Goldberg, J. P., Hyatt, R. R., \& Economos, C. D. (2010). Parent behavior and child weight status among a diverse group of underserved rural families. Appetite, 54(2), 369-377.

Hollis, S., \& Campbell, F. (1999). What is meant by intention to treat analysis? Survey of published randomised controlled trials. British Medical Journal, 319(7211), 670-674.

Hughes, S. O., Patrick, H., Power, T. G., Fisher, J. O., Anderson, C. B., \& Nicklas, T. A. (2007). The impact of child care providers' feeding on children's food consumption. Journal of Developmental and Behavioral Pediatrics, 28(2), 100-107.

Hughes, S. O., Power, T. G., Orlet Fisher, J., Mueller, S., \& Nicklas, T. A. (2005). Revisiting a neglected construct: parenting styles in a child-feeding context. Appetite, 44(1), 83-92.

Hughes, S. O., Shewchuk, R. M., Baskin, M. L., Nicklas, T. A., \& Qu, H. (2008). Indulgent feeding style and children's weight status in preschool. Journal of Developmental and Behavioral Pediatrics, 29(5), 403410.

Humenikova, L., \& Gates, G. E. (2008). Social and physical environmental factors and child overweight in a sample of American and Czech school-aged children: a pilot study. Journal of Nutrition Education and Behavior, 40(4), 251-257.

Hunt, C. (2003). The parenting puzzle: how to get the best out of family life. Oxford, UK: Family Links.

Israel, A. C., Guile, C. A., Baker, J. E., \& Silverman, W. K. (1994). An evaluation of enhanced self-regulation training in the treatment of childhood obesity. Journal of Pediatric Psychology, 19(6), 737-749.

Israel, A. C., Stolmaker, L., \& Andrian, C. A. (1985). The effects of training parents in general child management skills on a behavioral weight loss program for children. Behavior Therapy, 16(2), 169180.

Jackson, C., Bee -Gates, D. J., \& Henriksen, L. (1994). Authoritative parenting, child competencies, and initiation of cigarette smoking. Health Education Quarterly, 21(1), 103-116.

Jackson, D., Wilkes, L., \& McDonald, G. (2007). 'If I was in my daughter's body I'd be feeling devastated': women's experiences of mothering an overweight or obese child. Journal of Child Health Care, 11(1), 29-39. 


\section{0 | REFERENCES}

Jago, R., Steeds, J. K., Bentley, G. F., Sebire, S. J., Lucas, P. J., Fox, K. R., Stewart-Brown, S., \& Turner, K. M. (2012). Designing a physical activity parenting course: parental views on recruitment, content and delivery. BMC Public Health, 12, 356.

Jain, A., Sherman, S. N., Chamberlin, L. A., Carter, Y., Powers, S. W., \& Whitaker, R. C. (2001). Why don't lowincome mothers worry about their preschoolers being overweight? Pediatrics, 107(5), 1138-1146.

Janicke, D. M., Sallinen, B. J., Perri, M. G., Lutes, L. D., Silverstein, J. H., Huerta, M. G., \& Guion, L. A. (2008). Sensible treatment of obesity in rural youth (STORY): design and methods. Contemporary Clinical Trials, 29(2), 270-280.

Jansen, M. W., De Vries, N. K., Kok, G., \& Van Oers, H. A. (2008). Collaboration between practice, policy and research in local public health in the Netherlands. Health Policy, 86(2-3), 295-307.

Janssen, I., Katzmarzyk, P. T., Boyce, W. F., Vereecken, C., Mulvihill, C., Roberts, C., Currie, C., \& Pickett, W. (2005). Comparison of overweight and obesity prevalence in school-aged youth from 34 countries and their relationships with physical activity and dietary patterns. Obesity Reviews, 6(2), 123-132.

Jebb, S. A. (2005). Dietary strategies for the prevention of obesity. Proceedings of the Nutrition Society, 64(2), 217-227.

Jelalian, E., Boergers, J., Alday, C. S., \& Frank, R. (2003). Survey of physician attitudes and practices related to pediatric obesity. Clinical Pediatrics, 42(3), 235-245.

Johnston, C., \& Mash, E. J. (1989). A measure of parenting satisfaction and efficacy. Journal of Clinical Child Psychology, 18(2), 167-175.

Jones, T. L., \& Prinz, R. J. (2005). Potential roles of parental self-efficacy in parent and child adjustment: a review. Clinical Psychology Review, 25(3), 341-363.

Joyce, J. L., \& Zimmer-Gembeck, M. J. (2009). Parent feeding restriction and child weight. The mediating role of child disinhibited eating and the moderating role of the parenting context. Appetite, 52(3), 726-734.

Kalarchian, M. A., Levine, M. D., Arslanian, S. A., Ewing, L. J., Houck, P. R., Cheng, Y., Ringham, R. M., Sheets, C. A., \& Marcus, M. D. (2009). Family-based treatment of severe pediatric obesity: randomized, controlled trial. Pediatrics, 124(4), 1060-1068.

Kelder, S. H., Perry, C. L., Klepp, K. I., \& Lytle, L. L. (1994). Longitudinal tracking of adolescent smoking, physical activity, and food choice behaviors. American Journal of Public Health, 84(7), 1121-1126.

Kim, M. J. (2006). Parenting style and older children's and young adolescents dietary intake and nutritional status. Texas A\&M University.

Kim, M. J., McIntosh, W. A., Anding, J., Kubena, K. S., Reed, D. B., \& Moon, G. S. (2008). Perceived parenting behaviours predict young adolescents' nutritional intake and body fatness. Maternal \& Child Nutrition, 4(4), 287-303.

Kitzmann, K. M., \& Beech, B. M. (2006). Family-based interventions for pediatric obesity: methodological and conceptual challenges from family psychology. Journal of Family Psychology, 20(2), 175-189.

Kitzmann, K. M., Dalton, W. T., \& Buscemi, J. (2008). Beyond parenting practices: family context and the treatment of pediatric obesity. Family Relations, 57(1), 13-23.

Kitzmann, K. M., Stanley, C. M., Dalton, W. T., Beech, B. M., Reeves, T. P., Buscemi, J., Egli, C. J., Gamble, H. L., \& Midgett, E. L. (2010). Lifestyle interventions for youth who are overweight: A meta-analytic review. Health Psychology, 29(1), 91-101.

Knowlden, A. P., \& Sharma, M. (2012). Systematic review of family and home-based interventions targeting paediatric overweight and obesity. Obesity Reviews, 13(6), 499-508.

Koepsell, T. D., Zatzick, D. F., \& Rivara, F. P. (2011). Estimating the population impact of preventive interventions from randomized trials. American Journal of Preventive Medicine, 40(2), 191-198.

Kremers, S., Martens, M., Reubsaet, A., De Weerdt, I., De Vries, N., \& Jonkers, R. (2008). Programmeringstudie overgewicht. In ZonMW (Ed.): Rescon, Universiteit Maastricht.

Kremers, S. P., Brug, J., De Vries, H., \& Engels, R. C. (2003). Parenting style and adolescent fruit consumption. Appetite, 41(1), 43-50. 
Kremers, S. P., De Bruijn, G. J., Visscher, T. L., Van Mechelen, W., De Vries, N. K., \& Brug, J. (2006). Environmental influences on energy balance-related behaviors: a dual-process view. International Journal of Behavioural Nutrition and Physical Activity, 3, 9.

Kremers, S. P., Visscher, T. L., Seidell, J. C., Van Mechelen, W., \& Brug, J. (2005). Cognitive determinants of energy balance-related behaviours: measurement issues. Sports Medicine, 35(11), 923-933.

Kroke, A., Strathmann, S., \& Gunther, A. L. (2006). Maternal perceptions of her child's body weight in infancy and early childhood and their relation to body weight status at age 7. European Journal of Pediatrics, 165(12), 875-883.

Kubik, M. Y., Story, M., \& Davey, C. (2007). Obesity prevention in schools: current role and future practice of school nurses. Preventive Medicine, 44(6), 504-507.

Kummeling, I., Thijs, C., Penders, J., Snijders, B. E., Stelma, F., Reimerink, J., Koopmans, M., Dagnelie, P. C., Huber, M., Jansen, M. C., De Bie, R., \& Van den Brandt, P. A. (2005). Etiology of atopy in infancy: the KOALA Birth Cohort Study. Pediatric Allergy and Immunology, 16(8), 679-684.

Lamberts, P. H. G., Steenbakkers, M., Thijssen, N. H. B., Backus-Mujakovic, S., De Vreede, J. J. M., Hajema, K. J., \& Poos, M. J. J. C. (2010). Een gezonde kijk op Zuid-Limburg. Regionale Volksgezondheid Toekomst Verkenning. 2010. Geleen: GGD Zuid Limburg.

Lamborn, S. D., Mounts, N. S., Steinberg, L., \& Dornbusch, S. M. (1991). Patterns of competence and adjustment among adolescents from authoritative, authoritarian, indulgent, and neglectful families. Child Development, 62(5), 1049-1065.

Lee, R. E., McGinnis, K. A., Sallis, J. F., Castro, C. M., Chen, A. H., \& Hickmann, S. A. (1997). Active vs. passive methods of recruiting ethnic minority women to a health promotion program. Annals of Behavioral Medicine, 19(4), 378-384.

Leufkens, K., \& Souren, M. (2011). Bevolkingstrends, 1e kwartaal 2011: CBS.

Lipsey, M. W. (1990). Design sensitivity: statistical power for experimental research. Newbury Park, California: Sage Publications.

Lissau, I., \& Sorensen, T. I. (1994). Parental neglect during childhood and increased risk of obesity in young adulthood. Lancet, 343(8893), 324-327.

Lohaus, A., Vierhaus, M., \& Ball, J. (2009). Parenting styles and health-related behavior in childhood and early adolescence. The Journal of Early Adolescence, 29(4), 449-475.

Lozano, P., McPhillips, H. A., Hartzler, B., Robertson, A. S., Runkle, C., Scholz, K. A., Stout, J. W., \& Kieckhefer, G. M. (2010). Randomized trial of teaching brief motivational interviewing to pediatric trainees to promote healthy behaviors in families. Archives of Pediatric and Adolescent Medicine, 164(6), 561-566.

Ludrosky, J. M. (2005). Childhood obesity: multifactoral determinants of child weight and eating behaviors: Miami University.

Lytle, L. A., Varnell, S., Murray, D. M., Story, M., Perry, C., Birnbaum, A. S., \& Kubik, M. Y. (2003). Predicting adolescents' intake of fruits and vegetables. Journal of Nutrition Education and Behavior, 35(4), 170175.

Maccoby, E. E., \& Martin, J. A. (1983). Socialization in the context of the family: parent-child interaction. Handbook of child psychology, 4, 1-101.

Magarey, A. M., Daniels, L. A., Boulton, T. J., \& Cockington, R. A. (2003). Predicting obesity in early adulthood from childhood and parental obesity. International Journal of Obesity, 27(4), 505-513.

Magarey, A. M., Perry, R. A., Baur, L. A., Steinbeck, K. S., Sawyer, M., Hills, A. P., Wilson, G., Lee, A., \& Daniels, L. A. (2011). A parent-led family-focused treatment program for overweight children aged 5 to 9 years: the PEACH RCT. Pediatrics, 127(2), 214-222.

Malina, R. M., \& Little, B. B. (2008). Physical activity: the present in the context of the past. American Journal of Human Biology, 20(4), 373-391.

Mamun, A. A., McDermott, B. M., O'Callaghan, M. J., Najman, J. M., \& Williams, G. M. (2008). Predictors of maternal misclassifications of their offspring's weight status: a longitudinal study. International Journal of Obesity, 32(1), 48-54. 


\section{REFERENCES}

Manios, Y., Kondaki, K., Kourlaba, G., Vasilopoulou, E., \& Grammatikaki, E. (2009). Maternal perceptions of their child's weight status: the GENESIS study. Public Health Nutrition, 12(8), 1099-1105.

Matheson, D. M., Robinson, T. N., Varady, A., \& Killen, J. D. (2006). Do Mexican-American mothers' foodrelated parenting practices influence their children's weight and dietary intake? Journal of the American Dietetic Association, 106(11), 1861-1865.

Maynard, L. M., Galuska, D. A., Blanck, H. M., \& Serdula, M. K. (2003). Maternal perceptions of weight status of children. Pediatrics, 111(5 Part 2), 1226-1231.

Mendelson, B. K., White, D. R., \& Schliecker, E. (1995). Adolescents' weight, sex, and family functioning. International Journal of Eating Disorders, 17(1), 73-79.

Mihalopoulos, C., Sanders, M. R., Turner, K. M., Murphy-Brennan, M., \& Carter, R. (2007). Does the Triple PPositive Parenting Program provide value for money? Australasian Psychiatry, 41(3), 239-246.

Mihalopoulos, C., Vos, T., Pirkis, J., \& Carter, R. (2011). The economic analysis of prevention in mental health programs. Annual Review of Clinical Psychology, 7, 169-201.

Miller Perrin, E., Flower, K. B., Garrett, J., \& Ammerman, A. S. (2005). Preventing and treating obesity: pediatricians' self-efficacy, barriers, resources, and advocacy. Ambulatory Pediatrics, 5(3), 150-156.

Miller, W. R., \& Rollnick, S. (2002). Motivational interviewing: Preparing people for change (Vol. Second edition). New York: The Guilford Press.

Mitchell, S., Brennan, L., Hayes, L., \& Miles, C. L. (2009). Maternal psychosocial predictors of controlling parental feeding styles and practices. Appetite, 53(3), 384-389.

Moens, E., \& Braet, C. (2012). Training parents of overweight children in parenting skills: a 12-month evaluation. Behavioural and Cognitive Psychotherapy, 40(1), 1-18.

Moens, E., Braet, C., \& Soetens, B. (2007). Observation of family functioning at mealtime: a comparison between families of children with and without overweight. Journal of Pediatric Psychology, 32(1), 5263.

Montgomery, C., Jackson, D. M., Kelly, L. A., \& Reilly, J. J. (2006). Parental feeding style, energy intake and weight status in young Scottish children. British Journal of Nutrition, 96(06), 1149-1153.

Moyers, P., Bugle, L., \& Jackson, E. (2005). Perceptions of school nurses regarding obesity in school-age children. Journal of School Nursing, 21(2), 86-93.

Mullis, F. (1999). Active parenting: an evaluation of two Adlerian parent education programs. Journal of Individual Psychology, 55(2), 225-232.

Musher-Eizenman, D., \& Holub, S. (2006). Children's eating in the absence of hunger: the role of restrictive feeding practices. Childhood Obesity and Health Research, 135-156.

Musher-Eizenman, D. R., De Lauzon-Guillain, B., Holub, S. C., Leporc, E., \& Charles, M. A. (2009). Child and parent characteristics related to parental feeding practices. A cross-cultural examination in the US and France. Appetite, 52(1), 89-95.

Must, A., \& Strauss, R. S. (1999). Risks and consequences of childhood and adolescent obesity. International Journal of Obesity, 23 Suppl 2, S2-11.

Mustillo, S., Worthman, C., Erkanli, A., Keeler, G., Angold, A., \& Costello, E. J. (2003). Obesity and psychiatric disorder: developmental trajectories. Pediatrics, 111(4 Pt 1), 851-859.

Nelson, M., \& Davis, M. C. (2013). Development and preliminary validation of the Parent Efficacy for Child Healthy Weight Behaviour Scale. Journal of Health Psychology, 18(2), 282-291.

Newman, K., Harrison, L., Dashiff, C., \& Davies, S. (2008). Relationships between parenting styles and risk behaviors in adolescent health: an integrative literature review. Revista Latino-Americana de Enfermagem, 16(1), 142-150.

Niemeier, B. S., Hektner, J. M., \& Enger, K. B. (2012). Parent participation in weight-related health interventions for children and adolescents: a systematic review and meta-analysis. Preventive Medicine, 55(1), 3-13. 
Nowak, C., \& Heinrichs, N. (2008). A comprehensive meta-analysis of Triple P-Positive Parenting Program using hierarchical linear modeling: effectiveness and moderating variables. Clinical Child and Family Psychology Review, 11(3), 114-144.

Nowicka, P., \& Flodmark, C. E. (2008). Family in pediatric obesity management: a literature review. International Journal of Pediatric Obesity, 3 Suppl 1, 44-50.

Nowicka, P., Pietrobelli, A., \& Flodmark, C. E. (2007). Low-intensity family therapy intervention is useful in a clinical setting to treat obese and extremely obese children. International Journal of Pediatric Obesity, 2(4), 211-217.

Olvera, N., \& Power, T. G. (2010). Brief report: parenting styles and obesity in Mexican American children: a longitudinal study. Journal of Pediatric Psychology, 35(3), 243-249.

Ostbye, T., Krause, K. M., Stroo, M., Lovelady, C. A., Evenson, K. R., Peterson, B. L., Bastian, L. A., Swamy, G. K., West, D. G., Brouwer, R. J., \& Zucker, N. L. (2012). Parent-focused change to prevent obesity in preschoolers: results from the KAN-DO study. Preventive Medicine, 55(3), 188-195.

Oude Luttikhuis, H., Baur, L., Jansen, H., Shrewsbury, V. A., O'Malley, C., Stolk, R. P., \& Summerbell, C. D. (2009). Interventions for treating obesity in children. Cochrane Database Systematic Reviews(1), CD001872.

Oude Luttikhuis, H. G., Stolk, R. P., \& Sauer, P. J. (2010). How do parents of 4- to 5-year-old children perceive the weight of their children? Acta Paediatrica, 99(2), 263-267.

Owen, N., Healy, G. N., Matthews, C. E., \& Dunstan, D. W. (2010). Too much sitting: the population health science of sedentary behavior. Exercise and Sport Sciences Reviews, 38(3), 105-113.

Pagnini, D. L., Wilkenfeld, R. L., King, L. A., Booth, M. L., \& Booth, S. L. (2007). Mothers of pre-school children talk about childhood overweight and obesity: The Weight Of Opinion Study. Journal of Paediatrics and Child Health, 43(12), 806-810.

Parker, G., Tupling, H., \& Brown, L. (1979). A parental bonding instrument. British Journal of Medical Psychology, 52(1), 1-10.

Parry, L. L., Netuveli, G., Parry, J., \& Saxena, S. (2008). A systematic review of parental perception of overweight status in children. The Journal of Ambulatory Care Management, 31(3), 253-268.

Patrick, H., Nicklas, T. A., Hughes, S. O., \& Morales, M. (2005). The benefits of authoritative feeding style: caregiver feeding styles and children's food consumption patterns. Appetite, 44(2), 243-249.

Patterson, G. R. (1976). Living with children: new methods for parents and teachers (Revised). Champaign, Illinois: Research Press.

Patterson, G. R. (1982). Coercive family process. Eugene, OR: Castalia Press.

Patterson, G. R., \& Gullion, M. E. (1968). Living with children: new methods for parents and teachers. Champaign, Illinois: Research Press.

Pearson, N., Atkin, A. J., Biddle, S. J., Gorely, T., \& Edwardson, C. (2010). Parenting styles, family structure and adolescent dietary behaviour. Public Health Nutrition, 13(8), 1245-1253.

Perez-Escamilla, R., Obbagy, J. E., Altman, J. M., Essery, E. V., McGrane, M. M., Wong, Y. P., Spahn, J. M., \& Williams, C. L. (2012). Dietary energy density and body weight in adults and children: a systematic review. Journal of the Academy of Nutrition and Dietetics, 112(5), 671-684.

Polit, D. F., \& Beck, C. T. (2008). Nursing research: Generating and assessing evidence for nursing practice: Lippincott Williams \& Wilkins.

Power, T. G. (1993). A research manual. . University of Houston. Houson, TX.

Prinz, R. J., \& Sanders, M. R. (2007). Adopting a population-level approach to parenting and family support interventions. Clinical Psychology Review, 27(6), 739-749.

Pulkkinen, L. (1982). Self-control and continuity from childhood to late adolescence. Life-span development and behavior, 4, 63-105.

Raynor, H. A., Osterholt, K. M., Hart, C. N., Jelalian, E., Vivier, P., \& Wing, R. R. (2009). Evaluation of active and passive recruitment methods used in randomized controlled trials targeting pediatric obesity. International Journal of Pediatric Obesity, 4(4), 224-232. 


\section{REFERENCES}

Redsell, S. A., Atkinson, P. J., Nathan, D., Siriwardena, A. N., Swift, J. A., \& Glazebrook, C. (2011). Preventing childhood obesity during infancy in UK primary care: a mixed-methods study of HCPs' knowledge, beliefs and practice. BMC Family Practice, 12, 54.

Reineke, P. R. (2008). The relationship between parenting style and child weight. New York University.

Reitman, D., Rhode, P. C., Hupp, S. D. A., \& Altobello, C. (2002). Development and validation of the parental authority Questionnaireâ€"Revised. Journal of Psychopathology and Behavioral Assessment, 24(2), 119-127.

Rennie, K. L., Johnson, L., \& Jebb, S. A. (2005). Behavioural determinants of obesity. Best Practice and Research: Clinical Endocrinology \& Metabolism, 19(3), 343-358.

Rhee, K. (2008). Childhood overweight and the relationship between parent behaviors, parenting style, and family functioning. The ANNALS of the American Academy of Political and Social Science, 615(1), 11.

Rhee, K. E., Lumeng, J. C., Appugliese, D. P., Kaciroti, N., \& Bradley, R. H. (2006). Parenting styles and overweight status in first grade. Pediatrics, 117(6), 2047-2054.

Rice, J., Thombs, D., Leach, R., \& Rehm, R. (2008). Successes and barriers for a youth weight-management program. Clinical Pediatrics, 47(2), 143-147.

Robertson, W., Friede, T., Blissett, J., Rudolf, M. C. J., Wallis, M., \& Stewart-Brown, S. (2008). Pilot of "Families for Health": community-based family intervention for obesity. Archives of Disease in Childhood, 93(11), 921-928.

Robertson, W., Stewart-Brown, S., Wilcock, E., Oldfield, M., \& Thorogood, M. (2011). Utility of accelerometers to measure physical activity in children attending an obesity treatment intervention. Journal of Obesity, 2011.

Robinson, T. N. (1999). Reducing children's television viewing to prevent obesity. A randomized controlled trial. Journal of the American Medical Association, 282(16), 1561-1567.

Robinson, C., Mandleco, B., Olsen, S. F., \& Hart, C. (2001). The parenting styles and dimensions questionnaire (PSDQ) Handbook of family measurement techniques (Vol. 3, pp. 319-321). Thousands Oaks, CA.

Robinson, C. C., Mandleco, B., Olsen, S. F., \& Hart, C. (1995). Authoritative, authoritarian, and permissive parenting practices: Development of a new measure. Psychological Reports, 77(3), 819-830.

Rodenburg, G., Kremers, S. P., Oenema, A., \& Van de Mheen, D. (2011). Psychological control by parents is associated with a higher child weight. International Journal of Pediatric Obesity, 6(5-6), 442-449.

Rodenburg, G., Kremers, S. P., Oenema, A., \& Van de Mheen, D. (2012). Associations of children's appetitive traits with weight and dietary behaviours in the context of general parenting. PLoS One, 7(12), e50642.

Rogers, E. M. (2003). Diffusion of innovations (Vol. Fifith edition). New York: Free Press.

Rohner, R. P. (1986). The warmth dimension: Foundations of parental acceptance-rejection theory. Newbury Park CA: Sage Publications.

Rothbart, M. K., Ahadi, S. A., Hershey, K. L., \& Fisher, P. (2001). Investigations of temperament at three to seven years: the Children's Behavior Questionnaire. Child Development, 72(5), 1394-1408.

Rutter, M. (1985). Family and school influences on behavioural development. Journal of Child Psychology and Psychiatry, 26(3), 349-368.

Sanders, M., Prior, J., \& Ralph, A. (2009). An evaluation of a brief universal seminar series on positive parenting: a feasibility study. Journal of Children's Services, 4(1), 4-20.

Sanders, M. R. (1992). Enhancing the impact of behavioural family intervention with children: Emerging perspectives. Behaviour Change.

Sanders, M. R. (1996). New directions in behavioral family intervention with children. Advances in clinical child psychology (pp. 283-330): Springer.

Sanders, M. R. (2012). Development, evaluation, and multinational dissemination of the Triple P-Positive Parenting Program. Annual Review of Clinical Psychology, 8, 345-379.

Sanders, M. R., Markie-Dadds, C., \& Turner, K. M. T. (2003). Theoretical, scientific and clinical foundations of the Triple P-Positive Parenting Program: a population approach to the promotion of parenting competence. Parenting Research and Practice Monograph, 1, 1-24. 
Sanders, M. R., \& Mazzucchelli, T. G. (2013). The promotion of self-regulation through parenting interventions. Clinical Child and Family Psychology Review, 1-17.

Sandler, I. N., Schoenfelder, E. N., Wolchik, S. A., \& Mackinnon, D. P. (2011). Long-term impact of prevention programs to promote effective parenting: lasting effects but uncertain processes. Annual Review of Psychology, 62, 299-329.

Saunders, K. L. (2007). Preventing obesity in pre-school children: a literature review. Journal of Public Health, 29(4), 368-375.

Schaefer, E. S. (1959). A circumplex model for maternal behavior. Journal of Abnormal and Social Psychology, 59, 226-235.

Schmitz, K. H., Lytle, L. A., Phillips, G. A., Murray, D. M., Birnbaum, A. S., \& Kubik, M. Y. (2002). Psychosocial correlates of physical activity and sedentary leisure habits in young adolescents: the Teens Eating for Energy and Nutrition at School study. Preventive Medicine, 34(2), 266-278.

Schokker, D. F., Hekkert, K. D., Kocken, P. L., Van de Brink, C. L., \& De Vries, S. I. (2012). Meten van lichamelijke activiteit van kinderen: vragenlijsten vergeleken met versnellingsmeter. Tijdschrift voor gezondheidswetenschappen, 90(7), 434-441.

Scholtens, S., Brunekreef, B., Visscher, T. L., Smit, H. A., Kerkhof, M., De Jongste, J. C., Gerritsen, J., \& Wijga, A. H. (2007). Reported versus measured body weight and height of 4-year-old children and the prevalence of overweight. European Journal of Public Health, 17(4), 369-374.

Schonbeck, Y., Talma, H., Van Dommelen, P., Bakker, B., Buitendijk, S. E., Hirasing, R. A., \& Van Buuren, S. (2011). Increase in prevalence of overweight in Dutch children and adolescents: a comparison of nationwide growth studies in 1980, 1997 and 2009. PLoS One, 6(11), e27608.

Schulz, D. N., Candel, M. J., Kremers, S. P., Reinwand, D. A., Jander, A., \& De Vries, H. (2013). Effects of a web-based tailored intervention to reduce alcohol consumption in adults: randomized controlled trial. J Med Internet Res, 15(9), e206.

Schwartz, R. P., Hamre, R., Dietz, W. H., Wasserman, R. C., Slora, E. J., Myers, E. F., Sullivan, S., Rockett, H., Thoma, K. A., Dumitru, G., \& Resnicow, K. A. (2007). Office-based motivational interviewing to prevent childhood obesity: a feasibility study. Archives of Pediatric and Adolescent Medicine, 161(5), 495-501.

Seidell, J., De Beer, J., \& Kuijpers, T. (2008). Richtlijn 'Diagnostiek en behandeling van obesitas bij volwassenen en kinderen'. Nederlands tijdschrift voor geneeskunde, 152(38), 2071-2076.

Senden, P. P. F. M., \& Krumeich, A. J. S. M. (2011). Follow the folder! Werving en promotie van de RealFitcursus voor jongeren/adolescenten met (beginnend) overgewicht. Tijdschrift voor gezondheidswetenschappen, 4, 201-205.

Shelton, D., Le Gros, K., Norton, L., Stanton Cook, S., Morgan, J., \& Masterman, P. (2007). Randomised controlled trial: A parent-based group education programme for overweight children. Journal of Paediatric Child Health, 43(12), 799-805.

Singh, A. S., Mulder, C., Twisk, J. W. R., Van Mechelen, W., \& Chinapaw, M. J. M. (2008). Tracking of childhood overweight into adulthood: a systematic review of the literature. Obesity Reviews, 9(5), 474-488.

Skelton, J. A., \& Beech, B. M. (2011). Attrition in paediatric weight management: a review of the literature and new directions. Obesity Reviews, 12(5), e273-281.

Skinner, B. F. (1953). Science and human behavior. New York: Macmillan Inc.

Skinner, E., Johnson, S., \& Snyder, T. (2005). Six dimensions of parenting: A motivational model. Parenting: Science and Practice, 5(2), 175-235.

Skouteris, H., McCabe, M., Swinburn, B., Newgreen, V., Sacher, P., \& Chadwick, P. (2011). Parental influence and obesity prevention in pre-schoolers: a systematic review of interventions. Obesity Reviews, 12(5), 315-328.

Sleddens, E. F., Gerards, S. M., Thijs, C., De Vries, N. K., \& Kremers, S. P. (2011). General parenting, childhood overweight and obesity-inducing behaviors: a review. International Journal of Pediatric Obesity, 6(2-2), e12-27. 


\section{6 | REFERENCES}

Sleddens, E. F., Kremers, S. P., De Vries, N. K., \& Thijs, C. (2010). Relationship between parental feeding styles and eating behaviours of Dutch children aged 6-7. Appetite, 54(1), 30-36.

Sleddens, E. F. C., Kremers, S. P. J., Stafleu, A., Dagnelie, P. C., De Vries, N. K., \& Thijs, C. (under review). Food parenting practices and child dietary behavior: Prospective relations and the moderating role of general parenting.

Smith, B. J., Grunseit, A., Hardy, L. L., King, L., Wolfenden, L., \& Milat, A. (2010). Parental influences on child physical activity and screen viewing time: a population based study. BMC Public Health, 10, 593.

Spera, C. (2005). A review of the relationship among parenting practices, parenting styles, and adolescent school achievement. Educational Psychology Review, 17(2), 125-146.

Spivack, J. G., Swietlik, M., Alessandrini, E., \& Faith, M. S. (2010). Primary care providers' knowledge, practices, and perceived barriers to the treatment and prevention of childhood obesity. Obesity, 18(7), 1341-1347.

Spoth, R., Redmond, C., \& Shin, C. (1998). Direct and indirect latent-variable parenting outcomes of two universal family-focused preventive interventions: extending a public health-oriented research base. Journal of Consulting and Clinical Psychology, 66(2), 385-399.

Stark, L. J., Spear, S., Boles, R., Kuhl, E., Ratcliff, M., Scharf, C., Bolling, C., \& Rausch, J. (2011). A pilot randomized controlled trial of a clinic and home-based behavioral intervention to decrease obesity in preschoolers. Obesity, 19(1), 134-141.

Steele, R. G., Wu, Y. P., Jensen, C. D., Pankey, S., Davis, A. M., \& Aylward, B. S. (2011). School nurses' perceived barriers to discussing weight with children and their families: a qualitative approach. Journal of School Health, 81(3), 128-137.

Stein, R. I., Epstein, L. H., Raynor, H. A., Kilanowski, C. K., \& Paluch, R. A. (2005). The influence of parenting change on pediatric weight control. Obesity Research, 13(10), 1749-1755.

Steinberg, L., Elmen, J. D., \& Mounts, N. S. (1989). Authoritative parenting, psychosocial maturity, and academic success among adolescents. Child Development, 60(6), 1424-1436.

Stenhammar, C., Olsson, G., Bahmanyar, S., Hulting, A. L., Wettergren, B., Edlund, B., \& Montgomery, S. (2010). Family stress and BMI in young children. Acta Paediatrica, 99(8), 1205-1212.

Stice, E., Shaw, H., \& Marti, C. N. (2006). A meta-analytic review of obesity prevention programs for children and adolescents: the skinny on interventions that work. Psychological Bulletin, 132(5), 667-691.

Story, M. T., Neumark-Stzainer, D. R., Sherwood, N. E., Holt, K., Sofka, D., Trowbridge, F. L., \& Barlow, S. E. (2002). Management of child and adolescent obesity: attitudes, barriers, skills, and training needs among health care professionals. Pediatrics, 110(1), 210-214.

Strauss, R. S. (2000). Childhood obesity and self-esteem. Pediatrics, 105(1), e15.

Strauss, R. S., \& Knight, J. (1999). Influence of the home environment on the development of obesity in children. Pediatrics, 103(6), e85.

Suarez, M., \& Mullins, S. (2008). Motivational interviewing and pediatric health behavior interventions. Journal of Developmental and Behavioral Pediatrics, 29(5), 417-428.

Summerbell, C., Waters, E., Edmunds, L., Kelly, S., Brown, T., \& Campbell, K. (2005). Interventions for preventing obesity in children. Cochrane Database of Systematic Reviews, 3(3).

Summerbell, C. D., Moore, H. J., Vogele, C., Kreichauf, S., Wildgruber, A., Manios, Y., Douthwaite, W., Nixon, C. A., \& Gibson, E. L. (2012). Evidence-based recommendations for the development of obesity prevention programs targeted at preschool children. Obesity Reviews, 13 Suppl 1, 129-132.

Sung-Chan, P., Sung, Y. W., Zhao, X., \& Brownson, R. C. (2013). Family-based models for childhood-obesity intervention: a systematic review of randomized controlled trials. Obesity Reviews, 14, 265-278.

Swinburn, B., Egger, G., \& Raza, F. (1999). Dissecting obesogenic environments: the development and application of a framework for identifying and prioritizing environmental interventions for obesity. Preventive Medicine, 29(6 Pt 1), 563-570. 
Taveras, E. M., Gortmaker, S. L., Hohman, K. H., Horan, C. M., Kleinman, K. P., Mitchell, K., Price, S., Prosser, L. A., Rifas Shiman, S. L., \& Gillman, M. W. (2011). Randomized Controlled Trial to improve primary care to prevent and manage childhood obesity: The high five for kids study. Archives of Pediatric and Adolescent Medicine.

Te Velde, S. J., Van Nassau, F., Uijtdewilligen, L., Van Stralen, M. M., Cardon, G., De Craemer, M., Manios, Y., Brug, J., \& Chinapaw, M. J. (2012). Energy balance-related behaviours associated with overweight and obesity in preschool children: a systematic review of prospective studies. Obesity Reviews, 13 Suppl 1, 56-74.

Tellegen, C. L., \& Sanders, M. R. (2013). Stepping Stones Triple P-Positive Parenting Program for children with disability: A systematic review and meta-analysis. Research in Developmental Disabilities, 34(5), 15561571.

Thomas, R., \& Zimmer-Gembeck, M. J. (2007). Behavioral outcomes of parent-child interaction therapy and Triple P-Positive Parenting Program: A review and meta-analysis. Journal of Abnormal Child Psychology, 35(3), 475-495.

Timmermans, S. H., Mommers, M., Gubbels, J. S., Kremers, S. P., Stafleu, A., Stehouwer, C. D., Prins, M. H., Penders, J., \& Thijs, C. (2013). Maternal smoking during pregnancy and childhood overweight and fat distribution: the KOALA Birth Cohort Study. Pediatric Obesity.

Topham, G. L., Page, M. C., Hubbs Tait, L., Rutledge, J. M., Kennedy, T. S., Shriver, L., \& Harrist, A. W. (2010). Maternal depression and socio-economic status moderate the parenting style/child obesity association. Public Health Nutrition, 13(8), 1237-1244.

Towns, N., \& D'Auria, J. (2009). Parental perceptions of their child's overweight: an integrative review of the literature. Journal of Pediatric Nursing, 24(2), 115-130.

Trombini, E., Baldaro, B., Bertaccini, R., Mattei, C., Montebarocci, O., \& Rossi, N. (2003). Maternal attitudes and attachment styles in mothers of obese children. Perceptual \& Motor Skills, 97(2), 613-620.

Trost, S. G. (2007). State of the art reviews: measurement of physical activity in children and adolescents. American Journal of Lifestyle Medicine, 1(4), 299-314.

Turner, H. M., Rose, K. S., \& Cooper, M. J. (2005). Schema and parental bonding in overweight and nonoverweight female adolescents. International Journal of Obesity, 29(4), 381-387.

Turner, K. M., Shield, J. P., \& Salisbury, C. (2009). Practitioners' views on managing childhood obesity in primary care: a qualitative study. British Journal of General Practice, 59(568), 856-862.

Van Buuren, S. (2012). Flexible imputation of missing data: CRC press.

Van den Hurk, K., Van Dommelen, P., Van Buuren, S., Verkerk, P. H., \& Hirasing, R. A. (2007). Prevalence of overweight and obesity in the Netherlands in 2003 compared to 1980 and 1997. Archives of Disease in Childhood, 92, 992-995.

Van der Horst, K., Kremers, S., Ferreira, I., Singh, A., Oenema, A., \& Brug, J. (2007). Perceived parenting style and practices and the consumption of sugar-sweetened beverages by adolescents. Health Education Research, 22(2), 295-304.

Van Grieken, A., Veldhuis, L., Renders, C. M., Borsboom, G. J., Van der Wouden, J. C., Hirasing, R. A., \& Raat, H. (2013). Population-based childhood overweight prevention: outcomes of the 'be active, eat right' study. PLoS One, 8(5), e65376.

Van Rossum, C. T. M., Fransen, H. P., Verkaik-Kloosterman, J., Buurma-Rethans, E. J. M., \& Ocké, M. C. (2011). Dutch National Food Consumption Survey 2007-2010. Bilthoven: National Institute for Public Health and the Environment.

Van Wieringen, J. C. M., \& Van Wieren, S. (2008). Wat is het bereik van de jeugdgezondheidszorg en wat zijn de effecten? Volksgezondheid Toekomst Verkenning, Nationaal Kompas Volksgezondheid. Retrieved 23 september, 2008, from http://www.nationaalkompas.nl/zorg/publieke-gezondheidszorg/jeugdgezondheidszorg/bereik-effecten/ 


\section{REFERENCES}

Veldhuis, L., Struijk, M. K., Kroeze, W., Heijnen, J. W. M., Oenema, A., Renders, C. M., HiraSing, R. A., \& Raat, H. (2009a). Feasibility of an overweight prevention protocol in Youth Health Care. Paper presented at the ECO, Amsterdam.

Veldhuis, L., Struijk, M. K., Kroeze, W., Oenema, A., Renders, C. M., Bulk-Bunschoten, A. M., Hirasing, R. A., \& Raat, H. (2009b). 'Be active, eat right', evaluation of an overweight prevention protocol among 5-yearold children: design of a cluster randomised controlled trial. BMC Public Health, 9, 177.

Ventura, A. K., \& Birch, L. L. (2008). Does parenting affect children's eating and weight status? International Journal of Behavioural Nutrition and Physical Activity, 5(1), 15.

Vereecken, C., Legiest, E., De Bourdeaudhuij, I., \& Maes, L. (2009). Associations between general parenting styles and specific food-related parenting practices and children's food consumption. American Journal of Health Promotion, 23(4), 233-240.

Verloove-Vanhorick, S. P., Borghuis, I. E., Juttmann, R. E., Lim-Feijen, J. F., Rieffe, J. A., Schulpen, T. W. J., Spierings, G. A. P., Wassenaar, J., De Winter, M., \& Zoomers, H. C. M. (2002). Basistakenpakket Jeugdgezondheidszorg 0-19 jaar: Ministerie van Volksgezondheid, Welzijn en Sport.

Visscher, T. L., \& Seidell, J. C. (2004). Time trends (1993-1997) and seasonal variation in body mass index and waist circumference in the Netherlands. International Journal of Obesity Related Metabolic Disorders, 28(10), 1309-1316.

Wake, M., Nicholson, J. M., Hardy, P., \& Smith, K. (2007). Preschooler obesity and parenting styles of mothers and fathers: Australian national population study. Pediatrics, 120(6), e1520-1527.

Walker, O., Strong, M., Atchinson, R., Saunders, J., \& Abbott, J. (2007). A qualitative study of primary care clinicians' views of treating childhood obesity. BMC Family Practice, 8, 50.

Wang, Y., \& Lobstein, T. (2006). Worldwide trends in childhood overweight and obesity. International Journal of Pediatric Obesity, 1(1), 11-25.

Wardle, J., \& Carnell, S. (2007). Parental feeding practices and children's weight. Acta Paediatrica, 96(454), 511.

Wardle, J., Guthrie, C. A., Sanderson, S., \& Rapoport, L. (2001). Development of the Children's Eating Behaviour Questionnaire. Journal of Child Psychology and Psychiatry, 42(7), 963-970.

Warren, J. M., Golley, R. K., Collins, C. E., Okely, A. D., Jones, R. A., Morgan, P. J., Perry, R. A., Baur, L. A., Steele, J. R., \& Magarey, A. M. (2007). Randomised controlled trials in overweight children: practicalities and realities. International Journal of Pediatric Obesity, 2(2), 73-85.

Warschburger, P., \& Kroller, K. (2009). Maternal perception of weight status and health risks associated with obesity in children. Pediatrics, 124(1), e60-68.

Warschburger, P., \& Kroller, K. (2012). Childhood overweight and obesity: maternal perceptions of the time for engaging in child weight management. BMC Public Health, 12(1), 295.

Washington, R. L. (2011). Childhood obesity: issues of weight bias. Preventing Chronic Disease, 8(5), A94.

Waters, E., De Silva-Sanigorski, A., Hall, B. J., Brown, T., Campbell, K. J., Gao, Y., Armstrong, R., Prosser, L., \& Summerbell, C. D. (2011). Interventions for preventing obesity in children. Cochrane Database of Systematic Reviews, 12.

Waylen, A., Stallard, N., \& Stewart-Brown, S. (2008). Parenting and health in mid-childhood: a longitudinal study. European Journal of Public Health, 18(3), 300-305.

West, F. (2007). The Lifestyle Triple P Project. Exploring the link between parenting and childhood obesity. (Doctor of Philosophy), University of Queensland.

West, F., Morawska, A., \& Joughin, K. (2010a). The Lifestyle Behaviour Checklist: evaluation of the factor structure. Child: Care, Health and Development, 36(4), 508-515.

West, F., \& Sanders, M. R. (2005). The Lifestyle Behaviour Checklist., Parenting \& Family Support Centre, University of Queensland, Brisbane, Australia.

West, F., \& Sanders, M. R. (2009). The Lifestyle Behaviour Checklist: a measure of weight-related problem behaviour in obese children. International Journal of Pediatric Obesity, 4(4), 266-273. 
West, F., \& Sanders, M. R. (Eds.). (2010). Facilitator's Manual for Group Lifestyle Triple P. Milton QLD: Triple P International.

West, F., Sanders, M. R., Cleghorn, G. J., \& Davies, P. S. (2010b). Randomised clinical trial of a family-based lifestyle intervention for childhood obesity involving parents as the exclusive agents of change. Behaviour Research and Therapy, 48(12), 1170-1179.

Wethington, H. R., Sherry, B., \& Polhamus, B. (2011). Physician practices related to use of BMI-for-age and counseling for childhood obesity prevention: a cross-sectional study. BMC Family Practice, 12, 80.

Whitaker, R. C., Wright, J. A., Pepe, M. S., Seidel, K. D., \& Dietz, W. H. (1997). Predicting obesity in young adulthood from childhood and parental obesity. The New England Journal of Medicine, 337(13), 869873.

White, I. R., Royston, P., \& Wood, A. M. (2011). Multiple imputation using chained equations: Issues and guidance for practice. Statistics in medicine, 30(4), 377-399.

World Health Organization. (2003). Joint WHO / FAO Expert Consultation: Diet, nutrition and the prevention of chronic diseases. Geneva: WHO.

World Health Organization. (2006). Obesity and Overweight. Geneva: WHO.

World Health Organization. (2011). Childhood overweight and obesity. Retrieved from the World Wide Web: http://www.who.int/dietphysicalactivity/childhood/en/

World Health Organization. (2013). Childhood overweight and obesity. Retrieved from the World Wide Web: http://www.who.int/dietphysicalactivity/childhood/en/

Willis, G. B. (1999). Cognitive Interviewing. A 'how to' guide: Research Triangle Institute.

Willis, T. A., George, J., Hunt, C., Roberts, K. P., Evans, C. E., Brown, R. E., \& Rudolf, M. C. (2013). Combating child obesity: impact of HENRY on parenting and family lifestyle. Pediatric Obesity.

Wilson, P., Rush, R., Hussey, S., Puckering, C., Sim, F., Allely, C. S., Doku, P., McConnachie, A., \& Gillberg, C. (2012). How evidence-based is an 'evidence-based parenting program'? A PRISMA systematic review and meta-analysis of Triple P. BMC Medicine, 10, 130.

Wolman, J., Skelly, E., Kolotourou, M., Lawson, M., \& Sacher, P. (2008). Tackling toddler obesity through a pilot community-based family intervention. Community Practioner, 81(1), 28-31.

Yao, N. L., \& Hillemeier, M. M. (2012). Weight status in Chinese children: maternal perceptions and child selfassessments. World Journal of Pediatrics, 8(2), 129-135.

Young, K. M., Northern, J. J., Lister, K. M., Drummond, J. A., \& O'Brien, W. H. (2007). A meta-analysis of family-behavioral weight-loss treatments for children. Clinical Psychology Review, 27(2), 240-249.

Zeller, M. H., Boles, R. E., \& Reiter- Purtill, J. (2008). The additive and interactive effects of parenting style and temperament in obese youth seeking treatment. International Journal of Obesity, 32(10), 14741480.

Zeller, M. H., Reiter- Purtill, J., Modi, A. C., Gutzwiller, J., Vannatta, K., \& Davies, W. H. (2007). Controlled study of critical parent and family factors in the obesigenic environment. Obesity, 15(1), 126-136. 

| 211

Summary

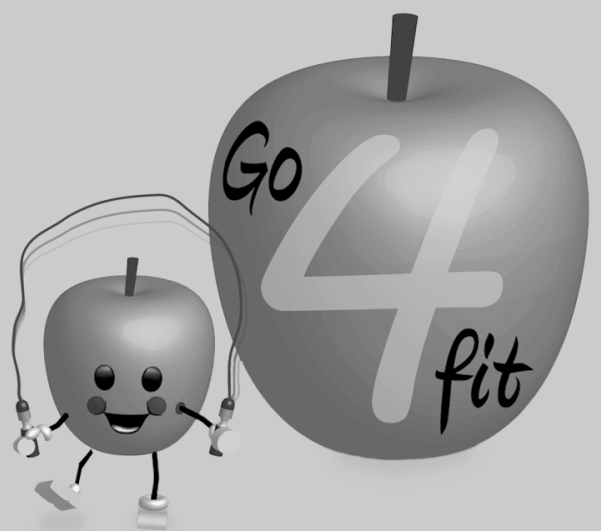


The prevalence of childhood overweight and obesity has dramatically increased worldwide. In the Netherlands, $12.8 \%$ of boys and $14.8 \%$ of girls, aged 2 to 21 years, can be classified as overweight and $1.8 \%$ of boys and $2.2 \%$ of the girls as obese. Children who are overweight are at increased risk for becoming our obese generation and at increased risk for developing physical and psychosocial disorders. It is important to start prevention of childhood obesity and overweight at a young age. Lifestyle Triple $P$ is based on the Positive Parenting Program (Triple P). Triple P was developed by the University of Queensland in Australia and consists of 5 different levels of interventions with increasing intensity: from level 1 (mass media campaigns) to level 5 (parenting support in combination with addressing additional risk factors during the intervention). Lifestyle Triple $P$ is a level 5 intervention, which aims to prevent excessive weight gain in overweight and obese children and consists of 14 sessions: 10 group sessions for parents and 4 individual telephone sessions. Only one previous trial has been done to assess the effects of Lifestyle Triple P: a randomized controlled trial (RCT) in Australia showed positive intervention effects on children's body composition. In order to investigate whether the Lifestyle Triple P intervention works in the Netherlands as well, the intervention was translated and adapted to the Dutch context. The aim of the current thesis is to describe the rationale, implementation, and evaluation of the Lifestyle Triple P intervention in the Netherlands, and reports on seven studies.

After a general introduction in chapter 1 , the results of a systematic literature review are described in chapter 2. A distinction is made between general parenting and specific parenting practices that parents use in raising their children. General parenting is the emotional climate determined by parents' attitudes and beliefs, for example how strict parents are. Parenting practices are specific parenting behaviors regarding topics like food and/or physical activity. The aim of the study was to summarize the findings of observational research regarding the influence of general parenting on children's diet and activity behaviors and weight status. Thirty-six studies were included in the review. Overall, results indicate that children raised in authoritative homes (both responsive and demanding), were found to eat more healthily, be more physically active and have lower BMI scores than children raised with a different style. Differences between studies were due to differences in the conceptualization of parenting constructs. Moderation studies showed that parenting can have differential effects on children's weight-related outcome measures, depending on the characteristics of the child and the parents.

Chapter 3 elaborates on the subject of general parenting. This literature review provides an overview of intervention studies targeting at general parenting in order to prevent or treat childhood obesity. Seven studies could be included in the review. These studies were based on four different interventions to improve general parenting, such as the book 'Living with Children' by Patterson and the 'Triple P intervention' 
by Sanders. All studies showed small to moderate effects on at least one outcome measure (nutrition, physical activity or weight status). The studies showed that general parenting is modifiable. More intervention studies need to be developed to improve general parenting in order to prevent or manage childhood obesity.

Chapter 4 reports on an observational study, in which data of the KOALA study (a birth cohort study in the Netherlands) were analyzed. Three research questions were addressed. (1) Are parents able to determine their child's weight status at age 5 years (in terms of underweight, normal weight or overweight)? (2) Which factors predict whether parents correctly perceive their child's weight status? (3) What is the predictive value of accurate weight status perception in explaining children's longitudinal weight development? Parents of 2066 5-year-old children returned questionnaires, in which they were asked to report their children's weight status and actual height and weight. Eighty-five percent op the parents of the overweight children underestimated their child's weight status. Predictors of accurate weight status perception were children's actual BMI z-scores at ages 2 and 5 years. Furthermore, children whose parents correctly perceived their child as overweight had a higher BMI over time compared to children whose parents underestimated their weight. Parental awareness of their child's overweight as such is thus not sufficient for subsequent effective weight management by parents.

Chapter 5 reports on a validation study of the Lifestyle Behavior Checklist (LBC). The LBC consists of 2 subscales: the Problem scale, measuring the extent to which parents perceive overweight-related problem behavior in their children, and the Confidence scale, which measures the extent to which parents have enough self-efficacy to manage their children's problem behavior. The LBC was developed by researchers of the University of Queensland in Australia. It was systematically translated into Dutch and distributed via an online survey panel $(\mathrm{N}=392)$. Its construct validity was determined using three general parenting scales: restrictiveness, nurturance, and psychological control. Parents were asked to fill out the questionnaire again after two weeks, in order to determine test-retest reliability. The Dutch translation of the LBC was found to be a reliable and reasonably valid questionnaire to measure parental perceptions of children's weight-related problem behavior and the extent to which parents feel confident about managing these problems.

Chapter 6 presents the results of semi-structured interviews with Dutch youth health care (YHC) professionals (physicians, nurses, and management staff). The interviews were set up as a result of the disappointing number of referrals of parents of overweight children to our pilot intervention. One of the tasks of the YHC is to systematically monitor children's weight status, and YHC professionals were asked to refer parents of overweight children to an overweight prevention intervention. The research model for the interviews was based on the Implementation Theory by Fleuren. We 
distinguished five different categories of factors determining the implementation rate of interventions: (1) characteristics of the socio-political environment or the context, (2) characteristics of the organization, (3) characteristics of the adopting person (user), (4) characteristics of the innovation, and (5) characteristics of the participants. According to the YHC professionals, the most important barriers to referring parents of overweight children were related to characteristics of the parents (resistance towards discussing weight issues, denial of the overweight problem) and their own characteristics (insufficient skills to discuss overweight problems with parents and low self-efficacy). It is important that parents become more aware of their child's overweight and that YHC professionals develop more confidence in their skills for motivating parents of overweight children to participate in obesity prevention interventions.

The protocol of our randomized controlled trial is outlined in chapter 7 . The primary goal of the study was to assess the effects of the Lifestyle Triple P intervention on anthropometric outcome measures (BMI z-scores, waist circumference, and skinfold thickness). In addition, effects of the intervention were assessed on child behavior (diet and physical activity level) and parenting behavior (parenting practices, feeding styles, parenting styles, and self-efficacy). After the baseline measurements, parents were randomly assigned to the either the intervention or the control condition. Parents in the intervention condition received the 14-week Lifestyle Triple P intervention, in which they learn a range of strategies relating to nutrition, physical activity, and positive parenting. Parents in the control condition received two brochures (on diet, physical activity and positive parenting), web-based tailored advice and suggestions for exercises to increase active playing at home. Measurements were conducted immediately after the intervention ( 4 months after baseline) and in the longer term (one year after baseline).

Chapter 8 reports on the effectiveness of the Lifestyle Triple $P$ intervention. The intervention had no effect on the primary outcome measure (BMI z-score, waist circumference, and skinfolds). Analyses of secondary outcomes showed intervention effects on child behavior and on parenting behavior. Improved child behavior outcomes were soft-drink consumption (short-term), sedentary behavior (long-term), and playing outside (long-term). Furthermore, the following parenting behaviors improved: responsibility regarding physical activity (short-term), monitoring food intake (longterm), responsibility regarding nutrition (long-term), encouragement to eat (shortterm), psychological control (short-term), and satisfaction and efficacy in parenting (short-term). Based on the current study, adjustments are recommended for the content of the intervention.

The final chapter of the thesis, chapter 9, summarizes the main findings of the different studies. It also presents methodological considerations concerning the recruitment of study participants, the study population, the broader context of parent- 
ing, the intervention content, implementation of the intervention, outcome measures and statistical analyses. In addition, implications for practice, theory, and further research are described. The main conclusion of this thesis is that although the Lifestyle Triple $P$ intervention showed positive effects on various child behaviors and parenting measures, no effects were found on children's body composition. 



\section{Samenvatting}

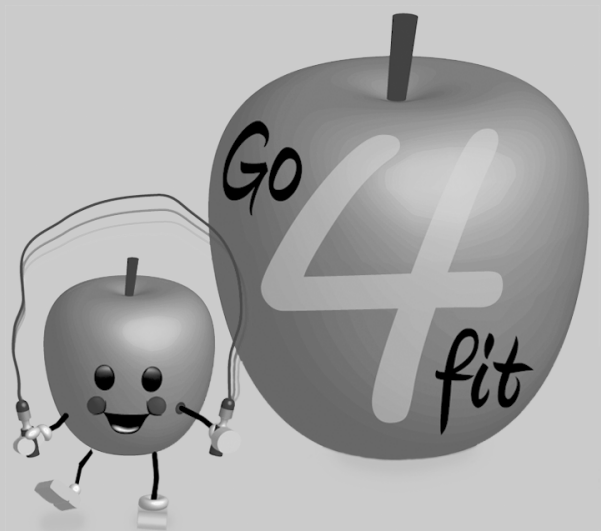


De prevalentie van overgewicht en obesitas bij kinderen is de laatste jaren sterk toegenomen. In Nederland hebben $12.8 \%$ van de jongens en $14.8 \%$ van de meisjes overgewicht, in de leeftijd van 2 tot 21 jaar en $1.8 \%$ van de jongens en $2.2 \%$ van de meisjes zelfs obesitas. Kinderen die overgewicht hebben worden vaak de volwassen generatie met obesitas en bovendien hebben deze kinderen vaak te maken met fysieke en psychosociale problemen. Het is daarom belangrijk om op jonge leeftijd te starten met preventie van overgewicht. Lifestyle Triple $P$ is gebaseerd op het uit Australie afkomstige Positief Pedagogisch Programma (Triple P). Triple $\mathrm{P}$ is ontwikkeld door de University of Queensland en bestaat uit vijf verschillende niveaus van interventies in toenemende intensiteit. Lifestyle Triple $P$ is een niveau 5 -interventie gericht op preventie van excessieve gewichtstoename bij kinderen met overgewicht en obesitas en bestaat uit 14 sessies: tien groepssessies en vier individuele telefonische sessies voor ouders. Een gerandomiseerde studie in Australië liet positieve interventie effecten zien op BMI van de kinderen. Dit is slechts de enige evaluatiestudie naar Lifestyle Triple $P$ en voordat een interventie landelijk geïmplementeerd wordt is het goed eerst te onderzoeken of de interventie in Nederland ook positieve effecten oplevert. Het doel van dit proefschrift is om de rationale, implementatie en evaluatie te beschrijven van de Lifestyle Triple $P$ interventie in Nederland aan de hand van 7 verschillende studies.

$\mathrm{Na}$ het beschrijven van de algemene inleiding in hoofdstuk 1, worden in hoofdstuk 2 de resultaten van een systematische literatuurstudie beschreven. Er wordt hierbij een onderscheid gemaakt tussen algemene opvoeding en specifieke opvoedpraktijken die ouders gebruiken bij het opvoeden van hun kinderen. Algemene opvoeding is het emotionele klimaat dat ouders thuis hanteren, bijvoorbeeld de mate waarin ouders strikt zijn. Opvoedpraktijken daarentegen zijn de concrete gedragingen die ouders uitoefenen met betrekking tot bijvoorbeeld voeding en beweging. Het doel was om te bekijken wat eerdere observationele studies rapporteren betreffende de invloed van algemene opvoeding op voeding, beweging en gewichtsstatus van kinderen. Zesendertig studies konden worden geïncludeerd. Deze studies lieten over het algemeen zien dat kinderen die op een autoritatieve manier zijn opgevoed (d.w.z. ouders die zowel strikt, als betrokken zijn) gezonder eten, meer fysiek actief zijn en een lagere BMI status hebben dan kinderen die op een andere manier (bijv. autoritair) zijn opgevoed. Verschillen tussen studies zijn te wijten aan verschillen in conceptualisatie van opvoedingsconstructen. In moderatiestudies bleek het effect van algemene opvoeding op gewicht-gerelateerde uitkomsten bij kinderen afhankelijk van de kenmerken van de kinderen en van de ouders (bijv. persoonlijkheid).

Hoofdstuk 3 gaat verder in op algemene opvoeding. In deze literatuurstudie is onderzocht in hoeverre er interventiestudies zijn die zich richten op het verbeteren van de algemene opvoeding, met als doel om overgewicht en obesitas bij kinderen te voorkomen of te behandelen. Zeven studies konden worden geïncludeerd. Deze stu- 
dies maakten gebruik van 4 verschillende interventies om algemene opvoeding te verbeteren, bijvoorbeeld het boek 'Living with children' van Patterson, en de interventie 'Triple $\mathrm{P}^{\prime}$ 'van Sanders. Alle studies lieten kleine tot gemiddelde effecten zijn op minstens één uitkomstmaat (voeding, beweging of gewicht). De studies laten zien dat algemene opvoeding veranderbaar is. Het is noodzakelijk dat meer interventiestudies zich gaan richten op het verbeteren van de algemene opvoeding, gezien het belang van deze factor in het ontstaan van overgewicht.

Hoofdstuk 4 is een observationele studie. Hiervoor zijn data van de KOALA studie gebruikt, een geboortecohort in Nederland. De volgende onderzoeksvragen stonden centraal: (1) in hoeverre zijn ouders in staat de gewichtsstatus (ondergewicht, normaal gewicht, overgewicht) van hun 5-jarige kinderen in te schatten?, (2) welke factoren voorspellen of ouders de gewichtsstatus goed inschatten? en (3) wat is de daadwerkelijk longitudinale gewichtsontwikkeling van de kinderen (tot en met 9 jaar) waarvan hun ouders de gewichtsstatus goed schatten dan wel verkeerd schatten? De ouders vulden op 5-jarige leeftijd van hun kinderen 2066 vragenlijsten in, waarin bijvoorbeeld gevraagd werd naar de gewichtsstatus en het gewicht en de lengte van het kind. Van de kinderen die overgewicht hadden, onderschatte $85 \%$ van de ouders de gewichtsstatus van het kind. Voorspellers van het correct schatten waren de daadwerkelijke BMI zscores op 2 en 5 jaar. Daarnaast hadden de kinderen waarvan de ouders hun gewichtsstatus goed schatten over de tijd een hogere BMI. Kinderen met een hogere BMI hebben dus een grotere kans dat hun ouders zien dat ze te zwaar zijn, maar bewustzijn voor ouders alleen is niet genoeg om dit overgewicht op te lossen.

Hoofdstuk 5 betreft een validatiestudie van een vragenlijst (Lifestyle Behavior Checklist; LBC) die bestaat uit 2 subschalen. De eerste schaal (problemscale) meet de mate waarin ouders probleemgedrag ervaren bij hun kinderen, gerelateerd aan overgewicht. De tweede schaal (confidencescale) meet in hoeverre ouders vertrouwen hebben om met dit probleemgedrag om te gaan. Deze vragenlijst is oorspronkelijk ontwikkeld door onderzoekers van de University of Queensland in Australië. Voor de huidige studie is de lijst systematisch vertaald in het Nederlands en is deze vervolgens bij 392 ouders afgenomen via een online panel. Om de constructvaliditeit van de vragenlijst te bepalen zijn drie algemene opvoedingsschalen gemeten: striktheid, zorgzaamheid en psychologische controle. Daarnaast is de test-hertest betrouwbaarheid van de lijst bepaald door de ouders 2 weken later nog eens dezelfde lijst te laten invullen. De Nederlandse vertaling van de LBC bleek betrouwbaar en redelijk valide om enerzijds het probleemgedrag van kinderen te meten met betrekking tot overgewicht en anderzijds te meten in hoeverre ouders het gevoel hebben dat ze met dit probleemgedrag om kunnen gaan. 
In hoofdstuk 6 worden de resultaten beschreven van interviews met Jeugdgezondheidszorg (JGZ) professionals (artsen, verpleegkundigen en stafleden). De aanleiding voor deze interviews was het tegenvallende aantal doorverwijzingen van ouders van kinderen met overgewicht naar de pilot interventie. Een van de taken van de JGZ is het structureel monitoren van de gewichtsstatus van de kinderen. Kinderen met overgewicht zouden worden doorverwezen naar een interventie ter preventie van obesitas. De implementatietheorie van Fleuren werd gebruikt als uitgangspunt tijdens de interviews. Er zijn 5 verschillende soorten barrières van JGZ professionals te onderscheiden: (1) kenmerken van de sociaal-politieke omgeving, (2) kenmerken van de organisatie, (3) kenmerken van de gebruikers, (4) kenmerken van de innovatie en (5) kenmerken van de deelnemers. JGZ professionals noemden vooral kenmerken van de ouders (weerstand om overgewicht te bespreken, het overgewicht niet willen zien) en kenmerken van zichzelf (over te weinig vaardigheden beschikken om overgewicht bij ouders bespreekbaar te maken en te weinig zelfvertrouwen hebben). Het is belangrijk dat ouders zich bewuster worden van het overgewicht van hun kind en dat JGZ professionals meer vaardigheden en vertrouwen krijgen om overgewicht van het kind met ouders te bespreken.

Het studieprotocol van de gerandomiseerde studie wordt beschreven in hoofdstuk 7. Het primaire doel van de studie was om het effect te bepalen van de Lifestyle Triple $\mathrm{P}$ interventie op antropometrie uitkomstmaten (BMI z-scores, middelomtrek en huidplooien) van het kind. Daarnaast werd onderzocht of de interventie effect had op leefstijlgedragingen van het kind (voeding en beweging) en opvoedgedrag van ouders (ouderschapspraktijken, voedingsstijlen, opvoedstijlen, eigen effectiviteit en voldoening in opvoeden). Ouders die deelnamen aan het onderzoek werden na de baselinemetingen ad random verdeeld over de interventieconditie en de controle conditie. Deelnemers in de interventieconditie ontvingen de 14-weekse Lifestyle Triple P interventie, waarin een groot aantal strategieën behandeld werden met drie centrale onderwerpen: voeding, beweging en positief opvoeden. Deelnemers in de controleconditie ontvingen een brochure over voeding en beweging, een brochure over opvoeden en advies op maat via internet. Om te kunnen bepalen of de interventie effect had vonden uitkomstmetingen plaats direct na de interventie ( 4 maanden na baseline) en op lange termijn (12 maanden na baseline).

Hoofdstuk 8 geeft de resultaten van de Lifestyle Triple $P$ interventie weer. De interventie bleek niet effectief te zijn met betrekking tot de primaire uitkomstmaten (BMI z-score, middelomtrek en huidplooien). Wel werden er effecten gevonden op gedrag van kinderen en opvoedgedrag van ouders. Met betrekking tot gedrag van kinderen werden effecten gevonden op frisdrankconsumptie (korte termijn), tv-kijken en computeren (lange termijn) en buitenspelen (lange termijn). Daarnaast werden effecten gevonden op opvoedgedrag van ouders: verantwoordelijkheid ten aanzien 
van fysieke activiteit (korte termijn), verantwoordelijkheid ten aanzien van voeding (lange termijn), monitoren van de voeding (lange termijn), aanmoedigen om te eten (korte termijn), psychologische controle (korte termijn) en effectiviteit en voldoening van ouders in het opvoeden (korte termijn). Op basis van de resultaten van dit onderzoek zijn aanbevelingen gedaan met betrekking tot aanpassing van de interventie.

In het laatste hoofdstuk van het proefschrift, hoofdstuk 9, worden de belangrijkste bevinden van de verschillende studies besproken. Daarnaast worden methodologische beschouwingen beschreven met betrekking tot de werving, studiepopulatie, het opvoedingsperspectief, de inhoud en implementatie van de interventie, de uitkomstmaten en de statistische analyses. Ten slotte worden implicaties voor de praktijk, theorie en toekomstig onderzoek beschreven. De algemene conclusie van dit proefschrift is dat ondanks dat de Lifestyle Triple P interventie positieve effecten liet zien op gedrag van kinderen en opvoedgedrag van ouders, er geen effecten gevonden werden op de lichaamssamenstelling van kinderen. 



\section{Dankwoord}

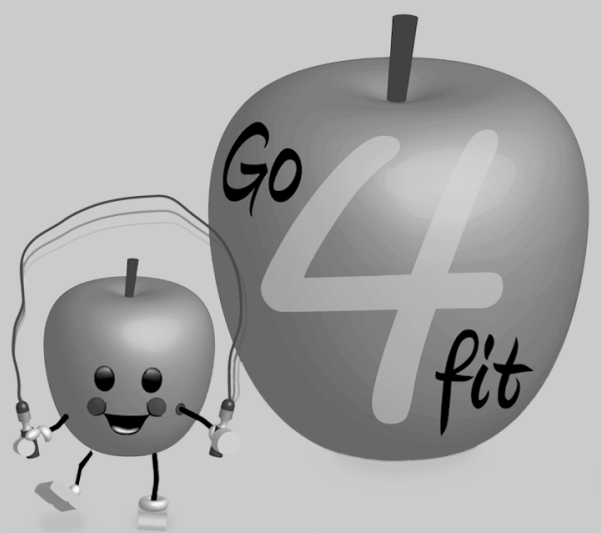


Ook al lijkt promoveren soms een eenzaam proces, zonder hulp van anderen is het een onbegonnen traject. Graag wil ik dan ook iedereen bedanken die op een of andere wijze heeft bijgedragen aan het tot stand komen van dit proefschrift.

Allereerst wil ik mijn drie Promotoren (oftewel Triple P) bedanken: Stef, Pieter en Nanne. Stef, een betere dagelijkse begeleider had ik mij niet kunnen wensen. Vanaf het begin heb jij vertrouwen in mij gehad en heb je me altijd weten te stimuleren mijn grenzen te verleggen. Pieter, bedankt voor je kritische blik. Ik heb van jou geleerd hoe belangrijk het is om nauwkeurig en consistent te werken. En als laatste Nanne, ook jij stond altijd voor me klaar als het nodig was en leerde me vooral alles in een breder perspectief te plaatsen.

Verder wil ik graag de leescommissie, Gerjo Kok, Anita Jansen, Anita Vreugdenhil en Ireen de Graaf, bedanken voor jullie tijd om mijn proefschrift te beoordelen. Nanna Lien, thank you for your time in reading and assessing my thesis.

Daarnaast een woord van dank aan alle assistenten die aan mijn project meegewerkt hebben: Aniek, Leonieke en Femke. Femke, jij hebt me heel erg goed geholpen tijdens de uitvoering van het onderzoek zelf. Ik denk dat wij elkaar goed aanvulden en ik kan nog steeds veel leren van jouw eeuwige geduld. Bedankt dat je mijn paranimf bent!

Collega's van de vakgroep Gezondheidsbevordering: natuurlijk wil ik ook jullie allemaal bedanken en een paar mensen in het bijzonder. Leon en Marja, bij jullie kon ik altijd terecht met al mijn vragen, bedankt voor jullie ondersteuning. Jessica, Ester en Karin, met jullie heb ik alle drie op een prettige manier samengewerkt aan publicaties. Ik hoop dat we dat in de toekomst blijven doen.

Jacky en Antoinette, jullie hebben de groepssessies verzorgd. Bedankt voor de manier waarop jullie dit gedaan hebben. Ik weet zeker dat er geen enthousiastere verpleegkundigen bestaan die zo'n goed team vormen. Marianne, jij bedankt voor alle gezellige momenten tijdens de metingen. Wat hebben we lang zitten wachten op de gezinnen, tot en met de avonduren en de weekenden. Achteraf konden we er gelukkig toch om lachen! Ik wil de GGD bedanken dat we de groepssessies en metingen op hun locaties mochten doen. Daarnaast wil ik alle medewerkers van de jeugdgezondheidszorg bedanken voor het doorverwijzen van geschikte deelnemers en dat ik jullie mocht interviewen. Deelnemers aan het werkgroepoverleg GO4fit van de Academische Werkplaats, bedankt voor de inspirerende bijeenkomsten en jullie input. Maria Jansen en Lidy van der Goot, bedankt voor het scheppen van de juiste voorwaarden om het project tot een goed einde te brengen. Jacqueline van Rijn en Marion van Bommel van het 
Nederlands Jeugd Instituut, bedankt voor de prettige samenwerking. Prof. Matt Sanders, thanks for sharing your experiences in working with Triple P and giving good suggestions for improving our study.

Ook wil ik graag alle deelnemers aan de GO4fit studie, dus zowel de ouders als de kinderen, bedanken voor hun deelname. Zonder jullie had er geen onderzoek kunnen plaatsvinden!

Lieve vrienden en familie, het is jullie niet ontgaan dat een proefschrift schrijven nogal veel tijd kost. Bedankt voor jullie begrip. Jill, bedankt voor alle gezellige en ontspannende momenten tijdens mijn promotietijd! Lieke, wat hebben we een leuke tijd gehad als collega's en als vriendinnen op het Deb.

Papa en mama, bedankt voor jullie gezonde opvoeding; hoe meer ik weet over opvoeding, hoe meer ik me realiseer hoe ontzettend goed jullie je taak hebben volbracht. Ik ben jullie erg dankbaar dat jullie ons geleerd hebben te streven naar het hoogst haalbare, maar ook dat er zoveel meer is in het leven dan werken. Marieke, lief zusje, ik ben heel blij dat jij als paranimf naast me staat vandaag.

Als laatste nog één collega die ik graag wil bedanken en dat ben jij, Dave! Wat hebben we toch een geluk dat we elkaar hebben leren kennen. Bedankt voor al je geduld, je ondersteuning en relativeringsvermogen. Ook al lukt het niet altijd om werk en privé te scheiden, we hebben het toch maar goed samen! I love you! 



\section{Curriculum Vitae}

Sandrine Maria Paulien Leonie Gerards was born on September $1^{\text {st }} 1986$ in Heerlen, the Netherlands. After completing her Atheneum degree at the secondary school in 2004 (Graaf Huyn College, Geleen), she started the Bachelor studies of Health Sciences at Maastricht University. Her specializations were Health Education and Promotion and Movement Sciences. In addition to her studies, she participated in the Honours Programme of Health Sciences. She was an exchange student for four months at the Faculty of Sports and Health Sciences, at the University of Yväskylä in Finland. Her internship took place at the Public Health Service of Amsterdam. After her graduation in 2007, she studied to become a Master of Public Health, with a specialization in Health Education and Promotion. She graduated in 2008. Sanne started to work as a research assistant at the Department of Health Promotion on the September $1^{\text {st }}, 2008$. From December 2008 she worked as a PhD student. She was involved in several teaching related activities and obtained her University Teaching Qualification (BasisKwalificatie Onderwijs). She was trained as a Triple P trainer by Triple P International in November 2012. Since December 2013 she has been working as a postdoctoral researcher at the Department of Health Promotion. 


\section{Publication list}

Gerards, S.M.P.L., Hummel, K., Dagnelie, P.C., De Vries, N.K., Kremers, S.P.J. Parental self-efficacy in childhood overweight: validation of the Lifestyle Behaviour Checklist in the Netherlands. (2013). International Journal of Behavioural Nutrition and Physical Activity. 10 (1): 7.

Kremers, S., Sleddens, E., Gerards, S., Gubbels, J., Rodenburg, G., Gevers, D., Assema, P. General and Food-Specific Parenting: Measures and Interplay. (2013). Childhood Obesity. 9 Suppl 1: S22-31

Gerards, S.M.P.L., Dagnelie, P.C., Jansen, M.W.J., De Vries, N.K., Kremers, S.P.J. Barriers to successful recruitment of parents of overweight children for an obesity prevention intervention: a qualitative study among youth health care professionals. (2012). BMC Family Practice. 13:37

Gerards, S.M.P.L., Dagnelie, P.C., Jansen, M.W.J., Van der Goot, L.O.H.M, De Vries, K., Sanders, M.R., Kremers, S.P.J. Lifestyle Triple P: a parenting intervention for childhood obesity. (2012). BMC Public Health. Apr 3; 12(1): 267

Gerards, S.M.P.L., Sleddens, E.F.C., Dagnelie, P.C., De Vries, N.K., Kremers, S.P.J. (2011). Interventions addressing general parenting to prevent or treat childhood obesity. International Journal of Pediatric Obesity. Jun; 6(2-2): e28-45. 
Sleddens, E.F.C., Gerards, S.M.P.L., Thijs, C., De Vries, N.K., Kremers, S.P.J. General parenting, childhood overweight and obesity-inducing behaviors: a review. (2011). International Journal of Pediatric Obesity. Jun; 6(2-2): e12-27.

Kremers, S., Reubsaet, A., Martens, M., Gerards, S., Jonkers, R., Candel, M., De Weerdt, I., De Vries, N. (2010). Systematic prevention of overweight and obesity in adults: A qualitative and quantitative literature analysis. Obesity Reviews. May; 11(5): 371-9. 
